Estimação de modelos de duração condicional estocástica por meio da função característica empírica

\author{
José Euclides de Melo Ferraz
}

TESE APRESENTADA

$\mathrm{AO}$

Instituto de Matemática e Estatística

DA

Universidade De SÃo PaUlo

PARA

OBTENÇÃO DO TÍTULO

$\mathrm{DE}$

Doutor EM CiênCIAS

Área de Concentração: Estatística

Orientador: Prof. Dr. Pedro Alberto Morettin 



\title{
Estimação de modelos de duração condicional estocástica por meio da função característica empírica
}

\author{
Este exemplar corresponde à redação \\ final da tese devidamente corrigida \\ e defendida por José Euclides de Melo Ferraz \\ e aprovada pela Comissão Julgadora.
}

Banca Examinadora:

- Prof. Dr. Pedro Alberto Morettin (orientador) - IME-USP.

- Profa. Dra. Clélia Maria de Castro Toloi - IME-USP.

- Prof. Dr. Pedro Luiz Valls Pereira - IBMEC-SP.

- Prof. Dr. Cristiano Fernandes - PUC-RJ.

- Prof. Dr. Gauss Moutinho Cordeiro - UFRPE. 



\section{Resumo}

Neste trabalho propomos a utilização do método da função característica empírica (ECF), para estimar os parâmetros do modelo de duração condicional estocástica (SCD). Para determinação das variáveis latentes do processo utilizamos três alternativas: um filtro de Kalman, um filtro obtido por integração numérica e um filtro baseado na expansão de Gram-Charlier até quarta ordem. Os resultados são então aplicados em séries de duração da GE, Microsoft e USD/EUR. 



\begin{abstract}
We propose the use of the Empirical Characteristic Function (ECF) method to estimate the parameters of the Stochastic Conditional Duration (SCD) model. In order to estimate the latent variables we propose the use of three alternatives: a Kalman filter, a filter based on numerical integration (quadrature) and a filter based on the 4th-order Gram-Charlier expansion. The results are applied to the estimation of the parameters of the duration process for GE, Microsoft and USD/EUR.
\end{abstract}



Dedico esta tese a Vitorina, ao Thiago e ao Gabriel, e deixo para vocês um poema do Fernando Pessoa:

Ó mar salgado, quanto do teu sal

São lágrimas de Portugal!

Por te cruzarmos, quantas mães choraram,

Quantos filhos em vão rezaram!

Quantas noivas ficaram por casar

Para que fosses nosso, ó mar!

Valeu a pena? Tudo vale a pena

Se a alma não é pequena.

Quem quer passar além do Bojador

Tem que passar além da dor.

Deus ao mar o perigo e o abismo deu,

Mas nele é que espelhou o céu.

Fernando Pessoa 



\section{Agradecimentos}

Esta tese é resultado da ajuda de muitas pessoas, a quem eu gostaria de manifestar o meu profundo reconhecimento, pois sem elas eu não teria conseguido terminá-la.

Em primeiro lugar, gostaria de agradecer a minha muito amada e querida esposa Vitorina e aos meus dois amados e queridos filhos, Thiago e Gabriel, pelo suporte, carinho e companheirismo, e principalmente pela compreensão do tempo perdido em que simplesmente não pudemos estar juntos.

Gostaria também de agradecer ao professor Morettin pelo constante apoio e inspiração, desde a primeira vez que vim a ele me aconselhar sobre meu interesse em iniciar um doutorado, passando pelas matérias de séries de tempo e análise espectral, que despertaram meu interesse pelo assunto, até agora, no final do trabalho, pela sua atenção aos detalhes e pela sua infinita paciência com meus longos desaparecimentos. Também devo ao professor Morettin a sugestão de trabalhar com modelos de duração condicional estocástica.

A todos os professores do departamento de Estatística do IME-USP deixo meu agradecimento por suas aulas muitas vezes inspiradoras, e que sempre abriam nossa visão sobre algum tema importante da Estatística.

Finalmente, gostaria de agradecer aos meus colegas de Banco Itaú, Christian, Erlei e Lola, que muitas vezes tiveram que cobrir as inúmeras horas que estive ausente cursando as aulas ou simplesmente buscando livros na biblioteca. Também gostaria de agradecer ao Carlos Henrique Mussolini e ao Fábio Oliveira, por permitirem que eu fizesse o doutorado e até mesmo o colocasse como meta de desenvolvimento pessoal.

A todos vocês, muito obrigado! 



\section{Sumário}

Lista de abreviaturas $\quad$ xxv

1 Introdução 1

2 A Função Característica Empírica

2.1 A Função Característica $(\mathrm{CF}) \ldots \ldots \ldots \ldots \ldots \ldots \ldots$

2.1.1 Algumas propriedades da $\mathrm{CF} \ldots \ldots \ldots \ldots$

2.2 A Função Característica Empírica (ECF) . . . . . . . . . . . . . . 7

2.3 Propriedades da ECF . . . . . . . . . . . . . . 8

2.3.1 Séries de v.a. independentes e identicamente distribuidas (i.i.d.) . . . 8

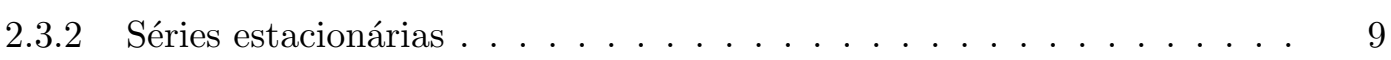

2.4 Sumário . . . . . . . . . . . . . . . . . . . . 12

3 Métodos de Estimação utilizando a ECF 13

3.1 O método da ECF contínua $(\mathrm{CECF}) \ldots \ldots \ldots \ldots$. . . . . . . . . . 14

3.2 O método da ECF discreta $(\mathrm{DECF}) \ldots \ldots \ldots \ldots \ldots$

3.3 Extensão do GMM para um contínuo de momentos . . . . . . . . . . . 19

3.4 Sumário . . . . . . . . . . . . . . . . . . . . . . . 2 22

4 O modelo de Duração Condicional Estocástica (SCD) 23

4.1 O modelo de duração condicional autorregressiva (ACD) . . . . . . . . . 2 24

4.2 O modelo de duração condicional estocástica (SCD) . . . . . . . . . . . 25

4.3 Formulação do modelo de interesse . . . . . . . . . . . . . . . . 27

4.4 Funções características para as distribuições dos log-erros $\varepsilon_{j} \ldots \ldots \ldots$. . . . 31

4.5 Cumulantes e momentos das distribuições dos log-erros $\varepsilon_{j} \ldots \ldots \ldots$. . . . . . 33

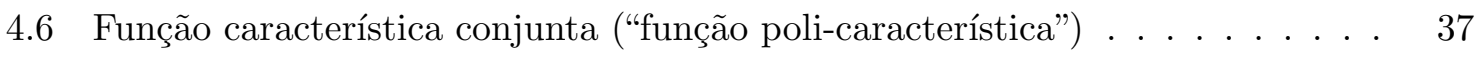

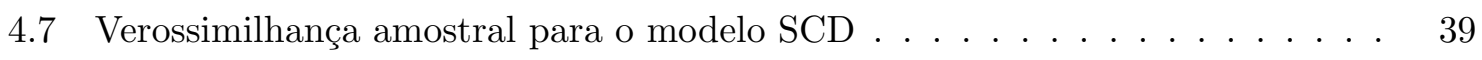

4.8 Sumário . . . . . . . . . . . . . . . . . . . . . . . . . 40

5 Estimação do modelo SCD utilizando a ECF 43

5.1 Estimação dos parâmetros através da CECF . . . . . . . . . . . . . . 43

5.2 Estimação dos parâmetros através da DECF . . . . . . . . . . . . . 50

5.3 Derivadas da função poli-característica em relação ao vetor de parâmetros . . 53 
5.4 Restrições impostas pelo espaço paramétrico . . . . . . . . . . . . . . . . . . . . . . . . .

5.5 Sumário . . . . . . . . . . . . . . . . . . . . . . 55

6 Estimação das variáveis latentes no modelo SCD 57

6.1 Relações recursivas de previsão e filtragem . . . . . . . . . . . . . . 58

6.1.1 Previsão um passo adiante (atualização do tempo) . . . . . . . . . 58

6.1.2 Filtro (atualização após observação) . . . . . . . . . . . . 58

6.1 .3 Suavização . . . . . . . . . . . . . . . . . 59

6.2 Filtro e suavizador de Kalman . . . . . . . . . . . . . . 59

6.2.1 Estimativa por quase-verossimilhança (QML) . . . . . . . . . . . 61

6.3 Procedimento recursivo baseado na expansão de Gram-Charlier ("filtro GramCharlier") ............................. 62

6.3.1 Restrição do espaço paramétrico . . . . . . . . . . . . . . 68

6.4 Sumário . . . . . . . . . . . . . . . . . . . . . . . 70

7 Simulação 71

7.1 Número de nós para quadratura de Gauss-Hermite . . . . . . . . . . . . . . . 75

7.2 Resultados da simulação . . . . . . . . . . . . . . . . . 77

7.2 .1 Desempenho dos estimadores . . . . . . . . . . . . . . . . . . . . . .

7.2 .2 Desempenho dos filtros . . . . . . . . . . . . . 79

7.3 Sumário . . . . . . . . . . . . . . . . . . . . . . . . 90

8 Aplicação empírica 91

8.1 Descrição e tratamento dos dados . . . . . . . . . . . . . . . . . 91

8.2 Duração de volume da GE . . . . . . . . . . . . . . . . . . . 94

8.3 Duração de volume da Microsoft . . . . . . . . . . . . . . . . . . 99

8.4 Sumário . . . . . . . . . . . . . . . . . . . . . . . 104

9 Conclusão 105

A Propriedades de algumas classes de estimadores 107

A.1 Propriedades assintóticas dos estimadores extremos . . . . . . . . . . . . . 107

A.2 O método generalizado dos momentos $(\mathrm{GMM}) \ldots \ldots \ldots \ldots \ldots$

A.3 Um Teorema Central Limite para processos estacionários . . . . . . . . . . 114

B Covariância do processo $Y_{n}$

B.1 Caso i.i.d. . . . . . . . . . . . . . . . . . . . . . 117

B.2 Caso estacionário (não necessariamente i.i.d.) . . . . . . . . . . . 122

B.2.1 Convergência da seqüência $\Omega_{n}(r, s) \ldots \ldots \ldots \ldots$

C Demonstrações dos Capítulos 4 e 5

D Matriz de covariância assintótica do estimador CECF 
E Matriz de covariância assintótica do estimador DECF

F Filtro de Gram-Charlier

G Estimativa das matrizes de covariância dos estimadores CECF e DECF 183

\begin{tabular}{lr} 
H Aplicação empírica - outras estimativas & $\mathbf{1 8 9}$ \\
\hline
\end{tabular}

H.1 Resultados para a GE . . . . . . . . . . . . . . . . . . . . . . . . 189

H.1.1 Duração de negócio . . . . . . . . . . . . . . . . . . . . . . . 189

H.1.2 Duração de preço . . . . . . . . . . . . . . . . . . . . . . . . . 194

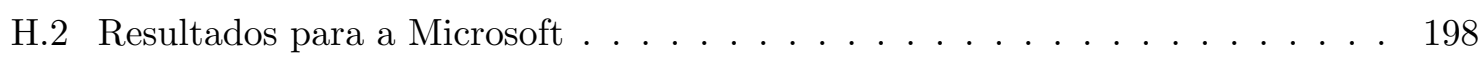

H.2.1 Duração de negócio . . . . . . . . . . . . . . . . . . . . . . 198

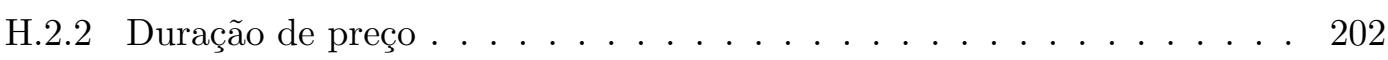

H.3 Resultados para USD/EUR . . . . . . . . . . . . . . . . . . . . . . . . . . . . . . .

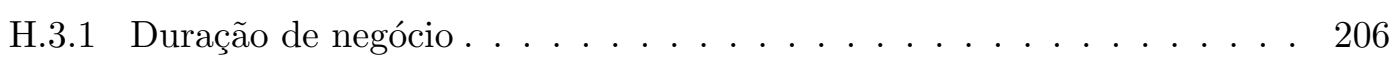

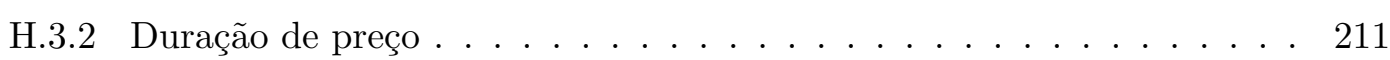

H.3.3 Ln duração de negócio . . . . . . . . . . . . . . . . . . . . . 215

Referências Bibliográficas 



\section{Lista de Figuras}

4.1 Funções densidade de probabilidade da log-exponencial (Gumbel), log-Weibull e log-gama, para $\lambda=0.5 \ldots \ldots \ldots \ldots$

4.2 Funções densidade de probabilidade da log-exponencial (Gumbel), log-Weibull e log-gama, para $\lambda=2$.

4.3 Coeficiente de assimetria $\gamma_{1}$ em função de $\lambda$, para as distribuições log-exponencial, log-Weibull e log-gama.

4.4 Coeficiente de excesso de curtose $\gamma_{2}$ em função de $\lambda$, para as distribuições logexponencial, log-Weibull e log-gama.

5.1 Matriz de covariância $\Omega_{n}(r, s)$ para o processo de duração estocástica condicional, em função do tamanho da amostra $(n)$. Os parâmetros do processo SCD são $\delta=0.033, \phi=0.95, \sigma_{\eta}=0.1$ e $\lambda=2$, com distribuição Weibull. Os pontos para cálculo da função característica (sorteados aleatoriamente) são $r=(0.891299,0.762097)^{\prime}$ e $s=(0.456468,0.018504)^{\prime}$. Para fim de comparação a figura também ilustra a matriz $\Omega_{n}^{i i d}(r, s)$ do processo IID . . . . . . . . . .

6.1 Aproximação de Gram-Charlier para a densidade, sem imposição de restrições (à esquerda) e com imposição de restrições nos parâmetros $\left(\gamma_{1}, \gamma_{2}\right)$ conforme Jondeau e Rockinger (1999) (à direita), para as seguintes distribuições: logexponencial (4.38) em cima, log-Weibull (4.39), $\lambda=0.5$, no meio, e log-gama (4.40), $\lambda=0.5$, em baixo.

6.2 Espaço paramétrico $\left(\gamma_{1}, \gamma_{2}\right)$ para a expansão de Gram-Charlier. A linha cheia indica o "envelope" como indicado pelas equações (6.66) e (6.67), e a região hachurada representa o espaço paramétrico para que a expansão de Gram-Charlier constitua uma densidade de probabilidade.

7.1 Amostras simuladas da duração (à esquerda) e log-duração (à direita) com distribuições condicionais exponencial (cima), Weibull $\lambda=0.5$ (meio) e gama $\lambda=0.5$ (baixo), e parâmetros $\delta=0.033, \phi=0.95, \sigma_{\eta}=0.1$. 
7.2 Partes reais (à esquerda) e imaginárias (à direita) da função característica empírica (ECF), $\operatorname{Re} \varphi_{z_{n}}(r)$, para amostras de tamanho 100 (cima), 1000 (meio) e 10000 (baixo), em função de $r=\left(r_{1}, r_{2}\right)$, com parâmetros $\delta=0.033, \phi=0.95, \sigma_{\eta}=0.1$, $\lambda=1$ (distribuição exponencial), $p=1$, e usando para $r_{\zeta}$ os nós da quadratura de Gauss-Hermite de 39 pontos.

7.3 Partes reais (à esquerda) e imaginárias (à direita) da função característica empírica $(\mathrm{ECF}), \operatorname{Re} \varphi_{z_{n}}(r)$, para amostra de tamanho 100.000 (cima) e para a função característica $(\mathrm{CF}), \operatorname{Re} \varphi_{z}(r)$, (baixo) em função de $r=\left(r_{1}, r_{2}\right)$, com parâmetros $\delta=0.033, \phi=0.95, \sigma_{\eta}=0.1, \lambda=1$ (distribuição exponencial), $p=1$, e usando para $r_{\zeta}$ os nós da quadratura de Gauss-Hermite de 39 pontos.

7.4 Número de nós a uma distância da origem (abscissas) em função do número de nós da quadratura de Gauss-Hermite (ordenadas): na figura de cima a distância máxima é 3 , e na de baixo a distância máxima é $10 \ldots \ldots \ldots$. . . . . . . . .

7.5 Resultado da integral $\int|\varphi(r)| e^{-r^{\prime} r} d r$, em função do número de nós da quadratura de Gauss-Hermite. . . . . . . . . . . . . . . . . . . . . . . .

7.6 Histograma das estimativas do parâmetro $\delta$ obtidas com os métodos QML, CECF e QML em 1000 simulações para a distribuição Weibull de parâmetros $\theta=\left(\delta, \phi, \sigma_{\eta}, \lambda\right)^{\prime}=(0.033,0.95,0.1,0.5)^{\prime} \ldots \ldots \ldots \ldots \ldots$

7.7 Histograma das estimativas do parâmetro $\phi$ obtidas com os métodos QML, CECF e QML em 1000 simulações para a distribuição Weibull de parâmetros $\theta=(0.033,0.95,0.1,0.5)^{\prime}$.

7.8 Histograma das estimativas do parâmetro $\sigma_{\eta}$ obtidas com os métodos QML, CECF e QML em 1000 simulações para a distribuição Weibull de parâmetros $\theta=(0.033,0.95,0.1,0.5)^{\prime}$.

7.9 Histograma das estimativas do parâmetro $\lambda$ obtidas com os métodos QML, CECF e QML em 1000 simulações para a distribuição Weibull de parâmetros $\theta=(0.033,0.95,0.1,0.5)^{\prime}$.

7.10 Variável latente $\psi$ ("psi") da distribuição exponencial com parâmetros $\delta=0.033$, $\phi=0.95, \sigma_{\eta}=0.1, \lambda=1$ (distribuição exponencial), e filtragem obtida através de procedimento de integração numérica ("Bayesian"), através do filtro de Kalman e através do filtro de Gram-Charlier.

A figura de cima é de uma amostra de tamanho 1000 , enquanto que a figura de baixo é uma ampliação dos 100 primeiros dados.

7.11 Variável latente $\psi$ ("psi") da distribuição Weibull com parâmetros $\delta=0.033, \phi=$ $0.95, \sigma_{\eta}=0.1, \lambda=2$, e filtragem obtida através de procedimento de integração numérica ("Bayesian"), através do filtro de Kalman e através do filtro de GramCharlier.

A figura de cima é de uma amostra de tamanho 1000 , enquanto que a figura de baixo é uma ampliação dos 100 primeiros dados. 
7.12 Variável latente $\psi$ ("psi") da distribuição gama com parâmetros $\delta=0.033, \phi=$ 0.95, $\sigma_{\eta}=0.1, \lambda=2$, e filtragem obtida através de procedimento de integração numérica ("Bayesian"), através do filtro de Kalman e através do filtro de GramCharlier.

A figura de cima é de uma amostra de tamanho 1 000, enquanto que a figura de baixo é uma ampliação dos 100 primeiros dados. . . . . . . . . . . . . . . .

7.13 Variável latente $\psi$ ("psi") da distribuição Weibull com parâmetros $\delta=0.033$, $\phi=0.95, \sigma_{\eta}=0.1, \lambda=0.5$, e filtragem obtida através de procedimento de integração numérica ("Bayesian"), através do filtro de Kalman e através do filtro de Gram-Charlier.

A figura de cima é de uma amostra de tamanho 1000 , enquanto que a figura de baixo é uma ampliação dos 100 primeiros dados. . . . . . . . . . . . . .

7.14 Variável latente $\psi$ ("psi") da distribuição gama com parâmetros $\delta=0.033, \phi=$ $0.95, \sigma_{\eta}=0.1, \lambda=0.5$, e filtragem obtida através de procedimento de integração numérica ("Bayesian"), através do filtro de Kalman e através do filtro de GramCharlier.

A figura de cima é de uma amostra de tamanho 1 000, enquanto que a figura de baixo é uma ampliação dos 100 primeiros dados.

8.1 Dados da GE:

Cima: duração de volume (volume duration) e respectiva componente diurna.

Baixo: duração de volume dessazonalizada e respectivo logaritmo.

8.2 Partes real (esquerda) e imaginária (direita) da função característica empírica (cima) e das funções características com distribuição gama para a duração de volume (volume duration) da GE, calculadas em $\theta_{Q M L}$ (meio) e $\theta_{C E C F}$ (baixo), para a malha de 39 nós da quadratura de Gauss-Hermite. . . . . . . . . . . .

8.3 Estimativa da variável latente para a duração de volume (volume duration) da GE.

Cima: de cima para baixo temos os seguinte filtros calculados em $\theta_{C E C F}$ : (1) Log-durações; (2) filtro de Kalman; (3) filtro Bayesiano; (4) filtro Gram-Charlier. Baixo: primeiras 200 observações dos filtros. . . . . . . . . . . . . . .

8.4 De cima para baixo temos os gráficos de quantis dos resíduos obtidos com os filtros calculados em $\theta_{C E C F}$ com distribuição gama para a duração de volume da GE: (1) filtro de Kalman; (2) filtro Bayesiano; (3) filtro Gram-Charlier; (4) função de autocorrelação dos resíduos do filtro de Kalman.

À esquerda vemos os resíduos da equação de observação e à direita os resíduos da equação de estado. As linhas representam os limites do intervalo de $95 \%$ de confiança, obtidos através de simulação. . . . . . . . . . . . . . . .

8.5 Dados da Microsoft:

Cima: duração de volume (volume duration) e respectiva componente diurna.

Baixo: duração de volume dessazonalizada e respectivo logaritmo. 
8.6 Partes real (esquerda) e imaginária (direita) da função característica empírica (cima) e das funções características com distribuição gama para a duração de volume (volume duration) da Microsoft, calculadas em $\theta_{Q M L}$ (meio) e $\theta_{C E C F}$ (baixo), para a malha de 39 nós da quadratura de Gauss-Hermite. . . . . . . . . 101

8.7 Estimativa da variável latente para a duração de volume (volume duration) da Microsoft.

Cima: de cima para baixo temos os seguinte filtros calculados em $\theta_{C E C F}$ : (1) Log-durações; (2) filtro de Kalman; (3) filtro Bayesiano; (4) filtro Gram-Charlier. Baixo: primeiras 200 observações dos filtros. . . . . . . . . . . . . . . . 102

8.8 De cima para baixo temos os gráficos de quantis dos resíduos obtidos com os filtros calculados em $\theta_{C E C F}$ com distribuição gama para a duração de volume da Microsoft: (1) filtro de Kalman; (2) filtro Bayesiano; (3) filtro Gram-Charlier; (4) função de autocorrelação dos resíduos do filtro de Kalman.

À esquerda vemos os resíduos da equação de observação e à direita os resíduos da equação de estado. As linhas representam os limites do intervalo de $95 \%$ de confiança, obtidos através de simulação. . . . . . . . . . . . . . . . .

\section{H.1 Dados da GE:}

Cima: duração de negócio (trade duration) e respectiva componente diurna.

Baixo: duração de negócio dessazonalizada e respectivo logaritmo. . . . . . . . . 190

H.2 Partes real (esquerda) e imaginária (direita) da função característica empírica (cima) e das funções características com distribuição gama para a duração de negócio (trade duration) da GE, calculadas em $\theta_{Q M L}$ (meio) e $\theta_{C E C F}$ (baixo), para a malha de 39 nós da quadratura de Gauss-Hermite. . . . . . . . . . . . . . 191

H.3 Estimativa da variável latente para a duração de negócio (trade duration) da GE. Cima: de cima para baixo temos os seguinte filtros calculados em $\theta_{C E C F}$ : (1) Log-durações; (2) filtro de Kalman; (3) filtro Bayesiano; (4) filtro Gram-Charlier. Baixo: primeiras 200 observações dos filtros. . . . . . . . . . . . . . . . . . 192

H.4 De cima para baixo temos os gráficos de quantis dos resíduos obtidos com os filtros calculados em $\theta_{C E C F}$ com distribuição gama para a duração de negócio da GE: (1) filtro de Kalman; (2) filtro Bayesiano; (3) filtro Gram-Charlier; (4) função de autocorrelação dos resíduos do filtro de Kalman.

À esquerda vemos os resíduos da equação de observação e à direita os resíduos da equação de estado. As linhas representam os limites do intervalo de $95 \%$ de confiança, obtidos através de simulação. . . . . . . . . . . . . . . . . . . 193

H.5 Dados da GE:

Cima: duração de preço (price duration) e respectiva componente diurna.

Baixo: duração de preço dessazonalizada e respectivo logaritmo. 
H.6 Partes real (esquerda) e imaginária (direita) da função característica empírica (cima) e das funções características com distribuição gama para a duração de preço (price duration) da GE, calculadas em $\theta_{Q M L}$ (meio) e $\theta_{C E C F}$ (baixo), para a malha de 39 nós da quadratura de Gauss-Hermite. . . . . . . . . . . . . . . 195

H.7 Estimativa da variável latente para a duração de preço (price duration) da GE. Cima: de cima para baixo temos os seguinte filtros calculados em $\theta_{C E C F}$ : (1) Log-durações; (2) filtro de Kalman; (3) filtro Bayesiano; (4) filtro Gram-Charlier. Baixo: primeiras 200 observações dos filtros. . . . . . . . . . . . . . . . 196

H.8 De cima para baixo temos os gráficos de quantis dos resíduos obtidos com os filtros calculados em $\theta_{C E C F}$ com distribuição gama para a duração de preço da GE: (1) filtro de Kalman; (2) filtro Bayesiano; (3) filtro Gram-Charlier; (4) função de autocorrelação dos resíduos do filtro de Kalman.

À esquerda vemos os resíduos da equação de observação e à direita os resíduos da equação de estado. As linhas representam os limites do intervalo de $95 \%$ de confiança, obtidos através de simulação. . . . . . . . . . . . . . . . .

H.9 Dados da Microsoft:

Cima: duração de negócio (trade duration) e respectiva componente diurna.

Baixo: duração de negócio dessazonalizada e respectivo logaritmo. . . . . . . . . .

H.10 Partes real (esquerda) e imaginária (direita) da função característica empírica (cima) e das funções características com distribuição gama para a duração de negócio (trade duration) da Microsoft, calculadas em $\theta_{Q M L}$ (meio) e $\theta_{C E C F}$ (baixo), para a malha de 39 nós da quadratura de Gauss-Hermite. . . . . . . . . . . . .

H.11 Estimativa da variável latente para a duração de negócio (trade duration) da Microsoft.

Cima: de cima para baixo temos os seguinte filtros calculados em $\theta_{C E C F}$ : (1) Log-durações; (2) filtro de Kalman; (3) filtro Bayesiano; (4) filtro Gram-Charlier. Baixo: primeiras 200 observações dos filtros. . . . . . . . . . . . . . . . . 200

H.12 De cima para baixo temos os gráficos de quantis dos resíduos obtidos com os filtros calculados em $\theta_{C E C F}$ com distribuição gama para a duração de negócio da Microsoft: (1) filtro de Kalman; (2) filtro Bayesiano; (3) filtro Gram-Charlier; (4) função de autocorrelação dos resíduos do filtro de Kalman.

À esquerda vemos os resíduos da equação de observação e à direita os resíduos da equação de estado. As linhas representam os limites do intervalo de $95 \%$ de confiança, obtidos através de simulação. . . . . . . . . . . . . . . . . . . 201

H.13 Dados da Microsoft:

Cima: duração de preço (price duration) e respectiva componente diurna.

Baixo: duração de preço dessazonalizada e respectivo logaritmo. . . . . . . . . . 202

H.14 Partes real (esquerda) e imaginária (direita) da função característica empírica (cima) e das funções características com distribuição gama para a duração de preço (price duration) da Microsoft, calculadas em $\theta_{Q M L}$ (meio) e $\theta_{C E C F}$ (baixo), para a malha de 39 nós da quadratura de Gauss-Hermite. . . . . . . . . . . . . 203 
H.15 Estimativa da variável latente para a duração de preço (price duration) da Microsoft.

Cima: de cima para baixo temos os seguinte filtros calculados em $\theta_{C E C F}$ : (1) Log-durações; (2) filtro de Kalman; (3) filtro Bayesiano; (4) filtro Gram-Charlier. Baixo: primeiras 200 observações dos filtros. . . . . . . . . . . . . . . . . . 204

H.16 De cima para baixo temos os gráficos de quantis dos resíduos obtidos com os filtros calculados em $\theta_{C E C F}$ com distribuição gama para a duração de preço da Microsoft: (1) filtro de Kalman; (2) filtro Bayesiano; (3) filtro Gram-Charlier; (4) função de autocorrelação dos resíduos do filtro de Kalman.

À esquerda vemos os resíduos da equação de observação e à direita os resíduos da equação de estado. As linhas representam os limites do intervalo de $95 \%$ de confiança, obtidos através de simulação. . . . . . . . . . . . .

H.17 Dados de USD/EUR:

Cima: duração de negócio (trade duration) e respectiva componente diurna.

Baixo: duração de negócio dessazonalizada e respectivo logaritmo. . . . . . . . . 206

H.18 Partes real (esquerda) e imaginária (direita) da função característica empírica (cima) e das funções características com distribuição gama para a duração de negócio (trade duration) de USD/EUR, calculadas em $\theta_{Q M L}$ (meio) e $\theta_{C E C F}$ (baixo), para a malha de 39 nós da quadratura de Gauss-Hermite. . . . . . . . . . . . . 208

H.19 Estimativa da variável latente para a duração de negócio (trade duration) de USD/EUR.

Cima: de cima para baixo temos os seguinte filtros calculados em $\theta_{C E C F}$ : (1) Log-durações; (2) filtro de Kalman; (3) filtro Bayesiano; (4) filtro Gram-Charlier. Baixo: primeiras 200 observações dos filtros. . . . . . . . . . . . . . . . 209

H.20 De cima para baixo temos os gráficos de quantis dos resíduos obtidos com os filtros calculados em $\theta_{C E C F}$ com distribuição gama para a duração de negócio de USD/EUR: (1) filtro de Kalman; (2) filtro Bayesiano; (3) filtro Gram-Charlier; (4) função de autocorrelação dos resíduos do filtro de Kalman.

À esquerda vemos os resíduos da equação de observação e à direita os resíduos da equação de estado. As linhas representam os limites do intervalo de $95 \%$ de confiança, obtidos através de simulação. . . . . . . . . . . . . . . . .

H.21 Dados de USD/EUR:

Cima: duração de preço (price duration) e respectiva componente diurna.

Baixo: duração de preço dessazonalizada e respectivo logaritmo. . . . . . . . . . . 211

H.22 Partes real (esquerda) e imaginária (direita) da função característica empírica (cima) e das funções características com distribuição gama para a duração de preço (price duration) de USD/EUR, calculadas em $\theta_{Q M L}$ (meio) e $\theta_{C E C F}$ (baixo), para a malha de 39 nós da quadratura de Gauss-Hermite. . . . . . . . . . . . . . . . . 
H.23 Estimativa da variável latente para a duração de preço (price duration) de USD/EUR.

Cima: de cima para baixo temos os seguinte filtros calculados em $\theta_{C E C F}$ : (1) Log-durações; (2) filtro de Kalman; (3) filtro Bayesiano; (4) filtro Gram-Charlier. Baixo: primeiras 200 observações dos filtros. . . . . . . . . . . . . . . . . 213

H.24 De cima para baixo temos os gráficos de quantis dos resíduos obtidos com os filtros calculados em $\theta_{C E C F}$ com distribuição gama para a duração de preço de USD/EUR: (1) filtro de Kalman; (2) filtro Bayesiano; (3) filtro Gram-Charlier; (4) função de autocorrelação dos resíduos do filtro de Kalman.

À esquerda vemos os resíduos da equação de observação e à direita os resíduos da equação de estado. As linhas representam os limites do intervalo de $95 \%$ de confiança, obtidos através de simulação. . . . . . . . . . . . . . . . . . 214

H.25 Dados de USD/EUR:

Cima: duração de negócio (trade duration) e respectivo logaritmo.

Baixo: componente diurno do logaritmo da duração de negócio e logaritmo da duração de negócio dessazonalizado. . . . . . . . . . . . . . . . . 216

H.26 Partes real (esquerda) e imaginária (direita) da função característica empírica (cima) e das funções características com distribuição gama para a duração de negócio (trade duration) de USD/EUR, calculadas em $\theta_{Q M L}$ (meio) e $\theta_{C E C F}$ (baixo), para a malha de 39 nós da quadratura de Gauss-Hermite. . . . . . . . . . . . . 217

H.27 Estimativa da variável latente para a duração de negócio (trade duration) de USD/EUR.

Cima: de cima para baixo temos os seguinte filtros calculados em $\theta_{C E C F}$ : (1) Log-durações; (2) filtro de Kalman; (3) filtro Bayesiano; (4) filtro Gram-Charlier. Baixo: primeiras 200 observações dos filtros. . . . . . . . . . . . . . . . . 218

H.28 De cima para baixo temos os gráficos de quantis dos resíduos obtidos com os filtros calculados em $\theta_{C E C F}$ com distribuição gama para a duração de negócio de USD/EUR: (1) filtro de Kalman; (2) filtro Bayesiano; (3) filtro Gram-Charlier; (4) função de autocorrelação dos resíduos do filtro de Kalman.

À esquerda vemos os resíduos da equação de observação e à direita os resíduos da equação de estado. As linhas representam os limites do intervalo de $95 \%$ de confiança, obtidos através de simulação. . . . . . . . . . . . . . . . . . 219 



\section{Lista de Tabelas}

7.1 Resumo dos resultados (média, desvio padrão, erro quadrático médio e desvio absoluto médio) das estimativas amostrais dos parâmetros de 1000 simulações usando os algoritmos QML, CECF e DECF, para distribuições exponencial, Weibull e gama com parâmetros $\delta=0.033, \phi=0.95, \sigma_{\eta}=0.1, \lambda$ variável conforme tabela, tamanho de amostra 10000 e ponto inicial $\theta=(0,0.5,0.5,1)^{\prime}$.

$\mathrm{O}$ algoritmo de otimização utilizado foi o fminsearch do Matlab para os métodos QML e CECF e o lsqnonlin para o método DECF, com parâmetros tol $X=10^{-6}$ (tolerância da variável de decisão) e tolFun $=10^{-6}$ (tolerância da função objetivo). Para os algoritmos CECF e DECF foram usados 130 nós da quadratura de Gauss-Hermite, limitados a uma distância 3 da origem. EQM = erro quadrático médio e DAM $=$ desvio absoluto médio. . . . . . . . . . . . . . . .

7.2 Estimativa dos desvios padrão do estimador CECF para o modelo SCD de parâmetros $\delta=0.033, \phi=0.95, \sigma_{\eta}=0.1$, com $\lambda$ e distribuições variáveis conforme a tabela, para amostras de tamanho 10 000. Mais detalhes sobre as estimativas podem ser encontrados no texto e no apêndice G . . . . . . . . . . . . . . . .

7.3 Estimativa dos desvios padrão do estimador DECF para o modelo SCD de parâmetros $\delta=0.033, \phi=0.95, \sigma_{\eta}=0.1$, com $\lambda$ e distribuições variáveis conforme a tabela, para amostras de tamanho 10 000. Mais detalhes sobre as estimativas podem ser encontrados no texto e no apêndice G . . . . . . . . . . . . . . .

7.4 Erro quadrático médio (EQM), desvio absoluto médio (DAM) e mediana dos desvios absolutos (MDA) dos filtros de Kalman, Bayesiano e de Gram-Charlier utilizados para estimação da variável latente, obtidos com simulações de amostras de tamanho 1 000, calculados nos parâmetros $\theta=(0.033,0.95,0.10, \lambda)$ e $\lambda=0.5,1$ (dist. exponencial) e $2 \ldots \ldots \ldots \ldots \ldots$

8.1 Resumo dos dados das amostras de durações dessazonalizadas de GE, Microsoft e USD/EUR. Veja o texto para mais detalhes da base de dados e respectivo trata-

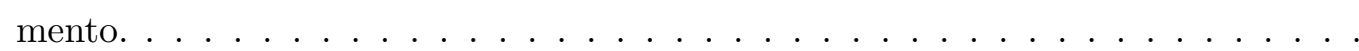

8.2 Estimação dos parâmetros do modelo SCD para a duração de volume (volume duration) da GE, considerando distribuições Weibull e gama para os erros. . . .

8.3 Estimação dos parâmetros do modelo SCD para a duração de volume (volume duration) da Microsoft, considerando distribuições Weibull e gama para os erros. 
G.1 Estimativa da matriz de covariância do modelo SCD com distribuição exponencial de parâmetros $\delta=0.033, \phi=0.95, \sigma_{\eta}=0.1$, através do método CECF. Veja o texto para mais detalhes. . . . . . . . . . . . . . . .

G.2 Estimativa da matriz de covariância do modelo SCD com distribuição Weibull de parâmetros $\delta=0.033, \phi=0.95, \sigma_{\eta}=0.1$ e $\lambda=2$, através do método CECF. Veja o texto para mais detalhes. . . . . . . . . . . . . .

G.3 Estimativa da matriz de covariância do modelo SCD com distribuição gama de parâmetros $\delta=0.033, \phi=0.95, \sigma_{\eta}=0.1$ e $\lambda=2$, através do método CECF. Veja o texto para mais detalhes. . . . . . . . . . . . . . .

G.4 Estimativa da matriz de covariância do modelo SCD com distribuição Weibull de parâmetros $\delta=0.033, \phi=0.95, \sigma_{\eta}=0.1$ e $\lambda=0.5$, através do método CECF. Veja o texto para mais detalhes.

G.5 Estimativa da matriz de covariância do modelo SCD com distribuição gama de parâmetros $\delta=0.033, \phi=0.95, \sigma_{\eta}=0.1$ e $\lambda=0.5$, através do método CECF. Veja o texto para mais detalhes. . . . . . . . . . . .

G.6 Estimativa da matriz de covariância do modelo SCD com distribuição exponencial de parâmetros $\delta=0.033, \phi=0.95, \sigma_{\eta}=0.1$, através do método DECF. Veja o texto para mais detalhes. . . . . . . . . . . . . . .

G.7 Estimativa da matriz de covariância do modelo SCD com distribuição Weibull de parâmetros $\delta=0.033, \phi=0.95, \sigma_{\eta}=0.1$ e $\lambda=2$, através do método DECF. Veja o texto para mais detalhes. . . . . . . . . . . . . . . . .

G.8 Estimativa da matriz de covariância do modelo SCD com distribuição gama de parâmetros $\delta=0.033, \phi=0.95, \sigma_{\eta}=0.1$ e $\lambda=2$, através do método DECF. Veja o texto para mais detalhes. . . . . . . . . . . . . .

G.9 Estimativa da matriz de covariância do modelo SCD com distribuição Weibull de parâmetros $\delta=0.033, \phi=0.95, \sigma_{\eta}=0.1$ e $\lambda=0.5$, através do método DECF. Veja o texto para mais detalhes.

10 Estimativa da matriz de covariância do modelo SCD com distribuição gama de parâmetros $\delta=0.033, \phi=0.95, \sigma_{\eta}=0.1$ e $\lambda=0.5$, através do método CECF. Veja o texto para mais detalhes.

H.1 Estimação dos parâmetros do modelo SCD para a duração de negócio (trade duration) da GE, considerando distribuições Weibull e gama para os erros. . . . . .

H.2 Estimação dos parâmetros do modelo SCD para a duração de preço (price duration) da GE, considerando distribuições Weibull e gama para os erros. . . . . . . .

H.3 Estimação dos parâmetros do modelo SCD para a duração de negócio (trade duration) da Microsoft, considerando distribuições Weibull e gama para os erros. . 198

H.4 Estimação dos parâmetros do modelo SCD para a duração de preço (price duration) da Microsoft, considerando distribuições Weibull e gama para os erros. . . . 202

H.5 Estimação dos parâmetros do modelo SCD para a duração de negócio (trade duration) de USD/EUR, considerando distribuições Weibull e gama para os erros. . 
H.6 Estimação dos parâmetros do modelo SCD para a duração de preço (price duration) de USD/EUR, considerando distribuições Weibull e gama para os erros. . . 211

H.7 Estimação dos parâmetros do modelo SCD para a duração de negócio (trade $d u$ ration) de USD/EUR, considerando distribuições Weibull e gama para os erros. . 216 



\section{Lista de abreviaturas}

ACD duração condicional autoregressiva (Autoregressive Conditional Duration)

a.s. quase certamente (almost surely)

CECF método da ECF contínua (Continuous ECF)

CF função característica (Characteristic Function)

d. em distribuição (in distribution)

DAM desvio absoluto médio

DECF método da ECF discreta (Discrete ECF)

ECF função característica empírica (Empirical Characteristic Function)

EPCF função poli-característica empírica (Empirical Poly-Characteristic Function)

EQM erro quadrático médio

GLS mínimos quadrados generalizados (Generalized Least Squares)

GMM método generalizado dos momentos (Generalized Method of Moments)

MDA mediana dos desvios absolutos

p. $\quad$ em probabilidade (in probability)

PCF função poli-característica (Poly-Characteristic Function)

QML quase máxima verossimilhança (Quasi-Maximum Likelihood)

SCD duração condicional estocástica (Stochastic Conditional Duration) 



\section{Capítulo 1}

\section{Introdução}

Em Probabilidade e Estatística é comum o uso de transformações para facilitar a solução de problemas considerados difíceis. Em particular, a transformada de Fourier da função densidade, a chamada função característica (CF), é uma das ferramentas mais utilizadas, pois permite a obtenção de resultados analíticos para diversos problemas, como a convolução de variáveis i.i.d., a obtenção de distribuições assintóticas de funções de variáveis aleatórias, etc. Por sua vez, a aplicação desta transformação à função de distribuição amostral resulta na chamada função característica empírica (ECF - Empirical Characteristic Function), que apresenta também várias vantagens, sumarizadas por Feuerverger e Mureika (1977):

"The range of problems to which the ecf seems applicable appears to be quite wide. This is because Fourier-Stieltjes transformation often results in easy translation of properties that are important in problems of inference. The characteristic function behaves simply under shifts, scale changes and summation of independent variables; it allows an easy characterization of independence and of symmetry. It is therefore not difficult to suggest ecf procedures for areas of inference such as testing for goodness of fit, testing for independence, parameter estimation, etc. Further, although it is true that there exist other functionals in one-to-one correspondence with the distributions, it may be argued that the characteristic function is a uniquely important concept for which a large body of theory is available. The ecf retains all information present in the sample and lends itself conveniently to computation. Finally, there are situations where a characterization of some property or of a class of distributions exists in terms of characteristic functions. One example is the problem of inference on the parameters of the stable laws. Here traditional methods have not led to a solution and an ecf approach seems likely to lead to useful procedures."

O uso da ECF em problemas de estimação parece ser bastante antigo: Montfort e Mooijaart (1989) citam um trabalho de Darmois $(1940)^{1}$, que teria usado

\footnotetext{
${ }^{1}$ Darmois, G., Les mathematiques de la psychologie, Memorial des sciences mathematiques, fasc. 48, Gautier-Villars, Paris, 1940.
} 
a ECF para estimação dos coeficientes em alguns modelos estatísticos simples, e citam outros autores que teriam usado a ECF para problemas com erros nas variáveis, como Neyman (1951) e Wolfowitz (1952) ${ }^{2}$. Na nossa pesquisa, entretanto, o documento mais antigo que obtivemos com citação da ECF foi o artigo de Rubin (1956), que aplica o resultado de um teorema de convergência de funções de variáveis aleatórias para ilustrar a convergência com probabilidade um da função característica empírica (amostral, na sua notação) para a função característica populacional no caso i.i.d. A citação seguinte que encontramos foi em Parzen (1962), em um problema de estimação não paramétrica da função densidade. Desde então a faixa de problemas de estimação para os quais a ECF tem sido utilizada é bastante ampla. Citamos por exemplo:

- Mistura de normais, como em Carrasco e Florens (2000a);

- Distribuições estáveis, como em Press (1972), Paulson et al. (1975), Leitch e Paulson (1975), Arad (1980), Koutrouvelis (1982), Feuerverger e McDunnough (1981a), Brockwell e Brown (1981), Paulson e Delehanty (1985), Brockwell e Liu (1992) e Kogon e Williams (1998);

- Processos ARMA estáveis, como em Knight e Yu (2002);

- Modelos de volatilidade estocástica, como em Knight et al. (2002) e Silva et al. (2004);

- Modelos afins de difusão, como em Singleton (2001), Jiang e Knight (2002), Chacko e Viceira (2003), Yu (2004) e Rockinger e Semenova (2005);

- Modelos com erros de medida, como em Montfort e Mooijaart (1989) e Hu e Ridder $(2006)$;

- Modelos lineares generalizados, como em Li e Hsiao (2004).

- Distribuições elípticas simétricas, como em Nakamura et al. (1996).

- Análise de Componentes Independentes (ICA - Independent Component Analysis), como em Chen e Bickel (2003).

A ECF também tem sido utilizada em problemas de teste de aderência de distribuições, como por exemplo:

- Forma de uma distribuição, como em Murota e Takeuchi (1981).

- Dados agrupados, como em Meintanis e Ushakov (2004);

- Mistura de normais, como em Klar e Meintanis (2005);

\footnotetext{
${ }^{2}$ Neyman, J., Existence of consistent estimates of the directional parameter in a linear structural relation between two variables, Annals of Mathematical Statistics, 22, 497-512, 1951.

Wolfowitz, J., Consistent estimators of the parameters of a linear structural relation, Skandinavisk Aktuarietidskrift, 35, 132-151, 1952.
} 
- Distribuições simétricas estáveis, como em Meintanis (2005);

- Distribuição Weibull e de valor extremo tipo I, como em Cabaña e Quiroz (2005).

Além desta modalidade, a ECF tem sido empregada em outras modalidades de testes:

- Simetria, como em Csörgo e Heathcote (1987);

- Normalidade, como em Epps e Pulley (1983) e Epps (1987);

- Independência e dependência serial, como em Hong (1999, 2000), Murata (2001), Bilodeau e de Micheaux (2005).

Feuerverger e Mureika (1977) examinam certas propriedades probabilísticas da ECF. Quase simultaneamente Heathcote (1977) e Thorton e Paulson (1977) obtêm a distribuição assintótica para o estimador que minimiza uma distância entre a ECF e a CF, a mesma distância utilizada por Press (1972) e Paulson et al. (1975) para estimação dos parâmetros de distribuições estáveis. O uso deste método neste caso justifica-se pela ausência de uma fórmula explícita para a função densidade de funções de distribuição estáveis (a não ser nos casos particulares $\alpha=1 / 2,1$ ou 2), tornando difícil o uso do método da máxima verossimilhança.

Uma revisão bastante completa do método da ECF para estimação encontra-se em Yu (2004), tanto para o caso i.i.d., quanto para o caso estacionário. O autor revê também diversas aplicações da ECF, variando de modelos de mistura até modelos de processos em tempo contínuo.

O objetivo deste trabalho é o de utilizar o método de estimação pela ECF, conforme proposto por Knight e Yu (2002), para estimar o modelo de duração condicional estocástica (SCD) proposto por Bauwens e Veredas (2004). A vantagem do uso deste método neste caso é permitir a eliminação das variáveis latentes de uma maneira natural ao calcularmos a função característica do processo (estacionário). Naturalmente as variáveis latentes também precisam ser estimadas, e para isso propusemos algumas alternativas.

O trabalho está estruturado em 9 capítulos, e diversos apêndices. No primeiro capítulo, revisamos a literatura sobre o uso da ECF em diversas áreas de aplicação. No segundo capítulo, apresentamos alguns resultados obtidos na literatura sobre a função característica (CF) e sua contraparte empírica (ECF). No terceiro capítulo, revemos os métodos propostos na literatura para uso da ECF em problemas de estimação. No quarto capítulo apresentamos o particular problema de duração estocástica condicional (SCD) que queremos estimar, e obtemos diversos resultados relativos ao processo. No quinto capítulo particularizamos os métodos de estimação da ECF para o nosso processo SCD. No sexto capítulo debruçamo-nos sobre o problema de estimação das variáveis latentes, propondo três alternativas: o filtro de Kalman, um filtro obtido por integração numérica (Bayesiano), e um filtro baseado na expansão de Gram-Charlier até quarta ordem. No sétimo capítulo aplicamos os resultados obtidos em amostras simuladas fixando-se os parâmetros. No oitavo capítulo aplicamos os 
resultados obtidos em uma amostra de durações de volume da GE (General Eletric) e Microsoft, proveniente de dados disponíveis publicamente. Concluimos o trabalho no capítulo 9, e acrescentamos nos apêndices diversos resultados e demonstrações. 


\section{Capítulo 2}

\section{A Função Característica Empírica}

Neste capítulo vamos inicialmente rever a definição e algumas propriedades da função característica, que poderão vir a ser úteis no desenvolvimento do texto. Em seguida, vamos definir a função característica empírica e apresentar algumas de suas propriedades, separadas em duas categorias: processos independentemente igualmente distribuídos (i.i.d.) e processos estacionários.

\subsection{A Função Característica (CF)}

A Função Característica (CF - Characteristic Function) é definida como a transformada de Fourier-Stieltjes da função distribuição de probabilidade. Como uma transformação da distribuição de probabilidade sua maior vantagem reside no fato de sempre existir, ao contrário, por exemplo, da Função Geratriz de Momentos.

A seguir vamos rever sua definição e algumas de suas propriedades, seguindo para isso o texto de Shirvaev (1991). Ao longo do texto vamos denotar o número imaginário por $i=\sqrt{-1}$.

Definição 2.1 (Shiryaev (1991), pg. 274) Seja $F=F\left(x_{1}, \cdots, x_{k}\right)$ uma função distribuição k-dimensional em $\left(\mathbb{R}^{k}, \mathcal{B}\left(\mathbb{R}^{k}\right)\right)$. Sua função característica é dada por

$$
\varphi(r)=\int_{\mathbb{R}^{k}} e^{i r^{\prime} x} d F(x), \quad r \in \mathbb{R}^{k}
$$

Alternativamente, se $\xi=\left(\xi_{1}, \cdots, \xi_{k}\right)^{\prime}$ for um vetor aleatório definido no espaço de probabilidades $(\Omega, \mathcal{F}, P)$ com valores em $\mathbb{R}^{k}$, sua função característica é

$$
\varphi_{\xi}(r)=\int_{\mathbb{R}^{k}} e^{i r^{\prime} x} d F_{\xi}(x), \quad r \in \mathbb{R}^{k}
$$

onde $F_{\xi}=F_{\xi}\left(x_{1}, \cdots, x_{k}\right)$ é a função distribuição do vetor $\xi=\left(\xi_{1}, \cdots, \xi_{k}\right)^{\prime}$, i.e., $F_{\xi}\left(x_{1}, \cdots, x_{k}\right)=P\left[X_{1} \leq x_{1}, \cdots, X_{k} \leq x_{k}\right]$. 
Se $F(x)$ possuir uma densidade $f=f(x)$ então

$$
\varphi(r)=\int_{\mathbb{R}^{k}} e^{i r^{\prime} x} f(x) d x, \quad r \in \mathbb{R}^{k}
$$

Neste caso, a função característica é simplesmente a transformada de Fourier de $f(x)$. Note também que a função característica pode ser definida como o seguinte valor esperado

$$
\varphi_{\xi}(r)=E\left[e^{i r^{\prime} \xi}\right], \quad r \in \mathbb{R}^{k}
$$

\subsubsection{Algumas propriedades da CF}

Vamos rever agora algumas propriedades básicas das funções características. Seja $\xi=\xi(\omega)$ uma variável aleatória com função distribuição $F=F_{\xi}(x)$ e com função característica $\varphi_{\xi}(r)=E\left[e^{i r^{\prime} \xi}\right]$. Seja $A$ uma matriz $m \times k$ e $b$ um vetor $m \times 1$. Para uma variável aleatória dada por $\eta=A \xi+b$ então

$$
\varphi_{\eta}(r)=E\left[e^{i r^{\prime} \eta}\right]=E\left[e^{i r^{\prime}(A \xi+b)}\right]=e^{i r^{\prime} b} E\left[e^{i r^{\prime} A \xi}\right]=e^{i r^{\prime} b} E\left[e^{i\left(A^{\prime} r\right)^{\prime} \xi}\right] .
$$

Portanto,

$$
\varphi_{\eta}(r)=e^{i r^{\prime} b} \varphi_{\xi}\left(A^{\prime} r\right) .
$$

Se $\xi_{1}, \cdots, \xi_{n}$ forem variáveis aleatórias independentes e $S_{n}=\xi_{1}+\cdots+\xi_{n}$, então

$$
\begin{aligned}
\varphi_{S_{n}}(r) & =E\left[e^{i r^{\prime} S_{n}}\right]=E\left[e^{i r^{\prime}\left(\xi_{1}+\cdots+\xi_{n}\right)}\right]=E\left[e^{i r^{\prime} \xi_{1}+\cdots+i r^{\prime} \xi_{n}}\right]= \\
& =E\left[e^{i r^{\prime} \xi_{1}}\right] \ldots E\left[e^{i r^{\prime} \xi_{n}}\right]=\varphi_{\xi_{1}}(r) \ldots \varphi_{\xi n}(r) .
\end{aligned}
$$

Logo,

$$
\varphi_{S_{n}}(r)=\prod_{j=1}^{n} \varphi_{\xi_{j}}(r)
$$

Ou seja, a função característica de uma soma de variáveis aleatórias independentes é dada pelo produto da funções características de cada uma delas.

Outras propriedades da função característica são apresentadas no seguinte teorema de Shirvaev (1991), que apresentaremos sem a demonstração.

Teorema 2.1 (Shiryaev (1991), pg. 278) Seja $\xi$ uma variável aleatória com função distribuição $F=F(x)$ e função característica $\varphi(r)=E\left[e^{i r \xi}\right]$. Então $\varphi$ tem as seguintes propriedades:

(a) $|\varphi(r)| \leq|\varphi(0)|=1$;

(b) $\varphi(r)$ é uniformemente contínua para $r \in \mathbb{R}$; 
(c) $\varphi(r)=\overline{\varphi(-r)}$;

(d) $\varphi(r)$ é real se e somente $F$ for simétrica $\left(\int_{A} d F(x)=\int_{-A} d F(x), A \in \mathcal{B}(\mathbb{R}),-A=\right.$ $\{-x: x \in A\})$;

(e) Se $E\left[|\xi|^{n}\right]<\infty$ para algum $n \geq 1$, então $\varphi^{(k)}(r)$ existe para todo $k \leq n$, e

$$
\begin{gathered}
\varphi^{(k)}(r)=\int_{\mathbb{R}}(i x)^{k} e^{i t x} d F(x), \\
E\left[\xi^{k}\right]=\frac{\varphi^{(k)}(0)}{i^{k}}, \\
\varphi(r)=\sum_{k=0}^{n} \frac{(i r)^{k}}{k !} E\left[\xi^{k}\right]+\frac{(i r)^{n}}{n !} \varepsilon_{n}(r),
\end{gathered}
$$

onde $\left|\varepsilon_{n}(r)\right| \leq 3 E\left[\xi^{n}\right]$ e $\varepsilon_{n}(r) \rightarrow 0$ quando $r \rightarrow 0 ;$

(f) $\operatorname{Se} \varphi^{(2 n)}(0)$ existir e for finito então $E\left[\xi^{2 n}\right]<\infty$.

Prova. Ver Shiryaed (1991), pg. 278.

Para uma revisão de outras propriedades da função característica e de suas diversas aplicações sugerimos Lukacs (1970) e, também, Lukacs (1983).

\subsection{A Função Característica Empírica (ECF)}

A Função Caraterística Empírica (ECF - Empirical Characteristic Function), também chamada de função característica amostral, é simplesmente a transformada de Fourier-Stieltjes da Função Distribuição Empírica (ou amostral). Assim, vamos inicialmente definir a função distribuição empírica:

Definição 2.2 (Billingsley (1995), pg. 268 ) Sejam $X_{1}, \cdots, X_{n}$ variáveis aleatórias com função distribuição $F(x)$. A função distribuição empírica (ou amostral) é dada por

$$
F_{n}(x, \omega)=\frac{1}{n} \sum_{j=1}^{n} \mathbb{I}_{(-\infty, x]}\left(X_{j}(\omega)\right) .
$$

Na definição acima usamos a função indicadora definida por:

$$
\mathbb{I}_{A}(x)=\left\{\begin{array}{l}
1, \text { se } x \in A \\
0, \text { se } x \notin A .
\end{array} .\right.
$$

Dada uma amostra de variáveis aleatórias com mesma distribuição $F$, podemos estimar a sua função característica em (2.1) se utilizarmos a função distribuição empírica $F_{n}$ em (2.7) como estimador de $F$. 
Definição 2.3 (Função Característica Empírica - ECF) Sejam $X_{1}, \cdots, X_{n}$ variáveis aleatórias $k$-dimensionais com distribuição $F(x)$. A função característica empírica (ou amostral) é uma variável aleatória complexa, função de $r \in \mathbb{R}^{k}$, dada por

$$
\varphi_{n}(r)=\frac{1}{n} \sum_{j=1}^{n} e^{i r^{\prime} X_{j}}
$$

É interessante notar que (2.8) pode ser reescrita da seguinte maneira:

$$
\begin{aligned}
\varphi_{n}(r) & =\frac{1}{n} \sum_{j=1}^{n} \cos \left(r^{\prime} X_{j}\right)+i \frac{1}{n} \sum_{j=1}^{n} \sin \left(r^{\prime} X_{j}\right) \\
& =\operatorname{Re} \varphi_{n}(r)+i \operatorname{Im} \varphi_{n}(r) .
\end{aligned}
$$

Podemos notar pela relação (2.8) que existe uma correspondência biunívoca entre a função distribuição empírica e a função característica empírica. Como a ECF contém toda a informação da amostra, é natural utilizá-la para estimação de parâmetros. Para isso, vamos examinar a seguir algumas das propriedades da ECF como obtidas por Feuerverger e Mureika (1977) e Feuerverger (1990). Outros resultados de convergência para a ECF podem ser encontrados em Kellermeier (1980), Csörgo (1981), Marcus (1981), Stute (1983), Yukich (1985), Lacey (1989), Hesse (1990) e Wells (1992).

\subsection{Propriedades da ECF}

\subsubsection{Séries de v.a. independentes e identicamente distribuidas (i.i.d.)}

Para variáveis aleatórias $X_{1}, \cdots, X_{n}$ identicamente distribuídas (não necessariamente independentes) o valor esperado da função característica empírica coincide com a função característica de $X_{j}$, pois

$$
E\left[\varphi_{n}(r)\right]=\frac{1}{n} \sum_{j=1}^{n} E\left[e^{i r^{\prime} X_{j}}\right]=\frac{1}{n} \sum_{j=1}^{n} \varphi(r)=\varphi(r) .
$$

Seja agora $X_{1}, \cdots, X_{n}$ uma amostra aleatória de variáveis i.i.d. Para $r$ fixo, $\varphi_{n}(r)$ é uma média de variáveis aleatórias limitadas i.i.d., com média $\varphi(r)$. Logo, segue da Lei Forte dos Grandes Números que $\varphi_{n}(r)$ converge quase certamente para $\varphi(r)$ :

$$
\varphi_{n}(r) \stackrel{a . s .}{\longrightarrow} \varphi(r),
$$

isto é,

$$
\operatorname{Pr}\left[\lim _{n \rightarrow \infty} \varphi_{n}(r)=\varphi(r)\right]=1 .
$$

Além disso, Feuerverger e Mureika (1977) obtiveram os seguintes resultados: 
Teorema 2.2 (Feuerverger e Mureika (1977), Teor. 2.1) Para fixo $R<\infty$,

$$
\sup _{|r| \leq R}\left|\varphi_{n}(r)-\varphi(r)\right| \stackrel{a . s .}{\longrightarrow} 0
$$

isto é,

Prova. Ver Feuerverger e Mureika (1977), teorema 2.1, pg.89.

$$
\operatorname{Pr}\left[\lim _{n \rightarrow \infty} \sup _{|r| \leq R}\left|\varphi_{n}(r)-\varphi(r)\right|=0\right]=1 .
$$

Teorema 2.3 (Feuerverger e Mureika (1977), Teor. 3.1) Seja $Y_{n}(r)$ o processo aleatório complexo dado por:

$$
Y_{n}(r)=\sqrt{n}\left(\varphi_{n}(r)-\varphi(r)\right) .
$$

Note que $E\left[Y_{n}(r)\right]=0, \operatorname{Cov}\left[Y_{n}(r), Y_{n}(s)\right]=E\left[Y_{n}(r) \cdot \overline{Y_{n}(s)}\right]=\varphi(r-s)-$ $\varphi(r) . \varphi(-s)$, ou analogamente, $E\left[Y_{n}(r) . Y_{n}(s)\right]=\varphi(r+s)-\varphi(r) \cdot \varphi(s)$. Também:

$$
\begin{aligned}
& \operatorname{Cov}[\operatorname{Re} Y(r), \operatorname{Re} Y(s)]=\frac{1}{2}[\operatorname{Re} \varphi(r+s)+\operatorname{Re} \varphi(r-s)]-\operatorname{Re} \varphi(r) \operatorname{Re} \varphi(r), \\
& \operatorname{Cov}[\operatorname{Re} Y(r), \operatorname{Im} Y(s)]=\frac{1}{2}[\operatorname{Re} \varphi(r+s)-\operatorname{Im} \varphi(r-s)]-\operatorname{Re} \varphi(r) \operatorname{Im} \varphi(r), \\
& \operatorname{Cov}[\operatorname{Im} Y(r), \operatorname{Im} Y(s)]=\frac{1}{2}[-\operatorname{Re} \varphi(r+s)+\operatorname{Re} \varphi(r-s)]-\operatorname{Im} \varphi(r) \operatorname{Im} \varphi(r) .
\end{aligned}
$$

Defina $Y(r)$ como o processo estocástico Gaussiano complexo de média zero, com mesma estrutura de covariância de $Y_{n}(r)$ e satisfazendo $Y(r)=\overline{Y(-r)}$. Então, o processo $Y_{n}(r)$ converge fracamente para $Y(r)$ em cada intervalo finito.

Prova. A demonstração deste teorema encontra-se em Feuerverger e Mureika (1977), teorema 3.1, pg.92. No apêndice B obtemos a estrutura de covariância para processos i.i.d. apresentada no teorema.

\subsubsection{Séries estacionárias}

Os resultados da seção anterior foram obtidos para o caso de variáveis aleatórias identicamente e independentemente distribuídas, o que limita o seu emprego para análise de séries temporais, onde comumente observamos dependência serial entre as observações. Para lidar com o caso de séries univariadas ergódicas estritamente estacionárias Feuerverger e McDunnough (1981a) propuseram o uso de uma "função poli-característica" e correspondente "função policaracterística empírica", obtida da seguinte forma: seja $\left\{X_{j}\right\}_{j=-\infty}^{\infty}$ uma série temporal univariada estritamente estacionária cuja distribuição depende de um vetor de parâmetros $\theta$. Defina o seguinte vetor $(p+1) \times 1$, construído como um bloco sequencial de tamanho $p$ das variáveis $X_{j}$ :

$$
Z_{j}=\left(X_{j}, X_{j+1}, \cdots, X_{j+p}\right)^{\prime} .
$$


Sua "função poli-característica" (PCF - Poly-Characteristic Function) é definida em função de $r=\left(r_{0}, \cdots, r_{p}\right)^{\prime} \in \mathbb{R}^{p+1}$ por:

$$
\varphi(r ; \theta)=E\left[e^{i r^{\prime} Z_{j}}\right]
$$

A correspondente "função poli-característica empírica" (EPCF - Empirical PolyCharacteristic Function) é definida por:

$$
\varphi_{n}(r)=\frac{1}{n} \sum_{j=1}^{n} e^{i r^{\prime} Z_{j}}
$$

Como a seqüência $\left\{Z_{j}\right\}_{j=\infty}^{\infty}$ possui dependência serial por construção, não é possível aplicar os resultados de Feuerverger e Mureika (1977). Para obter resultados de convergência para a EPCF Feuerverger (1990) exige algumas condições mixing, que descrevemos a seguir. Defina a correlação máxima $\rho(q)$ como o supremo da correlação entre variáveis aleatórias de quadrado integrável mensuráveis com relação às sigma-álgebras $\sigma\left\{\cdots, X_{-1}, X_{0}\right\} \mathrm{e}$ $\sigma\left\{X_{q}, X_{q+1}, \cdots\right\}$ respectivamente, i.e., $\rho(q)$ é a correlação máxima possível entre funções de $X_{j}$ 's separadas no tempo por uma distância $q$. Feuerverger (1990) requer a condição abaixo nos teoremas que apresentaremos a seguir, mas enfatiza que esta condição poderia ser trocada pela condição mais fraca de ergodicidade e mixing "forte" :

$$
\sum_{q=1}^{\infty} \rho(q)<\infty
$$

Teorema 2.4 (Feuerverger (1990), Teor.2.1) Se $\left\{X_{j}\right\}_{j=-\infty}^{\infty}$ for uma série temporal estritamente estacionária satisfazendo (2.14), então

(a) $\varphi_{n}(r) \rightarrow \varphi(r ; \theta)$ quase certamente, para todo $r \in \mathbb{R}^{p+1}$.

(b) $\sup _{-T<r^{0}, \ldots, r^{p}<T}\left|\varphi_{n}(r)-\varphi(r ; \theta)\right| \rightarrow 0$ quase certamente, para todo $T$ fixo, $0<T<\infty$

Prova. Ver Feuerverger (1990), Teor.2.1.

Feuerverger (1990) também obtém o seguinte resultado de convergência em distribuição para a EPCF:

Teorema 2.5 (Feuerverger (1990), Teor.2.2) Assuma que $\left\{X_{j}\right\}_{j=-\infty}^{\infty}$ satisfaça (2.14), e seja $Y(r), r \in \mathbb{R}^{p+1}$, um processo Gaussiano complexo de média zero e com estrutura de covariância dada por

$$
\lim _{n \rightarrow \infty} \operatorname{cov}\left[Y_{n}(r), Y_{n}(s)\right]=\sum_{k=-\infty}^{\infty} \operatorname{cov}\left[e^{i r^{\prime} Z_{0}}, e^{i s^{\prime} Z_{k}}\right],
$$

\footnotetext{
${ }^{1}$ No apêndice A revisamos alguns conceitos de mixing.
} 
onde $Y_{n}(r)=\sqrt{n}\left(\varphi_{n}(r)-\varphi(r)\right)$. Então $\left(Y_{n}\left(r_{1}\right), \cdots, Y_{n}\left(r_{m}\right)\right)$ converge em distribuição para $\left(Y\left(r_{1}\right), \cdots, Y\left(r_{m}\right)\right)$, para todo $m$ e todos $r_{1}, r_{2}, \cdots, r_{m} \in \mathbb{R}^{p+1}$.

Prova. Ver Feuerverger (1990), Teor.2.2.

Podemos obter também a matriz de covariância do processo $Y_{n}(r)=\sqrt{n}\left(\varphi_{n}(r)-\varphi(r)\right)$, através da seguinte proposição, análoga a apresentada por Knight e Satchell (1997):

Proposição 2.1 (Covariância de $Y_{n}$ ) Seja $\left\{Z_{j}\right\}_{j=-\infty}^{\infty}$ um processo estocástico estritamente estacionário $p+1$ variado, com função característica $\varphi(r)$ e respectiva função característica empírica $\varphi_{n}(r)$, e seja $Y_{n}(r)$ o processo aleatório complexo dado por

$$
Y_{n}(r)=\sqrt{n}\left(\varphi_{n}(r)-\varphi(r)\right)
$$

Note que $E\left[Y_{n}(r)\right]=0$. Seja $\Lambda_{k}: \mathbb{R}^{p+1} \times \mathbb{R}^{p+1} \longmapsto \mathbb{C}$ a função definida por

$$
\Lambda_{k}(r, s)=E\left[e^{i r^{\prime} Z_{j}+i s^{\prime} Z_{j+k}}\right] .
$$

A covariância entre as partes real e imaginária de $Y_{n}$ é dada por

$$
\Omega_{n}(r, s)=\left(\begin{array}{cc}
\Omega_{R R, n}(r, s) & \Omega_{R I, n}(r, s) \\
\Omega_{I R, n}(r, s) & \Omega_{I I, n}(r, s)
\end{array}\right),
$$

onde

$$
\begin{aligned}
\Omega_{R R, n}(r, s) & =\operatorname{Cov}[\operatorname{Re} Y(r), \operatorname{Re} Y(s)] \\
\Omega_{R I, n}(r, s) & =\operatorname{Cov}[\operatorname{Re} Y(r), \operatorname{Im} Y(s)] \\
\Omega_{I R, n}(r, s) & =\operatorname{Cov}[\operatorname{Im} Y(r), \operatorname{Re} Y(s)] \\
\Omega_{I I, n}(r, s) & =\operatorname{Cov}[\operatorname{Im} Y(r), \operatorname{Im} Y(s)]
\end{aligned}
$$


$e$

$$
\begin{aligned}
\Omega_{R R, n}(r, s)= & \frac{1}{2}\{\operatorname{Re} \varphi(r-s)+\operatorname{Re} \varphi(r+s)\}-n \operatorname{Re} \varphi(r) \operatorname{Re} \varphi(s)+ \\
& +\frac{1}{2 n} \sum_{k=1}^{n-1}(n-k)\left\{\operatorname{Re} \Lambda_{k}(r,-s)+\operatorname{Re} \Lambda_{k}(r, s)+\operatorname{Re} \Lambda_{k}(-s, r)+\operatorname{Re} \Lambda_{k}(s, r)\right\}
\end{aligned}
$$$$
\Omega_{R I, n}(r, s)=\frac{1}{2}\{-\operatorname{Im} \varphi(r-s)+\operatorname{Im} \varphi(r+s)\}-n \operatorname{Re} \varphi(r) \operatorname{Im} \varphi(s)+
$$$$
+\frac{1}{2 n} \sum_{k=1}^{n-1}(n-k)\left\{-\operatorname{Im} \Lambda_{k}(r,-s)+\operatorname{Im} \Lambda_{k}(r, s)-\operatorname{Im} \Lambda_{k}(-s, r)+\operatorname{Im} \Lambda_{k}(s, r)\right\}
$$

$$
\begin{aligned}
\Omega_{I R, n}(r, s)= & \frac{1}{2}\{\operatorname{Im} \varphi(r-s)+\operatorname{Im} \varphi(r+s)\}-n \operatorname{Im} \varphi(r) \operatorname{Re} \varphi(s)+ \\
& +\frac{1}{2 n} \sum_{k=1}^{n-1}(n-k)\left\{\operatorname{Im} \Lambda_{k}(r,-s)+\operatorname{Im} \Lambda_{k}(r, s)+\operatorname{Im} \Lambda_{k}(-s, r)+\operatorname{Im} \Lambda_{k}(s, r)\right\}
\end{aligned}
$$

$$
\begin{aligned}
\Omega_{I I, n}(r, s)= & \frac{1}{2}\{\operatorname{Re} \varphi(r-s)-\operatorname{Re} \varphi(r+s)\}-n \operatorname{Im} \varphi(r) \operatorname{Im} \varphi(s)+ \\
& +\frac{1}{2 n} \sum_{k=1}^{n-1}(n-k)\left\{\operatorname{Re} \Lambda_{k}(r,-s)-\operatorname{Re} \Lambda_{k}(r, s)+\operatorname{Re} \Lambda_{k}(-s, r)-\operatorname{Re} \Lambda_{k}(s, r)\right\}
\end{aligned}
$$

Prova. A demonstração desta proposição encontra-se no apêndice B.

\subsection{Sumário}

Neste capítulo relembramos a definição da função característica $\varphi(r)$ e algumas de suas propriedades. Vimos também a definição da função característica empírica (ECF), $\varphi_{n}(r)$, e apresentamos alguns resultados obtidos por Feuerverger e Mureika (1977), Feuerverger (1990) e Knight e Satchell (1997) para a convergência do processo $\sqrt{n}\left(\varphi_{n}(r)-\varphi(r)\right)$. No capítulo seguinte vamos revisar alguns métodos de estimação de parâmetros que empregam a ECF. 


\section{Capítulo 3}

\section{Métodos de Estimação utilizando a ECF}

Os métodos de estimação que utilizam a ECF citados na literatura adotam basicamente duas abordagens: maximização de função verossimilhança ou minimização de alguma distância entre a ECF e a CF.

Entre as abordagens que adotam maximização de função verossimilhança encontramos o método " $k-L$ " de Feuerverger e McDunnough (1981b) e o método "ML-CCF" de Singleton (2001). O método " $k-L$ " de Feuerverger e McDunnough (1981b) procura maximizar a verossimilhança obtida a partir da distribuição assintótica de $Y_{n}(r)$ que, como vimos no capítulo anterior, tem distribuição assintótica conhecida. Já o método "ML-CCF" de Singleton (2001, 2006) procura maximizar a função de verossimilhança obtida a partir da inversão de Fourier da função característica condicional ( $\mathrm{CCF})$.

No entanto a maior parte dos autores revisados procura minimizar alguma distância entre a ECF e CF, utilizando uma abordagem de estimadores extremos (Amemiva (1985)), quando o parâmetro $r$ é contínuo (e.g., Press (1972), Paulson et al. (1975), Heathcote (1977), Knight e Yu (2002), Knight et al. (2002), Yu (2004)), ou uma abordagem de método dos momentos, quando o parâmetro $r$ é discreto (e.g., Feuerverger e McDunnough (1981b), Singleton (2001), Jiang e Knight (2001), Chacko e Viceira (2003)), e até usando ambas abordagens como por exemplo, Jiang e Knight (2002). Knight e Yu (2002) denominam o caso de $r$ contínuo de método da ECF contínua (CECF-Continuous ECF) e o caso de $r$ discreto de método da ECF discreta (DECF - Discrete ECF).

Mais recentemente, entretanto, Carrasco e Florens (2000b), estendem o método generalizado dos momentos (GMM) de Hansen (1982) para um "contínuo" de momentos, e empregam este estimador em Carrasco e Florens (2002) para obter estimadores "eficientes" utilizando a ECF.

No apêndice A revisamos alguns resultados para estimadores extremos seguindo para isso Amemiya (1985). Como o método da ECF discreta é um caso particular do método dos momentos, revisamos também no mesmo apêndice o método generalizado dos momentos 
(GMM) de Hansen (1982).

No seção a seguir vamos revisar os resultados obtidos por Knight e Yu (2002) para o caso de séries estacionárias (não necessariamente i.i.d.) ${ }^{1}$. Na outra seção vamos abordar o problema segundo o método dos momentos, e a seguir vamos também rever os resultados obtidos por Carrasco e Florens (2002).

\subsection{O método da ECF contínua (CECF)}

Press (1972) e Paulson et al. (1975) aparentemente foram os primeiros autores a propor um estimador que minimizasse uma distância entre a função característica empírica e a função característica, de maneira a eliminar o parâmetro $r$ :

$$
Q_{n}(\theta)=\int\left|\varphi_{n}(r)-\varphi(r ; \theta)\right|^{\nu} d G(r) .
$$

Quando $\nu=2$ (erro quadrático médio integrado) Heathcote (1977) obtém resultados de convergência para o caso de processos identicamente e independentemente distribuídos, enquanto Knight e Yu (2002) obtêm resultados para o caso de processos estacionários.

Knight e Yu (2002) propõem o estimador da ECF contínua (CECF - Continuous ECF) que minimiza a integral

$$
Q_{n}(\theta)=\int\left|\varphi_{n}(r)-\varphi(r ; \theta)\right|^{2} d G(r)
$$

$\mathrm{ou}$

$$
Q_{n}(\theta)=\int\left|\varphi_{n}(r)-\varphi(r ; \theta)\right|^{2} g(r) d r
$$

ou que resolve a seguinte equação de estimação

$$
\int w_{\theta}(r)\left(\varphi_{n}(r)-\varphi(r ; \theta)\right) d r=0
$$

em que $G(r), g(r)$ e $w_{\theta}(r)$ são funções de peso apropriadas. Sob condições de regularidade apropriadas os três métodos são equivalentes. Feuerverger (1990) mostra que é possível escolher uma função de peso $w_{\theta}^{*}(r)$ de maneira que o estimador em (3.4) alcance eficiência arbitrariamente alta, desde que $p$ seja suficientemente grande:

$$
w_{\theta}^{*}(r)=\left(\frac{1}{2 \pi}\right)^{p+1} \int \cdots \int e^{-i r^{\prime} X_{j}} \frac{\partial \ln f\left(y_{j+p} \mid y_{j}, \cdots, y_{j+p-1}\right)}{\partial \theta} d y_{1} \cdots d y_{j+p}
$$

Note que o peso ótimo $w_{\theta}^{*}(r)$ depende da função densidade de probabilidade condicional $f\left(y_{j+p} \mid y_{j}, \cdots, y_{j+p-1}\right)$, que pode não ser conhecida ou ser de difícil obtenção.

Entretanto a escolha de $G(r), g(r)$ e $w_{\theta}(r)$ é feita na maioria das vezes de maneira a facilitar o procedimento de integração numérica. Em particular, se escolhermos $g(r)=$

\footnotetext{
${ }^{1} \mathrm{O}$ caso i.i.d. é tratado por Heathcote (1977) e Thorton e Paulson (1977).
} 
$\exp \left(-r^{\prime} r\right)$ podemos empregar o método da quadratura de Gauss-Hermite (veja, por exemplo, Press et al. (1997)).

Vamos apresentar agora o resultado obtido por Knight e Yu (2002) para séries estacionárias (não necessariamente i.i.d.). Para isso, seja $\left\{X_{j}\right\}_{j=-\infty}^{\infty}$ uma série temporal univariada estritamente estacionária cuja distribuição depende de um vetor de parâmetros $\theta$ e seja $Y_{j}$ o vetor de tamanho $p+1$, formado por blocos de $X_{j}$, definido em (2.11) como:

$$
Y_{j}=\left(X_{j}, X_{j+1}, \cdots, X_{j+p}\right)^{\prime} .
$$

Fixado um $p$, Knight e Yu (2002) definem as seguintes condições de regularidade:

(a) $\theta \in \Theta$, onde o espaço paramétrico $\Theta \subset \mathbb{R}^{k}$ é um conjunto compacto com $\theta_{0} \in$ interior $(\Theta)$.

(b) Com probabilidade um, $Q_{n}(\theta)$ é diferenciável continuamente duas vezes sob o sinal de integração com respeito a $\theta$ em $\Theta$.

(c) A seqüência $\left\{Y_{n}\right\}$ é estritamente estacionária e ergódica.

(d) Seja

$$
Q_{0}(\theta)=\int\left|\varphi\left(r ; \theta_{0}\right)-\varphi(r ; \theta)\right|^{2} d G(r)
$$

e $Q_{0}(\theta)=0$ somente se $\theta=\theta_{0}$.

(e) $K(y ; \theta)$ é uma função mensurável e limitada de $y$ para todo $\theta$, onde

$$
\begin{aligned}
K(y ; \theta)= & \int\left\{\left(\cos \left(r^{\prime} y\right)-\operatorname{Re} \varphi(r ; \theta)\right) \frac{\partial \operatorname{Re} \varphi(r ; \theta)}{\partial \theta}+\right. \\
& \left.+\left(\sin \left(r^{\prime} y\right)-\operatorname{Im} \varphi(r ; \theta)\right) \frac{\partial \operatorname{Im} \varphi(r ; \theta)}{\partial \theta}\right\} d G(r) .
\end{aligned}
$$

(f) $B\left(\theta_{0}\right)$ é não singular, onde

$$
B\left(\theta_{0}\right)=\int \frac{\partial \varphi\left(r ; \theta_{0}\right)}{\partial \theta} \frac{\partial \varphi\left(r ; \theta_{0}\right)}{\partial \theta^{\prime}} d G(r)
$$

e $\partial^{2} \varphi\left(r ; \theta_{0}\right) / \partial \theta \partial \theta^{\prime}$ é limitada uniformemente por uma função $G$-integrável em $\Theta$.

(g) Seja $\mathcal{F}_{j}$ uma $\sigma$-álgebra tal que $\left\{K_{j}, \mathcal{F}_{j}\right\}$ seja uma seqüência estocástica adaptada, onde $K_{j}=K\left(Y_{j} ; \theta\right)$. Podemos pensar em $\mathcal{F}_{j}$ como a $\sigma$-álgebra gerada pela história completa corrente e passada de $K_{j}$. Seja $\nu_{j}=E\left[K_{0} \mid K_{-j}, K_{-j-1}, \ldots\right]-E\left[K_{0} \mid K_{-j-1}, K_{-j-2}, \ldots\right]$ para $j \geq 0$. Suponha que $E\left[K_{0} \mid \mathcal{F}_{-m}\right]$ convirja em média quadrática para 0 quando $m \rightarrow \infty$ e $\sum_{j=0}^{\infty} E\left[\nu_{j}^{\prime} \nu_{j}\right]^{1 / 2}<\infty$.

(h) $G(r)$ é uma função não decrescente com variação total limitada igual a 1. 
As condições de regularidade acima são justificadas por Knight e Yu (2002) da seguinte maneira: (a) garante que o espaço paramétrico seja compacto, enquanto (b) garante a continuidade de $Q_{n}(\theta)$. As hipóteses (c) e de (e) a (g) fornecem condições suficientes para uma lei dos grandes números e um teorema central do limite. De acordo com (h), $G(r)$ pode ser uma função distribuição; entretanto, uma função de peso constante não pode ser usada (por exemplo, $G(r)=a r$, para uma constante fixa $a$ ). A hipótese (g) é análoga à hipótese (3.5) em Hansen (1982) e vale sob condições mixing adequadas . A hipótese (d) é uma condição de identificação; sem ela, o estimador poderia ser inconsistente. Por exemplo para um processo $\operatorname{MA}(10), X_{t}=\varepsilon_{t}-\phi \varepsilon_{t-10}$, os blocos móveis de tamanho $p<10$ não contém informação sobre $\phi$, e assim o método leva a estimativas inconsistentes. Este caso é eliminado pela hipótese (d), mas a identificação somente é possível com uma escolha apropriada de $p$, que no caso do exemplo é $p \geq 10$. Sob estas condições os autores obtém o seguinte resultado de convergência assintótica para seu estimador:

Teorema 3.1 (Knight e Yu (2002), Teor. 2.1) Seja $\widehat{\theta}_{n}=\arg \min _{\theta \in \Theta} Q_{n}(\theta)$. Suponha que sejam válidas as hipóteses (a) a (d) e (h); então $\widehat{\theta}_{n} \stackrel{a . s .}{\rightarrow} \theta_{0}$.

Se além disso as hipóteses (e) a (g) forem válidas, então

$$
\sqrt{n}\left(\widehat{\theta}_{n}-\theta_{0}\right) \stackrel{d}{\longrightarrow} N\left(0, B\left(\theta_{0}\right)^{-1} A\left(\theta_{0}\right) B\left(\theta_{0}\right)^{-1}\right),
$$

em que

$$
A\left(\theta_{0}\right)=\operatorname{var}\left(K\left(X_{1} ; \theta_{0}\right)\right)+2 \sum_{j=2}^{\infty} \operatorname{cov}\left(K\left(X_{1} ; \theta_{0}\right), K\left(X_{j} ; \theta_{0}\right)\right) .
$$

Prova. Ver Knight e Yu (2002), Teor. 2.1, pg. 714. No apêndice D utilizamos os resultados de estimadores extremos para verificar a convergência do estimador CECF.

Para calcular uma estimativa para a matriz de covariância do estimador CECF é necessário obter estimativas para $A\left(\theta_{0}\right)$ e $B\left(\theta_{0}\right)$. Knight e Yu (2002) sugerem que a matriz $B\left(\theta_{0}\right)$ possa ser estimada consistentemente por $B\left(\widehat{\theta}_{n}\right)$, enquanto que um estimador consistente de $A\left(\theta_{0}\right)$ possa ser obtido com estimadores robustos a heteroscedasticidade e autocorrelação como em Andrews (1991) e Newey e West (1994). Não está claro no texto, mas possivelmente os autores estão sugerindo o uso de $K\left(y_{j} ; \widehat{\theta}_{n}\right)$ para estimação de $A\left(\theta_{0}\right)$. No apêndice D sugerimos algumas alternativas para estimação da matriz $A\left(\theta_{0}\right)$.

\subsection{O método da ECF discreta (DECF)}

O método da ECF discreta (DECF) procura minimizar uma distância entre a ECF e a $\mathrm{CF}$, fixada uma malha de $q$ pontos $r_{1}, \cdots, r_{q}$, constituindo-se em uma aplicação do método generalizado dos momentos (GMM). Para empregar este método vamos denotar:

$$
h\left(r, X_{j} ; \theta\right)=e^{i r^{\prime} X_{j}}-\varphi(r ; \theta) .
$$


Note que $E\left[e^{i r^{\prime} X_{j}}-\varphi(r ; \theta)\right]=0, \forall r \in \mathbb{R}^{p}$. Vamos definir agora o seguinte vetor $2 q \times 1$ :

$$
f\left(X_{j} ; \theta\right)=\left(\begin{array}{c}
\operatorname{Re} h\left(r_{1}, X_{j} ; \theta\right) \\
\vdots \\
\operatorname{Re} h\left(r_{q}, X_{j} ; \theta\right) \\
\operatorname{Im} h\left(r_{1}, X_{j} ; \theta\right) \\
\vdots \\
\operatorname{Im} h\left(r_{q}, X_{j} ; \theta\right)
\end{array}\right)_{2 q \times 1}
$$

Para obter o estimador GMM vamos definir os seguintes vetores $2 q \times 1$ :

$$
\begin{gathered}
V_{n}=\left(\begin{array}{c}
\operatorname{Re} \varphi_{n}\left(r_{1} ; \theta\right) \\
\vdots \\
\operatorname{Re} \varphi_{n}\left(r_{q} ; \theta\right) \\
\operatorname{Im} \varphi_{n}\left(r_{1} ; \theta\right) \\
\vdots \\
\operatorname{Im} \varphi_{n}\left(r_{q} ; \theta\right)
\end{array}\right)_{2 q \times 1} \\
V_{\theta}=\left(\begin{array}{c}
\operatorname{Re} \varphi\left(r_{1} ; \theta\right) \\
\vdots \\
\operatorname{Re} \varphi\left(r_{q} ; \theta\right) \\
\operatorname{Im} \varphi\left(r_{1} ; \theta\right) \\
\vdots \\
\operatorname{Im} \varphi\left(r_{q} ; \theta\right)
\end{array}\right)_{2 q \times 1}
\end{gathered}
$$

Note que

$$
\frac{1}{n} \sum_{j=1}^{n} f\left(X_{j} ; \theta\right)=V_{n}-V_{\theta} .
$$

O estimador GMM é obtido por

$$
\widehat{\theta}_{n}^{*}=\arg \min _{\theta}\left(V_{n}-V_{\theta}\right)^{\prime} W_{n}\left(V_{n}-V_{\theta}\right),
$$

em que $W_{n}$ é uma seqüência de matrizes positivas e definidas que convergem quase certamente para uma matriz de constantes $W_{0}$ positiva e definida.

Se $W_{n}=I_{2 q}$ então o estimador é basicamente uma regressão não linear de $V_{n}$ em $V_{\theta}$, ou seja pode-se obter $\theta$ a partir do método dos mínimos quadrados. Mas como a matriz de covariância de $V_{n}$ não é diagonal o estimador obtido, embora consistente, não é eficiente no sentido GMM. Para isso, basta utilizar $W_{n}=\widehat{\Omega}^{-1}$, em que $\widehat{\Omega}$ é algum estimador consistente 
da matriz de covariâncias de $V_{n}$, dado por:

$$
\Omega=\left[\begin{array}{cc}
\Omega_{R R} & \Omega_{R I} \\
\Omega_{I R} & \Omega_{I I}
\end{array}\right] .
$$

Para o caso i.i.d. os elementos das partições associados com $r_{j}$ e $r_{k}$ são dados em Knight e Satchell (1997), com base nos resultados de Feuerverger e McDunnough (1981b), por

$$
\begin{aligned}
\left(\Omega_{R R}\right)_{j k} & =\frac{1}{2}\left\{\operatorname{Re} \varphi\left(r_{j}+r_{k}\right)+\operatorname{Re} \varphi\left(r_{j}-r_{k}\right)\right\}-\operatorname{Re} \varphi\left(r_{j}\right) \operatorname{Re} \varphi\left(r_{k}\right), \\
\left(\Omega_{R I}\right)_{j k} & =\frac{1}{2}\left\{\operatorname{Im} \varphi\left(r_{j}+r_{k}\right)-\operatorname{Im} \varphi\left(r_{j}-r_{k}\right)\right\}-\operatorname{Re} \varphi\left(r_{j}\right) \operatorname{Im} \varphi\left(r_{k}\right), \\
\left(\Omega_{I R}\right)_{j k} & =\frac{1}{2}\left\{\operatorname{Im} \varphi\left(r_{j}+r_{k}\right)+\operatorname{Im} \varphi\left(r_{j}-r_{k}\right)\right\}-\operatorname{Im} \varphi\left(r_{j}\right) \operatorname{Re} \varphi\left(r_{k}\right), \\
\left(\Omega_{I I}\right)_{j k} & =\frac{1}{2}\left\{-\operatorname{Re} \varphi\left(r_{j}+r_{k}\right)+\operatorname{Re} \varphi\left(r_{j}-r_{k}\right)\right\}-\operatorname{Im} \varphi\left(r_{j}\right) \operatorname{Re} \varphi\left(r_{k}\right) .
\end{aligned}
$$

Sob condições de regularidade apropriadas Feuerverger e McDunnough (1984) mostram que para o caso $W_{n}=\widehat{\Omega}^{-1}$ temos

$$
\sqrt{n}\left(\widehat{\theta}_{n}^{*}-\theta_{0}\right) \stackrel{d}{\longrightarrow} N\left(0,\left(\frac{\partial V_{\theta}\left(\theta_{0}\right)^{\prime}}{\partial \theta} W_{0} \frac{\partial V_{\theta}\left(\theta_{0}\right)}{\partial \theta}\right)^{-1}\right) .
$$

A dificuldade básica deste método refere-se a escolha do número de pontos $q$ e de sua particular malha. Schmidt (1982) sugere que, fixado um $q$, deve-se escolher uma malha de pontos que minimiza o determinante da matriz de covariâncias assintótica do estimador. Knight e Satchell (1997) por sua vez sugerem o seguinte procedimento:

(a) Defina $q$ e um conjunto arbitrário de pontos $r_{1}, \cdots, r_{q}$. Os autores afirmam que obtiveram bons resultados com $q$ igual a 2 ou 3 vezes o tamanho da amostra.

(b) Calcule a regressão não linear $V_{n}=V_{\theta}+u$. Com o estimador $\theta_{n}^{(1)}$ assim obtido calcule $\Omega_{n}^{(1)}$, um estimador consistente da matriz $\Omega$, utilizando $\theta=\theta_{n}^{(1)}$ e os $r_{j}$ 's escolhidos.

(c) Considere $\Sigma_{\theta}$ a matriz de covariâncias do estimador $\theta_{n}$ calculada em $\theta_{n}^{(1)}$ para um conjunto $r_{j}^{*}=\tau . r_{j}$. Obtenha $\tau^{*}$ de maneira a minimizar o determinante de $\Sigma_{\theta}$. Note que este procedimento de otimização é univariado $(\tau)$. Alternativamente pode-se também procurar obter todo o vetor $\left(r_{1}^{*}, \cdots, r_{q}^{*}\right)^{\prime}$ através do procedimento de minimização de $\Sigma_{\theta}$.

(d) Refaça o passo (b) utilizando $r_{j}^{*}=\tau^{*} \cdot r_{j}$ (ou o vetor $r^{*}$ obtido da otimização), obtendo assim novos estimadores $\theta_{n}^{(2)}$ e $\Omega_{n}^{(2)}$.

(e) Usando os $r_{j}^{*}$ 's e $\Omega_{n}^{(2)}$ calcule uma regressão de mínimos quadrados generalizados (GLS - Generalized Least Squares) e obtenha o estimador desejado $\widehat{\theta}_{n}$. 
Feuerverger e McDunnough (1981b) sugerem que se $q$ for suficientemente grande e a malha suficientemente fina, pode-se obter matriz de covariâncias arbitrariamente próxima do limite de Cramér-Rao (i.e., pode-se alcançar eficiência de máxima verossimilhança). Entretanto Carrasco e Florens (2002) afirmam que esta passagem para o limite pode ser problemática, e sugerem o emprego do seu procedimento GMM estendido para uma condição contínua de momentos, que passamos a descrever a seguir.

\subsection{Extensão do GMM para um contínuo de momentos}

Carrasco e Florens (2000b) estendem o método de estimação GMM para o caso de um contínuo de condições de momentos, usando para isso uma abordagem de espaços de Hilbert. A aplicação deste método para o problema de estimação usando a ECF é feita por Carrasco e Florens (2002).

Para apresentar os resultados de Carrasco e Florens (2002) suponhamos que $X_{1}, \cdots, X_{n}$ sejam realizações de uma mesma variável aleatória $X \in \mathbb{R}^{p}$ com função distribuição $F\left(x ; \theta_{0}\right)$, com parâmetro $\theta_{0} \in \mathbb{R}^{q}$. Seja $\pi$ uma função densidade de probabilidade em $\mathbb{R}^{p}$ e seja $L^{2}(\pi)$ o espaço de Hilbert das funções complexas de quadrado do módulo integrável:

$$
L^{2}(\pi)=\left\{f:\left.\mathbb{R}^{p} \rightarrow \mathbb{C}\left|\int\right| f(r)\right|^{2} \pi(r) d r<\infty\right\}
$$

Para $f$ e $g$ vetores $l \times 1$ de elementos em $L^{2}(\pi)$, vamos denotar o produto interno por:

$$
\langle f, g\rangle=\int f(r) \overline{g(r)}^{\prime} \pi(t) d r
$$

A norma de $g$ neste espaço é denotada por $\|g\|$, sendo obtida por:

$$
\|g\|^{2}=\int g(r) \overline{g(r)}^{\prime} \pi(r) d r
$$

Seja a condição de momento dada por:

$$
h\left(r, X_{j} ; \theta\right)=e^{i r^{\prime} X_{j}}-\varphi(r ; \theta)
$$

e seja

$$
h_{n}(r ; \theta)=\frac{1}{n} \sum_{j=1}^{n} h\left(r, X_{j} ; \theta\right)
$$

Seja agora $H=L^{2}(\pi)$ o espaço de Hilbert de referência. Seja $B$ um operador linear limitado definido em $H$, e seja $B_{n}$ uma seqüência de operadores lineares limitados em $H$, ou em um subespaço de $H$, que convirjam para $B$. O estimador GMM é definido como

$$
\widehat{\theta}_{n}=\arg \min _{\theta}\left\|B_{n} h_{n}(. ; \theta)\right\|
$$


e sob as condições listadas em Carrasco e Florens (2000b) é consistente e assintoticamente normal. Além disso, Carrasco e Florens (2002) mostram que, dentro da classes dos operadores $B$, existe um operador $K^{-1 / 2}$ que fornece estimadores de mínima variância, em que $K: f \in$ $H \rightarrow g \in H$ é o operador de covariância associado com $h\left(r, X_{j} ; \theta\right)$ :

$$
K f(r)=g(s)=\int k(s, r) f(r) \pi(r) d r
$$

em que

$$
k(s, r)=E_{\theta_{0}}[h(s, X ; \theta) \overline{h(r, X ; \theta)}] .
$$

Para o caso do estimador usando a ECF, Carrasco e Florens (2002) propõem o núcleo

$$
k(s, r)=\varphi\left(s-r ; \theta_{0}\right)-\varphi\left(s ; \theta_{0}\right) \cdot \varphi\left(-r ; \theta_{0}\right) .
$$

O operador $K$ é auto-adjunto, isto é, $\langle K f, g\rangle=\langle f, K g\rangle$, já que $k(s, r)=\overline{k(r, s)}$, e daí decorre que, segundo Carrasco e Florens (2000b), $K$ tem um número (infinito) contável de auto-valores positivos decrescendo para zero; como conseqüência, sua inversa não é limitada Assim, $K^{-1 / 2}$ não existe em todo o espaço $H$, mas apenas em um subconjunto chamado núcleo reprodutivo do espaço de Hilbert (RKHS - Reproducing Kernel Hilbert Space) associado com $K$, denotado $\Re(K)$.

A escolha do estimador ótimo está associada com a obtenção do operador inverso do operador $K$, o que equivale a encontrar $\phi$, solução de uma equação integral de Fredholm de primeiro tipo ${ }^{2}$ :

$$
K \phi=f
$$

Este problema é tipicamente mal colocado: em particular, a solução $\phi$ de (3.25) é instável à pequenas mudanças em $f$. Para estabilizar a solução os autores utilizam a aproximação de Tikhonov, que consiste em introduzir um parâmetro de regularização $\alpha_{n}$ que vai para zero quando $n$ vai para o infinito:

$$
\left(K^{2}+\alpha_{n} I\right) \phi_{n}=K f
$$

Através da solução da equação acima os autores obtém o seguinte estimador consistente para $K^{-1 / 2}$ :

$$
\left(\widehat{K}_{n}^{\alpha_{n}}\right)^{-1 / 2} f=\sum_{j=1}^{n} \frac{\sqrt{\widehat{\mu}_{j}}}{\sqrt{\widehat{\mu}_{j}^{2}+\alpha_{n}}}\left\langle f, \phi_{j}\right\rangle \phi_{j},
$$

onde $\mu_{j}$ e $\phi_{j}$ são estimadores dos autovalores e das autofunções associadas a $K$, isto é, satisfazem a equação:

$$
K \phi=\mu \phi
$$

\footnotetext{
${ }^{2}$ Segundo Krevszig (1978, pg. 319), uma equação da forma

$$
\int_{a}^{b} k(t, \tau) x(\tau) d \tau=v(t)
$$

é dita equação de Fredholm do primeiro tipo. Aqui $[a, b]$ é um dado intervalo e $x($.$) é uma função desconhecida$ em $[a, b]$.
} 
Segundo os autores os autovalores $\mu_{j}$ podem ser obtidos diretamente a partir dos autovalores da matriz $M$, cujos elementos $m_{i j}$ são dados por:

$$
m_{i j}=\frac{1}{n} \int \overline{h\left(r, X_{i} ; \theta_{n}^{(1)}\right)} h\left(r, X_{j} ; \theta_{n}^{(1)}\right) \pi(r) d r
$$

sendo $\beta_{j}=\left(\beta_{j 1}, \cdots, \beta_{j n}\right)^{\prime}$ o $j$-ésimo autovetor, $\mu_{j}$ o $j$-ésimo autovalor de $M, j=1, \cdots, n$, e $\theta_{n}^{(1)}$ um estimador consistente de $\theta_{0}$.

As autofunções de $K_{n}$ por sua vez podem ser estimadas por:

$$
\widehat{\phi}_{j}(r)=h^{*}(r) \beta_{j}
$$

em que

$$
h^{*}(r)=\left(\begin{array}{c}
h\left(r, X_{1} ; \theta_{n}^{(1)}\right) \\
\vdots \\
h\left(r, X_{n} ; \theta_{n}^{(1)}\right)
\end{array}\right) .
$$

O estimador GMM ótimo de Carrasco e Florens (2002) é dado, finalmente, por:

$$
\widehat{\theta}_{n}=\arg \min _{\theta \in \Theta} \sum_{j=1}^{n} \frac{\widehat{\mu}_{j}}{\widehat{\mu}_{j}^{2}+\alpha_{n}}\left|\left\langle h_{n}(\theta), \widehat{\phi}_{j}\right\rangle\right|^{2}
$$

Carrasco e Florens (2002) utilizam o seguinte conjunto de hipóteses para obter o resultado de convergência assintótica do seu estimador:

(a) Os dados observados $\left\{x_{1}, \cdots, x_{n}\right\}$ são realizações i.i.d. de $X$. A variável aleatória $X$ assume valores em $\mathbb{R}^{p}$ e tem uma função densidade de probabilidade $f(x ; \theta)$ com $\theta \in \Theta \subset \mathbb{R}^{q}$ e $\Theta$ compacto.

(b) $\pi$ é uma função densidade de probabilidade absolutamente contínua com relação à medida de Lebesgue em $\mathbb{R}^{p} . \pi(x)>0$ para todo $x \in \mathbb{R}^{p}$.

(c) A equação

$$
E_{\theta_{0}}\left[e^{i r^{\prime} X}-\varphi(r ; \theta)\right]=0 \text { a.s. }
$$

tem uma solução única $\theta_{0}$ que é ponto interior de $\Theta$.

(d) Valem as seguintes condições:

a) $f(x ; \theta)$ é contínuamente diferenciável com respeito a $\theta=\left(\theta_{1}, \cdots, \theta_{q}\right)^{\prime}$ em $\Theta$,

b) $\int \sup _{\theta \in \Theta}\left\|\frac{\partial f(x ; \theta)}{\partial \theta}\right\| d x<\infty$,

c) $I_{\theta_{0}}=\left.E_{\theta}\left[\left(\frac{\partial \ln f(x ; \theta)}{\partial \theta}\right)\left(\frac{\partial \ln f(x ; \theta)}{\partial \theta}\right)^{\prime}\right]\right|_{\theta=\theta_{0}}$ é positiva definida.

Com estas hipóteses os autores obtêm o seguinte resultado de convergência. 
Proposição 3.1 (Prop. 4.3, Carrasco e Florens (2002)) Seja $\widehat{\theta}_{n}$ o estimador definido por (3.32). Sob hipóteses (a) a (d), temos

$$
\sqrt{n}\left(\widehat{\theta}_{n}-\theta_{0}\right) \stackrel{d}{\longrightarrow} N\left(0, I_{\theta_{0}}^{-1}\right)
$$

onde

$$
I_{\theta_{0}}=\left.E_{\theta}\left[\left(\frac{\partial \ln f(x ; \theta)}{\partial \theta}\right)\left(\frac{\partial \ln f(x ; \theta)}{\partial \theta}\right)^{\prime}\right]\right|_{\theta=\theta_{0}}
$$

à medida que n e no $\alpha_{n}^{2}$ vão para o infinito e $\alpha_{n}$ vai para zero.

Ou seja, o estimador proposto alcança o limite de Cramér-Rao, e, portanto, é eficiente (no sentido de máxima verossimilhança). Observe, entretanto, que o método proposto exige a obtenção dos autovalores e autovetores da matriz $M$, que é potencialmente de ordem muito grande (da ordem de $T \times T$, em que $T$ é o tamanho da amostra).

\subsection{Sumário}

Neste capítulo revisamos alguns estimadores propostos na literatura que se utilizam da ECF:

- Estimador CECF de Knight e Yu (2002);

- Estimador de método dos momentos (DECF);

- Extensão do GMM para contínuo de momentos, de Carrasco e Florens (2002)

Também foram apresentados os resultados de convergência assintótica para os estimadores acima. Convém ressaltar que os resultados obtidos valem apenas para séries estacionárias, para o caso dos dois primeiros estimadores (CECF e DECF), e apenas para séries i.i.d. para o caso do terceiro estimador (extensão do GMM para contínuo de momentos).

Neste trabalho decidimos utilizar os estimadores CECF e DECF para o problema da duração condicional estocástica. No capítulo seguinte vamos formular o problema de interesse, e vamos procurar obter os elementos necessários para emprego dos métodos CECF e DECF. 


\section{Capítulo 4}

\section{O modelo de Duração Condicional Estocástica (SCD)}

Atualmente nos mercados financeiros é possível coletar-se dados de alta-freqüência , isto é, dados relativos a cada operação (negócio) efetuada ou coletados a intervalos curtos de tempo (5 min, por exemplo). Esta abundância de dados permite o estudo de fenômenos relacionados a micro-estrutura de mercado, como, por exemplo, o impacto das transações nos preços (o chamado impacto de mercado) e a volatilidade intra-diária (por exemplo, através da medida chamada volatilidade realizada). Em particular, pretendemos trabalhar com os chamados modelos de duração, que tem como objetivo a estimação da distribuição de probabilidade do intervalo de tempo entre eventos ${ }^{1}$.

Em Finanças os eventos que estamos interessados podem ser, por exemplo, o intervalo de tempo entre o fechamento de transações (trade duration) ou o intervalo de mudança de preço do ativo (price duration). A distribuição da duração está intimamente relacionada ao conceito de "liquidez": ativos mais "líquidos" usualmente possuem intervalos de negociação menores do que ativos menos líquidos. Outra medida da "liquidez" é o spread entre o preço de compra (bid) e o de venda $(a s k)$. Evidentemente, o intervalo de tempo para efetuar um negócio depende das condições de oferta e demanda do mercado, mas, fixadas as outras condições, quanto menor o preço de venda ou maior o preço de compra, mas "rápido" a negociação ocorrerá. A situação parece mais complicada em mercados organizados com a presença de especialistas $^{2}$, que não necessariamente desejam carregar uma posição no ativo. Isto é, ao abrir uma posição indesejada (digamos que ele tenha comprado o ativo), o especialista corre o risco da mudança de preço do ativo até que consiga se desfazer da posição (neste caso, vendê-la). Assim, parece natural que os especialistas estabeleçam spreads de compra e venda de maneira a remunerar o risco da posição, o que está intimamente ligado a duração que a

\footnotetext{
${ }^{1}$ Outro tipo de modelo de duração é aquele associado com cadeias de Markov, e que procura estimar o tempo que o indivíduo permanece em cada estado da cadeia. Para mais detalhes veja, por exemplo, Amemiva (1985).

${ }^{2}$ Especialista são figuras em determinados mercados (e.g., a bolsa de valores de New York - NYSE) que mantém um mercado em um ou mais ativos listados. A função do especialista é oferecer spreads de compra e venda do ativo sob sua responsabilidade (a NYSE, por exemplo, designa cada ação listada a um especialista para a função de market-making).
} 
posição permanece em seu estoque ${ }^{3}$.

Neste capítulo vamos rever brevemente dois modelos propostos para estudar a duração. Na seção seguinte vamos especificar o modelo de duração condicional autoregressiva (ACD) proposto por Engle e Russell (1998). A seguir vamos especificar o modelo de duração condicional estocástica (SCD) proposto por Bauwens e Veredas (2004), que é o nosso objeto de estudo. Nas seções posteriores, vamos obter algumas de suas propriedades que nos serão úteis no processo de estimação utilizando a ECF.

\subsection{O modelo de duração condicional autorregressiva (ACD)}

Para estudar o fenômeno da duração entre negócios, Engle e Russell (1998) propõem o modelo de duração condicional autorregressiva (ACD - Autoregressive Conditional Duration). No modelo $\operatorname{ACD}(p, q)$, a duração $X_{j}$, calculada pela diferença entre os instantes de negociação $t_{j}$ e $t_{j-1}$, é dada por

$$
X_{j}=\psi_{j} \xi_{j}
$$

em que $\left\{\xi_{j}\right\}$ é uma seqüência de variáveis aleatórias não negativas identicamente distribuídas tais que $E\left[\xi_{j}\right]=1$, e $\psi_{j}$ é especificado como um processo autoregressivo

$$
\psi_{j}=\delta+\sum_{k=1}^{p} \phi_{k} X_{j-k}+\sum_{k=1}^{q} \delta_{k} \psi_{j-k} .
$$

Engle e Russell (1998) utilizam para $\varepsilon_{j}$ a distribuição de Weibull com média 1, dada por

$$
f\left(\xi_{j}\right)=\left\{\begin{array}{c}
\lambda\left[\Gamma\left(1+\frac{1}{\lambda}\right)\right]^{\lambda} \xi_{j}^{\lambda-1} e^{-\left[\Gamma\left(1+\frac{1}{\lambda}\right) \xi_{j}\right]^{\lambda}}, \text { se } \xi_{j} \geq 0 \\
0, \text { se } \xi_{j}<0
\end{array}\right.
$$

Um caso particular ocorre para $\lambda=1$, quando a distribuição em (4.3) reduz-se à distribuição exponencial.

Nesta especificação $\psi_{j}$ é o valor esperado da duração, condicional na informação $I_{j-1}$ disponível até $j-1$, i.e., $\psi_{j}=E\left[X_{j} \mid I_{j-1}\right]$. Desta forma a evolução do valor esperado da duração neste modelo é determinada pela variável latente $\psi_{j}$. Note, também, que a especificação do modelo $\operatorname{ACD}(p, q)$ é análoga a de um modelo $\operatorname{GARCH}(p, q)$; sendo assim, o modelo é facilmente estimável através do método da máxima verossimilhança (veja Engle e Russell (1998) ou Tsav (2002) para mais detalhes).

Bauwens e Veredas (2004) fazem uma revisão bibliográfica das possíveis extensões do modelo ACD. Por exemplo, a fim de evitar restrições nos parâmetros que garantam que $\psi_{j}$ seja positivo, pode-se utilizar $e^{\psi_{j}}$ na eq. (4.1), de forma que $X_{j}=e^{\psi_{j}} \xi_{j}$. Outra revisão bastante completa do modelo ACD e suas extensões pode ser encontrada em Pacurar (2006).

\footnotetext{
${ }^{3}$ Mais detalhes sobre a organização e o funcionamento dos mercados podem ser encontrados em Bodie et al. (2002), sob o aspecto descritivo do seu funcionamento, e em Madhavan (2000), sob o ponto de vista de modelagem teórica e empírica. Também Engle e Russell (1998) examinam alguns aspectos da microestrutura do mercado relacionados com a duração entre negócios.
} 


\subsection{O modelo de duração condicional estocástica (SCD)}

Bauwens e Veredas (2004) propõem o modelo de duração condicional estocástica (SCD Stochastic Conditional Duration) no qual a variável latente $\psi_{j}$, que determina as durações como no modelo ACD, passa a ser uma variável aleatória seguindo um processo autoregressivo $\operatorname{AR}(1)^{4}$, de maneira que o modelo possa ser escrito por

$$
\begin{aligned}
X_{j} & =e^{\psi_{j}} \xi_{j}, \\
\psi_{j} & =\delta+\phi \psi_{j-1}+\sigma_{\eta} \eta_{j}, \quad|\phi|<1,
\end{aligned}
$$

com as seguintes hipóteses distribucionais

$$
\begin{aligned}
\eta_{j} \mid I_{j-1} \sim & N(0,1), \\
\xi_{j} \mid I_{j-1} \sim & \text { alguma distribuição com suporte positivo } \\
& \eta_{k} \text { independente de } \xi_{j} \mid I_{j-1}, \forall j, k .
\end{aligned}
$$

Em particular, Bauwens e Veredas (2004) assumem as seguintes distribuições para $\xi_{j}$ :

- distribuição Weibull padrão, $\xi_{j} \sim W(\lambda, 1)$, com densidade

$$
f\left(\xi_{j}\right)=\left\{\begin{array}{c}
\lambda \xi_{j}^{\lambda-1} e^{-\xi_{j}^{\lambda}}, \text { se } \xi_{j} \geq 0 \\
0, \text { se } \xi_{j}<0
\end{array},\right.
$$

e com momentos não centrados dados por

$$
E\left(\xi_{j}^{n}\right)=\Gamma\left(1+\frac{n}{\lambda}\right)
$$

- distribuição gama padrão, $\xi_{j} \sim G(\lambda, 1)$, com densidade

$$
f\left(\xi_{j}\right)=\left\{\begin{array}{c}
\frac{1}{\Gamma(\lambda)} \xi_{j}^{\lambda-1} e^{-\xi_{j}}, \text { se } \xi_{j} \geq 0 \\
0, \text { se } \xi_{j}<0
\end{array}\right.
$$

e com momentos não centrados dados por

$$
E\left(\xi_{j}^{n}\right)=\frac{\Gamma(\lambda+n)}{\Gamma(\lambda)} .
$$

Note que ambas as distribuições propostas reduzem-se à distribuição exponencial de média 1 quando $\lambda=1$.

Comparando (4.4)-(4.5) com (4.1)-(4.2) podemos notar que a principal diferença do modelo SCD em relação ao modelo ACD é a presença de uma choque aleatório adicional $\eta_{j}$ na variável latente $\psi_{j}$.

\footnotetext{
${ }^{4} \mathrm{O}$ processo para $\psi_{j}$ poderia seguir um processo de ordem maior, $\operatorname{AR}(p)$, mas para manter a comparabilidade com os artigos originais, vamos manter $\mathrm{AR}(1)$.
} 
Da mesma forma que o modelo ACD é análogo a um modelo GARCH, o modelo SCD é análogo ao modelo de volatilidade estocástica. Em particular, Bauwens e Veredas (2004) discutem diversos métodos de estimação de volatilidade estocástica que podem ser utilizados para estimar o modelo SCD. Os autores também obtiveram os dois primeiros momentos e a função de autocorrelação das durações, utilizando a notação $\Psi_{j}=e^{\psi_{j}}$ nos seguintes teoremas:

Teorema 4.1 (Bauwens e Veredas (2004), Teor. 1) As durações e as variáveis latentes do modelo (4.4)-4.8) têm os seguintes momentos:

$$
\begin{aligned}
E\left[\Psi_{j}\right] & =e^{\frac{\delta}{1-\phi}+\frac{\sigma_{\eta}^{2}}{2\left(1-\phi^{2}\right)}}, \\
E\left[X_{j}\right] & =E\left[\xi_{j}\right] \cdot E\left[\Psi_{j}\right], \\
\operatorname{Var}\left[\Psi_{j}\right] & =\left(E\left[\Psi_{j}\right]\right)^{2}\left(e^{\frac{\sigma_{\eta}^{2}}{1-\phi^{2}}}-1\right), \\
\operatorname{Var}\left[X_{j}\right] & =\left(E\left[X_{j}\right]\right)^{2}\left(\kappa e^{\frac{\sigma_{\eta}^{2}}{1-\phi^{2}}}-1\right),
\end{aligned}
$$

em que

$$
\kappa=\frac{E\left[\xi_{j}^{2}\right]}{\left(E\left[\xi_{j}\right]\right)^{2}} .
$$

Prova. Ver Bauwens e Veredas (2004), Teor.1, pg. 386.

Teorema 4.2 (Bauwens e Veredas (2004), Teor. 2) A função de autocorrelação (ACF) do modelo 4.4)-(4.8) é dada por

$$
\rho_{s}=\frac{e^{\frac{\sigma_{\eta}^{2} \phi^{s}}{1-\phi^{s}}-1}}{\kappa e^{\frac{\sigma_{\eta}^{2}}{1-\phi^{s}}-1}} .
$$

Prova. Ver Bauwens e Veredas (2004), Teor.2, pg. 386.

Antes de prosseguirmos note que, como a variável $\psi_{j}$ segue um processo AR(1), pode ser representada como estabelecido na proposição abaixo.

Proposição 4.1 (Representação da variável latente $\psi_{j}$ ) A seqüência de variáveis latentes $\left\{\psi_{j}\right\}$ definidas em (4.4)- 4.8) constitui um processo (fracamente) estacionário, com a seguinte representação:

$$
\psi_{j}=\frac{\delta}{1-\phi}+\sigma_{\eta} \sum_{l=0}^{\infty} \phi^{l} \eta_{j-l} .
$$

Além disso, $\psi_{j}$ tem a seguinte distribuição marginal:

$$
\psi_{j} \sim N\left(\frac{\delta}{1-\phi}, \frac{\sigma_{\eta}^{2}}{1-\phi^{2}}\right)
$$


Prova. A demonstração desta proposição encontra-se no apêndice $\square$.

Esta representação é útil para obtenção da função característica de $\psi_{j}$. Para isso, lembremos que os $\eta_{j}$ são identicamente e independentemente distribuídos como $N(0,1)$, cuja função característica é dada por:

$$
\varphi_{\eta}(t)=\exp \left(-\frac{t^{2}}{2}\right)
$$

Podemos agora obter a função característica de $\psi_{j}$ a partir de (4.13), através da seguinte proposição:

Proposição 4.2 (Função característica da variável latente $\psi_{j}$ ) A função característica da variável latente definida em (4.5) do modelo (4.4)-(4.8) é dada por:

$$
\varphi_{\psi}(t)=\exp \left(\frac{i t \delta}{1-\phi}-\frac{\sigma_{\eta}^{2} t^{2}}{2\left(1-\phi^{2}\right)}\right) .
$$

Prova. A demonstração desta proposição encontra-se no apêndice $\square$.

A seguir vamos formular o modelo SCD de interesse, obter algumas propriedades das distribuições envolvidas, e obter a verossimilhança amostral, ilustrando assim a dificuldade que este modelo possui para estimação de seus parâmetros.

\subsection{Formulação do modelo de interesse}

Vamos definir as seguintes variáveis: $\tau_{j}$ instante da ocorrência da $j$-ésima transação, $X_{j}=$ $\tau_{j}-\tau_{j-1}$ a $j$-ésima duração entre transações sucessivas e $X=\left(X_{1}, X_{2}, \cdots, X_{T}\right)^{\prime}$ vetor $T \times 1$ das durações, e $\tau_{0}$ o instante de abertura do mercado. Vamos assumir que $X_{j}>0$, ou seja, que não possam ocorrer duas transações no mesmo instante. O nosso modelo de interesse é dado por $^{5}$ :

$$
\begin{aligned}
& X_{j}=e^{\psi_{j}} \xi_{j}, \\
& \psi_{j}=\delta+\phi \psi_{j-1}+\sigma_{\eta} \eta_{j},
\end{aligned}
$$

em que $|\phi|<1, \sigma_{\eta}<\infty$, e com as seguintes hipóteses distribucionais

$$
\begin{aligned}
\eta_{j} \mid I_{j-1} \sim & N(0,1), \\
& \eta_{k} \text { independente de } \xi_{j} \mid I_{j-1}, \forall j, k,
\end{aligned}
$$

\footnotetext{
${ }^{5} \mathrm{Na}$ especificação de Strickland et al. (2006) aparece uma variável exógena $W_{j-1}$ de dimensão $k \times 1$ da seguinte forma: $\psi_{j}=\delta^{\prime} W_{j-1}+\phi \psi_{j-1}+\sigma_{\eta} \eta_{j}$. Entretanto, a presença desta variável torna o processo $\psi_{j}$ não estacionário. Assim, não pretendemos tratar este problema aqui neste trabalho.
} 
e o erro $\xi_{j}$ com as distribuições dadas por (4.9) ou por (4.11), mas "padronizadas" de forma que $E\left[\xi_{j}\right]=1$, de maneira a permitir a comparação dos $\psi_{j}$ obtidos com as diversas distribuiçõos ${ }^{6}$. Como as distribuições propostas em (4.9) e (4.11) tem valor esperado diferente de 1 precisamos "padronizar" as distribuições utilizando a seguinte proposição:

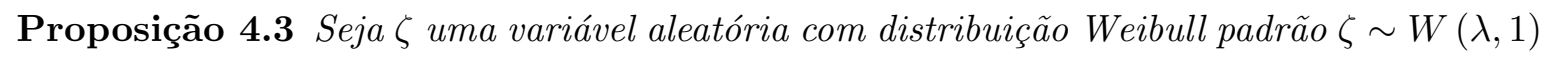
ou com distribuição gama padrão $\zeta \sim G(\lambda, 1)$, e seja $\xi$ a variável aleatória "padronizada", definida por

$$
\xi=\frac{\zeta}{E[\zeta]}
$$

Caso $\zeta$ tenha densidade Weibull $\zeta \sim W(\lambda, 1)$, então $\xi$ tem densidade dada por

$$
f_{\xi}(\xi)=\left\{\begin{array}{c}
\lambda\left[\Gamma\left(1+\lambda^{-1}\right)\right]^{\lambda} \xi^{\lambda-1} e^{-\left[\Gamma\left(1+\lambda^{-1}\right) \xi\right]^{\lambda}}, \text { se } \xi \geq 0 \\
0, \text { se } \xi<0
\end{array}\right.
$$

Caso $\zeta$ tenha densidade gama $\zeta \sim G(\lambda, 1)$, então $\xi$ tem densidade dada por

$$
f_{\xi}(\xi)=\left\{\begin{array}{c}
\frac{\lambda^{\lambda}}{\Gamma(\lambda)} \xi^{\lambda-1} e^{-\lambda \xi}, \text { se } \xi \geq 0 \\
0, \text { se } \xi<0
\end{array}\right.
$$

Prova. A demonstração desta proposição encontra-se no apêndice C.

Como as durações $X_{j}$, condicionais em variáveis latentes estocásticas $\psi_{j}$, são independentes entre si, podemos obter a probabilidade condicional conjunta do vetor $X$ por:

$$
p(x \mid \psi)=\prod_{j=1}^{T} p\left(x_{j} \mid \psi_{j}\right) .
$$

Assumindo as densidades (4.21) ou (4.22) para $\xi_{j}$ podemos obter a distribuição de probabilidade da duração $X_{j}$ condicional em $\psi_{j}$, através da seguinte proposição:

Proposição 4.4 (Densidade condicional de $X_{j}$ dado $\psi_{j}$ ) Seja $X_{j}=e^{\psi_{j}} \xi_{j}$, sendo a distribuição de $\xi_{j}$ dada por 4.21) ou 4.22). Neste caso, a densidade de $X_{j}$ condicional em $\psi_{j}$ é dada para o caso Weibull por:

$$
f\left(x_{j} \mid \psi_{j}\right)=\left\{\begin{array}{c}
\lambda\left[\frac{\Gamma\left(1+\lambda^{-1}\right)}{e^{\psi_{j}}}\right]^{\lambda} x_{j}^{\lambda-1} e^{-\left[\frac{\Gamma\left(1+\lambda^{-1}\right)}{e^{\psi_{j}}} x_{j}\right]^{\lambda}}, \text { se } x_{j} \geq 0 \\
0, \text { se } x_{j}<0
\end{array}\right.
$$

e para o caso da distribuição gama por

$$
f\left(x_{j} \mid \psi_{j}\right)=\left\{\begin{array}{c}
\frac{1}{\Gamma(\lambda)}\left(\frac{\lambda}{e^{\psi_{j}}}\right)^{\lambda} x_{j}^{\lambda-1} e^{-\frac{\lambda}{e^{\psi_{j}}} x_{j}}, \text { se } x_{j} \geq 0 \\
0, \text { se } x_{j}<0
\end{array}\right.
$$

\footnotetext{
${ }^{6}$ Naturalmente, outras distribuições com suporte positivo também poderiam ser utilizadas, como, por exemplo, a distribuição exponencial generalizada (Gupta e Kundu, 2000).
} 
Caso $\lambda=1$ em qualquer das distribuições acima, obtemos o caso da distribuição exponencial

$$
f\left(x_{j} \mid \psi_{j}\right)=\left\{\begin{array}{c}
e^{-x_{j} e^{-\psi_{j}}} e^{-\psi_{j}}, \text { se } x_{j} \geq 0 \\
0, \text { se } x_{j}<0
\end{array} .\right.
$$

Prova. A demonstração desta proposição encontra-se no apêndice $\square$.

Proposição 4.5 (Distribuição condicional de $X_{j}$ dado $\psi_{j}$ ) Seja $X_{j}=e^{\psi_{j}} \xi_{j}$, sendo $a$ distribuição de $\xi_{j}$ dada por (4.21) ou (4.22). Neste caso, a função distribuição de probabilidade de $X_{j}$ condicional em $\psi_{j}$ é dada para o caso Weibull por:

$$
F_{X_{j} \mid \psi_{j}}\left(x_{j}\right)=\left\{\begin{array}{c}
1-e^{-\left[\frac{\Gamma\left(1+\lambda^{-1}\right)}{e^{\psi_{j}}} x_{j}\right]^{\lambda}}, \text { se } x_{j} \geq 0 \\
0, \text { se } x_{j}<0
\end{array}\right.
$$

e para o caso da distribuição gama por

$$
F_{X_{j} \mid \psi_{j}}\left(x_{j}\right)=\left\{\begin{array}{c}
\frac{\Gamma_{\lambda x_{j} / c}(\lambda)}{\Gamma(\lambda)}, \text { se } x_{j} \geq 0 \\
0, \text { se } x_{j}<0
\end{array}\right.
$$

em que $\Gamma_{\lambda x_{j} / c}(\lambda)$ é a função gama incompleta dada por

$$
\Gamma_{\lambda x_{j} / c}(\lambda)=\int_{0}^{\lambda x_{j} / c} y^{\lambda-1} e^{-y} d y
$$

$e$

$$
c=\frac{\Gamma\left(1+\lambda^{-1}\right)}{e^{\psi^{j}}} .
$$

Caso $\lambda=1$ em qualquer das distribuições acima, obtemos o caso da distribuição exponencial

$$
F_{X_{j} \mid \psi_{j}}\left(x_{j}\right)=\left\{\begin{array}{c}
1-e^{-x_{j} e^{-\psi_{j}}} e^{-\psi_{j}}, \text { se } x_{j} \geq 0 \\
0, \text { se } x_{j}<0
\end{array} .\right.
$$

Prova. A demonstração desta proposição encontra-se no apêndice $\square$.

As distribuições condicionais obtidas acima coincidem com as distribuições propostas em Strickland et al. $(2006)^{7}$.

${ }^{7}$ Para facilitar a comparação reescrevemos abaixo as equações como utilizadas por Strickland et al. (2006):

- Exponencial:

$p\left(x_{j} \mid \psi_{j}\right)=\exp \left\{-\psi_{j}\right\} \exp \left\{-x_{j} \exp \left(-\psi_{j}\right)\right\}$

- Weibull:

$p\left(x_{j} \mid \psi_{j}\right)=\lambda\left(\frac{\Gamma\left(1+\lambda^{-1}\right)}{\exp \left(\psi_{j}\right)}\right)^{\lambda} x_{j}^{\lambda-1} \exp \left\{-\left[\frac{\Gamma\left(1+\lambda^{-1}\right)}{\exp \left(\psi_{j}\right)} x_{j}\right]^{\lambda}\right\}$

- Gama:

$$
p\left(x_{j} \mid \psi_{j}\right)=\frac{1}{\Gamma(\lambda)}\left(\frac{\lambda}{\exp \left(\psi_{j}\right)}\right)^{\lambda} x_{j}^{\lambda-1} \exp \left\{-\frac{\lambda}{\exp \left(\psi_{j}\right)} x_{j}\right\}
$$


As distribuições (4.24) a (4.26) mostram que a relação entre $X_{j}$ e $\psi_{j}$ é não linear e bastante complicada. Para facilitar o tratamento, ao invés de trabalharmos diretamente com as durações vamos trabalhar com o seu logaritmo:

$$
Y_{j}=\ln X_{j}
$$

Neste caso o modelo (4.4)-(4.5) fica

$$
\begin{aligned}
Y_{j} & =\psi_{j}+\varepsilon_{j}, \\
\psi_{j} & =\delta+\phi \psi_{j-1}+\sigma_{\eta} \eta_{j}, \quad|\phi|<1,
\end{aligned}
$$

onde

$$
\varepsilon_{j}=\ln \xi_{j} .
$$

As distribuições de $Y_{j}$ e de $\varepsilon_{j}$ podem ser facilmente obtidas através do método do jacobiano. A seguir vamos obter as distribuições de probabilidade condicionais de $Y_{j}$ resultantes dessa transformação através da seguinte proposição:

Proposição 4.6 (Densidade condicional de $Y_{j}$ dado $\psi_{j}$ ) Seja $Y_{j}=\ln X_{j}$, tendo $X_{j}$ as distribuições condicionais estabelecidas na prop. 4.4. Neste caso, a densidade de $Y_{j}$ condicional em $\psi_{j}$ é dada para o caso exponencial por:

$$
f\left(y_{j} \mid \psi_{j}\right)=e^{y_{j}-\psi_{j}-e^{y_{j}-\psi_{j}}}
$$

Para o caso da distribuição Weibull temos

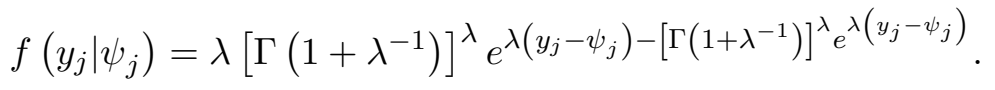

E para o caso da distribuição gama

$$
f\left(y_{j} \mid \psi_{j}\right)=\frac{\lambda^{\lambda}}{\Gamma(\lambda)} e^{\lambda\left(y_{j}-\psi_{j}\right)-\lambda e^{y_{j}-\psi_{j}}} .
$$

Prova. A demonstração desta proposição encontra-se no apêndice $\square$.

Os log-erros $\varepsilon_{j}$, por sua vez, são distribuídos identicamente e independentemente de acordo com a seguinte proposição:

Proposição 4.7 (Densidade de $\varepsilon_{j}$ ) Seja $\varepsilon_{j}=\ln \xi_{j}$, tendo $\xi_{j}$ as distribuições condicionais estabelecidas na prop. 4.3. Neste caso, a função densidade de probabilidade de $\varepsilon_{j}$ é dada para o caso exponencial por

$$
f\left(\varepsilon_{j}\right)=e^{\varepsilon_{j}-e^{\varepsilon_{j}}},
$$

que chamaremos de log-exponencial. 
Para o caso da distribuição Weibull temos

$$
f\left(\varepsilon_{j}\right)=\lambda\left[\Gamma\left(1+\lambda^{-1}\right)\right]^{\lambda} e^{\lambda \varepsilon_{j}-\left[\Gamma\left(1+\lambda^{-1}\right)\right]^{\lambda} e^{\lambda \varepsilon_{j}},}
$$

que chamaremos de log-Weibull.

Para o caso da distribuição gama temos

$$
f\left(\varepsilon_{j}\right)=\frac{\lambda^{\lambda}}{\Gamma(\lambda)} e^{\lambda \varepsilon_{j}-\lambda e^{\varepsilon_{j}}},
$$

que chamaremos de log-gama ${ }^{8}$.

Prova. Observando as equações (4.35), (4.36) e (4.37) notamos que as distribuições são função da diferença entre $y_{j}$ e $\psi_{j}$, que por (4.32) é dada por

$$
\varepsilon_{j}=y_{j}-\psi_{j}
$$

Substituindo (4.41) nas equações (4.35), 4.36) e 4.37) obtemos o resultado desejado.

A distribuição log-Weibull é a distribuição de Gumbel ou valor extremo tipo I, a menos de uma translação e uma mudança de sinal da variável. A densidade desta distribuição é geralmente apresentada na seguinte forma:

$$
f(x)=\frac{1}{\beta} e^{-\left(\frac{x-\mu}{\beta}\right)-e^{-\left(\frac{x-\mu}{\beta}\right)} .}
$$

Tomando

$$
\begin{aligned}
\beta & =\frac{1}{\lambda} \\
\mu & =\ln \Gamma\left(1+\lambda^{-1}\right) \\
x & =-\varepsilon
\end{aligned}
$$

obtemos a densidade (4.39). A densidade de Gumbel padronizada é uma caso particular, tomando $\mu=0$ e $\beta=1 \mathrm{em} \mathrm{(4.42)}$. As figs. 4.1 e 4.2 ilustram as densidades das três distribuições para dois valores de $\lambda$.

\subsection{Funções características para as distribuições dos $\log$-erros $\varepsilon_{j}$}

Vamos chamar de "log-erro" a variável $\varepsilon_{j}=\ln \xi_{j}$. As funções características dos log-erros, para as distribuições dos erros $\xi_{j}$ dadas pela proposição (4.3), são apresentadas na proposição

Eleiber e Kotz (2003) denominam de "log-gama" a distribuição de $X=e^{Y}$, em que $Y$ tem distribuição gama, que é exatamente a operação inversa que fizemos na proposição. Elucidada esta possível fonte de confusão, vamos entretanto manter neste texto o nome "log-gama" para a distribuição explicitada na proposição. 


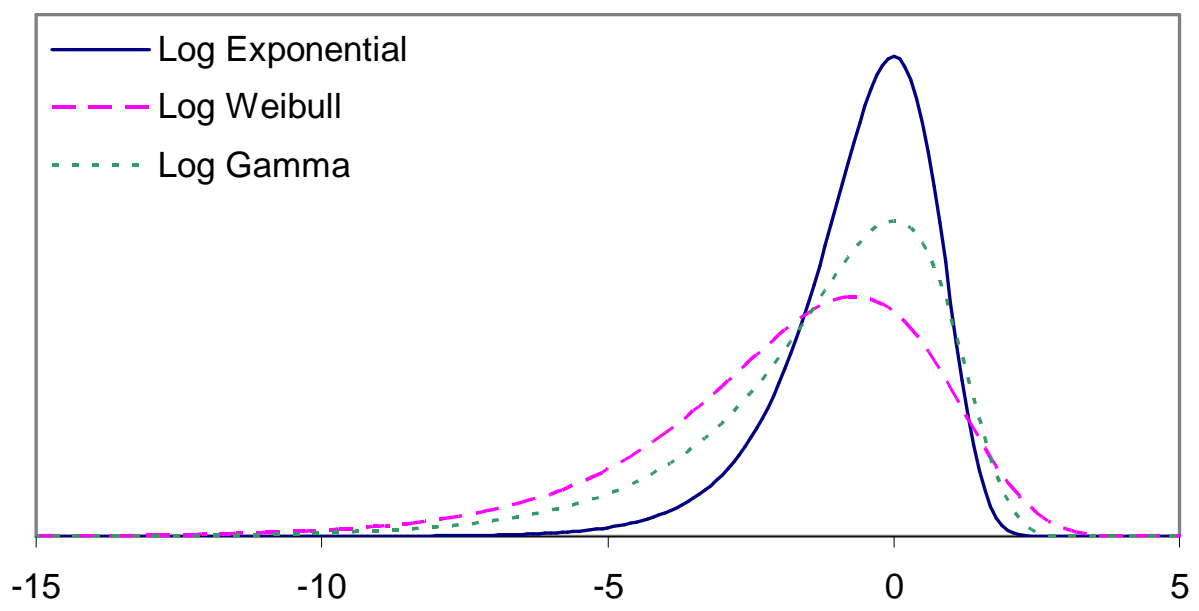

Figura 4.1: Funções densidade de probabilidade da log-exponencial (Gumbel), log-Weibull e log-gama, para $\lambda=0.5$.

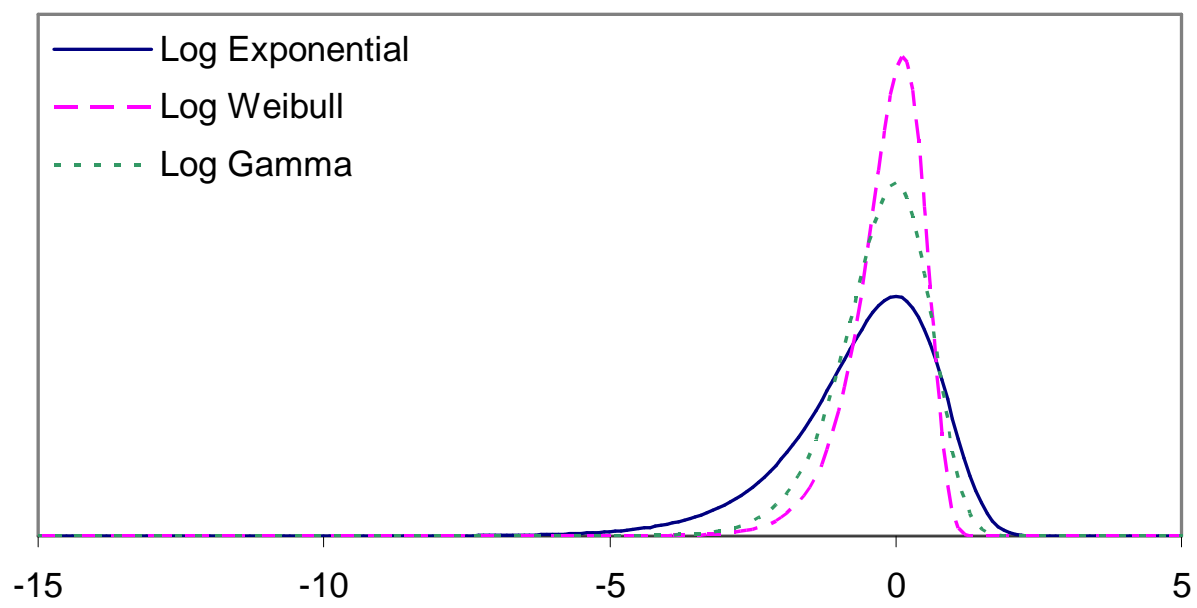

Figura 4.2: Funções densidade de probabilidade da log-exponencial (Gumbel), log-Weibull e log-gama, para $\lambda=2$.

abaixo.

Proposição 4.8 (Função característica de $\varepsilon_{j}$ ) Seja $\varepsilon_{j}$ uma variável aleatória com as distribuições estabelecidas na prop. 4.7. Neste caso, a função caracteristica de $\varepsilon_{j}$ é dada para o caso log-exponencial por

$$
\varphi_{\varepsilon}(t)=\Gamma(1+i t)
$$


Para o caso da distribuição log-Weibull temos

$$
\varphi_{\varepsilon}(t)=\frac{\Gamma\left(1+\frac{i t}{\lambda}\right)}{\left[\Gamma\left(1+\lambda^{-1}\right)\right]^{i t}} .
$$

E para o caso da distribuição log-gama

$$
\varphi_{\varepsilon}(t)=\frac{\Gamma(\lambda+i t)}{\lambda^{i t} \Gamma(\lambda)}
$$

Nas expressões acima temos que

$$
\Gamma(z)=\int_{0}^{\infty} \zeta^{z-1} e^{-\zeta} d \zeta
$$

representa a função gama com argumento complexo ${ }^{9}$.

Prova. A demonstração desta proposição encontra-se no apêndice $\square$.

\subsection{Cumulantes e momentos das distribuições dos log-erros $\varepsilon_{j}$}

Uma vez conhecida a função característica da distribuição de $\varepsilon_{j}$, podemos obter seus cumulantes a partir da função geratriz de cumulantes $G(t)$ :

$$
G(t)=\ln \varphi(t)=\sum_{r=1}^{\infty} \kappa_{r} \frac{(i t)^{r}}{r !}
$$

Expandindo $G(t)$ em uma série de Taylor em torno de $t=0$ obtemos:

$$
G(t)=\left.\sum_{r=1}^{\infty} \frac{\partial^{r} G}{\partial t^{t}}\right|_{t=0} \frac{t^{r}}{r !}
$$

Comparando (4.47) e (4.48) podemos obter o $r$-ésimo cumulante por:

$$
\kappa_{r}=\left.\frac{1}{i^{r}} \frac{\partial^{r} G}{\partial t^{t}}\right|_{t=0}=\frac{1}{i^{r}} G^{(r)}(0)
$$

Assim, para obter o $r$-ésimo cumulante precisamos da $r$-ésima derivada da função geratriz de cumulantes. A seguir vamos apresentar as funções geratrizes de cumulantes para $\varepsilon_{j}$ e suas respectivas derivadas através da seguinte proposição.

Proposição 4.9 (Função geratriz de cumulantes de $\varepsilon_{j}$ ) Seja $\varepsilon_{j}$ uma variável aleatória com as distribuiçôes condicionais estabelecidas na prop. 4.7. Neste caso, a função geratriz

\footnotetext{
${ }^{9}$ Para mais detalhes consulte, por exemplo, Abramowitz e Stegun (1972).
} 
de cumulantes de $\varepsilon_{j}$ é dada para o caso log-exponencial por

$$
G_{\varepsilon}(t)=\ln \Gamma(1+i t)
$$

com derivadas dadas por

$$
\begin{aligned}
G_{\varepsilon}^{\prime}(t) & =i \psi(1+i t) \\
G_{\varepsilon}^{(r)}(t) & =i^{r} \psi^{(r-1)}(1+i t), r>1 .
\end{aligned}
$$

Para o caso da distribuição log-Weibull temos

$$
G_{\varepsilon}(t)=\ln \Gamma\left(1+i t \lambda^{-1}\right)-i t \ln \Gamma\left(1+\lambda^{-1}\right),
$$

com derivadas dadas por

$$
\begin{aligned}
G_{\varepsilon}^{\prime}(t) & =\frac{i}{\lambda} \psi\left(1+i t \lambda^{-1}\right)-i \ln \Gamma\left(1+\lambda^{-1}\right) \\
G_{\varepsilon}^{(r)}(t) & =\left(\frac{i}{\lambda}\right)^{r} \psi^{(r-1)}\left(1+i t \lambda^{-1}\right), r>1 .
\end{aligned}
$$

E para o caso da distribuição log-gama

$$
G_{\varepsilon}(t)=\ln \Gamma(\lambda+i t)-i t \ln \lambda-\ln \Gamma(\lambda)
$$

com derivadas dadas por

$$
\begin{aligned}
G_{\varepsilon}^{\prime}(t) & =i \psi(\lambda+i t)-i \ln \lambda \\
G_{\varepsilon}^{(r)}(t) & =i^{r} \psi^{(r-1)}(\lambda+i t), r>1 .
\end{aligned}
$$

Nas expressões acima temos que

$$
\psi(z)=\frac{\Gamma^{\prime}(z)}{\Gamma(z)}
$$

representa a função digama com argumento complexo, e

$$
\psi^{(r)}(z)=\frac{d^{r}}{d z^{r}} \psi(z)
$$

representa a função poligama com argumento complexo ${ }^{10}$.

Prova. A demonstração desta proposição encontra-se no apêndice $C$.

Podemos agora obter os cumulantes através da seguintes proposição.

\footnotetext{
${ }^{10}$ Para mais detalhes destas funções consulte, por exemplo, Abramowitz e Stegun (1972).
} 
Proposição 4.10 (Cumulantes de $\varepsilon_{j}$ ) Seja $\varepsilon_{j}$ uma variável aleatória com as funções densidade de probabilidade estabelecidas na prop. 4.7. Neste caso, os cumulantes da distribuição de $\varepsilon_{j}$ são dados para o caso log-exponencial por

$$
\begin{gathered}
\kappa_{1}=\psi(1)=-\gamma, \\
\kappa_{r}=\psi^{(r)}(1), r>1 .
\end{gathered}
$$

Para o caso da distribuição log-Weibull temos

$$
\begin{gathered}
\kappa_{1}=\frac{\psi(1)}{\lambda}-\ln \Gamma\left(1+\lambda^{-1}\right), \\
\kappa_{r}=\frac{\psi^{(r)}(1)}{\lambda^{r+1}}, r>1 .
\end{gathered}
$$

E para o caso da distribuição log-gama

$$
\begin{gathered}
\kappa_{1}=\psi(\lambda)-\ln \lambda, \\
\kappa_{r}=\psi^{(r)}(\lambda), r>1 .
\end{gathered}
$$

Nas expressões acima temos que $\gamma \approx 0.57721566490153286060 \cdots$ é a constante de Euler, e $\psi^{(1)}(1)=\pi^{2} / 6$.

Prova. Para obter o r-ésimo cumulante basta utilizar o resultado (4.49), isto é, o r-ésimo cumulante é dado pelo r-ésima derivada da função geratriz de cumulantes calculada em $t=0$ dividido por $i^{r}$.

Os momentos não centrais $\mu_{r}^{\prime}=E\left[\varepsilon^{r}\right]$ podem ser obtidos a partir dos cumulantes através da seguinte relação ${ }^{11}$ :

$$
\mu_{r}^{\prime}=\sum_{m=1}^{r} \sum_{\pi}\left(\frac{\kappa_{p_{1}}}{p_{1} !}\right)^{\pi_{1}}\left(\frac{\kappa_{p_{2}}}{p_{2} !}\right)^{\pi_{2}} \cdots\left(\frac{\kappa_{p_{m}}}{p_{m} !}\right)^{\pi_{m}} \frac{r !}{\pi_{1} ! \pi_{2} ! \cdots \pi_{m} !},
$$

onde o segundo somatório se extende para todos os valores não negativos dos $\pi^{\prime} s$ tais que

$$
p_{1} \pi_{1}+p_{2} \pi_{2}+\cdots+p_{m} \pi_{m}=r
$$

Aqui, vamos apresentar somente as relações para os quatro primeiros momentos não centrais,

\footnotetext{
${ }^{11}$ Para mais detalhes veja Stuart e Ord (1994), §3.14, pg. 87.
} 
como obtidos por Stuart e Ord (1994):

$$
\begin{aligned}
& \mu_{1}^{\prime}=\kappa_{1} \\
& \mu_{2}^{\prime}=\kappa_{2}+\kappa_{1}^{2} \\
& \mu_{3}^{\prime}=\kappa_{3}+3 \kappa_{2} \kappa_{1}+\kappa_{1}^{3} \\
& \mu_{4}^{\prime}=\kappa_{4}+4 \kappa_{3} \kappa_{1}+3 \kappa_{2}^{2}+6 \kappa_{2} \kappa_{1}^{2}+\kappa_{1}^{4}
\end{aligned}
$$

Entretanto é mais conveniente obter os momentos centrais $\mu_{r}=E\left[\left(\varepsilon-\mu_{\varepsilon}\right)^{r}\right], \mu_{\varepsilon}=\mu_{1}^{\prime}=$ $\kappa_{1}$, em função dos cumulantes, como obtidos por Stuart e Ord (1994):

$$
\begin{aligned}
& \mu_{2}=\kappa_{2}, \\
& \mu_{3}=\kappa_{3}, \\
& \mu_{4}=\kappa_{4}+10 \kappa_{2}^{2} .
\end{aligned}
$$

Proposição 4.11 (4 primeiros momentos de $\varepsilon_{j}$ ) Seja $\varepsilon_{j}$ uma variável aleatória com as funções densidade de probabilidade estabelecidas na prop. 4.7. Neste caso, os quatro primeiros momentos centrais da distribuição de $\varepsilon_{j}$ são dados para o caso log-exponencial por

$$
\begin{aligned}
& \mu_{1}=\psi(1)=-\gamma \\
& \mu_{2}=\psi^{(1)}(1)=\frac{\pi^{2}}{6} \\
& \mu_{3}=\psi^{(2)}(1) \\
& \mu_{4}=\psi^{(3)}(1)+\frac{5 \pi^{4}}{18}
\end{aligned}
$$

Para o caso da distribuição log-Weibull temos

$$
\begin{aligned}
& \mu_{1}=\frac{\psi(1)}{\lambda}-\ln \Gamma\left(1+\lambda^{-1}\right)=-\frac{\gamma}{\lambda}-\ln \Gamma\left(1+\lambda^{-1}\right) \\
& \mu_{2}=\frac{\psi^{(1)}(1)}{\lambda^{2}}=\frac{\pi^{2}}{6 \lambda^{2}} \\
& \mu_{3}=\frac{\psi^{(2)}(1)}{\lambda^{3}} \\
& \mu_{4}=\frac{\psi^{(3)}(1)}{\lambda^{4}}+\frac{5 \pi^{4}}{18 \lambda^{4}} .
\end{aligned}
$$


E para o caso da distribuição log-gama

$$
\begin{aligned}
& \mu_{1}=\psi(\lambda)-\ln \lambda, \\
& \mu_{2}=\psi^{(1)}(\lambda), \\
& \mu_{3}=\psi^{(2)}(\lambda), \\
& \mu_{4}=\psi^{(3)}(\lambda)+10\left[\psi^{(1)}(\lambda)\right]^{2} .
\end{aligned}
$$

Prova. Para obter as relações acima basta aplicar os resultados da prop. 4.10 aos resultados 4.71) $a$ (4.73).

O coeficiente de assimetria $\gamma_{1}$ e o coeficiente de excesso de curtose $\gamma_{2}$ são dados por

$$
\begin{aligned}
\gamma_{1} & =\frac{\mu_{3}}{\mu_{2}^{3 / 2}}, \\
\gamma_{2} & =\frac{\mu_{4}}{\mu_{2}^{2}}-3 .
\end{aligned}
$$

Note que, para o caso da distribuição log-Weibull, tanto o coeficiente de assimetria, quanto o coeficiente de excesso de curtose, independem do parâmetro $\lambda$, e coincidem com os respectivos coeficientes da distribuição log-exponencial:

$$
\begin{aligned}
& \gamma_{1}=\frac{\frac{\psi^{(2)}(1)}{\lambda^{3}}}{\left(\frac{\pi^{2}}{6 \lambda^{2}}\right)^{3 / 2}}=\frac{\psi^{(2)}(1)}{\left(\pi^{2} / 6\right)^{3}}, \\
& \gamma_{2}=\frac{\frac{\psi^{(3)}(1)}{\lambda^{4}}+\frac{5 \pi^{4}}{18 \lambda^{4}}}{\left(\frac{\pi^{2}}{6 \lambda^{2}}\right)^{2}}-3=\frac{\psi^{(3)}(1)}{\left(\pi^{2} / 6\right)^{2}}+7 .
\end{aligned}
$$

As Figuras (4.3) e (4.4) ilustram os coeficientes de assimetria e excesso de curtose em função de $\lambda$ para as distribuições log-exponencial, log-Weibull e log-gama.

\subsection{Função característica conjunta ("função poli-característica")}

Para estimar o modelo SCD utilizando o método da ECF precisamos calcular a "função poli-característica" (PCF) associada com a distribuição conjunta de um bloco sequencial de tamanho $p+1$ das log-durações. Mas antes de obter este resultado, vamos calcular os $\psi_{j}$ para blocos de tamanho $p+1$, através da seguinte proposição: 


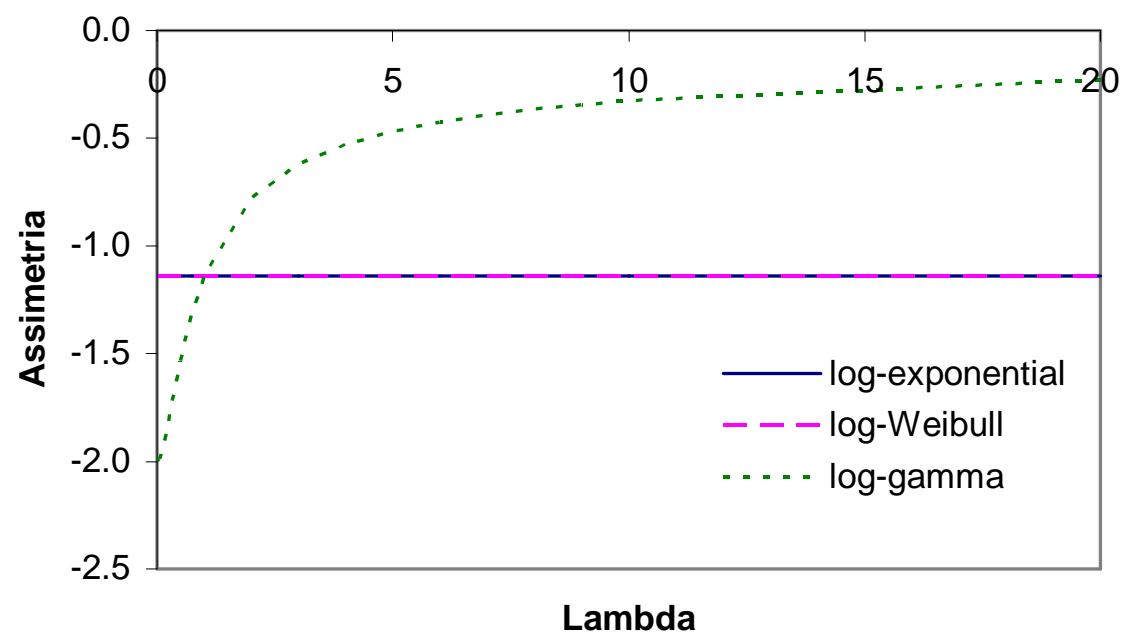

Figura 4.3: Coeficiente de assimetria $\gamma_{1}$ em função de $\lambda$, para as distribuições log-exponencial, log-Weibull e log-gama.

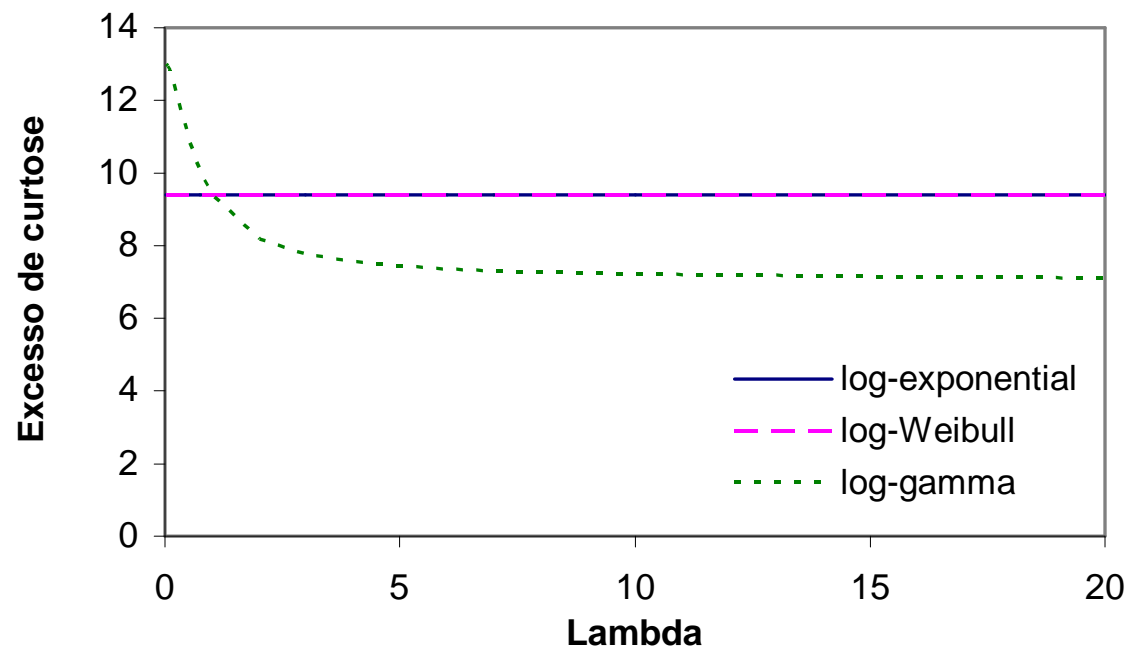

Figura 4.4: Coeficiente de excesso de curtose $\gamma_{2}$ em função de $\lambda$, para as distribuições logexponencial, log-Weibull e log-gama.

Proposição 4.12 (Variável latente $\psi_{j+k}, k$ passos adiante de $\psi_{j}$ ) A variável latente $\psi_{j+k}, k$ passos adiante de $\psi_{j}$ definida em (4.5) do modelo (4.4)-4.8), é dada por:

$$
\psi_{j+k}=\frac{1-\phi^{k}}{1-\phi} \delta+\phi^{k} \psi_{j}+\sigma_{\eta} \sum_{l=0}^{k-1} \phi^{l} \eta_{j+k-l}, \quad j=1, \cdots, T-k .
$$

Prova. A demonstração desta proposição encontra-se no apêndice $\square$. 
Seguindo o procedimento de ECF conjunta sugerido por Knight e Yu (2002), vamos chamar de $Z_{j}$ o vetor de blocos sobrepostos de log-durações $\left(Y_{j}, Y_{j+1}, \cdots, Y_{j+p}\right)^{\prime}, j=1, \cdots, T-p$. A função característica conjunta do bloco é dada por:

$$
\varphi_{z}(r)=E\left[\exp \left(i r^{\prime} Z_{j}\right)\right]
$$

em que $r$ é um vetor real $(p+1) \times 1$. Para o modelo SCD temos a seguinte proposição

Proposição 4.13 (Função poli-característica do modelo SCD) Seja o modelo SCD definido pelas equações (4.4)-4.5), e seja $Z_{j}$ o vetor de blocos sobrepostos de log-durações $\left(Y_{j}, Y_{j+1}, \cdots, Y_{j+p}\right)^{\prime}$. Neste caso, a função poli-característica do vetor $Z_{j}$ é dada por

$$
\begin{aligned}
\varphi_{z}(r)= & \exp \left\{\frac{i \delta}{1-\phi} \sum_{\zeta=1}^{p+1} r_{\zeta}-\frac{\sigma_{\eta}^{2}}{2\left(1-\phi^{2}\right)}\left(\sum_{\zeta=1}^{p+1} \phi^{\zeta-1} r_{\zeta}\right)^{2}-\right. \\
& \left.-\sum_{j=2}^{p+1} \frac{\sigma_{\eta}^{2}}{2}\left(\sum_{\zeta=j}^{p+1} \phi^{\zeta-j} r_{\zeta}\right)^{2}\right\} \prod_{j=1}^{p+1} \varphi_{\varepsilon}\left(r_{j}\right),
\end{aligned}
$$

onde $r=\left(r_{1}, \cdots, r_{p+1}\right)^{\prime} \in \mathbb{R}^{p+1}$ e $\varphi_{\varepsilon}(s)$ é a função característica de $\varepsilon_{j}=\ln \xi_{j}$, fornecida pela proposição 4.8.

Prova. A demonstração desta proposição encontra-se no apêndice $\square$.

\subsection{Verossimilhança amostral para o modelo SCD}

A maneira mais natural de estimar os parâmetros do modelo SCD seria através da maximização da função de verossimilhança. Para obtê-la, vamos assumir que possuímos uma amostra de tamanho $T$ das durações. Vamos chamar de $X=\left(X_{1}, \cdots, X_{T}\right)^{\prime}$ e de $\Psi=\left(\psi_{1}, \cdots, \psi_{T}\right)^{\prime}$. Pela hipótese de independência condicional temos que a função densidade de probabilidade condicional de $X$ dado $\Psi$ é

$$
p(x \mid \psi, \theta)=\prod_{j=1}^{T} p\left(x_{j} \mid \psi_{j}\right) .
$$

A função densidade de probabilidade conjunta de $X$ e $\Psi$ pode ser obtida através do condicionamento de $\psi_{j}$ em $\psi_{j-1}$

$$
p(x, \psi \mid \theta)=\prod_{j=1}^{T} p\left(x_{j} \mid \psi_{j}\right) p\left(\psi_{j} \mid \psi_{j-1}, \theta\right) p\left(\psi_{1} \mid \theta\right) .
$$

Finalmente, a função de densidade da amostra pode ser obtida através da eliminação do vetor de variáveis latentes $\Psi$ através da seguinte integração $T$-dimensional

$$
p(x \mid \theta)=\int_{-\infty}^{\infty} \cdots \int_{-\infty}^{\infty} p(x, \psi \mid \theta) d \psi_{1} \cdots d \psi_{T}
$$


Bauwens e Veredas (2004) colocam que "a principal dificuldade com o modelo SCD é a sua estimação, porque ao contrário do modelo $\mathrm{ACD}$, não é fácil calcular sua função de verossimilhança: a variável latente tem que ser integrada." A equação (4.90) indica que a obtenção da verossimilhança amostral requer uma integração $T$-dimensional. A questão fundamental é que, com as distribuições propostas, a integral em (4.90) não pode ser calculada analiticamente, e, portanto, métodos numéricos de integração devem ser utilizados. Acontece que, como $T$ é potencialmente muito grande (da ordem de milhares, ou maior), os métodos numéricos de quadratura são muito lentos e acumulam erros, dado que as funções integrandas são estritamente positivas. Para lidar com este problema, métodos mais sofisticados de integração multidimensional devem ser empregrados. Em particular, Strickland et al. (2006) empregam um método de MCMC (Markov Chain Monte Carlo) para estimar o modelo SCD.

Para estimação do modelo SCD Bauwens e Veredas (2004) empregam o método de quasemáxima verossimilhança (QML - quasi maximum likelihood), que consiste basicamente em utilizar o método da máxima verossimilhança como se as distribuições fossem Gaussianas, de maneira a poder utilizar o filtro de Kalman para estimação da variável latente. Note que o uso das equações (4.32) e (4.33) permite escrever o modelo no formato de espaço de estados:

$$
\begin{gathered}
\psi_{j}=\delta+\phi \psi_{j-1}+\sigma_{\eta} \eta_{t} \\
y_{j}=\psi_{j}+\varepsilon_{j},
\end{gathered}
$$

em que (4.91) é a equação de estado ou transição, e (4.92) é a equação de observação ou de mensuração. A vantagem da colocação do modelo neste formato é permitir o uso do algoritmos especializados (como o filtro de Kalman) para estimação das variáveis latentes. Esta abordagem será tratada no Capítulo 6, em que aplicamos o algoritmo do filtro de Kalman para o modelo de interesse, utilizando o algoritmo QML como estimador.

\subsection{Sumário}

Neste capítulo revimos o modelo ACD de Engle e Russell (1998) e o modelo SCD de Bauwens e Veredas (2004). Especificamos o particular modelo SCD de interesse e para suas distribuições dos log-erros obtivemos uma série de resultados:

- função característica;

- função geratriz de cumulantes;

- cumulantes;

- quatro primeiros momentos centrais.

Obtivemos, também, a função "poli-característica" para o modelo SCD de interesse e relatamos a dificuldade do uso do método da verossimilhança para estimação dos parâmetros, devido à necessidade de uma integração $T$-dimensional. 
Finalmente, notamos que o uso das log-durações fornece um modelo no formato de espaço de estados linear, o que permitiria o uso de algoritmos como o filtro de Kalman para estimação das variáveis latentes.

O foco deste trabalho, no entanto, é a estimação do modelo SCD através do método da função característica empírica (ECF), o que será feito no próximo capítulo. 



\section{Capítulo 5}

\section{Estimação do modelo SCD utilizando a ECF}

No capítulo anterior obtivemos a função poli-característica do modelo de duração condicional estocástica. Neste capítulo vamos obter algumas propriedades dos estimadores calculados através dos métodos CECF e DECF para estimação do modelo SCD.

\subsection{Estimação dos parâmetros através da CECF}

Para estimar os parâmetros usando o método CECF proposto por Knight e Yu (2002), temos que resolver o seguinte problema de otimização:

$$
\widehat{\theta}_{n}=\arg \min _{\theta \in \Theta} \int_{\mathbb{R}^{p+1}}\left|\varphi_{n}(r)-\varphi_{z}(r, \theta)\right|^{2} g(r) d r .
$$

O estimador $\widehat{\theta}_{n}$ é um estimador extremo ${ }^{1}$, pois é obtido através da minimização de uma função $Q_{n}(\theta)$ da distância ponderada entre a função característica empírica e a função característica:

$$
Q_{n}(\theta)=\int_{\mathbb{R}^{p+1}}\left|\varphi_{n}(r)-\varphi_{z}(r, \theta)\right|^{2} g(r) d r .
$$

Utilizando (2.8) podemos reescrever (5.2) acima como:

\footnotetext{
${ }^{1}$ Veja apêndice $\mathrm{A}$
} 


$$
\begin{aligned}
Q_{n}(\theta) & =\int_{\mathbb{R}^{p+1}}\left|\varphi_{n}(r)-\varphi_{z}(r, \theta)\right|^{2} g(r) d r \\
& =\int_{\mathbb{R}^{p+1}}\left|\frac{1}{n} \sum_{j=1}^{n} e^{i r^{\prime} z_{j}}-\varphi_{z}(r, \theta)\right|^{2} g(r) d r \\
& =\int_{\mathbb{R}^{p+1}}\left|\frac{1}{n} \sum_{j=1}^{n} e^{i r^{\prime} z_{j}}-\frac{1}{n} \sum_{j=1}^{n} \varphi_{z}(r, \theta)\right|^{2} g(r) d r \\
& =\int_{\mathbb{R}^{p+1}}\left|\frac{1}{n} \sum_{j=1}^{n}\left\{e^{i r^{\prime} z_{j}}-\varphi_{z}(r, \theta)\right\}\right|^{2} g(r) d r \\
& =\int_{\mathbb{R}^{p+1}}\left|\frac{1}{n} \sum_{j=1}^{n}\left\{\cos \left(r^{\prime} z_{j}\right)-\operatorname{Re} \varphi_{z}(r, \theta)+i\left(\sin \left(r^{\prime} z_{j}\right)-\operatorname{Im} \varphi_{z}(r, \theta)\right)\right\}\right|^{2} g(r) d r .
\end{aligned}
$$

Em particular vamos utilizar $g(r)=\exp \left(-r^{\prime} r\right)$, de maneira a podermos efetuar a integração multidimensional acima através do método de quadratura de Gauss-Hermite (ver Press et al. (1997)), mas limitando $r$ à uma esfera multidimensional como sugerido por Jäckel (2005), de maneira a aumentar a eficiência computacional.

Knight e Yu (2002) estabelecem algumas condições de regularidade, descritas no Capítulo 3. sob as quais o estimador acima é consistente e assintoticamente normalmente distribuído para o caso dependente com o seguinte resultado:

$$
\sqrt{n}\left(\widehat{\theta}_{n}-\theta\right) \underset{n \rightarrow \infty}{\stackrel{d}{\longrightarrow}} N\left(0, B(\theta)^{-1} A(\theta) B(\theta)^{-1}\right)
$$

em que a matriz $B(\theta)$ é dada pela integral $p+1$-dimensional:

$$
B(\theta)=\int_{\mathbb{R}^{p+1}}\left(\frac{\partial \operatorname{Re} \varphi_{z}(r, \theta)}{\partial \theta} \frac{\partial \operatorname{Re} \varphi_{z}(r, \theta)}{\partial \theta^{\prime}}+\frac{\partial \operatorname{Im} \varphi_{z}(r, \theta)}{\partial \theta} \frac{\partial \operatorname{Im} \varphi_{z}(r, \theta)}{\partial \theta^{\prime}}\right) g(r) d r .
$$

Knight et al. (2002) fornecem a seguinte expressão para $A(\theta)$ :

$$
A(\theta)=\lim _{n \rightarrow \infty} \frac{1}{n} E\left\{\sum_{j=1}^{n} \sum_{k=1}^{n} K\left(z_{j}, \theta\right) K\left(z_{k}, \theta\right)^{\prime}\right\},
$$

em que o vetor aleatório $K\left(z_{j}, \theta\right)$ é uma variável auxiliar proveniente da derivação de $Q_{n}(\theta)$ em relação a $\theta$ :

$$
\frac{\partial Q_{n}(\theta)}{\partial \theta}=-\frac{2}{n} \sum_{j=1}^{n} K\left(z_{j}, \theta\right)
$$


sendo:

$$
\begin{aligned}
K\left(z_{j}, \theta\right)= & \int_{\mathbb{R}^{p+1}}\left\{\left(\cos \left(r^{\prime} z_{j}\right)-\operatorname{Re} \varphi_{z}(r, \theta)\right) \frac{\partial \operatorname{Re} \varphi_{z}(r, \theta)}{\partial \theta}+\right. \\
& \left.+\left(\sin \left(r^{\prime} z_{j}\right)-\operatorname{Im} \varphi_{z}(r, \theta)\right) \frac{\partial \operatorname{Im} \varphi_{z}(r, \theta)}{\partial \theta}\right\} g(r) d r .
\end{aligned}
$$

A derivação de (5.4) a (5.8) é feita no apêndiceD. Infelizmente a equação (5.6) não fornece diretamente um estimador para a matriz $A(\theta)$. Knight e Yu (2002) não explicitam o estimador utilizado para $A(\theta)$, mas sugerem a obtenção de um estimador consistente utilizando os métodos de Andrews (1991) e Newey e West (1994), isto é, um estimador consistente e robusto à heteroscedasticidade e à autocorrelação para a matriz de covariâncias de $K\left(z_{j}, \theta\right)$, possivelmente através do estimador $\widehat{K}\left(z_{j}, \theta\right)=K\left(z_{j}, \widehat{\theta}_{n}\right)$ :

$$
\begin{aligned}
\widehat{K}\left(z_{j}, \theta\right)= & K\left(z_{j}, \widehat{\theta}_{n}\right) \\
= & \int_{\mathbb{R}^{p+1}}\left\{\left(\cos \left(r^{\prime} z_{j}\right)-\operatorname{Re} \varphi_{z}\left(r, \widehat{\theta}_{n}\right)\right) \frac{\partial \operatorname{Re} \varphi_{z}\left(r, \widehat{\theta}_{n}\right)}{\partial \theta}+\right. \\
& \left.+\left(\sin \left(r^{\prime} z_{j}\right)-\operatorname{Im} \varphi_{z}\left(r, \widehat{\theta}_{n}\right)\right) \frac{\partial \operatorname{Im} \varphi_{z}\left(r, \widehat{\theta}_{n}\right)}{\partial \theta}\right\} g(r) d r .
\end{aligned}
$$

Um outro possível estimador para a matriz $A\left(\theta_{0}\right)$ é dado na proposição a seguir.

Proposição 5.1 (estimador para $A\left(\theta_{0}\right)$ ) Um estimador para a matriz $A\left(\theta_{0}\right)$ é dado por

$$
\begin{aligned}
\widehat{A}(\theta)= & \int_{\mathbb{R}^{p+1}} \int_{\mathbb{R}^{p+1}}\left(\frac{\partial \operatorname{Re} \varphi_{z}\left(r, \widehat{\theta}_{n}\right)}{\partial \theta} \frac{\partial \operatorname{Re} \varphi_{z}\left(s, \widehat{\theta}_{n}\right)}{\partial \theta^{\prime}} \Omega_{R R, m}(r, s)+\right. \\
& +\frac{\partial \operatorname{Re} \varphi_{z}\left(r, \widehat{\theta}_{n}\right)}{\partial \theta} \frac{\partial \operatorname{Im} \varphi_{z}\left(s, \widehat{\theta}_{n}\right)}{\partial \theta^{\prime}} \Omega_{R I, m}(r, s)+ \\
& +\frac{\partial \operatorname{Im} \varphi_{z}\left(r, \widehat{\theta}_{n}\right)}{\partial \theta} \frac{\partial \operatorname{Re} \varphi_{z}\left(s, \widehat{\theta}_{n}\right)}{\partial \theta^{\prime}} \Omega_{I R, m}(r, s) \\
& \left.+\frac{\partial \operatorname{Im} \varphi_{z}\left(r, \widehat{\theta}_{n}\right)}{\partial \theta} \frac{\partial \operatorname{Im} \varphi_{z}\left(s, \widehat{\theta}_{n}\right)}{\partial \theta^{\prime}} \Omega_{I I, m}(r, s)\right) g(r) g(s) d r d s,
\end{aligned}
$$

em que a matriz $\Omega(r, s)$ é formada pela seguinte partição:

$$
\Omega(r, s)=\left[\begin{array}{cc}
\Omega_{R R, m}(r, s) & \Omega_{R I, m}(r, s) \\
\Omega_{I R, m}(r, s) & \Omega_{I I, m}(r, s)
\end{array}\right],
$$


$e$

$$
\begin{aligned}
\Omega_{R R, m}(r, s)= & \frac{1}{2}\{\operatorname{Re} \varphi(r-s)+\operatorname{Re} \varphi(r+s)\}-m \operatorname{Re} \varphi(r) \operatorname{Re} \varphi(s)+ \\
& +\frac{1}{2 m} \sum_{k=1}^{m-1}(m-k)\left\{\operatorname{Re} \Lambda_{k}(r,-s)+\operatorname{Re} \Lambda_{k}(r, s)+\operatorname{Re} \Lambda_{k}(-s, r)+\operatorname{Re} \Lambda_{k}(s, r)\right\},
\end{aligned}
$$

$$
\begin{aligned}
\Omega_{R I, m}(r, s)= & \frac{1}{2}\{-\operatorname{Im} \varphi(r-s)+\operatorname{Im} \varphi(r+s)\}-m \operatorname{Re} \varphi(r) \operatorname{Im} \varphi(s)+ \\
& +\frac{1}{2 m} \sum_{k=1}^{m-1}(m-k)\left\{-\operatorname{Im} \Lambda_{k}(r,-s)+\operatorname{Im} \Lambda_{k}(r, s)-\operatorname{Im} \Lambda_{k}(-s, r)+\operatorname{Im} \Lambda_{k}(s, r)\right\},
\end{aligned}
$$

$$
\begin{aligned}
\Omega_{I R, m}(r, s)= & \frac{1}{2}\{\operatorname{Im} \varphi(r-s)+\operatorname{Im} \varphi(r+s)\}-m \operatorname{Im} \varphi(r) \operatorname{Re} \varphi(s)+ \\
& +\frac{1}{2 m} \sum_{k=1}^{m-1}(n-k)\left\{\operatorname{Im} \Lambda_{k}(r,-s)+\operatorname{Im} \Lambda_{k}(r, s)+\operatorname{Im} \Lambda_{k}(-s, r)+\operatorname{Im} \Lambda_{k}(s, r)\right\},
\end{aligned}
$$

$$
\begin{aligned}
\Omega_{I I, m}(r, s)= & \frac{1}{2}\{\operatorname{Re} \varphi(r-s)-\operatorname{Re} \varphi(r+s)\}-m \operatorname{Im} \varphi(r) \operatorname{Im} \varphi(s)+ \\
& +\frac{1}{2 m} \sum_{k=1}^{m-1}(m-k)\left\{\operatorname{Re} \Lambda_{k}(r,-s)-\operatorname{Re} \Lambda_{k}(r, s)+\operatorname{Re} \Lambda_{k}(-s, r)-\operatorname{Re} \Lambda_{k}(s, r)\right\} .
\end{aligned}
$$

em que

$$
\Lambda_{k}(r, s)=E\left[e^{i r^{\prime} Z_{j}+i s^{\prime} Z_{j+k}}\right]
$$

e $m \leq n$ é um número suficientemente grande para que as séries acima tenham convergido aproximadamente.

Prova. A obtenção deste estimador encontra-se no apêndice $\square$, onde é chamado de $A_{n}^{(3)}\left(\theta_{0}\right)$.

Finalmente, se chamarmos de $V=B(\theta)^{-1} A(\theta) B(\theta)^{-1}$, podemos obter um estimador consistente para $V$ através de:

$$
\widehat{V}=\widehat{B}(\theta)^{-1} \widehat{A}(\theta) \widehat{B}(\theta)^{-1},
$$

em que $\widehat{B}(\theta)=B\left(\widehat{\theta}_{n}\right)$, isto é, a matriz $B$ calculada no estimador CECF de $\theta$ :

$B\left(\widehat{\theta}_{n}\right)=\int_{\mathbb{R}^{p+1}}\left(\frac{\partial \operatorname{Re} \varphi_{z}\left(r, \widehat{\theta}_{n}\right)}{\partial \theta} \frac{\partial \operatorname{Re} \varphi_{z}\left(r, \widehat{\theta}_{n}\right)}{\partial \theta^{\prime}}+\frac{\partial \operatorname{Im} \varphi_{z}\left(r, \widehat{\theta}_{n}\right)}{\partial \theta} \frac{\partial \operatorname{Im} \varphi_{z}\left(r, \widehat{\theta}_{n}\right)}{\partial \theta^{\prime}}\right) g(r) d r$

e $\widehat{A}(\theta)$ pode ser estimada por (5.10). 
Para o caso específico do modelo de duração condicional estocástica podemos calcular as funções $\Lambda_{k}(r, s)$ através da seguinte proposição:

Proposição 5.2 (Função $\Lambda_{k}(r, s)$ para o modelo SCD) Seja o modelo $S C D$ definido por 4.4) a 4.8). Chamemos $w(r, s)$ o vetor $(p+k+1) \times 1$ dado por

$$
w(r, s)=\left\{\begin{array}{cl}
\left(r_{1}, \cdots, r_{k}, r_{k+1}+s_{1}, \cdots, r_{p+1}+s_{p-k+1}, s_{p-k+2}, \cdots, s_{p+1}\right)^{\prime} & \text { se } k \leq p \\
\left(r_{1}, \cdots, r_{p+1}, 0, \cdots, 0, s_{1}, \cdots, s_{p+1}\right)^{\prime} & \text { se } k>p
\end{array}\right.
$$

em que $r, s \in \mathbb{R}^{k+1}$. Utilizando a função poli-característica (4.87) podemos escrever (5.16) como:

$$
\begin{aligned}
\Lambda_{k}(r, s)= & \exp \left\{\frac{i \delta}{1-\phi} \sum_{\zeta=1}^{p+k+1} w_{\zeta}-\frac{\sigma_{\eta}^{2}}{2\left(1-\phi^{2}\right)}\left(\sum_{\zeta=1}^{p+k+1} \phi^{\zeta-1} w_{\zeta}\right)^{2}-\right. \\
& \left.-\sum_{j=2}^{p+k+1} \frac{\sigma_{\eta}^{2}}{2}\left(\sum_{\zeta=j}^{p+k+1} \phi^{\zeta-j} w_{\zeta}\right)^{2}\right\} \prod_{\zeta=1}^{p+k+1} \varphi_{\varepsilon}\left(w_{\zeta}\right),
\end{aligned}
$$

em que $w_{\zeta}$ designa o $\zeta$-ésimo elemento do vetor $w(r, s)$.

Prova. A demonstração desta proposição encontra-se no apêndice $\square$.

Vamos agora verificar qual o comportamento assintótico de $\Omega_{n}(r, s)$ para o modelo SCD. Para isso vamos inicialmente verificar qual o comportamento da variável $\psi_{j+k}$. Utilizando (4.86) podemos escrever:

$$
\psi_{j+k}=\frac{1-\phi^{k}}{1-\phi} \delta+\phi^{k} \psi_{j}+\sigma_{\eta} \sum_{l=0}^{k-1} \phi^{l} \eta_{j+k-l}
$$

A função característica de $\psi_{j+k}$ pode então ser calculada por:

$$
\begin{aligned}
\varphi_{\psi_{j+k}}(t) & =E\left[\exp \left(i t \psi_{j+k}\right)\right]= \\
& =E\left[\exp \left\{i t \frac{1-\phi^{k}}{1-\phi} \delta+i t \phi^{k} \psi_{j}+i t \sigma_{\eta} \sum_{l=0}^{k-1} \phi^{l} \eta_{j+k-l}\right\}\right]= \\
& =\exp \left(i t \delta \frac{1-\phi^{k}}{1-\phi}\right) \cdot E\left[\exp \left(i t \phi^{k} \psi_{j}\right)\right] \cdot \prod_{l=0}^{k-1} E\left[\exp \left(i t \sigma_{\eta} \phi^{l} \eta_{j+k-l}\right)\right]= \\
& =\exp \left(i t \delta \frac{1-\phi^{k}}{1-\phi}\right) \cdot \varphi_{\psi_{j}}\left(t \phi^{k}\right) \cdot \prod_{l=0}^{k-1} \varphi_{\eta}\left(t \sigma_{\eta} \phi^{l} \eta_{j+k-l}\right)= \\
& =\exp \left(i t \delta \frac{1-\phi^{k}}{1-\phi}\right) \cdot \exp \left(\frac{i t \delta \phi^{k}}{1-\phi}-\frac{\sigma_{\eta}^{2} t^{2} \phi^{2 k}}{2\left(1-\phi^{2}\right)}\right) \cdot \sum_{l=0}^{k-1} \exp \left(-\frac{t^{2} \sigma_{\eta}^{2} \phi^{2 l}}{2}\right)= \\
& =\exp \left(i t \delta \frac{1-\phi^{k}}{1-\phi}+\frac{i t \delta \phi^{k}}{1-\phi}-\frac{\sigma_{\eta}^{2} t^{2} \phi^{2 k}}{2\left(1-\phi^{2}\right)}-\frac{t^{2} \sigma_{\eta}^{2}}{2} \sum_{l=0}^{k-1} \phi^{2 l}\right)
\end{aligned}
$$


Podemos observar por (5.20) que o limite de $\varphi_{\psi_{j+k}}(t)$ quando $k \rightarrow \infty$, isto é, quando o intervalo entre $j$ e $j+k$ fica muito grande, é dado por

$$
\lim _{k \rightarrow \infty} \varphi_{\psi_{j+k}}(t)=\exp \left(\frac{i t \delta}{1-\phi}-\frac{t^{2} \sigma_{\eta}^{2}}{2\left(1-\phi^{2}\right)}\right)=\varphi_{\psi}(t) .
$$

Vamos agora obter a função característica de $\psi_{j+k}$ dado $\psi_{j}$. Neste caso temos

$$
\begin{aligned}
\varphi_{\psi_{j+k} \mid \psi_{j}}(t) & =E\left[\exp \left(i t \psi_{j+k}\right) \mid \psi_{j}\right]= \\
& =E\left[\exp \left\{i t \frac{1-\phi^{k}}{1-\phi} \delta+i t \phi^{k} \psi_{j}+i t \sigma_{\eta} \sum_{l=0}^{k-1} \phi^{l} \eta_{j+k-l}\right\}\right]= \\
& =\exp \left(i t \delta \frac{1-\phi^{k}}{1-\phi}\right) \cdot \exp \left(i t \phi^{k} \psi_{j}\right) \cdot \prod_{l=0}^{k-1} E\left[\exp \left(i t \sigma_{\eta} \phi^{l} \eta_{j+k-l}\right)\right]= \\
& =\exp \left(i t \phi^{k} \psi_{j}\right) \cdot \exp \left(i t \delta \frac{1-\phi^{k}}{1-\phi}\right) \cdot \prod_{l=0}^{k-1} \varphi_{\eta}\left(t \sigma_{\eta} \phi^{l} \eta_{j+k-l}\right)= \\
& =\exp \left(i t \delta \frac{1-\phi^{k}}{1-\phi}\right) \cdot \sum_{l=0}^{k-1} \exp \left(-\frac{t^{2} \sigma_{\eta}^{2} \phi^{2 l}}{2}\right)= \\
& =\exp \left(i t \phi^{k} \psi_{j}+i t \delta \frac{1-\phi^{k}}{1-\phi}-\frac{t^{2} \sigma_{\eta}^{2}}{2} \sum_{l=0}^{k-1} \phi^{2 l}\right) .
\end{aligned}
$$

Note que,

$$
\lim _{k \rightarrow \infty} \varphi_{\psi_{j+k} \mid \psi_{j}}(t)=\exp \left(\frac{i t \delta}{1-\phi}-\frac{t^{2} \sigma_{\eta}^{2}}{2\left(1-\phi^{2}\right)}\right)=\varphi_{\psi}(t) .
$$

Logo, como a função característica de $\psi_{j+k}$ dado $\psi_{j}$, e, conseqüentemente, sua distribuição, não dependem de $\psi_{j}$ quando $k \rightarrow \infty$, concluimos que $\psi_{j+k}$ e $\psi_{j}$ são assintoticamente independentes. Neste caso, podemos definir $\Lambda_{\infty}(r, s)$ por:

$$
\begin{aligned}
\Lambda_{\infty}(r, s) & =\lim _{k \rightarrow \infty} \Lambda_{k}(r, s)= \\
& =\lim _{k \rightarrow \infty} E\left[\exp \left(i r^{\prime} z_{j}+i s^{\prime} z_{j+k}\right)\right]= \\
& =E\left[\exp \left(i r^{\prime} z_{j}\right)\right] \cdot E\left[\exp \left(i r^{\prime} z_{j+k}\right)\right]= \\
& =\varphi_{z}(r) \varphi_{z}(s) .
\end{aligned}
$$

A fim de visualizar o comportamento da matriz de covariâncias $\Omega_{n}(r, s)$ no modelo SCD graficamos na figura (5.1) as partições de $\Omega_{n}(r, s)$ conforme as equações (D.13) a (D.16). Para finalidade de comparação graficamos, também, o estimador para o caso i.i.d., $\Omega_{n}^{i i d}(r, s)$, dado pelas equações (3.17) a (3.20), que repetimos abaixo por conveniência: 

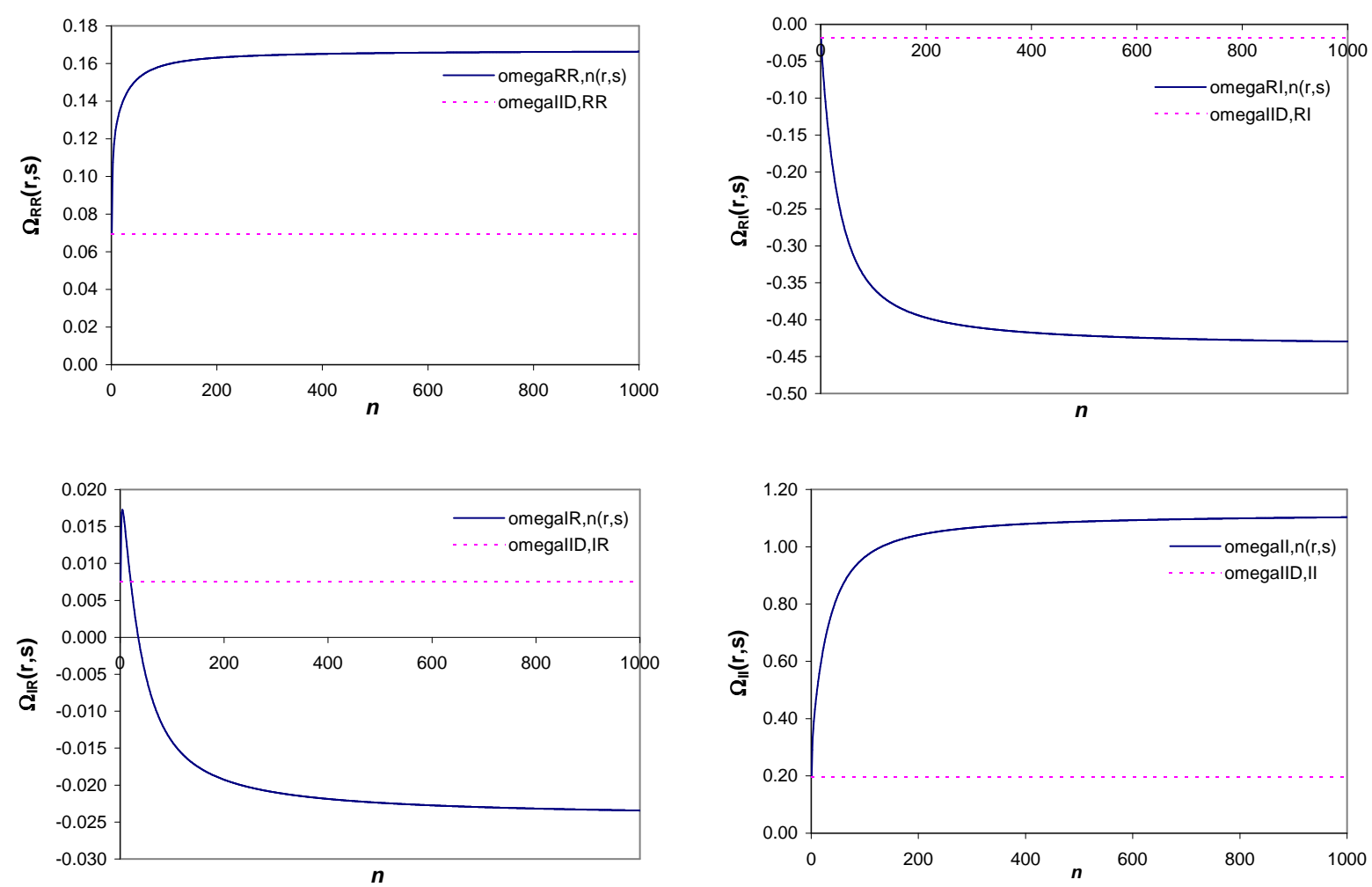

Figura 5.1: Matriz de covariância $\Omega_{n}(r, s)$ para o processo de duração estocástica condicional, em função do tamanho da amostra $(n)$. Os parâmetros do processo SCD são $\delta=0.033$, $\phi=0.95, \sigma_{\eta}=0.1$ e $\lambda=2$, com distribuição Weibull. Os pontos para cálculo da função característica (sorteados aleatoriamente) são $r=(0.891299,0.762097)^{\prime}$ e $s=(0.456468,0.018504)^{\prime}$. Para fim de comparação a figura também ilustra a matriz $\Omega_{n}^{i i d}(r, s)$ do processo IID .

$$
\begin{aligned}
& \Omega_{R R}^{i . i d .}(r, s)=\frac{1}{2}\left[\operatorname{Re} \varphi_{z}(r-s)+\operatorname{Re} \varphi_{z}(r+s)\right]-\operatorname{Re} \varphi_{z}(r) \operatorname{Re} \varphi_{z}(s), \\
& \Omega_{R I}^{i . i . d .}(r, s)=\frac{1}{2}\left[-\operatorname{Im} \varphi_{z}(r-s)+\operatorname{Im} \varphi_{z}(r+s)\right]-\operatorname{Re} \varphi_{z}(r) \operatorname{Im} \varphi_{z}(s), \\
& \Omega_{I R}^{i . i . d .}(r, s)=\frac{1}{2}\left[\operatorname{Im} \varphi_{z}(r-s)+\operatorname{Im} \varphi_{z}(r+s)\right]-\operatorname{Im} \varphi_{z}(r) \operatorname{Re} \varphi_{z}(s), \\
& \Omega_{I I}^{i . i . d .}(r, s)=\frac{1}{2}\left(\operatorname{Re} \varphi_{z}(r-s)-\operatorname{Re} \varphi_{z}(r+s)\right)-\operatorname{Im} \varphi_{z}(r) \operatorname{Im} \varphi_{z}(s) .
\end{aligned}
$$




\subsection{Estimação dos parâmetros através da DECF}

O estimador DECF $\widehat{\theta}_{n}$ é definido por

$$
\widehat{\theta}_{n}=\arg \min _{\theta \in \Theta} Q_{n}
$$

sendo a função $Q_{n}$ dada por

$$
Q_{n}=\left(V_{n}-V_{\theta}\right)^{\prime} W_{n}\left(V_{n}-V_{\theta}\right),
$$

em que $W_{n}$ é uma matriz $2 q \times 2 q$ simétrica, de posto $\operatorname{rank}\left(W_{n}\right) \leq 2 q$, e

$$
V_{n}-V_{\theta}=\left(\begin{array}{c}
u_{n}\left(r_{1}\right)-u\left(r_{1}\right) \\
\vdots \\
u_{n}\left(r_{q}\right)-u\left(r_{q}\right) \\
v_{n}\left(r_{1}\right)-v\left(r_{1}\right) \\
\vdots \\
v_{n}\left(r_{q}\right)-v\left(r_{q}\right)
\end{array}\right)_{2 q \times 1}
$$

em que $u_{n}, v_{n}, u$ e $v$ são definidos como

$$
\begin{aligned}
& u_{n}(r)=\operatorname{Re} \varphi_{n}(r)=\frac{1}{n} \sum_{j=1}^{n} \cos \left(r^{\prime} X_{j}\right), \\
& v_{n}(r)=\operatorname{Im} \varphi_{n}(r)=\frac{1}{n} \sum_{j=1}^{n} \sin \left(r^{\prime} X_{j}\right), \\
& u(r)=\operatorname{Re} \varphi(r)=E\left[\cos \left(r^{\prime} X_{j}\right)\right], \\
& v(r)=\operatorname{Im} \varphi(r)=E\left[\sin \left(r^{\prime} X_{j}\right)\right]
\end{aligned}
$$

e sendo $R=\left\{r_{1}, \cdots, r_{q}\right\}$ uma malha fixa, onde cada elemento $r_{i} \in \mathbb{R}^{p+1}$. Também podemos escrever

$$
V_{n}-V_{\theta}=\frac{1}{n} \sum_{j=1}^{n} f\left(Z_{j} ; \theta\right),
$$

sendo

$$
f\left(Z_{j} ; \theta\right)=\left(\begin{array}{c}
\cos \left(r_{1}^{\prime} Z_{j}\right)-u\left(r_{1} ; \theta\right) \\
\vdots \\
\cos \left(r_{q}^{\prime} Z_{j}\right)-u\left(r_{q} ; \theta\right) \\
\sin \left(r_{1}^{\prime} Z_{j}\right)-v\left(r_{1} ; \theta\right) \\
\vdots \\
\sin \left(r_{q}^{\prime} Z_{j}\right)-v\left(r_{q} ; \theta\right)
\end{array}\right)_{2 q \times 1}
$$

Podemos notar que estimador DECF pertence à classe dos estimadores extremos, como no caso do estimador CECF. Em particular, este estimador é um estimador do tipo GMM 
(Generalized Method of Moments). Antes de obter as propriedades deste estimador, vamos apresentar a propriedade de convergência do processo $\sqrt{n}\left(V_{n}-V_{\theta}\right)$, que aparece como um lema em Knight e Satchell (1997):

Proposição 5.3 (Convergência $\left.V_{n}-V_{\theta}\right)$ O vetor $\sqrt{n}\left(V_{n}-V_{\theta}\right)$ definido em (5.29) converge em distribuição para

$$
\sqrt{n}\left(V_{n}-V_{\theta}\right) \stackrel{d}{\rightarrow} N_{2 q}\left(0, \Omega_{0}\right)
$$

sendo a matriz $\Omega_{0}$ dada pela seguinte partição

$$
\Omega_{0}=\left[\begin{array}{ll}
\Omega_{R R} & \Omega_{R I} \\
\Omega_{I R} & \Omega_{I I}
\end{array}\right]_{2 q \times 2 q},
$$

e os elementos das matrizes $\Omega_{R R}, \Omega_{R I}, \Omega_{I R}$ e $\Omega_{I I}$ são dados por:

$$
\begin{aligned}
\left\{\Omega_{R R}\right\}_{i, j}= & \frac{1}{2}\left\{\operatorname{Re} \varphi\left(r_{i}-r_{j}\right)+\operatorname{Re} \varphi\left(r_{i}+r_{j}\right)\right\}-n \operatorname{Re} \varphi\left(r_{i}\right) \operatorname{Re} \varphi\left(r_{j}\right)+ \\
& +\frac{1}{2 n} \sum_{k=1}^{n-1}(n-k)\left\{\operatorname{Re} \Lambda_{k}\left(r_{i},-r_{j}\right)+\operatorname{Re} \Lambda_{k}\left(r_{i}, r_{j}\right)+\operatorname{Re} \Lambda_{k}\left(-r_{j}, r_{i}\right)+\operatorname{Re} \Lambda_{k}\left(r_{j}, r_{i}\right)\right\}, \\
\left\{\Omega_{R I}\right\}_{i, j}= & \frac{1}{2}\left\{-\operatorname{Im} \varphi\left(r_{i}-r_{j}\right)+\operatorname{Im} \varphi\left(r_{i}+r_{j}\right)\right\}-n \operatorname{Re} \varphi\left(r_{i}\right) \operatorname{Im} \varphi\left(r_{j}\right)+ \\
& +\frac{1}{2 n} \sum_{k=1}^{n-1}(n-k)\left\{-\operatorname{Im} \Lambda_{k}\left(r_{i},-r_{j}\right)+\operatorname{Im} \Lambda_{k}\left(r_{i}, r_{j}\right)-\operatorname{Im} \Lambda_{k}\left(-r_{j}, r_{i}\right)+\operatorname{Im} \Lambda_{k}\left(r_{j}, r_{i}\right)\right\}, \\
\left\{\Omega_{I R}\right\}_{i, j}= & \frac{1}{2}\left\{\operatorname{Im} \varphi\left(r_{i}-r_{j}\right)+\operatorname{Im} \varphi\left(r_{i}+r_{j}\right)\right\}-n \operatorname{Im} \varphi\left(r_{i}\right) \operatorname{Re} \varphi\left(r_{j}\right)+ \\
& +\frac{1}{2 n} \sum_{k=1}^{n-1}(n-k)\left\{\operatorname{Im} \Lambda_{k}\left(r_{i},-r_{j}\right)+\operatorname{Im} \Lambda_{k}\left(r_{i}, r_{j}\right)+\operatorname{Im} \Lambda_{k}\left(-r_{j}, r_{i}\right)+\operatorname{Im} \Lambda_{k}\left(r_{j}, r_{i}\right)\right\}, \\
& \frac{1}{2}\left\{\operatorname{Re} \varphi\left(r_{i}-r_{j}\right)-\operatorname{Re} \varphi\left(r_{i}+r_{j}\right)\right\}-n \operatorname{Im} \varphi\left(r_{i}\right) \operatorname{Im} \varphi\left(r_{j}\right)+ \\
& +\frac{1}{2 n} \sum_{k=1}^{n-1}(n-k)\left\{\operatorname{Re} \Lambda_{k}\left(r_{i},-r_{j}\right)-\operatorname{Re} \Lambda_{k}\left(r_{i}, r_{j}\right)+\operatorname{Re} \Lambda_{k}\left(-r_{j}, r_{i}\right)-\operatorname{Re} \Lambda_{k}\left(r_{j}, r_{i}\right)\right\} .
\end{aligned}
$$

Prova. Para demonstrar esta proposição basta aplicar a proposição 2.1 para os pares gerados pelo produto cartesiano $\left(r_{1}, \cdots, r_{q}\right) \times\left(r_{1}, \cdots, r_{q}\right)$. Note que $\Omega_{0}=n E\left[\left(V_{n}-V_{\theta}\right)\left(V_{n}-V_{\theta}\right)^{\prime}\right]$.

Teorema 5.1 (Convergência do estimador DECF) Seja $\left\{\widehat{\theta}_{n}\right\}$ a seqüência de estimadores DECF obtida através da otimização em (5.27), de tal forma que $\widehat{\theta}_{n} \stackrel{p}{\rightarrow} \theta_{0}$. Sejam, também, as seguintes hipóteses: 
(a) A seqüência $\left\{Z_{j}\right\}$ é estacionária e $\alpha$-mixing.

(b) A seqüência de matrizes simétricas positivas definidas $W_{n}$ converge em probabilidade para uma matriz simétrica positiva definida $W_{0}, W_{n} \stackrel{p}{\rightarrow} W_{0}$.

(c) A matriz $\frac{\partial^{2} Q_{n}}{\partial \theta \partial \theta^{\prime}}$ e sua inversa existem e são continuas em uma vizinhança aberta $e$ convexa de $\theta_{0}$.

Então, temos:

$$
\left.\frac{\partial^{2} Q_{n}}{\partial \theta \partial \theta^{\prime}}\right|_{\widehat{\theta}_{n}} \stackrel{p}{\rightarrow} B\left(\theta_{0}\right)=\left.\left.2 \frac{\partial V_{\theta}^{\prime}}{\partial \theta}\right|_{\theta_{0}} W_{0} \frac{\partial V_{\theta}}{\partial \theta^{\prime}}\right|_{\theta_{0}},
$$

$e$

$$
\left.\sqrt{n} \frac{\partial Q_{n}}{\partial \theta}\right|_{\theta_{0}} \stackrel{d}{\rightarrow} N\left(0, A\left(\theta_{0}\right)\right)
$$

em que

$$
A\left(\theta_{0}\right)=\lim _{n \rightarrow \infty} E\left[\left.\left.n \frac{\partial Q_{n}}{\partial \theta}\right|_{\theta_{0}} \frac{\partial Q_{n}}{\partial \theta^{\prime}}\right|_{\theta_{0}}\right]=\left.\left.4 \frac{\partial V_{\theta}^{\prime}}{\partial \theta}\right|_{\theta_{0}} W_{0} \Omega_{0} W_{0} \frac{\partial V_{\theta}}{\partial \theta^{\prime}}\right|_{\theta_{0}} .
$$

Além disso, a distribuição limite do estimador DECF $\widehat{\theta}_{n}$ é dada por

$$
\sqrt{n}\left(\widehat{\theta}_{n}-\theta_{0}\right) \stackrel{d}{\longrightarrow} N\left(0, B\left(\theta_{0}\right)^{-1} A\left(\theta_{0}\right) B\left(\theta_{0}\right)^{-1}\right)
$$

sendo

$$
B\left(\theta_{0}\right)^{-1} A\left(\theta_{0}\right) B\left(\theta_{0}\right)^{-1}=\left(\frac{\partial V_{\theta}^{\prime}}{\partial \theta} W_{0} \frac{\partial V_{\theta}}{\partial \theta^{\prime}}\right)^{-1} \frac{\partial V_{\theta}^{\prime}}{\partial \theta} W_{0} \Omega_{0} W_{0} \frac{\partial V_{\theta}}{\partial \theta^{\prime}}\left(\frac{\partial V_{\theta}^{\prime}}{\partial \theta} W_{0} \frac{\partial V_{\theta}}{\partial \theta^{\prime}}\right)^{-1}
$$

Prova. A demonstração deste teorema encontra-se no apêndice E.

O resultado anterior é análogo ao obtido para estimadores GMM, como encontrado, por exemplo, em Hansen (1982) ou Havashi (2000). Note que a eficiência do estimador DECF depende da particular escolha de matrizes de peso $W_{n}$. Podemos calcular uma matriz de pesos de maneira a obter um estimador eficiente no sentido GMM, isto é, que minimiza a matriz de covariância (5.43), através da seguinte proposição (Knight e Satchell (1997)).

Proposição 5.4 (Estimador DECF eficiente GMM) Seja $\Omega_{n}$ um estimador consistente de $\Omega_{0}$, i.e., $\Omega_{n} \stackrel{p}{\rightarrow} \Omega_{0}$. Se tomarmos a matriz de peso $W_{n}=\Omega_{n}^{-1}$, obteremos o estimador DECF eficiente no sentido GMM (isto é, um estimador do tipo DECF com a menor variância em (5.43)), que possui a seguinte distribuição assintótica

$$
\sqrt{n}\left(\widehat{\theta}_{n}-\theta_{0}\right) \stackrel{d}{\longrightarrow} N\left(0,\left(\left.\frac{\partial V_{\theta}^{\prime}}{\partial \theta} \Omega_{0}^{-1} \frac{\partial V_{\theta}}{\partial \theta^{\prime}}\right|_{\theta_{0}}\right)^{-1}\right)
$$

Prova. A demonstração desta proposição encontra-se no apêndice $C$. 
Nas duas últimas seções obtivemos as propriedades dos estimadores CECF e DECF. A única dificuldade remanescente é calcular as derivadas da função característica em relação ao vetor de parâmetros, o que faremos a seguir.

\subsection{Derivadas da função poli-característica em relação ao vetor de parâmetros}

Para obter um estimador para a matriz de covariância assintótica de $\widehat{\theta}$ precisamos obter as derivadas da função poli-característica $\varphi_{z}$ em relação ao vetor de parâmetros $\theta=\left(\delta, \phi, \sigma_{\eta}, \lambda\right)^{\prime}$. Lembrando que $\varphi_{z}$ é dada por:

$$
\begin{aligned}
\varphi_{z}(r)= & \exp \left\{\frac{i \delta}{1-\phi} \sum_{\zeta=1}^{p+1} r_{\zeta}-\frac{\sigma_{\eta}^{2}}{2\left(1-\phi^{2}\right)}\left(\sum_{\zeta=1}^{p+1} \phi^{\zeta-1} r_{\zeta}\right)^{2}-\right. \\
& \left.-\sum_{j=2}^{p+1} \frac{\sigma_{\eta}^{2}}{2}\left(\sum_{\zeta=j}^{p+1} \phi^{\zeta-j} r_{\zeta}\right)^{2}\right\} \prod_{j=1}^{p+1} \varphi_{\varepsilon}\left(r_{j}\right),
\end{aligned}
$$

em que

$$
\begin{gathered}
\varphi_{\psi}(t)=\exp \left(\frac{i t \delta}{1-\phi}-\frac{\sigma_{\eta}^{2} t^{2}}{2\left(1-\phi^{2}\right)}\right), \\
\varphi_{\eta}(t)=\exp \left(-\frac{t^{2}}{2}\right), \\
\varphi_{\varepsilon}(t)=\left\{\begin{array}{cc}
\Gamma(1+i t) & , \text { se exponencial } \\
\frac{\Gamma\left(1+i t \lambda^{-1}\right)}{\left[\Gamma\left(1+\lambda^{-1}\right)\right]^{i t}} & , \text { se Weibull } \\
\frac{\Gamma(\lambda+i t)}{\lambda^{i t} \Gamma(\lambda)} & \text {, se gama. }
\end{array}\right.
\end{gathered}
$$

Nas derivadas a seguir vamos utilizar a função digama definida por:

$$
\psi(z)=\frac{\Gamma^{\prime}(z)}{\Gamma(z)} .
$$

Antes de calcular as derivadas da função poli-característica $\varphi_{z}$, vamos calcular as derivadas da função característica dos log-erros $\varphi_{\varepsilon}$.

Proposição 5.5 (Derivadas parciais de $\varphi_{\varepsilon}$ ) As derivadas da função característica dos log-erros, $\varphi_{\varepsilon}(r)$, em relação a $\lambda$ (parâmetro do modelo $S C D$ ) são dadas por:

\section{- Exponencial:}

$$
\frac{\partial \varphi_{\varepsilon}(r)}{\partial \lambda}=0
$$


-Weibull:

$$
\frac{\partial \varphi_{\varepsilon}(r)}{\partial \lambda}=\frac{i t}{\lambda^{2}} \frac{\Gamma\left(1+i r \lambda^{-1}\right)}{\left[\Gamma\left(1+\lambda^{-1}\right)\right]^{i t}}\left\{-\psi\left(1+i r \lambda^{-1}\right)+\psi\left(1+\lambda^{-1}\right)\right\}
$$

- Gama:

$$
\frac{\partial \varphi_{\varepsilon}(r)}{\partial \lambda}=\frac{1}{\lambda^{i t}} \frac{\Gamma(\lambda+i r)}{\Gamma(\lambda)}\left\{\psi(\lambda+i r)-\frac{i r}{\lambda}-\psi(\lambda)\right\}
$$

Prova. A demonstração desta proposição encontra-se no apêndice C.

Proposição 5.6 (Derivadas parciais de $\varphi_{z}$ ) As derivadas parciais da função policaracterística, $\varphi_{z}(r)$, em relação aos parâmetros do modelo $S C D, \theta=\left(\delta, \phi, \sigma_{\eta}, \lambda\right)^{\prime}$, são dadas por

$$
\begin{aligned}
& \frac{\partial \varphi_{z}(r)}{\partial \delta}=\varphi_{z}(r)\left(\frac{i}{1-\phi} \sum_{\zeta=1}^{p+1} r_{\zeta}\right) \\
& \frac{\partial \varphi_{z}(r)}{\partial \phi}=\varphi_{z}(r)\left\{\frac{i \delta}{(1-\phi)^{2}} \sum_{\zeta=1}^{p+1} r_{\zeta}-\frac{\sigma_{\eta}^{2} \phi}{\left(1-\phi^{2}\right)^{2}}\left(\sum_{\zeta=1}^{p+1} \phi^{\zeta-1} r_{\zeta}\right)^{2}-\right. \\
& -\frac{\sigma_{\eta}^{2}}{\left(1-\phi^{2}\right)}\left(\sum_{\zeta=1}^{p+1} \phi^{\zeta-1} r_{\zeta}\right)\left(\sum_{\zeta=1}^{p+1}(\zeta-1) \phi^{\zeta-2} r_{\zeta}\right)- \\
& \left.-\sum_{j=2}^{p+1} \sigma_{\eta}^{2}\left(\sum_{\zeta=j}^{p+1} \phi^{\zeta-j} r_{\zeta}\right)\left(\sum_{\zeta=j}^{p+1}(\zeta-j) \phi^{\zeta-j-1} r_{\zeta}\right)\right\} \\
& \frac{\partial \varphi_{z}(r)}{\partial \sigma_{\eta}}=\varphi_{z}(r)\left\{-\frac{\sigma_{\eta}}{\left(1-\phi^{2}\right)}\left(\sum_{\zeta=1}^{p+1} \phi^{\zeta-1} r_{\zeta}\right)^{2}-\sum_{j=2}^{p+1} \sigma_{\eta}\left(\sum_{\zeta=j}^{p+1} \phi^{\zeta-j} r_{\zeta}\right)^{2}\right\} \\
& \frac{\partial \varphi_{z}(r)}{\partial \lambda}=\varphi_{z}(r) \sum_{j=1}^{p+1} \frac{1}{\varphi_{\varepsilon}\left(r_{j}\right)} \frac{\partial \varphi_{\varepsilon}\left(r_{j}\right)}{\partial \lambda} .
\end{aligned}
$$

Prova. A demonstração desta proposição encontra-se no apêndice $C$.

\subsection{Restrições impostas pelo espaço paramétrico}

A otimização em (5.1) ou (5.27) está sujeita às restrições impostas pelo espaço paramétrico $\Theta$. Para o nosso caso temos que $\theta=\left(\delta, \phi, \sigma_{\eta}, \lambda\right)^{\prime}$, com as restrições $\sigma_{\eta}>0$ e $-1<\phi<1$ (para que o processo seja estacionário). Mas como a otimização sujeita à restrições é um problema notoriamente difícil, é conveniente transformar as variáveis de maneira a eliminar as restrições. Em particular, vamos empregar as seguintes transformações: 


$$
\sigma_{\eta}=e^{\zeta},-\infty<\zeta<\infty
$$

e

$$
\phi=\frac{\chi}{\sqrt{1+\chi^{2}}},-\infty<\chi<\infty .
$$

De uma maneira geral podemos denotar o vetor de transformações por $\theta=g(\xi)$. Para o nosso caso se denotarmos o vetor de parâmetros transformados de $\xi=(\delta, \chi, \zeta, \lambda)^{\prime}$ podemos escrever

$$
\theta=g(\xi)=\left(\begin{array}{c}
\delta \\
\frac{\chi}{\sqrt{1+\chi^{2}}} \\
e^{\zeta} \\
\lambda
\end{array}\right)_{4 \times 1}
$$

Neste caso, a otimização (5.1) ou (5.27) pode ser efetuada utilizando-se um algoritmo de busca irrestrita para a solução do problema

$$
\widehat{\xi}_{n}=\arg \min _{\xi \in \mathbb{R}^{4}} Q_{n}(g(\xi)),
$$

obtendo-se $\widehat{\theta}_{n}=g\left(\widehat{\xi}_{n}\right)$.

\subsection{Sumário}

Neste capítulo vimos como utilizar a função poli-característica do modelo SCD para sua estimação através dos métodos CECF e DECF, revendo também os resultados de convergência assintótica destes estimadores. Também obtivemos as derivadas da função poli-característica, que são utilizadas na estimação das matrizes de covariância dos estimadores.

No próximo capítulo vamos utilizar modelos de espaço de estados para estimação das variáveis latentes $\left(\psi_{j}\right)$. Em particular, vamos utilizar uma aproximação Gaussiana para o modelo SCD, permitindo o uso do chamado filtro de Kalman. Este algoritmo permite a estimação dos parâmetros do modelo pela maximização da verossimilhança "aproximada" (já que o verdadeiro modelo é não Gaussiano), resultando no método da "quase-verossimilhança" (QML - Quasi Maximum Likelihood). 



\section{Capítulo 6}

\section{Estimação das variáveis latentes no modelo SCD}

Nos capítulos anteriores vimos como utilizar o método da função característica empírica para eliminar a variável latente e, assim, estimarmos os parâmetros do modelo SCD. Entretanto, muitas vezes estamos interessados justamente na variável latente (é o caso, também, do modelo de volatilidade estocástica).

Para obter estimativas das variáveis latentes é necessário inicialmente colocar o modelo SCD no formato de modelo de espaço de estados, como vimos no Capítulo 4 .

$$
\begin{gathered}
\psi_{t}=\delta+\phi \psi_{t-1}+\sigma_{\eta} \eta_{t}, \\
y_{t}=\psi_{t}+\varepsilon_{t}
\end{gathered}
$$

em que (6.1) é a equação de estado ou transição, (6.2) é a equação de observação ou de mensuração e $y_{t}$ é o logaritmo da duração.

Vamos adotar a seguinte notação $y_{1: t}=\left\{y_{1}, \cdots, y_{t}\right\}$ para indicar o vetor de observações disponíveis até o instante $t$. A análise de espaço de estados busca obter estimativas para a variável latente $\psi_{t}$, dado o vetor de observações $y_{1: s}$. Quando $s<t$ o problema é chamado previsão. Quando $s=t$ temos o problema de filtragem. Finalmente, quando $s>t$ temos o problema de suavização.

Uma revisão sobre modelos de espaço de estados não Gaussianos pode ser encontrada, por exemplo, em Fearnhead (1998), Durbin e Koopman (2000) e Briers et al. (2004). Neste capítulo vamos utilizar dois métodos propostos na literatura para obter estimativas para as variáveis latentes:

- Solução Bayesiana através de integração numérica;

- Filtro de Kalman, utilizado por Bauwens e Veredas (2004).

Além dos dois métodos acima, vamos desenvolver um algoritmo recursivo, baseado na expansão de Gram-Charlier da densidade dos erros ("filtro Gram-Charlier"). 
Na seção abaixo vamos rever a relações recursivas de previsão e filtragem para o modelo de espaço de estados, como desenvolvido por Kitagawa (1987). A seguir vamos rever o filtro de Kalman como aproximação para o modelo SCD. Finalmente, vamos apresentar o "filtro de Gram-Charlier".

\subsection{Relações recursivas de previsão e filtragem}

\subsubsection{Previsão um passo adiante (atualização do tempo)}

Vamos assumir que estejamos em $t-1$, e que possuamos a distribuição condicional da variável latente neste momento dadas as observações disponíveis, $\psi_{t-1} \mid y_{1: t-1}$. A previsão um passo adiante para a variável latente pode ser obtida a partir da distribuição conjunta de $\psi_{t}$ e $\psi_{t-1}$ integrando-se $\psi_{t-1}$ :

$$
\begin{gathered}
p_{\theta}\left(\psi_{t} \mid y_{1: t-1}\right)=\int p_{\theta}\left(\psi_{t}, \psi_{t-1} \mid y_{1: t-1}\right) d \psi_{t-1} \\
p_{\theta}\left(\psi_{t} \mid y_{1: t-1}\right)=\int p_{\theta}\left(\psi_{t} \mid \psi_{t-1}\right) p_{\theta}\left(\psi_{t-1} \mid y_{1: t-1}\right) d \psi_{t-1}
\end{gathered}
$$

\subsubsection{Filtro (atualização após observação)}

Após a observação dos dados no instante $t, y_{t}$, atualizamos a estimativa da variável latente através do método Bayesiano, sendo a priori dada pela equação de previsão um passo adiante de $t-1(\underline{6.3})$ e sendo $p_{\theta}\left(y_{t} \mid \psi_{t}\right)$ a densidade condicional dos dados:

$$
\begin{aligned}
p_{\theta}\left(\psi_{t} \mid y_{1: t}\right) & =p_{\theta}\left(\psi_{t} \mid y_{t}, y_{1: t-1}\right) \\
& =\frac{p_{\theta}\left(\psi_{t}, y_{t} \mid y_{1: t-1}\right)}{p_{\theta}\left(y_{t} \mid y_{1: t-1}\right)} \\
& =\frac{p_{\theta}\left(y_{t} \mid \psi_{t}\right) p_{\theta}\left(\psi_{t} \mid y_{1: t-1}\right)}{p_{\theta}\left(y_{t} \mid y_{1: t-1}\right)} \\
p_{\theta}\left(\psi_{t} \mid y_{1: t}\right) & =c^{-1} p_{\theta}\left(y_{t} \mid \psi_{t}\right) p_{\theta}\left(\psi_{t} \mid y_{1: t-1}\right)
\end{aligned}
$$

onde,

$$
c=p_{\theta}\left(y_{t} \mid y_{1: t-1}\right)=\int p_{\theta}\left(y_{t} \mid \psi_{t}\right) p_{\theta}\left(\psi_{t} \mid y_{1: t-1}\right) d \psi_{t}
$$




\subsubsection{Suavização}

Para obter a densidade após a observação de todos os dados (suavização), vamos inicialmente considerar a expressão da densidade conjunta para $\psi_{t}$ e $\psi_{t+1}$, dada a seqüência de dados $y_{1: T}$ :

$$
\begin{aligned}
p_{\theta}\left(\psi_{t}, \psi_{t+1} \mid y_{1: T}\right) & =p_{\theta}\left(\psi_{t+1} \mid y_{1: T}\right) p_{\theta}\left(\psi_{t} \mid \psi_{t+1}, y_{1: T}\right) \\
& =p_{\theta}\left(\psi_{t+1} \mid y_{1: T}\right) p_{\theta}\left(\psi_{t} \mid \psi_{t+1}, y_{1: t}\right) \\
& =p_{\theta}\left(\psi_{t+1} \mid y_{1: T}\right) \frac{p_{\theta}\left(\psi_{t}, \psi_{t+1} \mid y_{1: t}\right)}{p_{\theta}\left(\psi_{t+1} \mid y_{1: t}\right)} \\
& =\frac{p_{\theta}\left(\psi_{t+1} \mid y_{1: T}\right) p_{\theta}\left(\psi_{t+1} \mid \psi_{t}\right) p_{\theta}\left(\psi_{t} \mid y_{1: t}\right)}{p_{\theta}\left(\psi_{t+1} \mid y_{1: t}\right)}
\end{aligned}
$$

Obtemos a densidade suavizada para a variável latente $\psi_{t}$ integrando-se (ㅎ․6) em $\psi_{t+1}$ :

$$
\begin{aligned}
p_{\theta}\left(\psi_{t} \mid y_{1: T}\right) & =\int p_{\theta}\left(\psi_{t}, \psi_{t+1} \mid y_{1: T}\right) d \psi_{t+1} \\
& =\int \frac{p_{\theta}\left(\psi_{t+1} \mid y_{1: T}\right) p_{\theta}\left(\psi_{t+1} \mid \psi_{t}\right) p_{\theta}\left(\psi_{t} \mid y_{1: t}\right)}{p_{\theta}\left(\psi_{t+1} \mid y_{1: t}\right)} d \psi_{t+1} \\
p_{\theta}\left(\psi_{t} \mid y_{1: T}\right) & =p_{\theta}\left(\psi_{t} \mid y_{1: t}\right) \int \frac{p_{\theta}\left(\psi_{t+1} \mid y_{1: T}\right) p_{\theta}\left(\psi_{t+1} \mid \psi_{t}\right)}{p_{\theta}\left(\psi_{t+1} \mid y_{1: t}\right)} d \psi_{t+1}
\end{aligned}
$$

Geralmente não é possível efetuar analiticamente as integrais em (6.5) a (6.7) e assim temos que recorrer a algum método de integração numérica. Em particular, podemos utilizar a já mencionada quadratura de Gauss-Hermite, que será utilizada no capítulo seguinte para testar o algoritmo.

Caso as distribuições condicionais envolvidas nas integrais em (6.5) a (6.7) permitam sua integração analítica, pode-se obter explicitamente as funções densidade de probabilidade para a variável latente. Em particular, quando as distribuições de probabilidade associadas com (4.91) e (4.92) são Gaussianas, as densidades das variáveis latentes, também, são distribuições normais, o que permite o uso do filtro de Kalman (ver, por exemplo, Brockwell e Davis (1991), Shumway e Stoffer (2000) ou Lütkepohl (2005)). A estrutura Gaussiana do filtro de Kalman permite que estas distribuições sejam caracterizadas por apenas dois momentos: a média e a covariância. A seguir vamos particularizar a solução do filtro de Kalman para o modelo SCD.

\subsection{Filtro e suavizador de Kalman}

O filtro de Kalman é um algoritmo recursivo utilizado para estimar as variáveis latentes de um modelo de espaço de estados quando as equações de mensuração e de observação são lineares e os choques dessas equações são normais (Gaussianos). Para obter as relações de recursão do filtro de Kalman para o modelo SCD vamos utilizar a seguinte notação, adaptada 
de Lütkepohl (2005):

$$
\begin{aligned}
\psi_{t \mid s} & :=E\left[\psi_{t} \mid y_{1: s}\right], \\
\sigma_{\psi}^{2}(t \mid s) & :=\operatorname{Cov}\left[\psi_{t} \mid y_{1: s}\right], \\
y_{t \mid s} & :=E\left[y_{t} \mid y_{1: s}\right], \\
\sigma_{y}^{2}(t \mid s) & :=\operatorname{Cov}\left[y_{t} \mid y_{1: s}\right] .
\end{aligned}
$$

A solução genérica do filtro de Kalman pode ser encontrada, por exemplo, em Brockwell e Davis (1991), Shumway e Stoffer (2000) ou Lütkepohl (2005). Particularizando o filtro de Kalman para o nosso problema, como posto pelas equações (4.91) e (4.92), obtemos a seguinte solução:

Proposição 6.1 (Filtro de Kalman para o modelo SCD) Seja o modelo no formato de espaço de estados definido pelas equações (6.1)e (6.2). Se assumirmos que o choque aleatório $\varepsilon_{t}$ seja independentemente e identicamente normalmente distribuido

$$
\varepsilon_{t} \sim N I I D\left(\mu_{\varepsilon}, \sigma_{\varepsilon}^{2}\right)
$$

então as relações de recursão do filtro de Kalman são dadas por:

- Previsão um passo adiante (atualização do tempo, $1 \leq t \leq T$ )

$$
\begin{gathered}
\psi_{t \mid t-1}=\delta+\phi \psi_{t-1 \mid t-1}, \\
\sigma_{\psi}^{2}(t \mid t-1)=\phi^{2} \sigma_{\psi}^{2}(t-1 \mid t-1)+\sigma_{\eta}^{2}, \\
y_{t \mid t-1}=\psi_{t \mid t-1}+\mu_{\varepsilon}, \\
\sigma_{y}^{2}(t \mid t-1)=\sigma_{\psi}^{2}(t \mid t-1)+\sigma_{\varepsilon}^{2},
\end{gathered}
$$

sendo que $\mu_{\varepsilon}=E\left[\varepsilon_{t}\right]$ e $\sigma_{\varepsilon}^{2}=\operatorname{Var}\left[\varepsilon_{t}\right]$ são dados pelos resultados da proposição 4.11 .

- Filtragem (atualização após observação, $1 \leq t \leq T$ )

$$
\begin{gathered}
\psi_{t \mid t}=\psi_{t \mid t-1}+P_{t}\left(y_{t}-y_{t \mid t-1}\right), \\
\sigma_{\psi}^{2}(t \mid t)=\sigma_{\psi}^{2}(t \mid t-1)-P_{t}^{2} \sigma_{y}^{2}(t \mid t-1),
\end{gathered}
$$

em que

$$
P_{t}=\frac{\sigma_{\psi}^{2}(t \mid t-1)}{\sigma_{y}^{2}(t \mid t-1)}
$$

- Suavização $(t<T)$

$$
\begin{gathered}
\psi_{t \mid T}=\psi_{t \mid t}+S_{t}\left(\psi_{t+1 \mid T}-\psi_{t+1 \mid t}\right) \\
\sigma_{\psi}^{2}(t \mid T)=\sigma_{\psi}^{2}(t \mid t)-S_{t}^{2}\left[\sigma_{\psi}^{2}(t+1 \mid t)-\sigma_{\psi}^{2}(t+1 \mid T)\right]
\end{gathered}
$$




$$
S_{t}=\phi \frac{\sigma_{\psi}^{2}(t \mid t)}{\sigma_{\psi}^{2}(t+1 \mid t)}
$$

Prova. A prova desta proposição consiste simplesmente em aplicar os resultados de Lütkepohi (2005), pg. 626 a 630, ao modelo de espaço de estados definido por (6.1) e (6.2), tomando-se apenas o cuidado de notar que a variável $\varepsilon_{t}$ tem média $\mu_{\varepsilon}$ diferente de 0.

É importante notar que no nosso presente caso, a distribuição de $\varepsilon_{t}$ é não Gaussiana, de forma que o uso do filtro de Kalman é apenas uma aproximação para o problema de estimação do vetor de estado. As grandes vantagens deste filtro são a sua solução analítica, o que permite computação extremamente rápida, e a possibilidade de escrever a função de verossimilhança explicitamente.

\subsubsection{Estimativa por quase-verossimilhança (QML)}

Como as distribuições dos log-erros não são Gaussianas, a verossimilhança obtida ao utilizarse o filtro de Kalman é apenas uma aproximação da verdadeira verossimilhança. Por isso, a obtenção de estimadores pela maximização da verossimilhança com o filtro de Kalman é conhecida como estimativa de "quase-verossimilhança" (QML - Quasi-Maximum Likelihood).

Podemos escrever a função de log-verossimilhança para o modelo SCD através da seguinte proposição:

Proposição 6.2 (Quase-verossimilhança para o modelo SCD) Para o modelo de espaço de estados (6.1) a (6.2) temos que a função de verossimilhança é dada por

$$
\ln l(\theta)=-\frac{T}{2} \ln (2 \pi)-\frac{1}{2} \sum_{t=1}^{T}\left[\ln \sigma_{t}^{2}(\theta)+\frac{e_{t}^{2}(\theta)}{\sigma_{t}^{2}(\theta)}\right],
$$

em que explicitamos a dependência de $e_{t}(\theta)$ e $\sigma_{t}^{2}(\theta)$ do vetor de parâmetros $\theta$, sendo que

$$
\begin{gathered}
e_{t}(\theta)=y_{t}-y_{t \mid t-1}, \\
\sigma_{t}^{2}(\theta)=\sigma_{y}^{2}(t \mid t-1),
\end{gathered}
$$

e $y_{t \mid t-1}$ e $\sigma_{y}^{2}(t \mid t-1)$ são obtidos através da proposição 6.1.

Prova. Para demonstrar a proposição acima, basta aplicar o resultado de Lütkepohi (2005), pg.633, ao modelo SCD.

A matriz de informação associada à função de verossimilhança do filtro de Kalman é dada em Lütkepohl (2005), pg. 635, que adaptada ao modelo SCD fornece a seguinte proposição:

Proposição 6.3 (Matriz de informação do filtro de Kalman para o modelo SCD) Para o modelo de espaço de estados (6.1) a (6.2) temos que a matriz de informação é dada 
por

$$
\begin{aligned}
I\left(\theta_{0}\right) & =-E\left[\left.\frac{\partial^{2} \ln l}{\partial \theta \partial \theta^{\prime}}\right|_{\theta_{0}}\right] \\
& =\frac{1}{2} \sum_{t=1}^{T}\left\{\frac{\partial \sigma_{t}^{2 \prime}}{\partial \theta} \frac{\partial \sigma_{t}^{2}}{\partial \theta^{\prime}} \frac{1}{\left(\sigma_{t}^{2}\right)^{2}}+2 E\left[\frac{\partial e_{t}^{\prime}}{\partial \theta} \frac{\partial e_{t}}{\partial \theta^{\prime}} \frac{1}{\sigma_{t}^{2}}\right]\right\},
\end{aligned}
$$

sendo que $e_{t}(\theta)$ e $\sigma_{t}(\theta)$ são obtidos através da proposição 6.2.

Prova. Para demonstrar a proposição acima, basta aplicar o resultado de Lütkepohi (2005), pg.635, ao modelo SCD.

Naturalmente como o verdadeiro parâmetro $\theta_{0}$ envolvido na proposição acima é desconhecido, podemos obter uma estimativa da matriz de informação utilizando seu estimador e desprezando o operador valor esperado na expressão acima. Sob o conjunto de hipóteses explicitado em Lütkepohl (2005), pg. 636, o estimador de máxima verossimilhança $\theta_{n}$ converge em distribuição para uma distribuição normal

$$
\sqrt{n}\left(\theta_{n}-\theta_{0}\right) \stackrel{d}{\rightarrow} N\left(0, \Sigma_{\theta_{n}}\right)
$$

em que

$$
\Sigma_{\theta_{n}}=\lim _{n \rightarrow \infty} n I\left(\theta_{0}\right)^{-1}
$$

\subsection{Procedimento recursivo baseado na expansão de Gram-Charlier ("filtro Gram-Charlier")}

Como o filtro de Kalman só utiliza os dois primeiros momentos, vamos apresentar agora um método para utilizar os quatro primeiros momentos - ao invés de apenas os dois primeiros - com a expectativa de obter um estimador mais robusto para distribuições "distantes" da normal (com assimetria e excesso de curtose). Mas para isso, precisamos obter uma aproximação para a densidade, dados os momentos. Uma abordagem natural seria utilizar uma aproximação da densidade não Gaussiana por alguma expansão em séries de polinômios ortogonais (para facilitar as integrais envolvidas), tais como a expansão de Gram-Charlier ou Edgeworth. Sorenson e Stubberud (1968) parecem ter sidos os primeiros a desenvolver um procedimento recursivo baseado na expansão de Edgeworth, truncada até quarta ordem ${ }^{1}$, enquanto Srinivasan (1970) emprega a expansão de Gram-Charlier no seu filtro recursivo. Mais recentemente Challa et al. (2000) utilizam um procedimento baseado em uma expansão "generalizada" de Edgeworth em conjunto com quadratura de Gauss-Hermite. Aqui neste trabalho, entretanto, vamos utilizar a expansão de Gram-Charlier até quarta-ordem pelos motivos que explicaremos logo a seguir. Esta expansão permite aproximar a densidade de

${ }^{1}$ Sorenson (1988) indica também a referência abaixo para filtros não gaussianos utilizando expansão de Edgeworth, que infelizmente não conseguimos obter:

Kizner, W., Optimal nonlinear estimation based on orthogonal expansions, Technical Report 32-1366, Jet Propulsion Laboratory, Pasadena, California, 1969. 
uma variável aleatória $X$ usando os quatro primeiros momentos por (veja, por exemplo, Stuart e Ord $(\underline{1994}))^{2}$ :

$$
f(x) \approx \frac{z(w)}{\sigma}\left\{1+\frac{\gamma_{1}}{6}\left(w^{3}-3 w\right)+\frac{\gamma_{2}}{24}\left(w^{4}-6 w^{2}+3\right)\right\}
$$

onde, $\quad w=\frac{x-\mu}{\sigma}, z(w)=\frac{e^{-w^{2} / 2}}{\sqrt{2 \pi}}$ é a densidade da normal padrão $(0,1), \gamma_{1}=\mu_{3} / \sigma^{3}$, $\gamma_{2}=\mu_{4} / \sigma^{4}-3, \sigma=\sqrt{\mu_{2}}$ e $\mu_{j}=E\left[(X-\mu)^{j}\right]$.

As aproximações sugeridas são formadas por séries de polinômios; assim, elas costumam ter um comportamento ruim nas caudas e não constituem densidades, a não ser que sejam impostas restrições no espaço paramétrico (Barton e Dennis (1952) e Draper e Tierney (1972)). Como Jondeau e Rockinger (1999) já obtiveram estas restrições para o caso da expansão de Gram-Charlier, vamos utilizar esta expansão ao invés da expansão de Edgeworth ${ }^{3}$. A figura (6.1) ilustra a aproximação de Gram-Charlier até o $4^{\circ}$ momento para as distribuições do modelo SCD. Note pelas figuras à esquerda que, sem a imposição de restrições no espaço paramétrico para $\left(\gamma_{1}, \gamma_{2}\right)$, as aproximações não constituem funções densidade de probabilidade. Já as figuras à direita ilustram o efeito da aplicação das restrições derivadas por Jondeau e Rockinger (1999) (que explicitaremos em uma subseção a seguir).

${ }^{2} \mathrm{~A}$ expansão de Edgeworth até $4^{a}$ ordem contém um termo adicional:

$$
f(x) \approx \frac{z(w)}{\sigma}\left\{1+\frac{\gamma_{1}}{6}\left(w^{3}-3 w\right)+\frac{\gamma_{2}}{24}\left(w^{4}-6 w^{2}+3\right)+\frac{\gamma_{1}^{2}}{72}\left(w^{6}-15 w^{4}+45 w^{2}-15\right)\right\} .
$$

${ }^{3}$ Notamos entretanto que Balitskaya e Zolotuhina (1988) obtiveram as condições para assimetria e curtose para que uma expansão em série de Edgeworth truncada possa constituir uma densidade em um intervalo finito. 

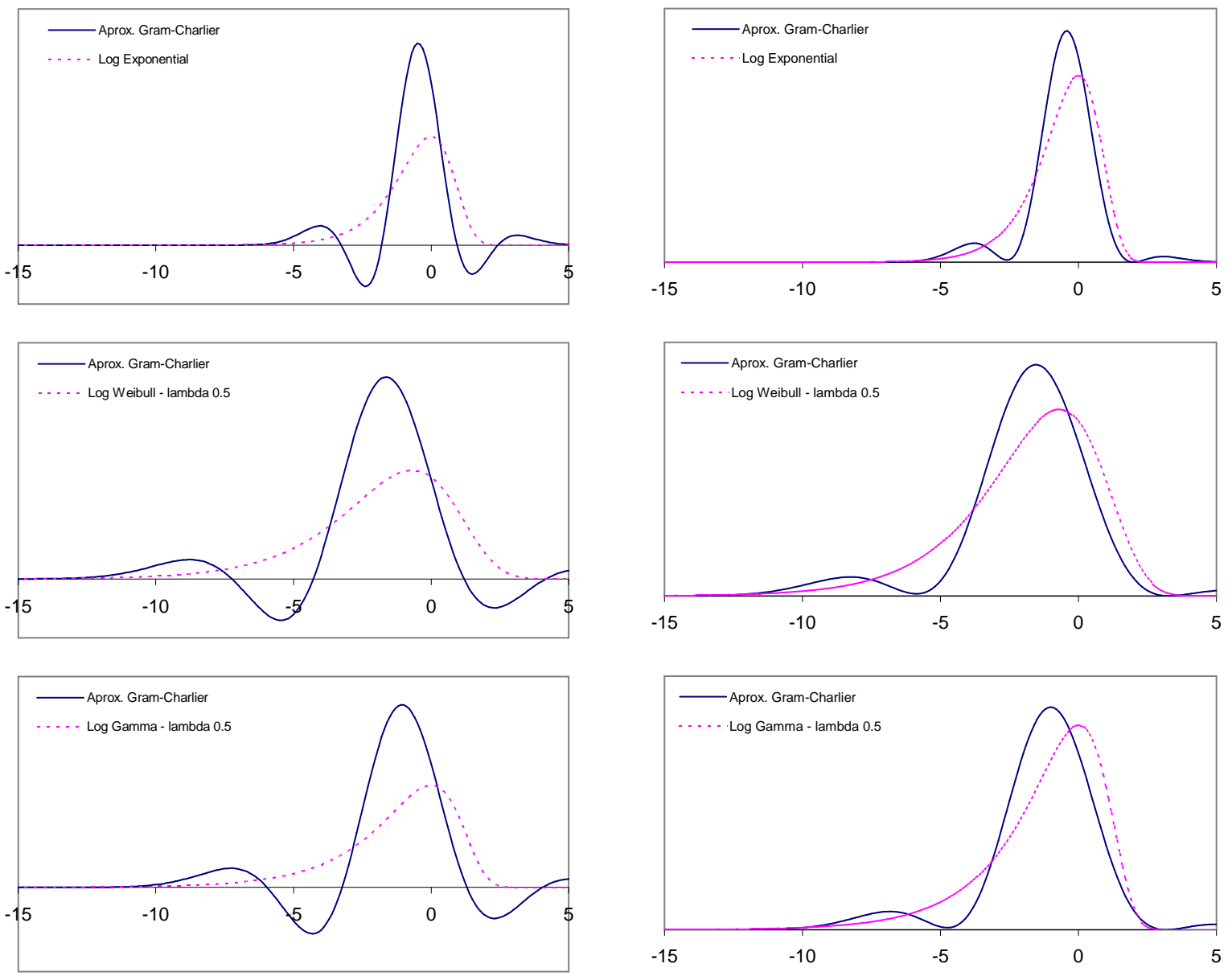

Figura 6.1: Aproximação de Gram-Charlier para a densidade, sem imposição de restrições (à esquerda) e com imposição de restrições nos parâmetros $\left(\gamma_{1}, \gamma_{2}\right)$ conforme Jondeau e Rockinger (1999) (à direita), para as seguintes distribuições: log-exponencial (4.38) em cima, log-Weibull (4.39), $\lambda=0.5$, no meio, e log-gama (4.40), $\lambda=0.5$, em baixo. 
Seja então o seguinte modelo de espaço de estados univariado:

$$
\begin{gathered}
X_{t}=\delta+\phi X_{t-1}+\eta_{t}, \\
Y_{t}=\alpha+\beta X_{t}+\varepsilon_{t}, \\
\eta_{t} \sim \operatorname{NIID}\left(0, \sigma_{\eta}^{2}\right), \\
\varepsilon_{t} \sim \text { alguma distribuição i.i.d. com momentos finitos até } 4^{a} \text { ordem. }
\end{gathered}
$$

Adotaremos a seguinte notação, análoga à empregada em Shumway e Stoffer (2000):

$$
\begin{aligned}
\mu_{t}^{s} & =E\left[X_{t} \mid y_{1: s}\right], \\
v_{t}^{s} & =\left(\sigma_{t}^{s}\right)^{2}=E\left[\left(X_{t}-\mu_{t}^{s}\right)^{2} \mid y_{1: s}\right], \\
\gamma_{1, t}^{s} & =\frac{E\left[\left(X_{t}-\mu_{t}^{s}\right)^{3} \mid y_{1: s}\right]}{\left(\sigma_{t}^{s}\right)^{3}}, \\
\gamma_{2, t}^{s} & =\frac{E\left[\left(X_{t}-\mu_{t}^{s}\right)^{4} \mid y_{1: s}\right]}{\left(\sigma_{t}^{s}\right)^{4}}-3,
\end{aligned}
$$

Proposição 6.4 (Filtro Gram-Charlier) Seja o modelo de espaço de estados definido por (6.22) a 6.25). Neste caso temos

- Previsão um passo adiante (atualização do tempo): a distribuição da variável latente, $X_{t} \mid y_{1: t-1}$, pode ser aproximada por

$$
p\left(x_{t} \mid y_{1: t-1}\right) \cong \frac{e^{-\frac{1}{2} \zeta_{t}^{2}}}{\sqrt{2 \pi} \sigma_{t}^{t-1}}\left\{1+\frac{\gamma_{1, t}^{t-1}}{6}\left(\zeta_{t}^{3}-3 \zeta_{t}\right)+\frac{\gamma_{2, t}^{t-1}}{24}\left(\zeta_{t}^{4}-6 \zeta_{t}^{2}+3\right)\right\}
$$

onde

$$
\begin{aligned}
\mu_{t}^{t-1} & =\delta+\phi \mu_{t-1}^{t-1}, \\
v_{t}^{t-1} & =\left(\sigma_{t}^{t-1}\right)^{2}=\phi^{2} v_{t-1}^{t-1}+\sigma_{\eta}^{2}, \\
\gamma_{1, t}^{t-1} & =\gamma_{1, t-1}^{t-1}\left(\frac{\phi \sigma_{t-1}^{t-1}}{\sqrt{\phi^{2} v_{t-1}^{t-1}+\sigma_{\eta}^{2}}}\right)^{3}=\gamma_{1, t-1}^{t-1}\left(\frac{\phi \sigma_{t-1}^{t-1}}{\sigma_{t}^{t-1}}\right)^{3}, \\
\gamma_{2, t}^{t-1} & =\gamma_{2, t-1}^{t-1}\left(\frac{\phi \sigma_{t-1}^{t-1}}{\sqrt{\phi^{2} v_{t-1}^{t-1}+\sigma_{\eta}^{2}}}\right)^{4}=\gamma_{2, t-1}^{t-1}\left(\frac{\phi \sigma_{t-1}^{t-1}}{\sigma_{t}^{t-1}}\right)^{4}, \\
\zeta_{t} & =\frac{x_{t}-\delta-\phi \mu_{t-1}^{t-1}}{\sqrt{\phi^{2} v_{t-1}^{t-1}+\sigma_{\eta}^{2}}}=\frac{x_{t}-\mu_{t}^{t-1}}{\sigma_{t}^{t-1}} .
\end{aligned}
$$

- Filtragem (atualização após observação): a distribuição da variável latente após a 
observação, $X_{t} \mid y_{1: t}$, pode ser aproximada por:

$$
p\left(x_{t} \mid y_{1: t}\right) \cong \frac{e^{-\frac{1}{2} \xi_{t}^{2}}}{\sqrt{2 \pi} \sigma_{t}^{t}}\left\{1+\frac{\gamma_{1, t}^{t}}{6}\left(\xi_{t}^{3}-3 \xi_{t}\right)+\frac{\gamma_{2, t}^{t}}{24}\left(\xi_{t}^{4}-6 \xi_{t}^{2}+3\right)\right\}
$$

onde

$$
\begin{aligned}
\xi_{t} & =\frac{x_{t}-\mu_{t}^{t}}{\sigma_{t}^{t}}, \\
\mu_{t}^{t} & =\mu_{t}^{t-1}+\sigma_{t}^{t-1} \frac{g_{1}}{g_{0}} \\
v_{t}^{t} & =\left(\sigma_{t}^{t}\right)^{2}=v_{t}^{t-1}\left[\frac{g_{2}}{g_{0}}-\left(\frac{g_{1}}{g_{0}}\right)^{2}\right], \\
\gamma_{1, t}^{t} & =\frac{\mu_{3}}{\left(\sigma_{t}^{t}\right)^{3}}, \\
\gamma_{2, t}^{t} & =\frac{\mu_{4}}{\left(\sigma_{t}^{t}\right)^{4}}-3,
\end{aligned}
$$

sendo que

$$
\begin{aligned}
& \mu_{2}=\mu_{2}^{\prime}-\mu_{1}^{\prime 2}, \\
& \mu_{3}=\mu_{3}^{\prime}-3 \mu_{2}^{\prime} \mu_{1}^{\prime}+2 \mu_{1}^{\prime 3}, \\
& \mu_{4}=\mu_{4}^{\prime}-4 \mu_{3}^{\prime} \mu_{1}^{\prime}+6 \mu_{2}^{\prime} \mu_{1}^{\prime 2}-3 \mu_{1}^{\prime 4},
\end{aligned}
$$

$e$

$$
\mu_{r}^{\prime}=\frac{1}{g_{0}} \sum_{j=0}^{r}\left(\begin{array}{l}
r \\
j
\end{array}\right)\left(\sigma_{t}^{t-1}\right)^{j}\left(\mu_{t}^{t-1}\right)^{r-j} g_{j}
$$

em que

$$
g_{k}=\left(1+b_{0}\right) m_{k}^{\prime}+\sum_{i=1}^{8} b_{i} m_{k+i}^{\prime},
$$

e os $m_{j}^{\prime}$ são os momentos não centrais de uma distribuição normal de média $\mu_{d} e$ variância $\sigma_{d}^{2}, N\left(\mu_{d}, \sigma_{d}^{2}\right)$,

$$
\begin{aligned}
m_{j}^{\prime} & =\int_{-\infty}^{\infty} \frac{e^{-\frac{1}{2}\left(\frac{\zeta-\mu_{d}}{\sigma_{d}}\right)^{2}}}{\sqrt{2 \pi} \sigma_{d}} \zeta^{j} d \zeta, \\
\mu_{d} & =\frac{\rho_{t} \beta \sigma_{t}^{t-1}}{\beta v_{t}^{t-1}+\sigma_{\varepsilon}^{2}}, \\
\sigma_{d}^{2} & =\frac{\sigma_{\varepsilon}^{2}}{\beta^{2} v_{t}^{t-1}+\sigma_{\varepsilon}^{2}}, \\
\rho_{t} & =y_{t}-\mu_{\varepsilon}-\alpha-\beta \mu_{t}^{t-1} .
\end{aligned}
$$


Os momentos centrais $m_{j}^{\prime}$ são obtidos a partir da seguinte relação recursiva:

$$
\begin{aligned}
& m_{0}^{\prime}=1 \\
& m_{1}^{\prime}=\mu_{d} \\
& m_{j}^{\prime}=\mu_{d} m_{j-1}^{\prime}+(j-1) \sigma_{d}^{2} m_{j-2}^{\prime}, j \geq 2
\end{aligned}
$$

Os coeficientes $b_{i}$ em (6.46) são dados por:

$$
\begin{gathered}
b_{0}=\frac{\gamma_{2, t}^{t-1}}{8}+a_{0}\left(1+\frac{\gamma_{2, t}^{t-1}}{8}\right), \\
b_{1}=-\left(1+a_{0}\right) \frac{\gamma_{1, t}^{t-1}}{2}+a_{1}\left(1+\frac{\gamma_{2, t}^{t-1}}{8}\right), \\
b_{2}=-\left(1+a_{0}\right) \frac{\gamma_{2, t}^{t-1}}{4}-a_{1} \frac{\gamma_{1, t}^{t-1}}{2}+a_{2}\left(1+\frac{\gamma_{2, t}^{t-1}}{8}\right), \\
b_{3}=\left(1+a_{0}\right) \frac{\gamma_{1, t}^{t-1}}{6}-a_{1} \frac{\gamma_{2, t}^{t-1}}{4}-a_{2} \frac{\gamma_{1, t}^{t-1}}{2}+a_{3}\left(1+\frac{\gamma_{2, t}^{t-1}}{8}\right), \\
b_{4}=\left(1+a_{0}\right) \frac{\gamma_{2, t}^{t-1}}{24}+a_{1} \frac{\gamma_{1, t}^{t-1}}{6}-a_{2} \frac{\gamma_{2, t}^{t-1}}{4}-a_{3} \frac{\gamma_{1, t}^{t-1}}{2}+a_{4}\left(1+\frac{\gamma_{2, t}^{t-1}}{8}\right), \\
b_{5}=a_{1} \frac{\gamma_{2, t}^{t-1}}{24}+a_{2} \frac{\gamma_{1, t}^{t-1}}{6}-a_{3} \frac{\gamma_{2, t}^{t-1}}{4}-a_{4} \frac{\gamma_{1, t}^{t-1}}{2}, \\
b_{6}=a_{2} \frac{\gamma_{2, t}^{t-1}}{24}+a_{3} \frac{\gamma_{1, t}^{t-1}}{6}-a_{4} \frac{\gamma_{2, t}^{t-1}}{4}, \\
b_{7}=a_{3} \frac{\gamma_{2, t}^{t-1}}{24}+a_{4} \frac{\gamma_{1, t}^{t-1}}{6}, \\
b_{8}=a_{4} \frac{\gamma_{2, t}^{t-1}}{24} .
\end{gathered}
$$

Finalmente, os coeficiente $a_{i}$ nas expressões acima são dados por:

$$
\begin{gathered}
a_{0}=\frac{\gamma_{1, \varepsilon}}{6}\left[\left(\frac{\rho_{t}}{\sigma_{\varepsilon}}\right)^{3}-3\left(\frac{\rho_{t}}{\sigma_{\varepsilon}}\right)\right]+\frac{\gamma_{2, \varepsilon}}{24}\left[\left(\frac{\rho_{t}}{\sigma_{\varepsilon}}\right)^{4}-6\left(\frac{\rho_{t}}{\sigma_{\varepsilon}}\right)^{2}+3\right], \\
a_{1}=-\frac{\gamma_{1, \varepsilon}}{2} \frac{\beta \sigma_{\zeta}}{\sigma_{\varepsilon}}\left[\left(\frac{\rho_{t}}{\sigma_{\varepsilon}}\right)^{2}-1\right]-\frac{\gamma_{2, \varepsilon}}{6} \frac{\beta \sigma_{\zeta}}{\sigma_{\varepsilon}}\left[\left(\frac{\rho_{t}}{\sigma_{\varepsilon}}\right)^{3}-3\left(\frac{\rho_{t}}{\sigma_{\varepsilon}}\right)\right], \\
a_{2}=\frac{\gamma_{1, \varepsilon}}{2} \frac{\beta^{2} \sigma_{\zeta}^{2}}{\sigma_{\varepsilon}^{2}}\left(\frac{\rho_{t}}{\sigma_{\varepsilon}}\right)+\frac{\gamma_{2, \varepsilon}}{4} \frac{\beta^{2} \sigma_{\zeta}^{2}}{\sigma_{\varepsilon}^{2}}\left[\left(\frac{\rho_{t}}{\sigma_{\varepsilon}}\right)^{2}-1\right], \\
a_{3}=-\frac{\gamma_{1, \varepsilon}}{6} \frac{\beta^{3} \sigma_{\zeta}^{3}}{\sigma_{\varepsilon}^{3}}-\frac{\gamma_{2, \varepsilon}}{6} \frac{\beta^{3} \sigma_{\zeta}^{3}}{\sigma_{\varepsilon}^{3}}\left(\frac{\rho_{t}}{\sigma_{\varepsilon}}\right),
\end{gathered}
$$




$$
a_{4}=\frac{\gamma_{2, \varepsilon}}{24} \frac{\beta^{4} \sigma_{\zeta}^{4}}{\sigma_{\varepsilon}^{4}}
$$

Prova. A demonstração desta proposição encontra-se no apêndice $F$.

\subsubsection{Restrição do espaço paramétrico}

Nesta seção obtivemos um procedimento recursivo para cálculo da distribuição de probabilidades da variável latente, assumindo que esta densidade seja aproximada pela expansão de Gram-Charlier até a $4^{a}$ ordem. Entretanto, para que esta expansão de fato possa representar uma densidade é necessário impor restrições ao espaço paramétrico (veja Barton e Dennis (1952) e Jondeau e Rockinger (1999)). Em particular precisamos impor a condição que o polinômio $p_{4}(z)$ da expansão seja sempre positivo, $\forall z$, isto é:

$$
p_{4}(z)=1+\frac{\gamma_{1}}{6} H e_{3}(z)+\frac{\gamma_{2}}{24} H e_{4}(z) \geq 0,
$$

em que $H e_{i}(z)$ representa o polinômio de Hermite de ordem $i^{4}$.

Para obter o "envelope", isto é, a fronteira da região paramétrica, Jondeau e Rockinger (1999) encontram os valores de $\gamma_{1}$ e $\gamma_{2}$ que resolvem o seguinte sistema

$$
\begin{aligned}
p_{4}(z) & =1+\frac{\gamma_{1}}{6} H e_{3}(z)+\frac{\gamma_{2}}{24} H e_{4}(z)=0, \\
\frac{\partial p_{4}(z)}{\partial z} & =\frac{\gamma_{1}}{2} H e_{2}(z)+\frac{\gamma_{2}}{6} H e_{3}(z)=0 .
\end{aligned}
$$

A solução deste sistema, parametrizada em função de $z$, é dada por

$$
\begin{aligned}
& \gamma_{1}(z)=-24 \frac{H e_{3}(z)}{d(z)}=-24 \frac{\left(z^{3}-3 z\right)}{d(z)}, \\
& \gamma_{2}(z)=72 \frac{H e_{2}(z)}{d(z)}=72 \frac{\left(z^{2}-1\right)}{d(z)},
\end{aligned}
$$

${ }^{4} \mathrm{O}$ polinômio de Hermite de ordem $i$ é definido em função da $i$-ésima derivada da densidade da normal padrão $\varphi(z)$ :

Os seis primeiros polinômios são dados por

$$
\begin{aligned}
H e_{i}(z) & =\frac{(-1)^{i}}{\varphi(z)} \frac{\partial^{i} \varphi(z)}{\partial z^{i}} \\
\varphi(z) & =\frac{1}{\sqrt{2 \pi}} e^{-\frac{1}{2} z^{2}}
\end{aligned}
$$

$$
\begin{aligned}
& \operatorname{He}_{0}(z)=1 \\
& \operatorname{He}_{1}(z)=z \\
& \mathrm{He}_{2}(z)=z^{2}-1 \\
& \mathrm{He}_{3}(z)=z^{3}-3 z \\
& \mathrm{He}_{4}(z)=z^{4}-6 z^{2}+3 \\
& \mathrm{He}_{5}(z)=z^{5}-10 z^{3}+15 z \\
& \mathrm{He}_{6}(z)=z^{6}-15 z^{4}+45 z^{2}-15
\end{aligned}
$$


em que

$$
\begin{aligned}
d(z) & =4 H e_{3}^{2}(z)-3 H e_{2}(z) H e_{4}(z) \\
& =z^{6}-3 z^{4}+9 z^{2}+9 .
\end{aligned}
$$

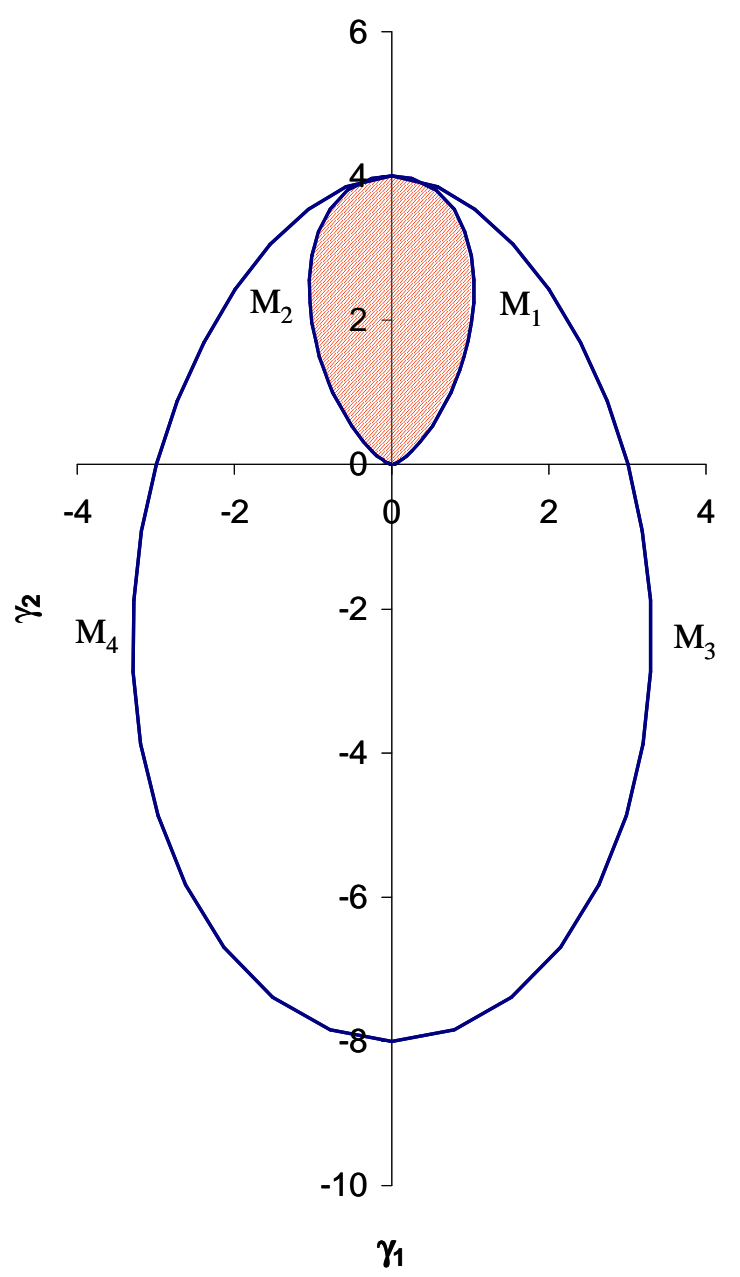

Figura 6.2: Espaço paramétrico $\left(\gamma_{1}, \gamma_{2}\right)$ para a expansão de Gram-Charlier. A linha cheia indica o "envelope" como indicado pelas equações (6.66) e (6.67), e a região hachurada representa o espaço paramétrico para que a expansão de Gram-Charlier constitua uma densidade de probabilidade.

A região hachurada da figura 6.2 indica o espaço paramétrico para $\left(\gamma_{1}, \gamma_{2}\right)$, que é dado para $|z| \geq \sqrt{3}$. O valor máximo para o coeficiente de assimetria $\gamma_{1}$ é alcançado no ponto $M_{1}=\left(\frac{\sqrt{6}}{\sqrt{3+\sqrt{6}}}, \sqrt{6}\right)=(1.0493,2.4508)$ e o mínimo no ponto $M_{2}=\left(-\frac{\sqrt{6}}{\sqrt{3+\sqrt{6}}}, \sqrt{6}\right)=$ $(-1.0493,2.4508)$. O coeficiente de excesso de curtose $\gamma_{2}$ máximo é alcançado no ponto $(0,4)$. 
Para implementar a restrição de positividade adotamos o seguinte procedimento ad hoc:

- Discretizamos $|z| \geq \sqrt{3}$, de maneira a aproximar o espaço paramétrico de $\left(\gamma_{1}, \gamma_{2}\right)$ por segmentos de reta.

- Caso $\gamma_{1} \in[-\sqrt{6} / \sqrt{3+\sqrt{6}}, \sqrt{6} / \sqrt{3+\sqrt{6}}]$ e $\gamma_{2} \notin[0,4]$, fixamos $\gamma_{1}$ e obtemos $\gamma_{2}^{\min }$ e $\gamma_{2}^{\max }$ por interpolação linear. Se $\gamma_{2} \notin\left[\gamma_{2}^{\min }, \gamma_{2}^{\max }\right]$, fixamos $\gamma_{2}$ no limite apropriado do intervalo.

- Caso $\gamma_{1} \notin[-\sqrt{6} / \sqrt{3+\sqrt{6}}, \sqrt{6} / \sqrt{3+\sqrt{6}}]$ e $\gamma_{2} \in[0,4]$, fixamos $\gamma_{2}$ e obtemos $\gamma_{1}^{\min }$ e $\gamma_{1}^{\max }$ por interpolação linear. Se $\gamma_{1} \notin\left[\gamma_{1}^{\min }, \gamma_{1}^{\max }\right]$, fixamos $\gamma_{1}$ no limite apropriado do intervalo.

- Caso $\gamma_{1} \notin[-\sqrt{6} / \sqrt{3+\sqrt{6}}, \sqrt{6} / \sqrt{3+\sqrt{6}}]$ e $\gamma_{2} \notin[0,4]$ fixamos arbitrariamente $\gamma_{1}$ e $\gamma_{2}$ conforme o quadrante

(a) se $\gamma_{1}>\sqrt{6} / \sqrt{3+\sqrt{6}}$ e $\gamma_{2}>4$, fixamos $\left(\gamma_{1}, \gamma_{2}\right)$ correspondentes à $z=-2$, i.e., $\left(\gamma_{1}, \gamma_{2}\right)=(48 / 61,216 / 61) \cong(0.79,3.54) ;$

(b) se $\gamma_{1}<-\sqrt{6} / \sqrt{3+\sqrt{6}}$ e $\gamma_{2}>4$, fixamos $\left(\gamma_{1}, \gamma_{2}\right)$ correspondentes à $z=2$, i.e., $\left(\gamma_{1}, \gamma_{2}\right)=(-48 / 61,216 / 61) \cong(-0.79,3.54)$

(c) se $\gamma_{1}<-\sqrt{6} / \sqrt{3+\sqrt{6}}$ e $\gamma_{2}<0$, fixamos $\left(\gamma_{1}, \gamma_{2}\right)$ correspondentes à $z=3$, i.e., $\left(\gamma_{1}, \gamma_{2}\right)=(-3 / 4,1)=(-0.75,1)$

(d) se $\gamma_{1}>\sqrt{6} / \sqrt{3+\sqrt{6}}$ e $\gamma_{2}<0$, fixamos $\left(\gamma_{1}, \gamma_{2}\right)$ correspondentes à $z=-3$, i.e., $\left(\gamma_{1}, \gamma_{2}\right)=(3 / 4,1)=(0.75,1)$.

Assim, em cada passo do algoritmo recursivo delineado na prop. (6.4) aplicamos o procedimento descrito acima, de maneira a manter $\left(\gamma_{1, t}^{t-1}, \gamma_{2, t}^{t-1}\right)$ e $\left(\gamma_{1, t}^{t}, \gamma_{2, t}^{t}\right)$ dentro do espaço paramétrico da "densidade" de Gram-Charlier até $4^{a}$ ordem.

\subsection{Sumário}

Neste capítulo vimos como obter estimativas para a variável latente $\psi_{t}$ do modelo SCD através de um método recursivo Bayesiano de integração numérica, através do filtro de Kalman (aproximação Gaussiana da distribuição dos erros) e através de um método recursivo baseado na expansão de Gram-Charlier até $4^{a}$ ordem (filtro de Gram-Charlier). Todos estes métodos assumem que o vetor de parâmetros $\theta$ seja conhecido. Mas o filtro de Kalman também permite a implementação de uma algoritmo de estimação (QML - quase verossimilhança) para estimação de $\theta$.

No próximo capítulo vamos analisar o desempenho dos diferentes métodos de estimação vistos no capítulo anterior e neste capítulo, através de simulação de processos de duração condicional estocástica. 


\section{Capítulo 7}

\section{Simulação}

Neste capítulo vamos verificar o desempenho dos métodos de estimação, conhecendo os verdadeiros parâmetros. Para isso vamos efetuar um conjunto de simulações de processos SCD, conhecido o vetor de parâmetros. Estas simulações podem ser facilmente efetuadas através do método da transformada inversa (veja, por exemplo, Robert e Casella (2004)), conhecida a função de distribuição da duração condicionada na variável latente, $x_{j} \mid \psi_{j}$, obtida na proposição 4.5.

Para visualizar o comportamento da variável duração e da log-duração (y), efetuamos um conjunto de simulações utilizando os parâmetros utilizados por Strickland et al. (2006): $\delta=0.033, \phi=0.95, \sigma_{\eta}=0.1$. A Figura 7.1 ilustra amostras das durações e respectivas log-durações com distribuições condicionais exponencial, Weibull e gama.

Podemos observar o comportamento de convergência da função característica empírica através das Figuras 7.2 e 7.3. As figuras ilustram o comportamento das partes real e imaginária da função característica empírica quando se aumenta o tamanho da amostra para 100, 1.000, 10.000 e 100.000, e a Figura 7.3 (baixo) ilustra a função característica calculada por (4.87). Podemos notar pelo exame das figuras, que mesmo em amostras reduzidas é possível distinguir o padrão da função característica, com concentração da informação em torno da origem. À medida que a amostra aumenta, as ondulações aleatórias se reduzem até praticamente desaparecerem para amostras de tamanho 100000. 

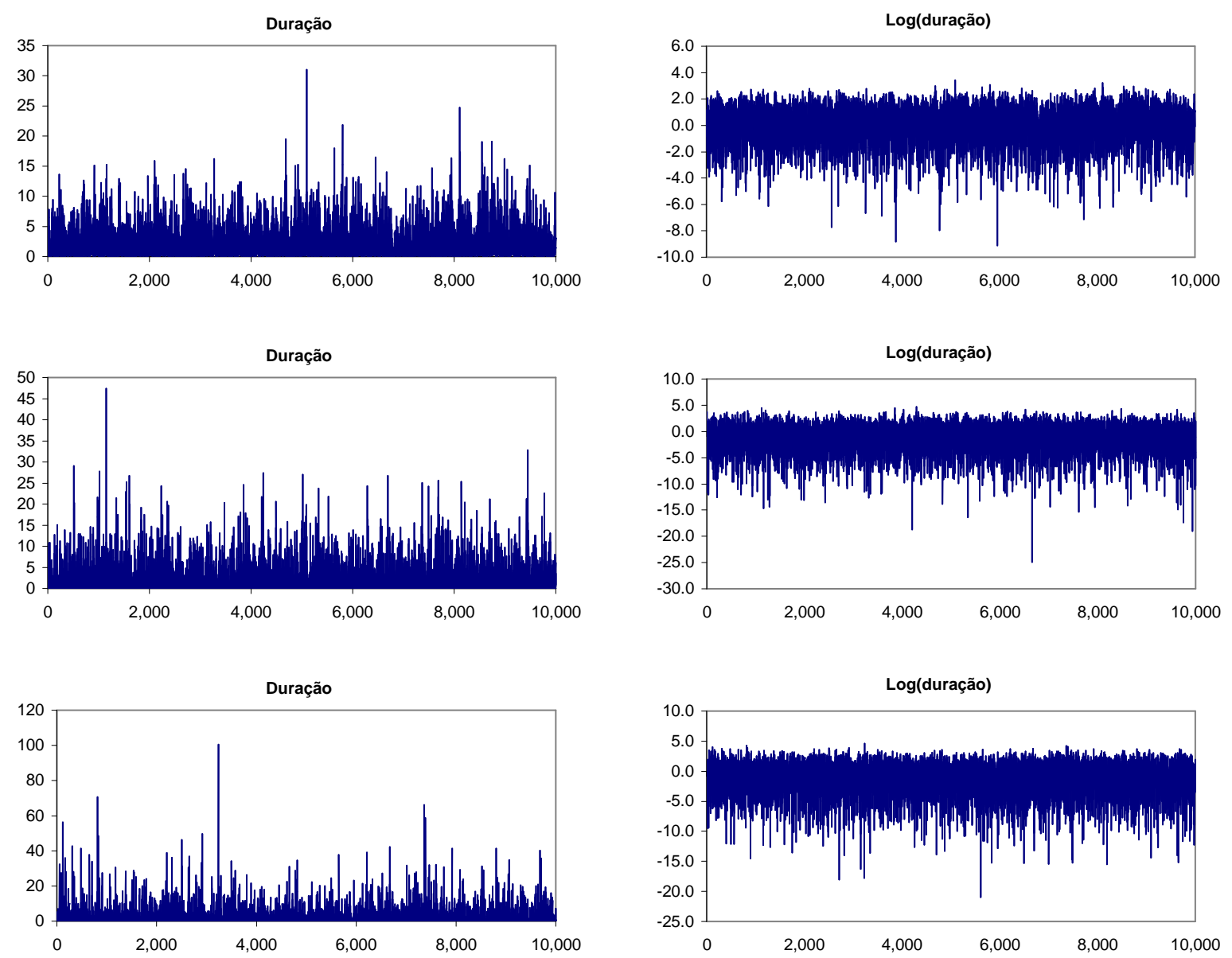

Figura 7.1: Amostras simuladas da duração (à esquerda) e log-duração (à direita) com distribuições condicionais exponencial (cima), Weibull $\lambda=0.5$ (meio) e gama $\lambda=0.5$ (baixo), e parâmetros $\delta=0.033, \phi=0.95, \sigma_{\eta}=0.1$. 

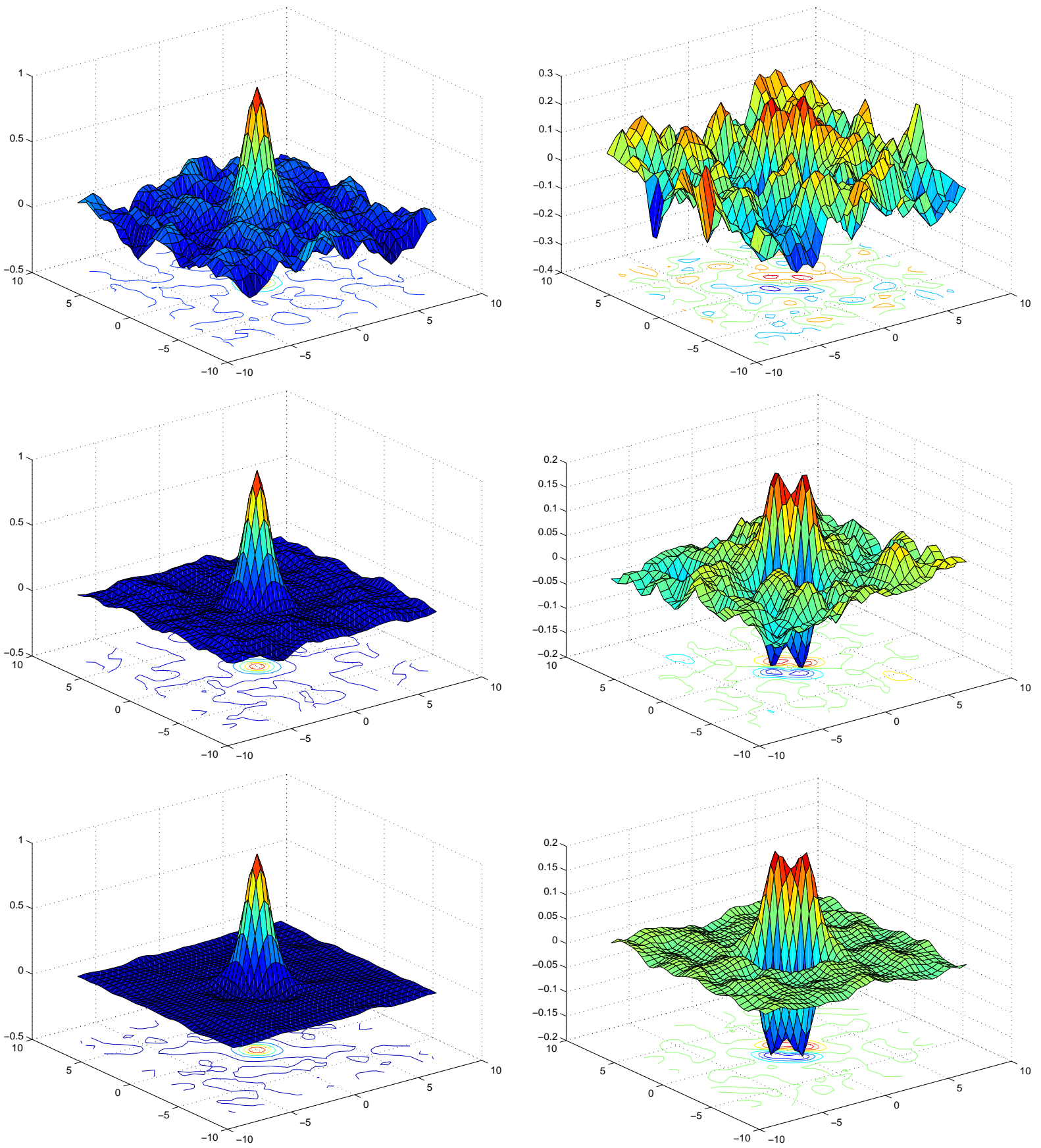

Figura 7.2: Partes reais (à esquerda) e imaginárias (à direita) da função característica empírica $(\mathrm{ECF}), \operatorname{Re} \varphi_{z_{n}}(r)$, para amostras de tamanho 100 (cima), 1000 (meio) e 10000 (baixo), em função de $r=\left(r_{1}, r_{2}\right)$, com parâmetros $\delta=0.033, \phi=0.95, \sigma_{\eta}=0.1, \lambda=1$ (distribuição exponencial), $p=1$, e usando para $r_{\zeta}$ os nós da quadratura de Gauss-Hermite de 39 pontos. 

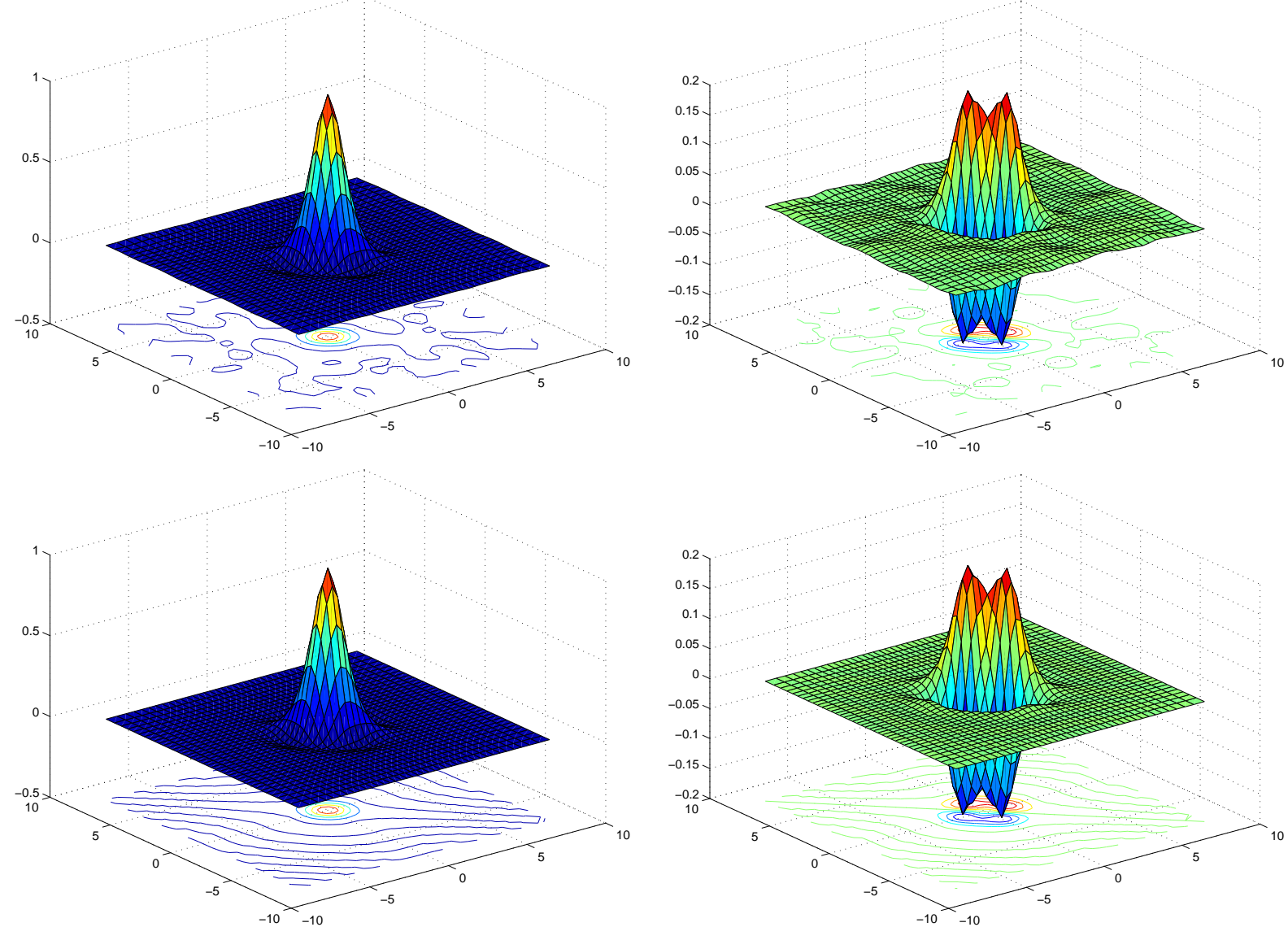

Figura 7.3: Partes reais (à esquerda) e imaginárias (à direita) da função característica empírica $(\mathrm{ECF}), \operatorname{Re} \varphi_{z_{n}}(r)$, para amostra de tamanho 100.000 (cima) e para a função característica $(\mathrm{CF}), \operatorname{Re} \varphi_{z}(r)$, (baixo) em função de $r=\left(r_{1}, r_{2}\right)$, com parâmetros $\delta=0.033, \phi=0.95$, $\sigma_{\eta}=0.1, \lambda=1$ (distribuição exponencial), $p=1$, e usando para $r_{\zeta}$ os nós da quadratura de Gauss-Hermite de 39 pontos. 


\subsection{Número de nós para quadratura de Gauss-Hermite}

As integrações necessárias para o método CECF foram efetuadas através do método de quadratura de Gauss-Hermite, que depende de um parâmetro: o número de "nós" para a integração. Na figura 7.4 plotamos o número de nós a uma distância da origem (3 e 10), em função do número de nós da quadratura. Note que o algoritmo utilizado para a geração dos nós ${ }^{1}$ fica instável a partir de 198 nós. Assim, o número máximo de nós que poderemos usar nas integrações é 197.
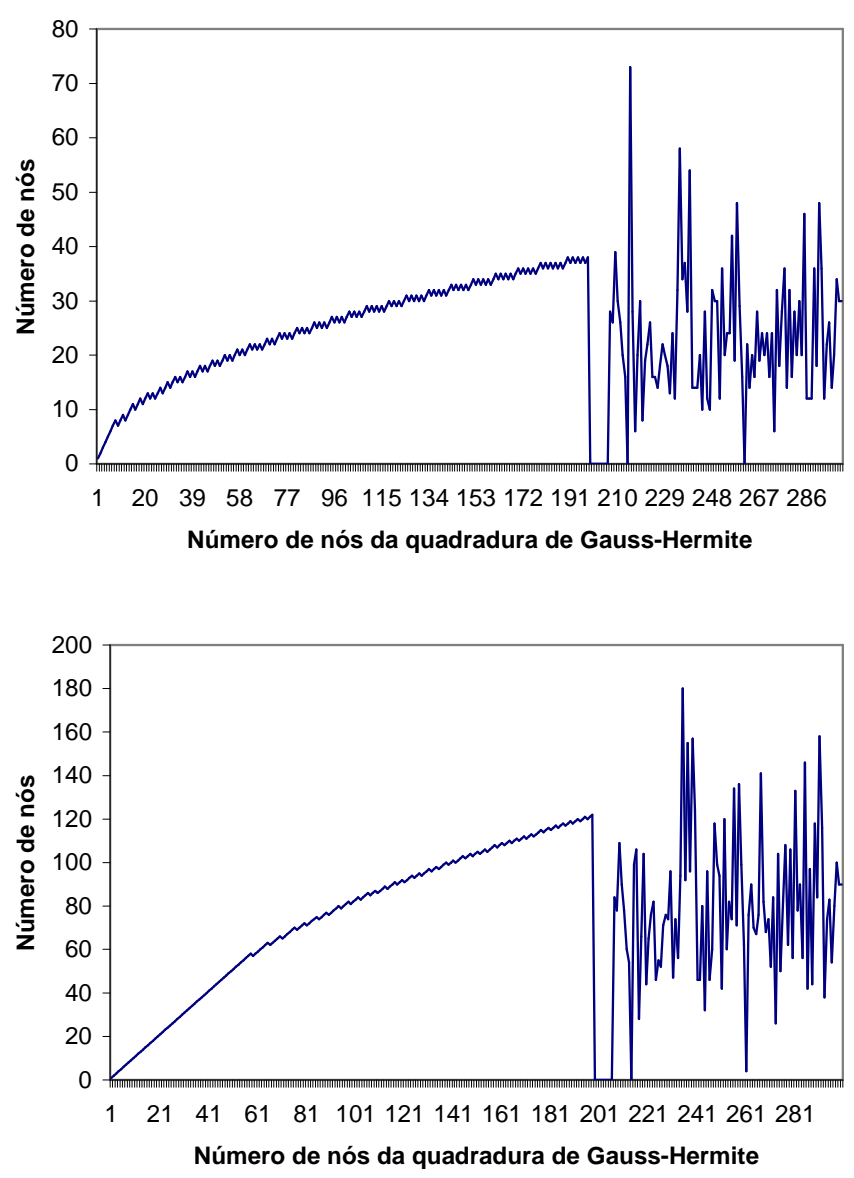

Figura 7.4: Número de nós a uma distância da origem (abscissas) em função do número de nós da quadratura de Gauss-Hermite (ordenadas): na figura de cima a distância máxima é 3 , e na de baixo a distância máxima é 10 .

Para procurar um valor inferior para o número de nós, efetuamos a integral

$$
\int_{\mathbb{R}^{p+1}}|\varphi(r)| e^{-r^{\prime} r} d r
$$

1 Press et al. (1997), algoritmo gauher, pg. 154. 
para o caso da distribuição de Weibull de parâmetros $\theta=(0.033,0.95,0.1,0.5)^{\prime}$. Evidentemente a integral acima não é a função objetivo que precisamos para obter os estimadores CECF; mesmo assim, nós a utilizamos para ter uma "idéia" do número de nós necessários para obter uma certa "qualidade" na integração. A figura 7.5 ilustra o resultado da integral acima em função do número de nós, mas limitada aos nós a uma distância de no máximo 3 da origem. Pode-se notar que a integral se estabiliza a partir de cerca de 50 nós de quadratura.

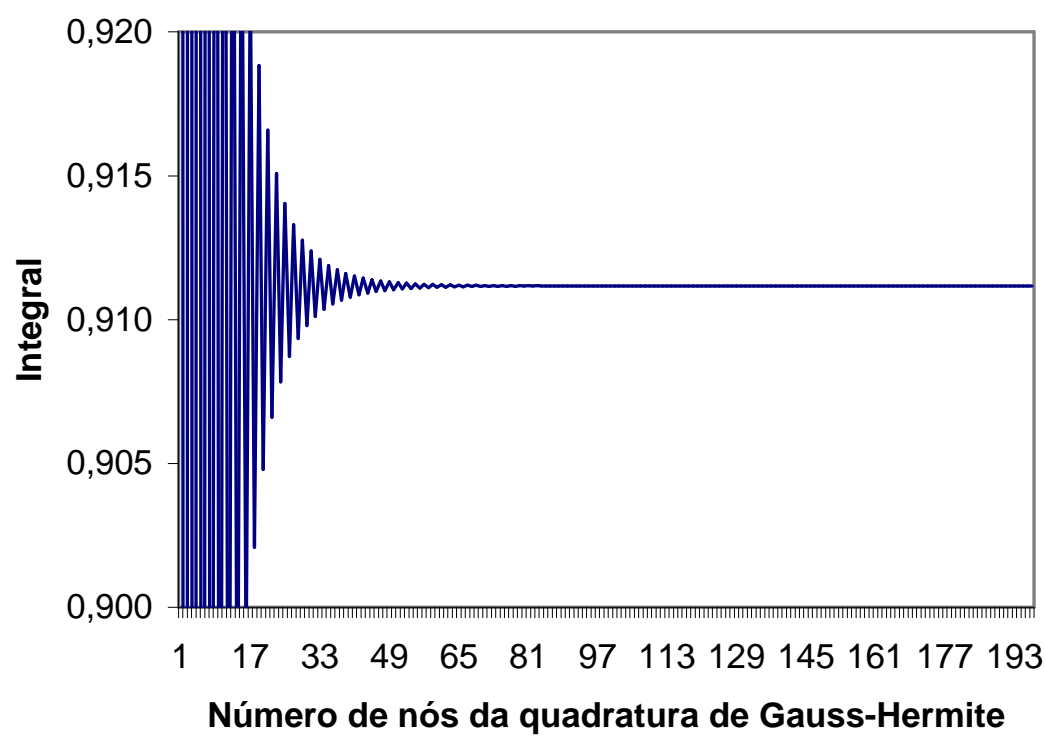

Figura 7.5: Resultado da integral $\int|\varphi(r)| e^{-r^{\prime} r} d r$, em função do número de nós da quadratura de Gauss-Hermite. 
Tabela 7.1: Resumo dos resultados (média, desvio padrão, erro quadrático médio e desvio absoluto médio) das estimativas amostrais dos parâmetros de 1000 simulações usando os algoritmos QML, CECF e DECF, para distribuições exponencial, Weibull e gama com parâmetros $\delta=0.033, \phi=0.95, \sigma_{\eta}=0.1, \lambda$ variável conforme tabela, tamanho de amostra 10000 e ponto inicial $\theta=(0,0.5,0.5,1)^{\prime}$.

$\mathrm{O}$ algoritmo de otimização utilizado foi o fminsearch do Matlab para os métodos QML e CECF e o lsqnonlin para o método DECF, com parâmetros tol $X=10^{-6}$ (tolerância da variável de decisão) e tolFun $=10^{-6}$ (tolerância da função objetivo). Para os algoritmos CECF e DECF foram usados 130 nós da quadratura de Gauss-Hermite, limitados a uma distância 3 da origem. $\mathrm{EQM}=$ erro quadrático médio e DAM = desvio absoluto médio.

\begin{tabular}{|c|c|c|c|c|c|c|c|c|c|c|c|c|c|c|}
\hline & \multicolumn{4}{|c|}{ QML } & \multicolumn{4}{|c|}{ CECF } & \multicolumn{4}{|c|}{ DECF } \\
\hline & & & $\delta$ & $\phi$ & $\sigma_{\eta}$ & $\lambda$ & $\delta$ & $\phi$ & $\sigma_{\eta}$ & $\lambda$ & $\delta$ & $\phi$ & $\sigma_{\eta}$ & $\lambda$ \\
\hline \multirow{4}{*}{\multicolumn{2}{|c|}{ Exponencial $\lambda=1$}} & Média & 0.034 & 0.948 & 0.101 & 1.000 & 0.041 & 0.938 & 0.103 & 1.000 & 0.035 & 0.948 & 0.100 & 1.000 \\
\hline & & Desvio padrão & 0.008 & 0.012 & 0.015 & 0.000 & 0.045 & 0.069 & 0.043 & 0.000 & 0.011 & 0.016 & 0.016 & 0.000 \\
\hline & & EQM & 0.000 & 0.000 & 0.000 & 0.000 & 0.002 & 0.005 & 0.002 & 0.000 & 0.000 & 0.000 & 0.000 & 0.000 \\
\hline & & DAM & 0.006 & 0.009 & 0.011 & 0.000 & 0.016 & 0.024 & 0.022 & 0.000 & 0.007 & 0.010 & 0.012 & 0.000 \\
\hline \multirow[t]{4}{*}{ Weibull } & $\lambda=2$ & Média & 0.033 & 0.949 & 0.100 & 2.000 & 0.033 & 0.949 & 0.100 & 2.000 & 0.033 & 0.949 & 0.100 & 2.000 \\
\hline & & Desvio padrão & 0.004 & 0.006 & 0.006 & 0.022 & 0.004 & 0.006 & 0.006 & 0.022 & 0.004 & 0.006 & 0.006 & 0.023 \\
\hline & & EQM & 0.000 & 0.000 & 0.000 & 0.001 & 0.000 & 0.000 & 0.000 & 0.000 & 0.000 & 0.000 & 0.000 & 0.000 \\
\hline & & DAM & 0.003 & 0.005 & 0.005 & 0.018 & 0.003 & 0.005 & 0.005 & 0.018 & 0.003 & 0.005 & 0.005 & 0.019 \\
\hline \multirow[t]{4}{*}{ Gama } & $\lambda=2$ & Média & 0.034 & 0.949 & 0.101 & 2.001 & 0.034 & 0.949 & 0.101 & 2.001 & 0.034 & 0.949 & 0.101 & 2.001 \\
\hline & & Desvio padrão & 0.005 & 0.007 & 0.008 & 0.031 & 0.005 & 0.007 & 0.008 & 0.034 & 0.005 & 0.007 & 0.009 & 0.041 \\
\hline & & EQM & 0.000 & 0.000 & 0.000 & 0.001 & 0.000 & 0.000 & 0.000 & 0.001 & 0.000 & 0.000 & 0.000 & 0.001 \\
\hline & & DAM & 0.004 & 0.006 & 0.007 & 1.501 & 0.004 & 0.006 & 0.007 & 1.501 & 0.004 & 0.006 & 0.007 & 1.501 \\
\hline \multirow[t]{4}{*}{ Weibull } & $\lambda=0.5$ & Média & 0.157 & 0.749 & 0.202 & 0.503 & 0.141 & 0.782 & 0.142 & 0.501 & 0.144 & 0.780 & 0.151 & 0.501 \\
\hline & & Desvio padrão & 0.228 & 0.352 & 0.188 & 0.018 & 0.250 & 0.376 & 0.140 & 0.007 & 0.202 & 0.307 & 0.123 & 0.008 \\
\hline & & EQM & 0.067 & 0.164 & 0.046 & 0.000 & 0.074 & 0.170 & 0.021 & 0.000 & 0.053 & 0.123 & 0.018 & 0.000 \\
\hline & & DAM & 0.131 & 0.209 & 0.112 & 0.006 & 0.120 & 0.186 & 0.086 & 0.006 & 0.116 & 0.178 & 0.084 & 0.006 \\
\hline \multirow[t]{4}{*}{ Gama } & $\lambda=0.5$ & Média & 0.087 & 0.866 & 0.140 & 0.502 & 0.062 & 0.906 & 0.114 & 0.501 & 0.063 & 0.904 & 0.121 & 0.501 \\
\hline & & Desvio padrão & 0.164 & 0.252 & 0.117 & 0.020 & 0.101 & 0.157 & 0.081 & 0.008 & 0.084 & 0.129 & 0.061 & 0.009 \\
\hline & & EQM & 0.030 & 0.071 & 0.015 & 0.248 & 0.011 & 0.027 & 0.007 & 0.249 & 0.008 & 0.019 & 0.004 & 0.249 \\
\hline & & DAM & 0.060 & 0.094 & 0.055 & 0.007 & 0.043 & 0.067 & 0.048 & 0.006 & 0.037 & 0.056 & 0.038 & 0.007 \\
\hline
\end{tabular}

\subsection{Resultados da simulação}

\subsubsection{Desempenho dos estimadores}

Com o objetivo de avaliar o desempenho dos diversos métodos de estimação efetuamos 1000 simulações de tamanho 10 000, para durações com distribuições condicionais exponencial, Weibull e gama, de parâmetros $\delta=0.033, \phi=0.95, \sigma_{\eta}=0.1$ e $\lambda=2$ e 0.5 . Para cada simulação utilizamos os algoritmos QML, CECF e DECF, apresentando um resumo dos resultados na Tabela 7.1. Note que para $\lambda=2$ os três métodos fornecem resultados parecidos, tanto em erro quadrático médio (EQM) quanto em desvio absoluto médio (DAM), mas para $\lambda=0.5$ os métodos CECF e DECF apresentam menor EQM e DAM que o método QML, com o DECF com desempenho ligeiramente superior ao CECF.

Para melhor visualizar o comportamento dos estimadores apresentamos, também, os his- 
Tabela 7.2: Estimativa dos desvios padrão do estimador CECF para o modelo SCD de parâmetros $\delta=0.033, \phi=0.95, \sigma_{\eta}=0.1$, com $\lambda$ e distribuições variáveis conforme a tabela, para amostras de tamanho 10 000. Mais detalhes sobre as estimativas podem ser encontrados no texto e no apêndice G.

\begin{tabular}{llllllr}
\hline & & \multicolumn{4}{c}{ Desvio padrão } & Tempo \\
\cline { 3 - 6 } & & $\delta$ & $\phi$ & $\sigma_{\eta}$ & $\lambda$ & $(h)$ \\
\hline Exponencial & $\lambda=1$ & 0.109 & 0.166 & 0.167 & & 35.5 \\
Weibull & $\lambda=2$ & 0.074 & 0.112 & 0.115 & 0.039 & 43.1 \\
Gama & $\lambda=2$ & 0.095 & 0.145 & 0.148 & 0.049 & 47.5 \\
Weibull & $\lambda=0.5$ & 0.650 & 0.986 & 1.003 & 0.007 & 39.2 \\
Gama & $\lambda=0.5$ & 0.264 & 0.400 & 0.403 & 0.007 & 43.1 \\
\hline
\end{tabular}

Tabela 7.3: Estimativa dos desvios padrão do estimador DECF para o modelo SCD de parâmetros $\delta=0.033, \phi=0.95, \sigma_{\eta}=0.1$, com $\lambda$ e distribuições variáveis conforme a tabela, para amostras de tamanho 10 000. Mais detalhes sobre as estimativas podem ser encontrados no texto e no apêndice G.

\begin{tabular}{llllllr}
\hline & & \multicolumn{4}{c}{ Desvio padrão } & Tempo \\
\cline { 3 - 6 } & & $\delta$ & $\phi$ & $\sigma_{\eta}$ & $\lambda$ & $(h)$ \\
\hline Exponencial & $\lambda=1$ & 0.127 & 0.193 & 0.192 & & 32.9 \\
Weibull & $\lambda=2$ & 0.118 & 0.179 & 0.185 & 0.090 & 43.1 \\
Gama & $\lambda=2$ & 0.179 & 0.272 & 0.278 & 0.128 & 39.3 \\
Weibull & $\lambda=0.5$ & 1.135 & 1.722 & 1.741 & 0.010 & 40.1 \\
Gama & $\lambda=0.5$ & 0.324 & 0.491 & 0.494 & 0.010 & 38.0 \\
\hline
\end{tabular}

togramas das estimativas amostrais dos parâmetros obtidos com a distribuição Weibull de parâmetros $\theta=\left(\delta, \phi, \sigma_{\eta}, \lambda\right)^{\prime}=(0.033,0.95,0.1,0.5)^{\prime}$ nas figuras 7.6 a 7.9, Para fins de comparação com os resultados simulados ilustramos nas tabelas 7.2 e 7.3 as estimativas dos desvios padrão dos estimadores dos métodos CECF e DECF, calculados de acordo com os resultados do capítulo 5, Estes desvios padrão foram calculados como a raiz quadrada da diagonal da matriz $V / n$, onde a matriz $V=B\left(\theta_{0}\right)^{-1} A\left(\theta_{0}\right) B\left(\theta_{0}\right)^{-1}$. As matrizes $A\left(\theta_{0}\right)$ foram estimadas por (5.10) para CECF e (5.41) para DECF, e as matrizes $B\left(\theta_{0}\right)$ foram estimadas por (5.18) para CECF e (5.40) para DECF. Para todas as integrações usamos 50 nós da quadratura de Gauss-Hermite e para cálculo da matriz $\Omega_{n}$ utilizamos truncamos a série em $m=100 \mathrm{em}$ (5.11). Note, em particular, os elevados tempos de processamento (cerca de 2 dias), apesar do truncamento aparentemente "curto" da série, principalmente se observarmos o comportamento de $\Omega_{n}$ obtido na figura 5.1 .

Nas figuras 7.6 a 7.9 ilustramos o histograma das 1000 simulações do processo SCD com distribuição Weibull e $\lambda=0.5$. Note como os estimadores CECF e DECF "suavizaram" a bimodalidade apresentada pelo estimador QML. Note também que, apesar do grande tamanho da amostra (10 000 observações), as distribuições dos estimadores é acentuadamente assimétrica e com excesso de curtose, sugerindo que a velocidade de convergência dos estimadores 
para a distribuição normal é possivelmente bastante lenta.

\subsubsection{Desempenho dos filtros}

Para avaliar o comportamento dos diversos filtros testamos os métodos para as distribuições exponencial, Weibull e gama, com parâmetros $\theta=(0.033,0.95,0.10, \lambda)$ e $\lambda=0.5$ e 2 . Os resultados encontram-se nas figuras 7.10 a 7.14, respectivamente.

Na Tabela 7.4 apresentamos o erro quadrático médio amostral (EQM), o desvio absoluto médio (DAM) e a mediana dos desvios absolutos (MDA) para a variável latente $\psi_{t}$, obtidos com cada um dos filtros propostos no capítulo [6, i.e., filtros de Kalman, Bayesiano e de Gram-Charlier. Nota-se que o método de integração numérica (Bayesiano) apresentou menor EQM, DAM e MDA para praticamente todas as distribuições simuladas. 


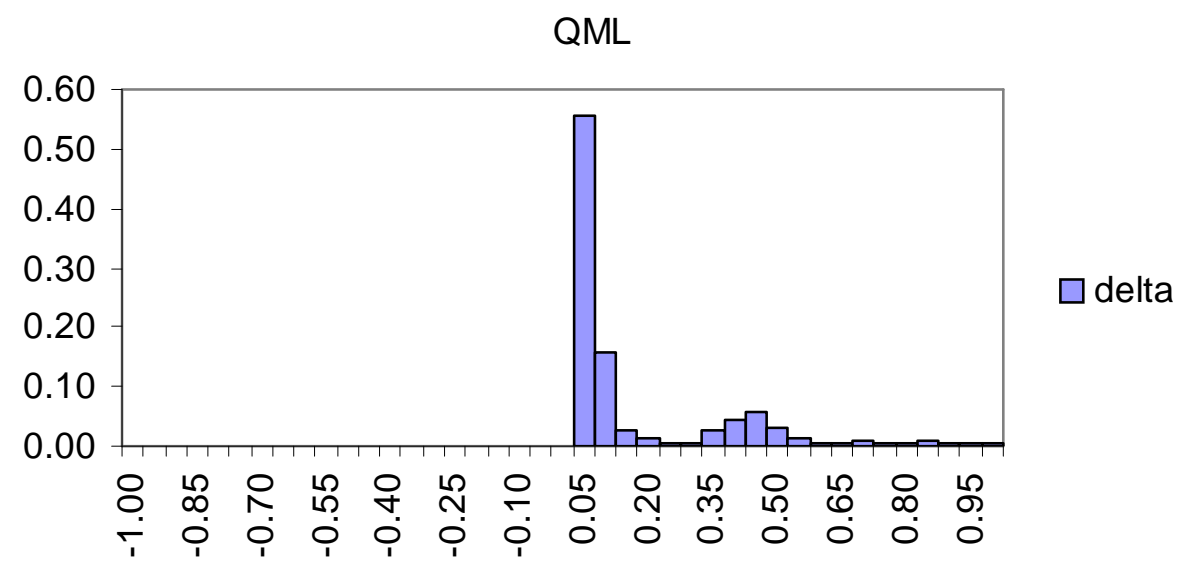

CECF

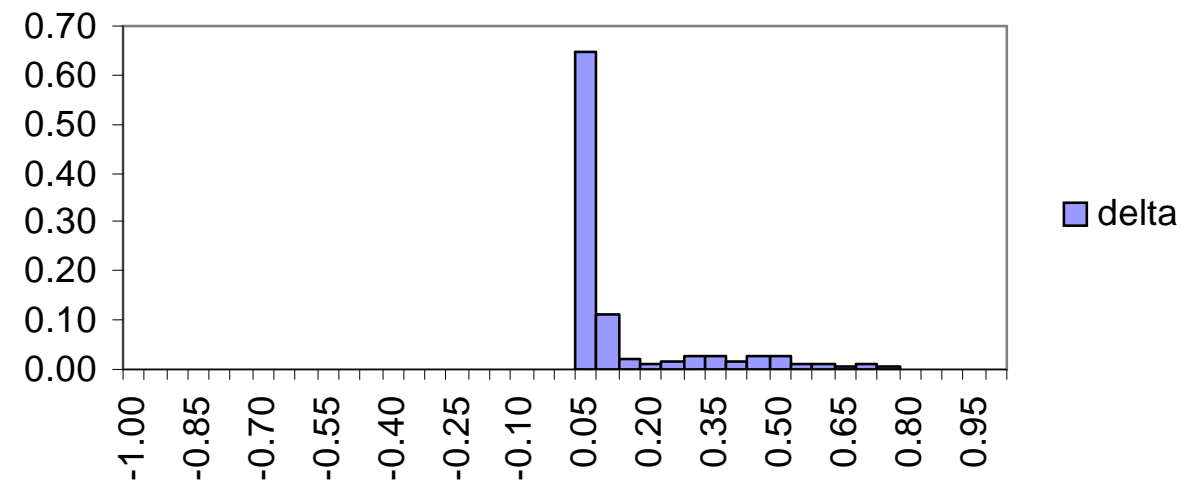

DECF

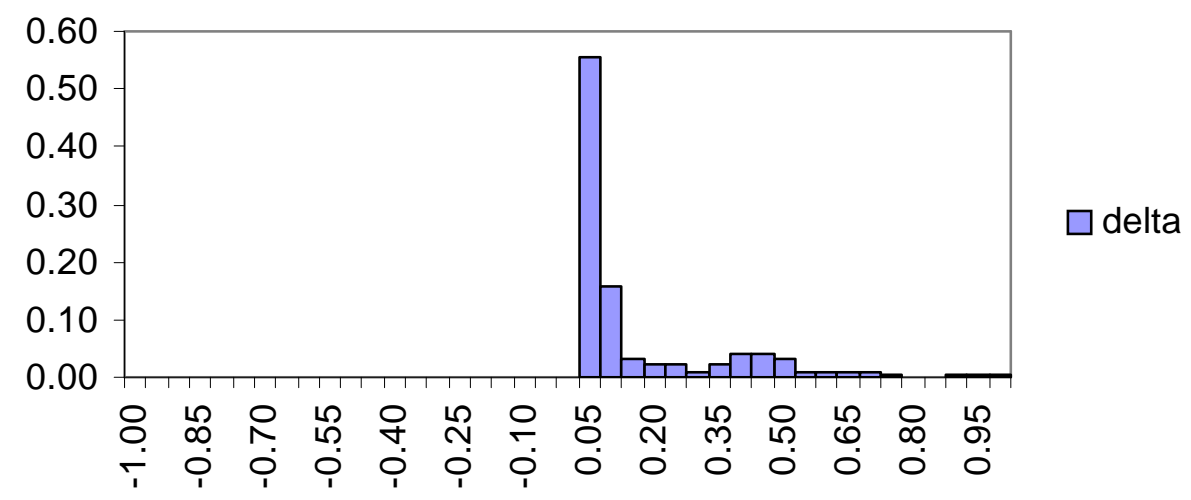

Figura 7.6: Histograma das estimativas do parâmetro $\delta$ obtidas com os métodos QML, CECF e QML em 1000 simulações para a distribuição Weibull de parâmetros $\theta=\left(\delta, \phi, \sigma_{\eta}, \lambda\right)^{\prime}=$ $(0.033,0.95,0.1,0.5)^{\prime}$. 

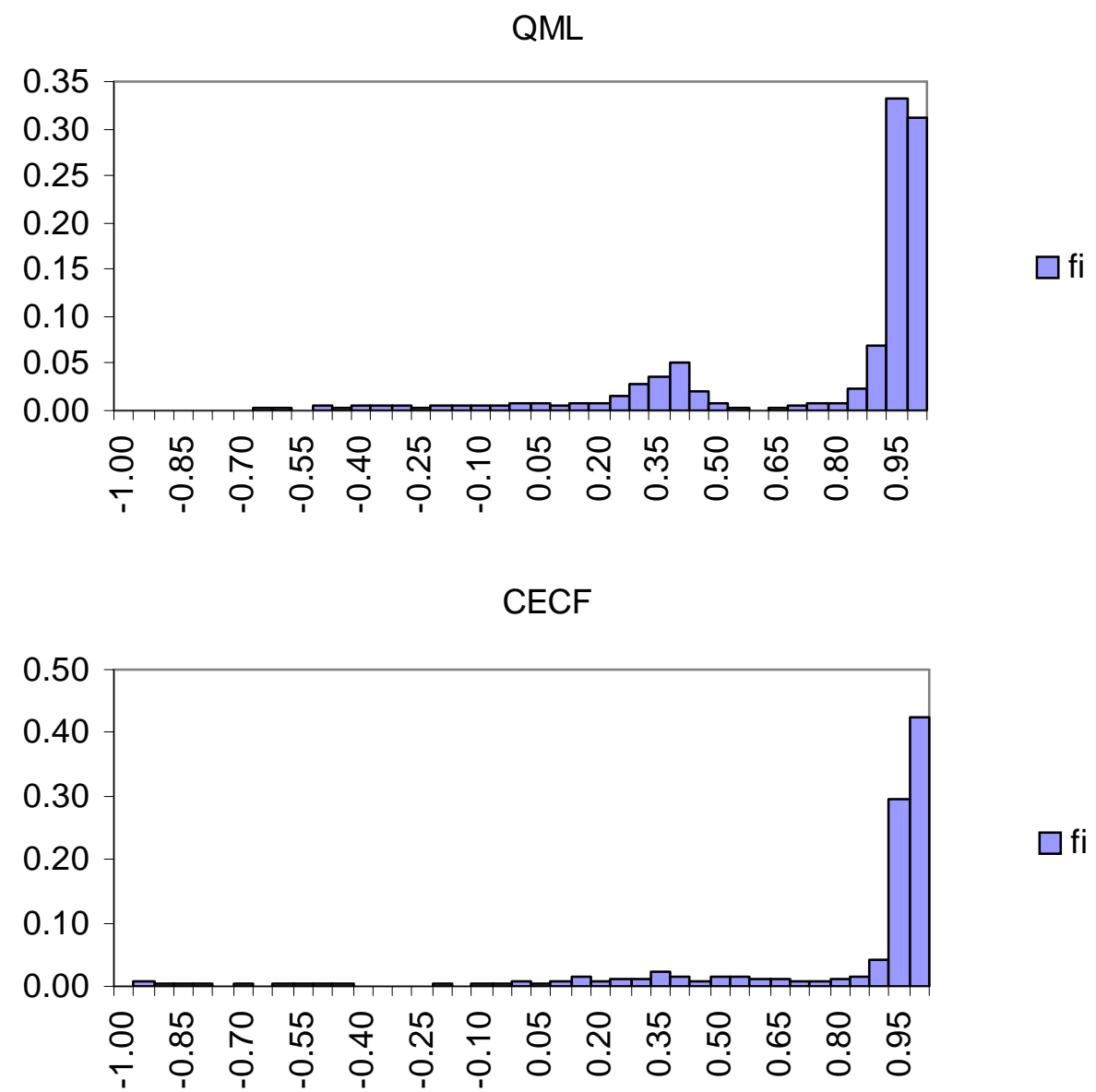

DECF

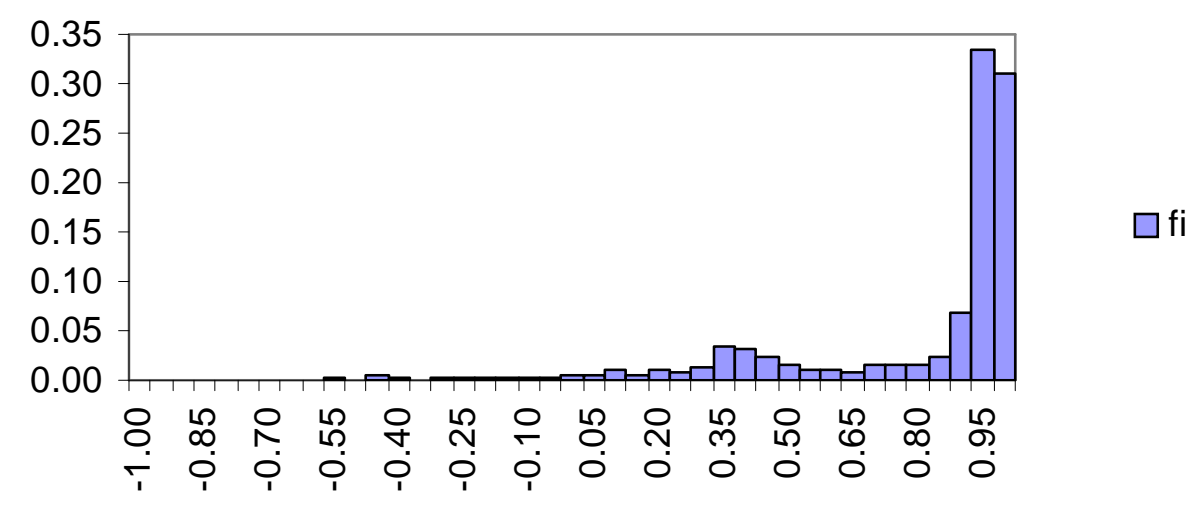

Figura 7.7: Histograma das estimativas do parâmetro $\phi$ obtidas com os métodos QML, CECF e QML em 1000 simulações para a distribuição Weibull de parâmetros $\theta=$ $(0.033,0.95,0.1,0.5)^{\prime}$. 
QML

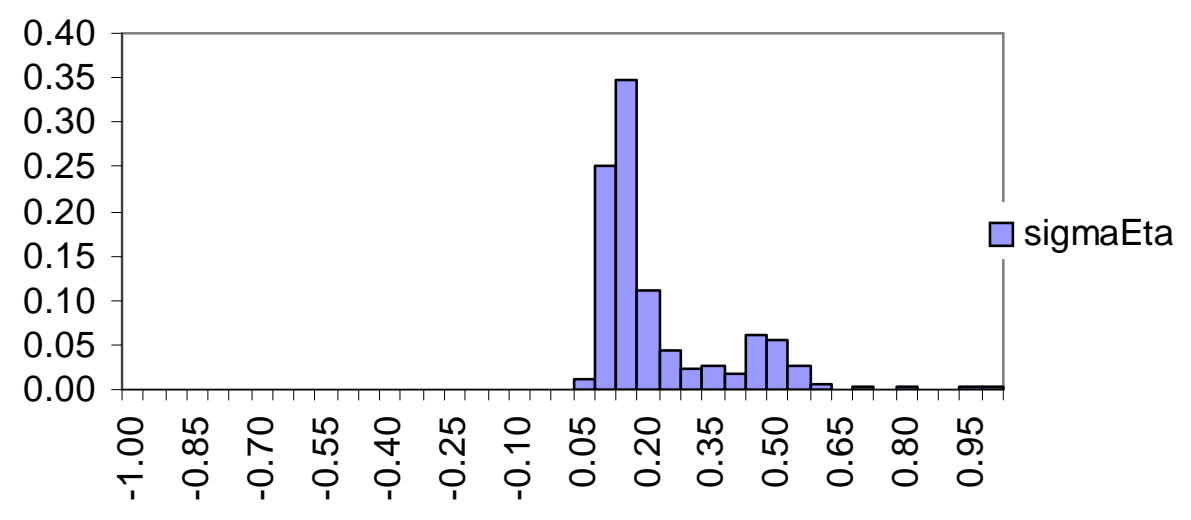

CECF

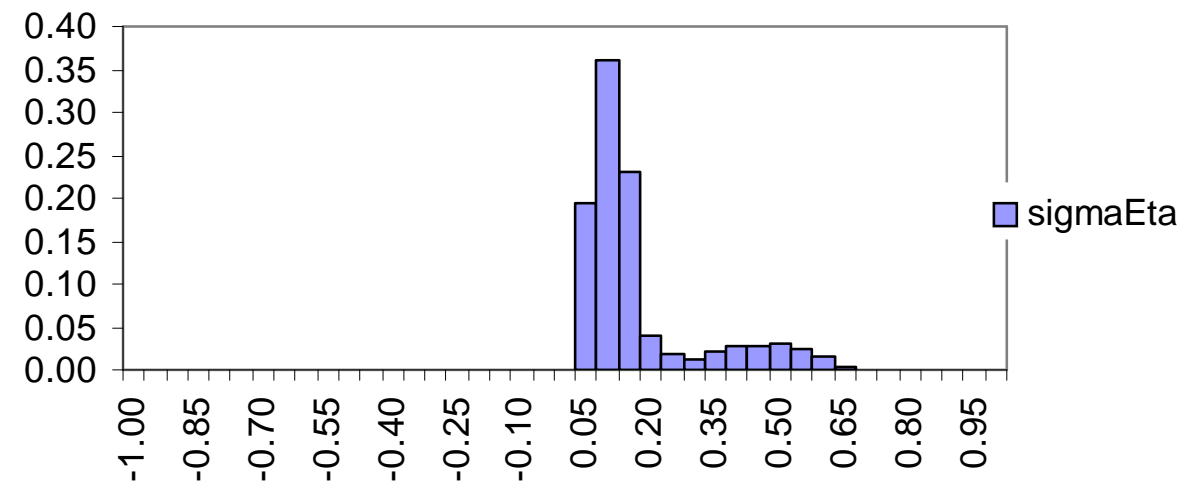

DECF

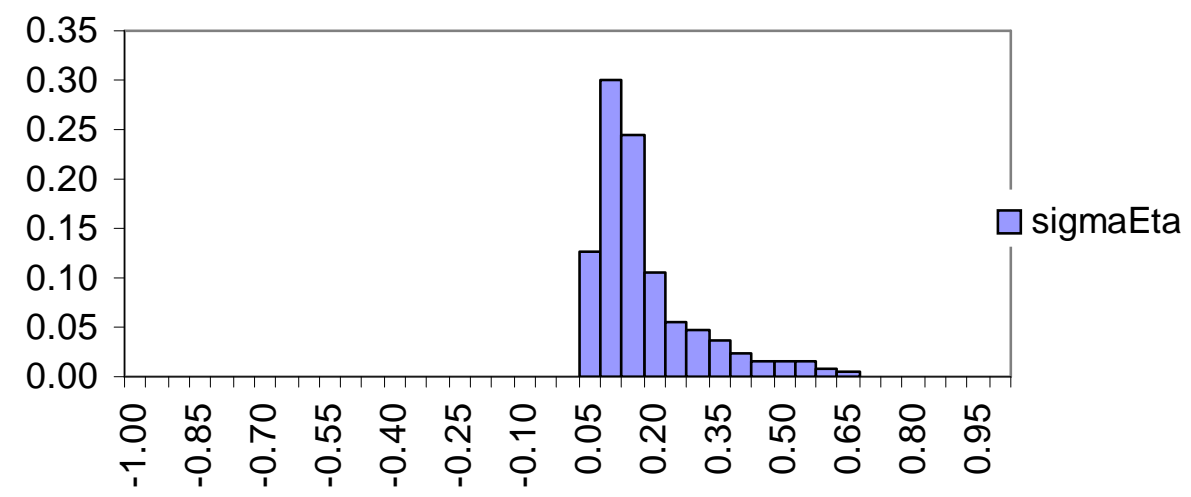

Figura 7.8: Histograma das estimativas do parâmetro $\sigma_{\eta}$ obtidas com os métodos QML, CECF e QML em 1000 simulações para a distribuição Weibull de parâmetros $\theta=$ $(0.033,0.95,0.1,0.5)^{\prime}$. 

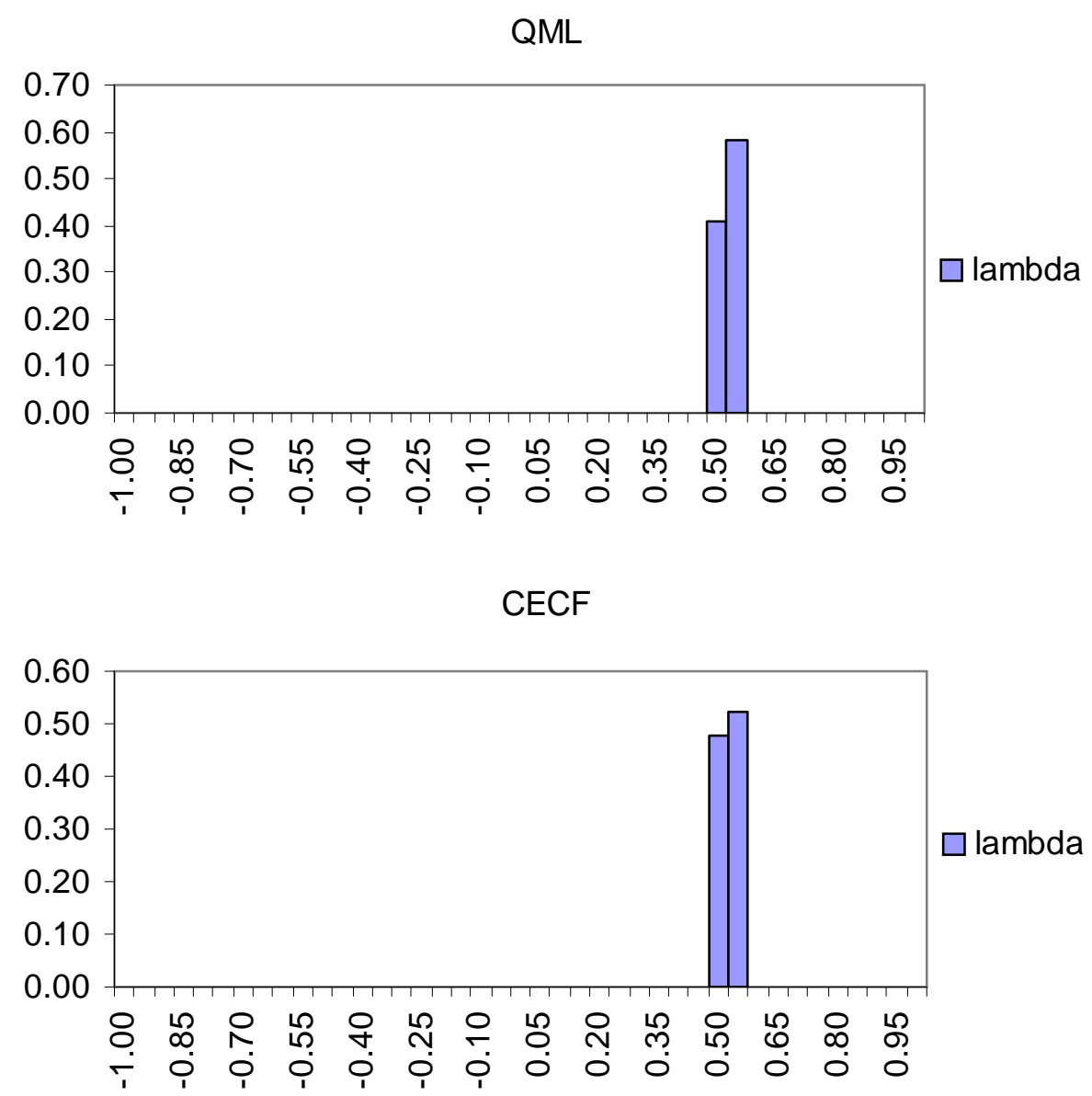

DECF

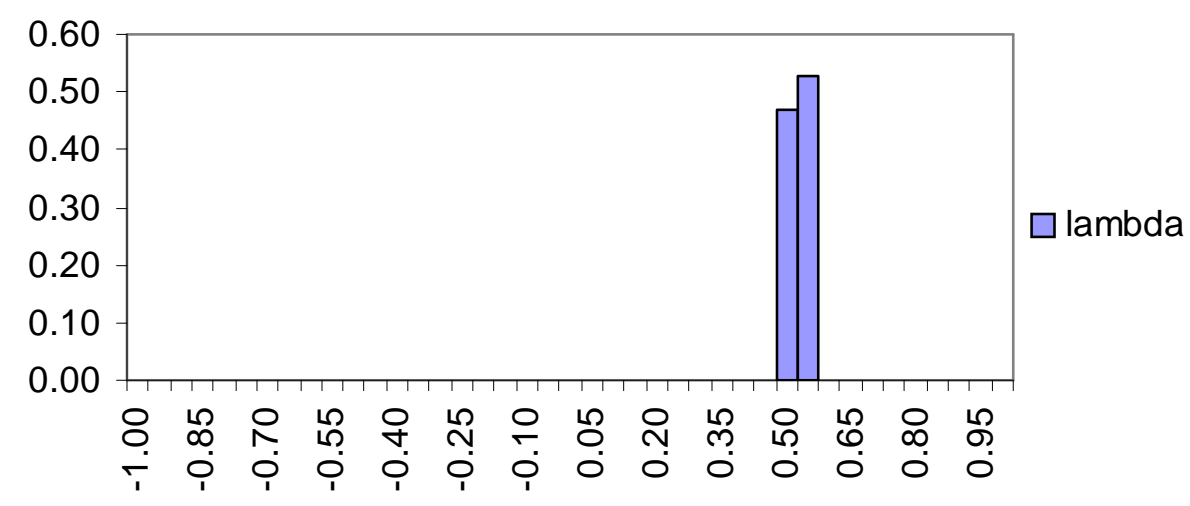

Figura 7.9: Histograma das estimativas do parâmetro $\lambda$ obtidas com os métodos QML, CECF e QML em 1000 simulações para a distribuição Weibull de parâmetros $\theta=$ $(0.033,0.95,0.1,0.5)^{\prime}$. 
Tabela 7.4: Erro quadrático médio (EQM), desvio absoluto médio (DAM) e mediana dos desvios absolutos (MDA) dos filtros de Kalman, Bayesiano e de Gram-Charlier utilizados para estimação da variável latente, obtidos com simulações de amostras de tamanho 1000 , calculados nos parâmetros $\theta=(0.033,0.95,0.10, \lambda)$ e $\lambda=0.5,1$ (dist. exponencial) e 2 .

\begin{tabular}{lrrr}
\hline EQM & Kalman & Bayesian & Gram-Charlier \\
\hline Exponencial & 0.069 & 0.060 & 0.107 \\
Weibull $\lambda=2$ & 0.047 & 0.037 & 0.057 \\
Gama $\lambda=2$ & 0.057 & 0.058 & 0.091 \\
Weibull $\lambda=0.5$ & 0.092 & 0.087 & 0.165 \\
Gama $\lambda=0.5$ & 0.052 & 0.049 & 0.092 \\
\hline
\end{tabular}

\begin{tabular}{|c|c|c|c|}
\hline$\overline{\mathrm{DAM}}$ & Kalman & Bayesian & Gram-Charlier \\
\hline Exponencial & 0.211 & 0.195 & 0.261 \\
\hline Weibull $\lambda=2$ & 0.175 & 0.156 & 0.194 \\
\hline Gama $\lambda=2$ & 0.187 & 0.188 & 0.241 \\
\hline Weibull $\lambda=0.5$ & 0.240 & 0.233 & 0.336 \\
\hline Gama $\lambda=0.5$ & 0.185 & 0.182 & 0.244 \\
\hline$\overline{\mathrm{MDA}}$ & Kalman & Bayesian & Gram-Charlier \\
\hline Exponencial & 0.177 & 0.160 & 0.208 \\
\hline Weibull $\lambda=2$ & 0.150 & 0.133 & 0.172 \\
\hline Gama $\lambda=2$ & 0.150 & 0.151 & 0.207 \\
\hline Weibull $\lambda=0.5$ & 0.194 & 0.186 & 0.300 \\
\hline Gama $\lambda=0.5$ & 0.156 & 0.164 & 0.208 \\
\hline
\end{tabular}



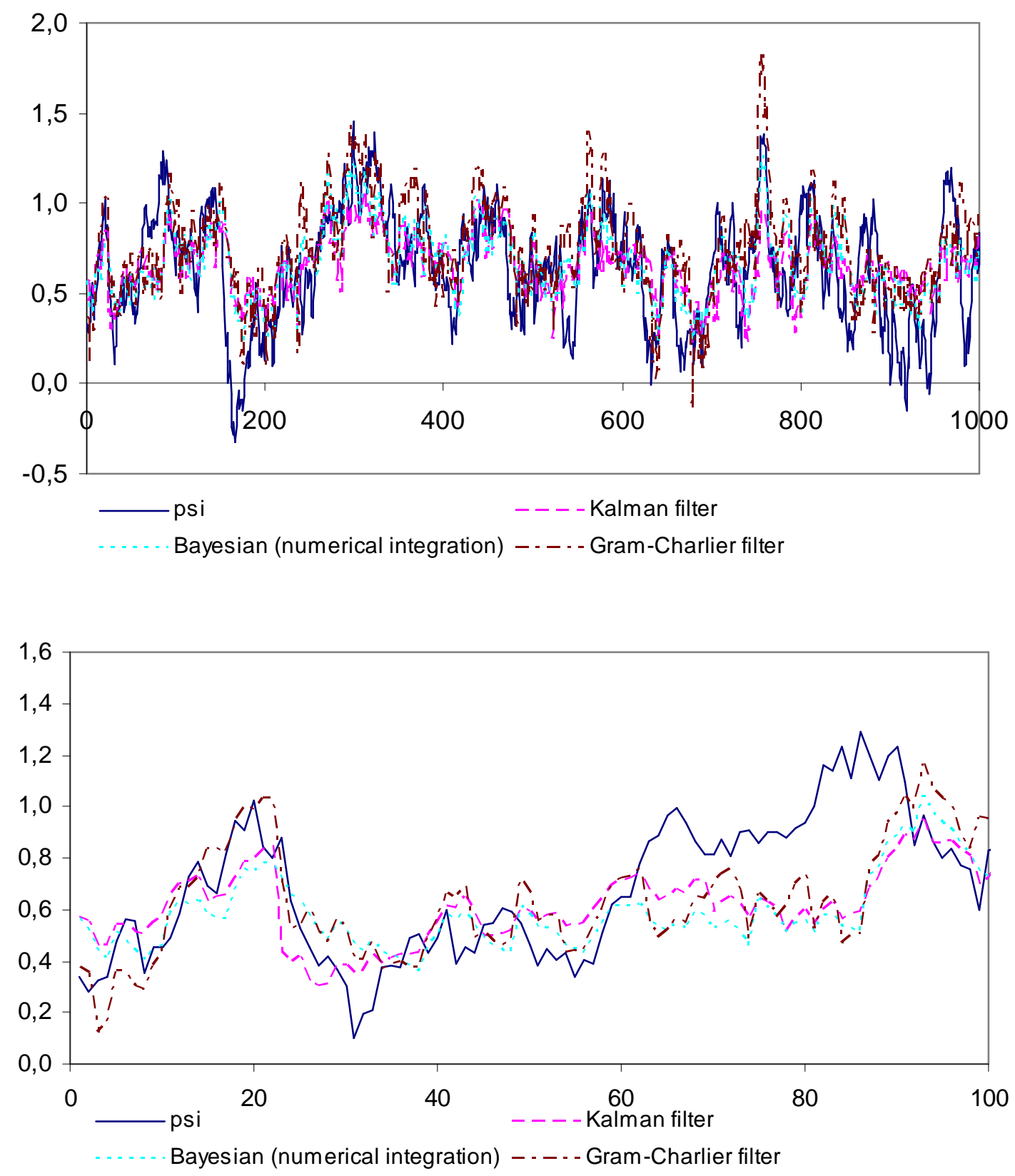

Figura 7.10: Variável latente $\psi$ ("psi") da distribuição exponencial com parâmetros $\delta=$ 0.033, $\phi=0.95, \sigma_{\eta}=0.1, \lambda=1$ (distribuição exponencial), e filtragem obtida através de procedimento de integração numérica ("Bayesian"), através do filtro de Kalman e através do filtro de Gram-Charlier.

A figura de cima é de uma amostra de tamanho 1 000, enquanto que a figura de baixo é uma ampliação dos 100 primeiros dados. 

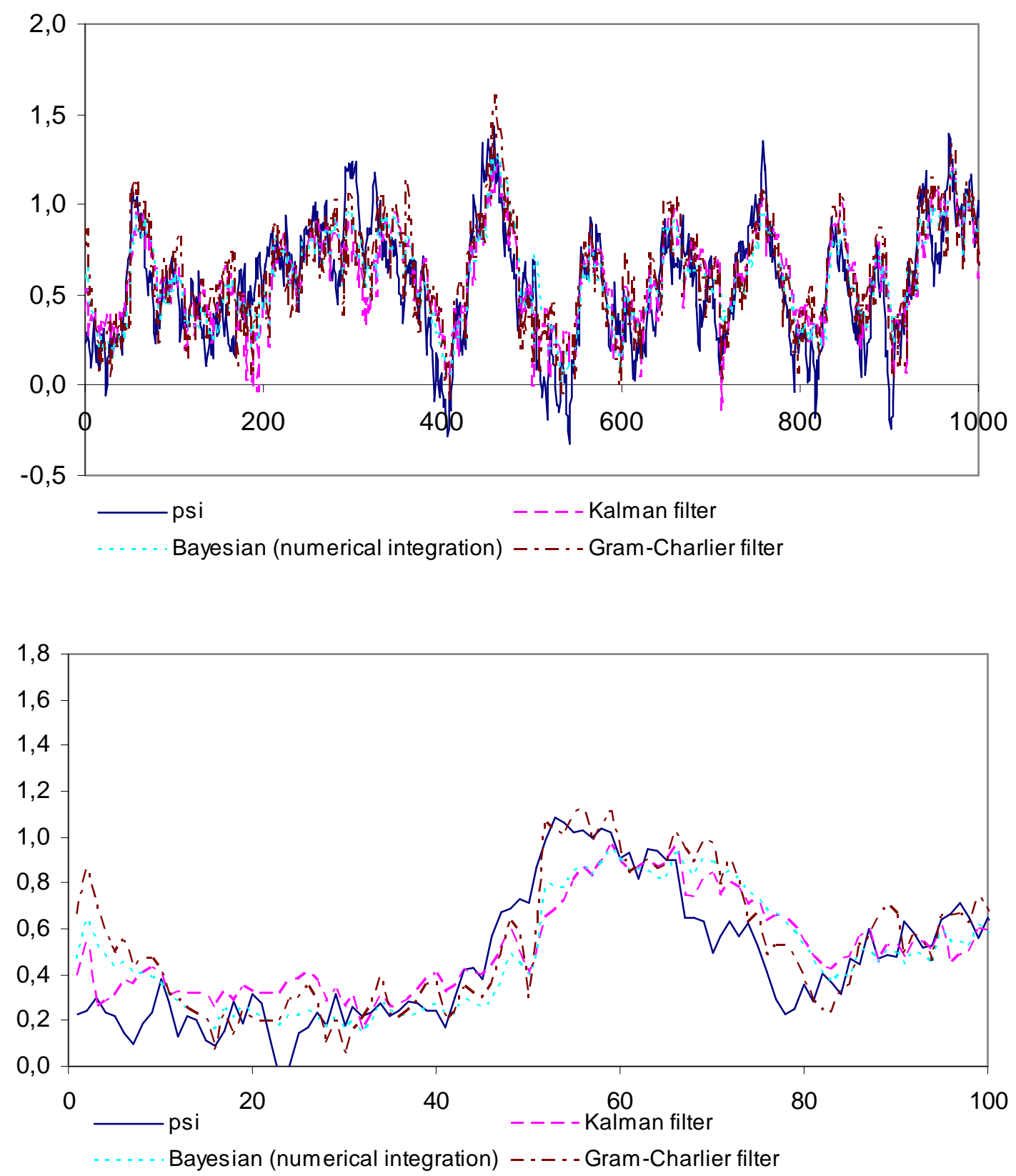

Figura 7.11: Variável latente $\psi$ ("psi") da distribuição Weibull com parâmetros $\delta=0.033$, $\phi=0.95, \sigma_{\eta}=0.1, \lambda=2$, e filtragem obtida através de procedimento de integração numérica ("Bayesian"), através do filtro de Kalman e através do filtro de Gram-Charlier.

A figura de cima é de uma amostra de tamanho 1 000, enquanto que a figura de baixo é uma ampliação dos 100 primeiros dados. 

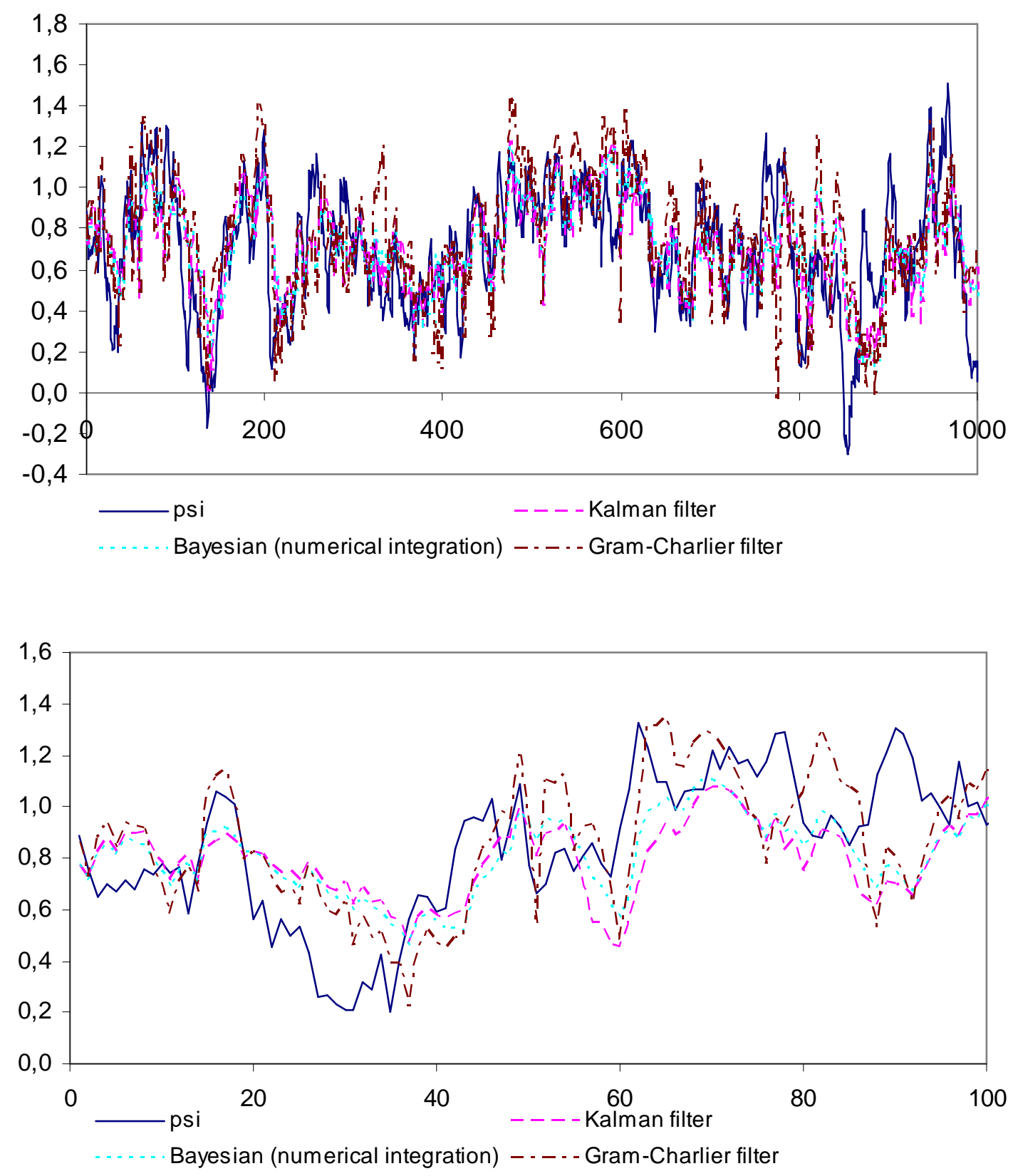

Figura 7.12: Variável latente $\psi$ ("psi") da distribuição gama com parâmetros $\delta=0.033$, $\phi=0.95, \sigma_{\eta}=0.1, \lambda=2$, e filtragem obtida através de procedimento de integração numérica ("Bayesian"), através do filtro de Kalman e através do filtro de Gram-Charlier.

A figura de cima é de uma amostra de tamanho 1 000, enquanto que a figura de baixo é uma ampliação dos 100 primeiros dados. 

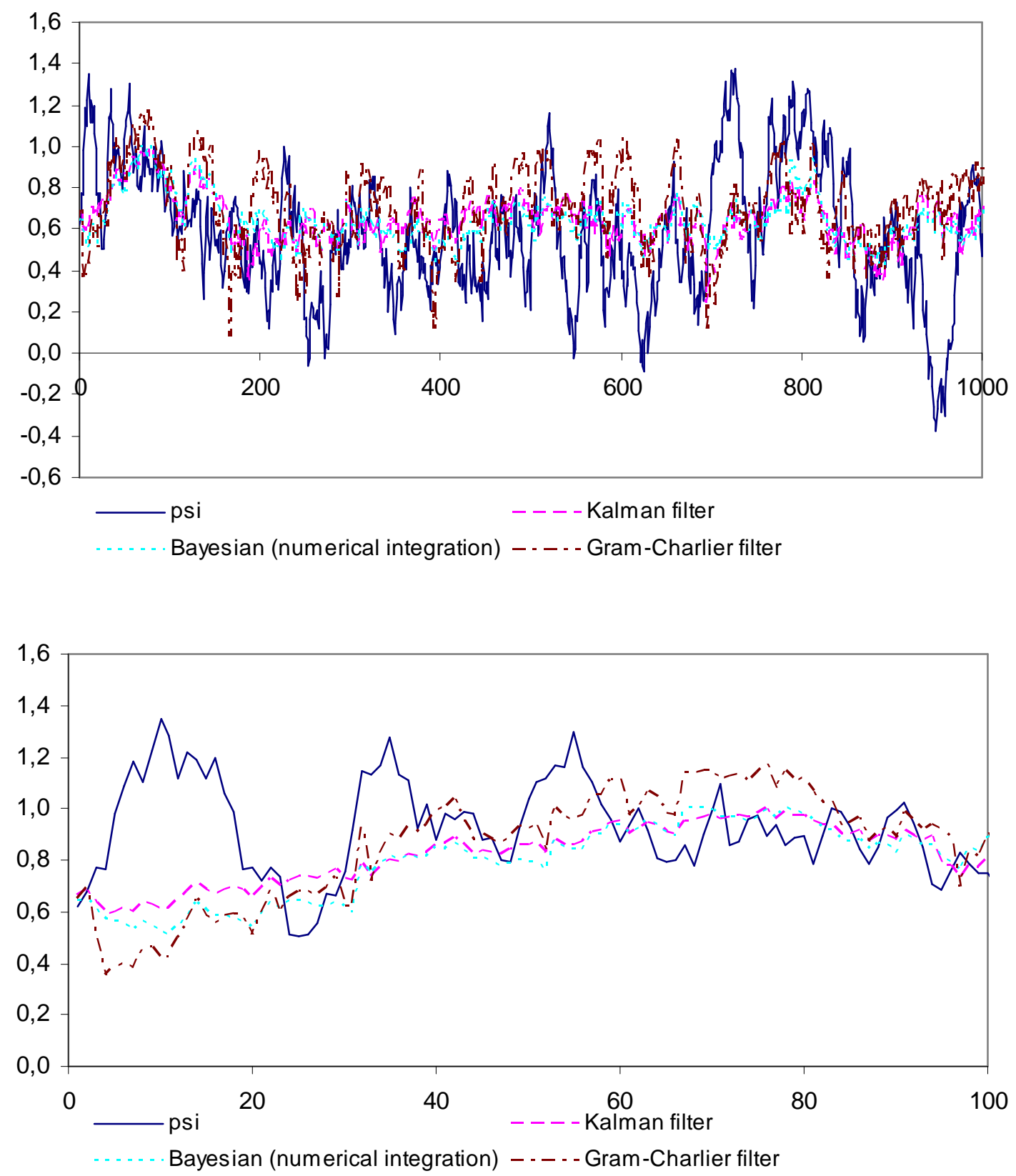

Figura 7.13: Variável latente $\psi$ ("psi") da distribuição Weibull com parâmetros $\delta=0.033$, $\phi=0.95, \sigma_{\eta}=0.1, \lambda=0.5$, e filtragem obtida através de procedimento de integração numérica ("Bayesian"), através do filtro de Kalman e através do filtro de Gram-Charlier. A figura de cima é de uma amostra de tamanho 1 000, enquanto que a figura de baixo é uma ampliação dos 100 primeiros dados. 

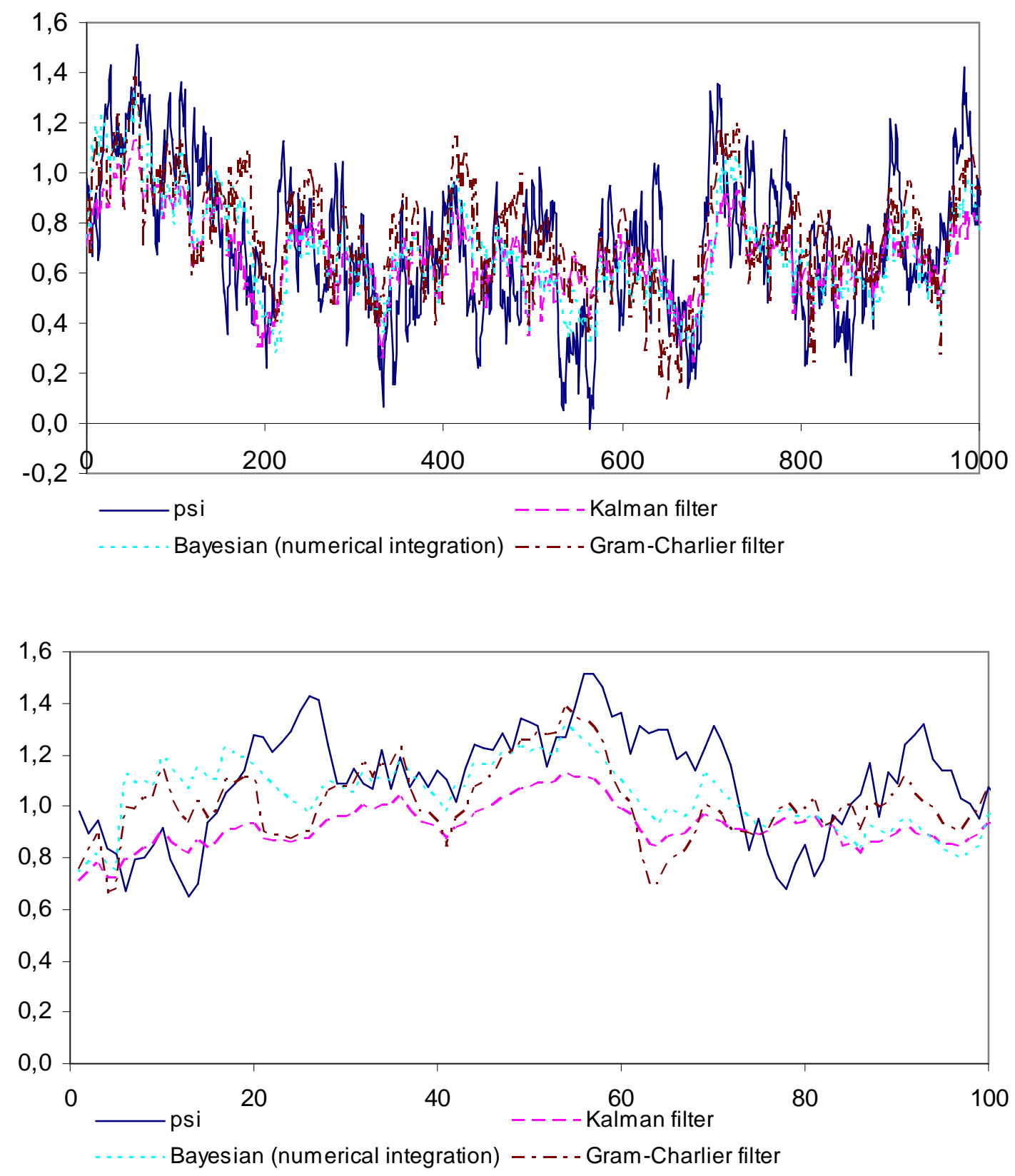

Figura 7.14: Variável latente $\psi$ ("psi") da distribuição gama com parâmetros $\delta=0.033$, $\phi=0.95, \sigma_{\eta}=0.1, \lambda=0.5$, e filtragem obtida através de procedimento de integração numérica ("Bayesian"), através do filtro de Kalman e através do filtro de Gram-Charlier. A figura de cima é de uma amostra de tamanho 1 000, enquanto que a figura de baixo é uma ampliação dos 100 primeiros dados. 


\subsection{Sumário}

Neste capítulo aplicamos os resultados dos capítulos anteriores a conjuntos de dados obtidos por simulação, conhecidos os parâmetros do processo SCD. De uma maneira geral observamos que os algoritmos CECF e DECF apresentaram menor desvio absoluto médio do que o algoritmo QML. Entretanto, para distribuições de cauda muito pesada $(\lambda=0.5)$ todos os três estimadores apresentaram distribuições bastante distantes da normal, mesmo em amostras de tamanho 10000 .

Com relação aos métodos de filtragem para a variável latente do processo SCD, observamos que o filtro Bayesiano foi o que apresentou melhor desempenho em todas as medidas: menor erro quadrático médio, menor desvio absoluto médio e menor mediana dos desvios absolutos.

No próximo capítulo vamos aplicar os métodos de estimação em um conjunto de dados reais. 


\section{Capítulo 8}

\section{Aplicação empírica}

Neste capítulo vamos procurar aplicar os métodos desenvolvidos nos capítulos anteriores para um conjunto de dados reais. Para isso utilizamos os dados disponibilizados por Eric Zivot na sua webpage http://faculty.washington.edu/ezivot/ezresearch.htm, sob o item "Analysis of High-Frequency Financial Data with S-Plus". Uma descrição pormenorizada dos dados, por sua vez, dados pode ser encontrada em Yan e Zivot (2003).

\subsection{Descrição e tratamento dos dados}

Os dados consistem em dados de negociação registrados pelo sistema TAQ (Trade and Quotes) da bolsa de Nova York (NYSE - New York Stock Exchange) das ações da General Eletric (GE) e Microsoft (MSFT) entre 01/05/1997 e 15/05/1997, e dados de cotações de compra (bid) e venda (ask) da taxa de câmbio spot USD/EUR (dólares americanos por Euro) entre 11/03/2001 e 17/03/2001.

Como observado por Yan e Zivot (2003) os dados de alta-freqüência contém uma série de problemas que precisam ser resolvidos antes de iniciarmos a análise. Aqui procuramos seguir, na medida do possível, os procedimentos utilizados por Bauwens e Veredas (2004).

Inicialmente eliminamos os registros de GE e MSFT fora do horário do pregão da NYSE, que funciona entre 9:30 (34 200 s) e 16:00 (57 $600 \mathrm{~s})$.

Em seguida, tratamos os registros com horários de negociação iguais, isto é, de duração 0 . Bauwens e Veredas (2004) eliminam os registros de mesmo horário, sob a hipótese que este efeito decorreria de negociações "em bloco" (block trades), isto é, um lote grande é particionado em lotes menores para facilitar a negociação, gerando várias transações com mesmo horário. Salientamos, entretanto, que o problema real é que a menor unidade de tempo do sistema TAQ é o segundo, mas diversas operações acontecem em intervalos menores que 1 segundo, principalmente nestas ações, que são extremamente líquidas. Aqui neste trabalho não vamos tratar explicitamente este problema de "truncamento" dos dados, e vamos prosseguir a análise utilizando o procedimento adotado por Bauwens e Veredas (2004).

Para o caso de USD/EUR, obtivemos os preços médios entre os preços de compra e venda, e eliminamos os dados relativos ao sábado (17/03/2001), que correspondiam a apenas uma 
pequena fração do dia.

Para a GE notamos que no dia 12/03/1997 houve um evento de split de ações na proporção de 1:2, isto é, cada ação foi desmembrada em 2 , e naturalmente o preço caiu pela metade. Assim, para obter uma série "contínua", dividimos por 2 os preços anteriores a 12/03/2001 e multiplicamos por 2 os tamanhos de lote.

Bauwens e Veredas (2004) definem três tipos de duração:

- Negócio (trade duration): é o intervalo de tempo entre operações consecutivas.

- Preço (price duration): é o intervalo de tempo mínimo necessário para observar uma mudança de preço não menor do que um certo valor. Como explicado em Engle e Russell (1998), esta é uma medida da volatilidade instantânea do mercado. Para os dados de GE e MSFT utilizamos o valor de US\$ 0.125 para mudança de preço, e para os dados de USD/EUR utilizamos US\$0.0005 (moda do bid/ask spread).

- Volume (volume duration): é o intervalo de tempo para que o volume total negociado não seja menor do que 25000 ações. Esta é uma medida indireta da liquidez do papel, pois indica o tempo necessário para negociar um certo volume de ações $(25$ 000, no caso). Para os dados USD/EUR não é possível calcular esta medida, pois não dispomos dos dados de negociação.

Bauwens e Veredas (2004) observam que os dados apresentam dois tipo de variação "sazonal" (sistemática): uma variação que ocorre durante o dia, chamado efeito "diurno", e uma variação que ocorre entre os dias, chamada efeito "dia da semana".

O conhecido o efeito "diurno" nos dados de alta freqüência é resultante da variação sistemática da atividade de mercado durante o dia. Vamos tratar este efeito decompondo a duração em duas partes: uma estocástica (a ser explicada pelo modelo SCD) e uma determinística, determinada pelo efeito "diurno". Para eliminar este efeito vamos seguir Bauwens e Veredas (2004) definindo

$$
d_{i}=\frac{D_{i}}{\phi\left(\tau_{i}\right)}
$$

como a duração ajustada (estocástica), sendo $D_{i}$ a duração original e $\phi\left(\tau_{i}\right)$ o efeito diurno em $\tau_{i}$. O efeito diurno é calculado através de uma regressão não-paramétrica da duração original contra o instante do dia através do estimador de Nadaraya-Watson

$$
\phi(\tau)=\frac{\sum_{i=1}^{n} K\left(\frac{\tau-t_{i}}{h}\right) D_{i}}{\sum_{i=1}^{n} K\left(\frac{\tau-t_{i}}{h}\right)}
$$

onde $h$ é um parâmetro para controle da suavização, chamado de largura de banda (bandwidth), e $K($.) é uma função kernel apropriada. Utilizamos em todas as amostras o kernel normal (Gaussiano)

$$
K(u)=\frac{1}{\sqrt{2 \pi}} e^{-\frac{1}{2} u^{2}} .
$$


Tabela 8.1: Resumo dos dados das amostras de durações dessazonalizadas de GE, Microsoft e USD/EUR. Veja o texto para mais detalhes da base de dados e respectivo tratamento.

\begin{tabular}{l|rrr|rrr|rr}
\hline & \multicolumn{4}{|c|}{ Trade } & \multicolumn{3}{c|}{ Price } & \multicolumn{2}{c}{ Volume } \\
\cline { 2 - 9 } & \multicolumn{1}{|c}{ GE } & \multicolumn{1}{c|}{ Microsoft } & USD/EUR & \multicolumn{1}{|c}{ GE } & Microsoft & USD/EUR & GE & Microsoft \\
\hline Amostra & 38,670 & 68,771 & 101,171 & 819 & 5,358 & 461 & 2,090 & 3,150 \\
Mediana & 0.531 & 0.602 & 0.616 & 0.079 & 0.188 & 0.581 & 0.781 & 0.758 \\
Média & 0.987 & 0.976 & 0.998 & 1.000 & 0.953 & 0.988 & 0.973 & 0.968 \\
Desv.pad. & 1.352 & 1.130 & 0.972 & 2.637 & 2.212 & 1.188 & 0.734 & 0.756 \\
Assimetria & 6.518 & 4.337 & 9.659 & 5.650 & 6.820 & 2.399 & 1.521 & 2.264 \\
Curtose & 173.871 & 38.745 & 378.760 & 40.757 & 83.251 & 7.978 & 3.188 & 7.832 \\
Min & 0.126 & 0.198 & 0.023 & 0.003 & 0.014 & 0.001 & 0.007 & 0.009 \\
Max & 67.907 & 30.056 & 67.862 & 30.251 & 45.296 & 9.014 & 4.923 & 6.574 \\
Bandwidth & 3,507 & 3,510 & 1,000 & 3,507 & 3,510 & 1,000 & 3,507 & 3,510 \\
\hline
\end{tabular}

Para os dados da NYSE utilizamos largura de banda dada pela sugestão do EViews, $h=0.15\left(T_{\max }-T_{\min }\right)$, sendo que $T_{\min }=34200$, correspondente a 9:30, e $T_{\max }=57600$, correspondente a 16:00, o que fornece o valor de $h=3510$. Já para os dados de USD/EUR utilizamos largura de banda $h=1000$ com o objetivo de tentar capturar mais detalhes (o período de amostragem é de $24 \mathrm{~h})$.

Bauwens e Veredas (2004) observam também uma "sazonalidade" devido ao dia da semana, calculando desta forma um fator diurno diferente para cada dia da semana. Na nossa amostra acreditamos que este efeito deva ser mais pronunciado nos dados de USD/EUR, já que este mercado é operado no mundo inteiro. Por exemplo, em Londres no domingo podem aparecer operações decorrentes das operações em Tokyo, onde já é segunda-feira. Infelizmente como a nossa base de dados contém apenas 2 semanas de dados, não é possível calcular este efeito. Assim, vamos assumir que o efeito diurno seja igual para todos os dias da semana.

A tabela 8.1 apresenta um resumo das características das amostras. Nas seções seguintes vamos apresentar os resultados das estimações obtidos para a duração de volume da GE e da Microsoft, pois são os processos que parecem ser mais bem comportados (estacionários). Os resultados para as durações de negócio (altamente não estacionária) e de preço encontram-se no apêndice $\underline{\mathrm{H}}$. 


\subsection{Duração de volume da GE}

Podemos notar pelo exame da figura 8.1 o perfil característico de "U" invertido do efeito diurno da duração de volume.

Os parâmetros do processo SCD obtidos com os diferente métodos de estimação (QML, CECF e DECF) encontram-se ilustrados na tabela 8.2, considerando-se as distribuições Weibull e gama para os erros.

$\mathrm{Na}$ figura 8.2 apresentamos a função característica empírica e as funções características para distribuição gama, com as estimativas dos métodos QML e CECF. Analisando a tabela 8.2. note como para a distribuição Weibull os três métodos apresentam resultados muito parecidos, enquanto que para distribuição gama o método DECF parece parece estimar melhor (comparando com os resultados para a distribuição Weibull) que o método QML, que subestima a persistência do processo ( $\phi=0.805$ para QML contra 0.986 do DECF), e o método CECF, que superestima ligeiramente a persistência (0.996 do CECF).

A figura 8.3 apresenta os resultados dos diferentes filtros, aplicados à estimativa obtida com o método CECF, enquanto a figura 8.4 apresenta os gráficos de quantis ("QQ-plots") das estimativas dos resíduos (tanto da equação de observação, quanto da equação de estado) obtidas com os diferentes filtros, e um gráfico da autocorrelação dos resíduos utilizando o filtro de Kalman. Note que o filtro de Kalman foi o que apresentou resíduos mais bem comportados, tanto para para a equação de observação, quanto para equação de estado, embora alguma autocorrelação ainda permaneça nos mesmos. 

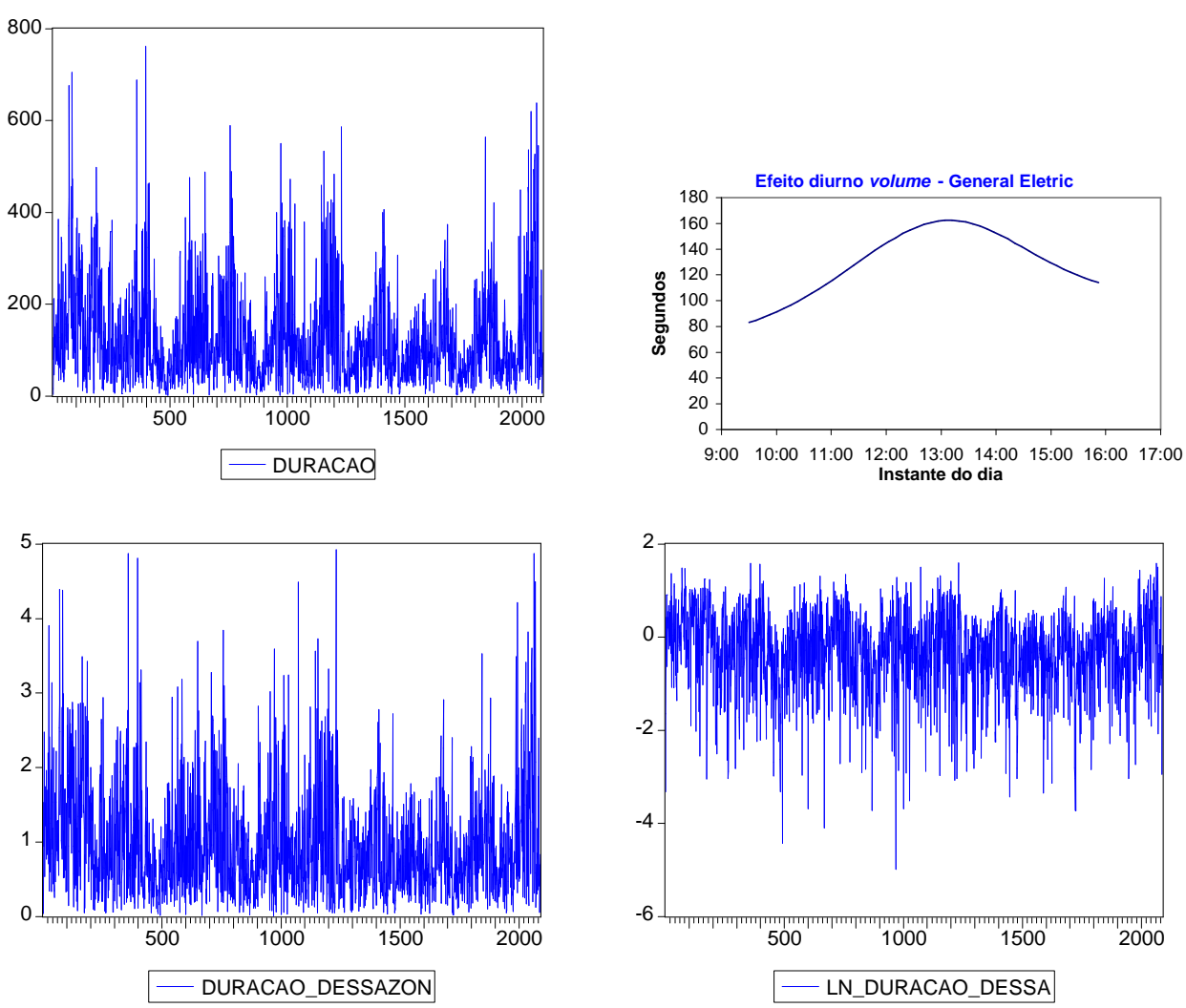

Figura 8.1: Dados da GE:

Cima: duração de volume (volume duration) e respectiva componente diurna. Baixo: duração de volume dessazonalizada e respectivo logaritmo.

\begin{tabular}{|c|c|c|c|c|c|c|}
\hline \multirow[t]{2}{*}{ Parâmetro } & \multicolumn{3}{|c|}{ Weibull } & \multicolumn{3}{|c|}{ Gamma } \\
\hline & QML & CECF & DECF & QML & CECF & DECF \\
\hline$\delta$ & 0.001 & 0.001 & 0.001 & -0.020 & 0.000 & -0.001 \\
\hline$\phi$ & 0.987 & 0.987 & 0.986 & 0.805 & 0.996 & 0.986 \\
\hline$\sigma_{\eta}$ & 0.076 & 0.082 & 0.089 & 0.277 & 0.037 & 0.072 \\
\hline$\lambda$ & 1.976 & 1.956 & 1.915 & 2.296 & 2.381 & 2.746 \\
\hline Tempo (s) & 11.1 & 12.4 & 877.9 & 11.1 & 293.3 & 844.4 \\
\hline
\end{tabular}

Tabela 8.2: Estimação dos parâmetros do modelo SCD para a duração de volume (volume duration) da GE, considerando distribuições Weibull e gama para os erros. 

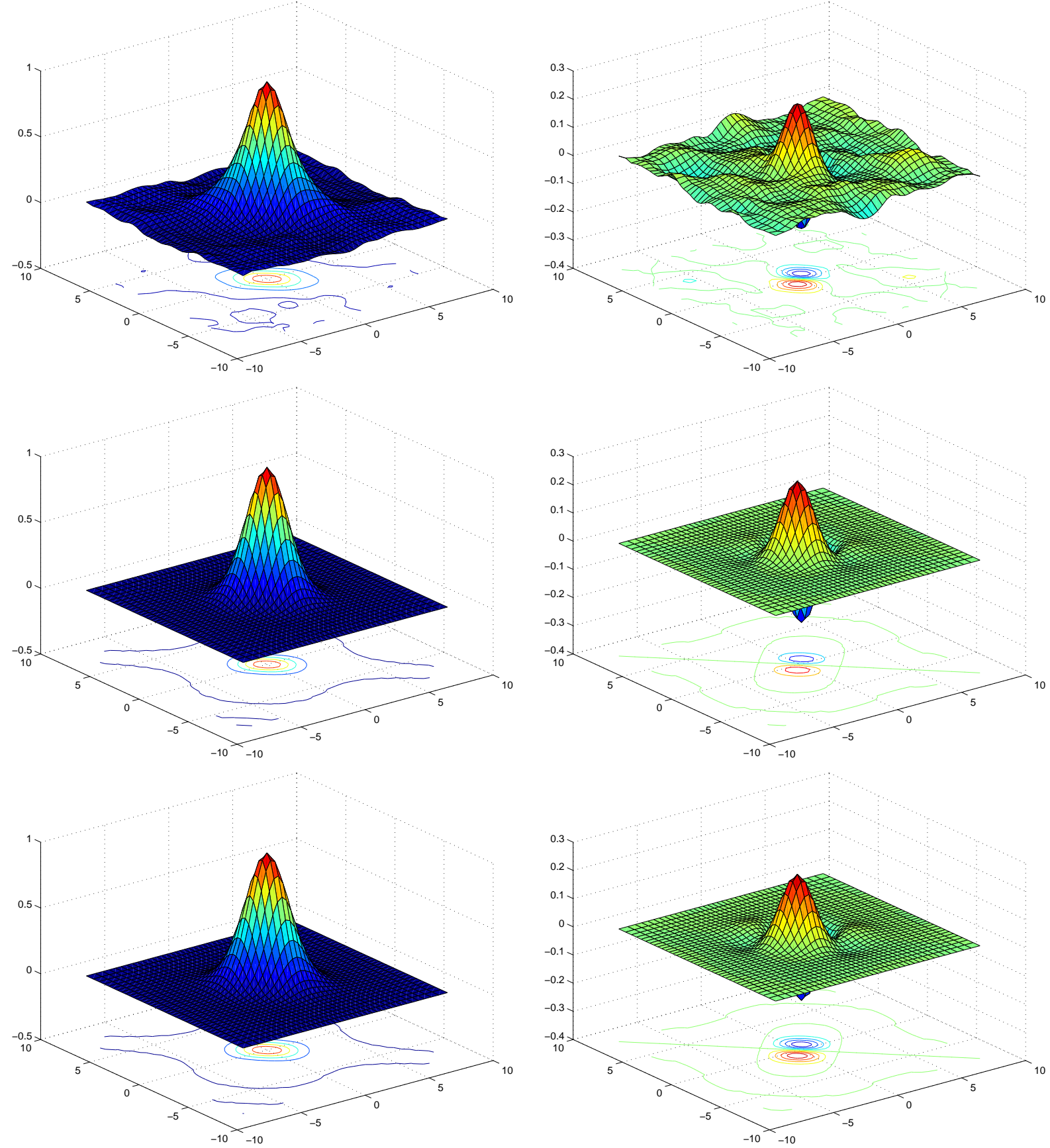

Figura 8.2: Partes real (esquerda) e imaginária (direita) da função característica empírica (cima) e das funções características com distribuição gama para a duração de volume (volume duration) da GE, calculadas em $\theta_{Q M L}$ (meio) e $\theta_{C E C F}$ (baixo), para a malha de 39 nós da quadratura de Gauss-Hermite. 

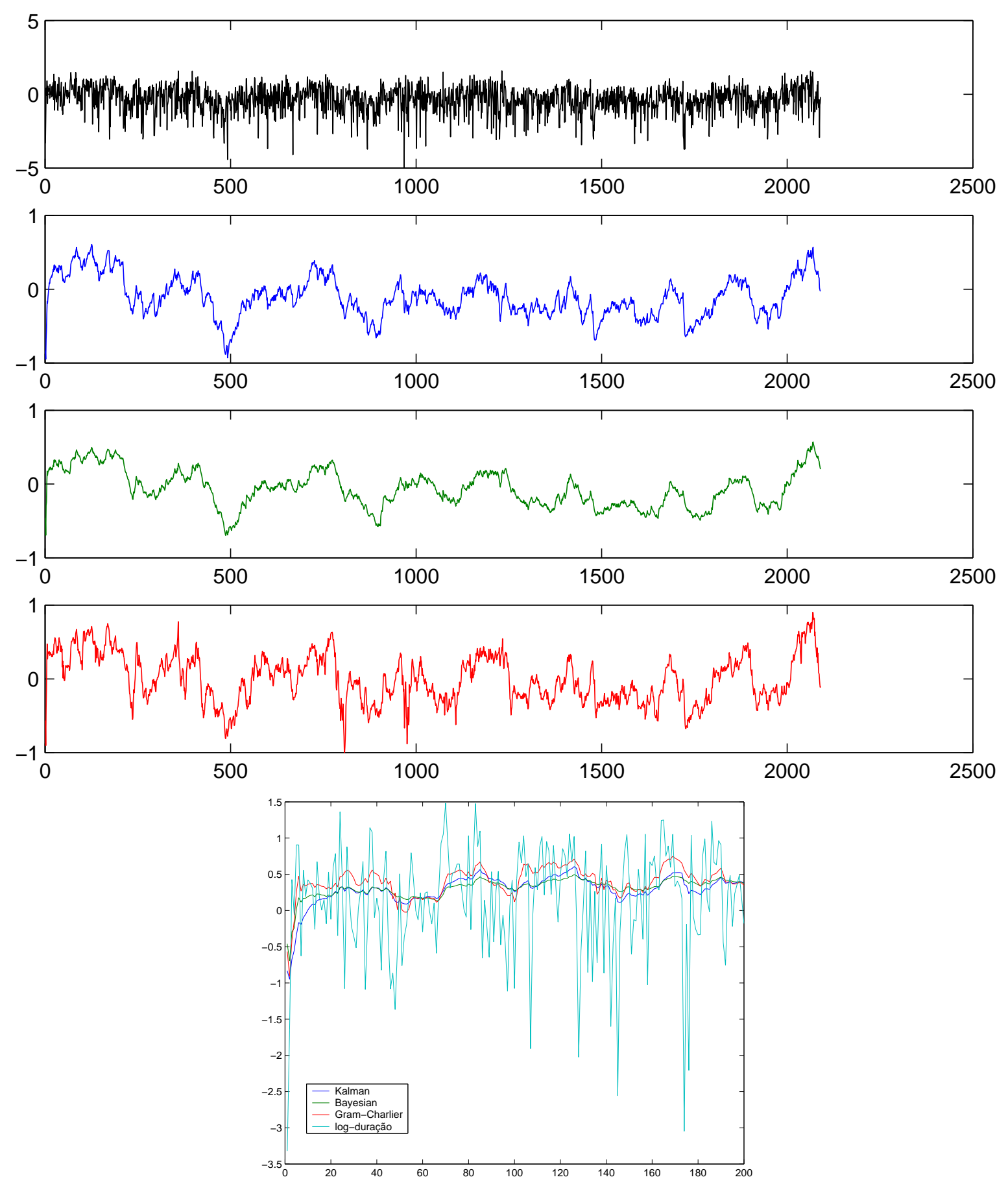

Figura 8.3: Estimativa da variável latente para a duração de volume (volume duration) da GE.

Cima: de cima para baixo temos os seguinte filtros calculados em $\theta_{C E C F}$ : (1) Log-durações; (2) filtro de Kalman; (3) filtro Bayesiano; (4) filtro Gram-Charlier.

Baixo: primeiras 200 observações dos filtros. 

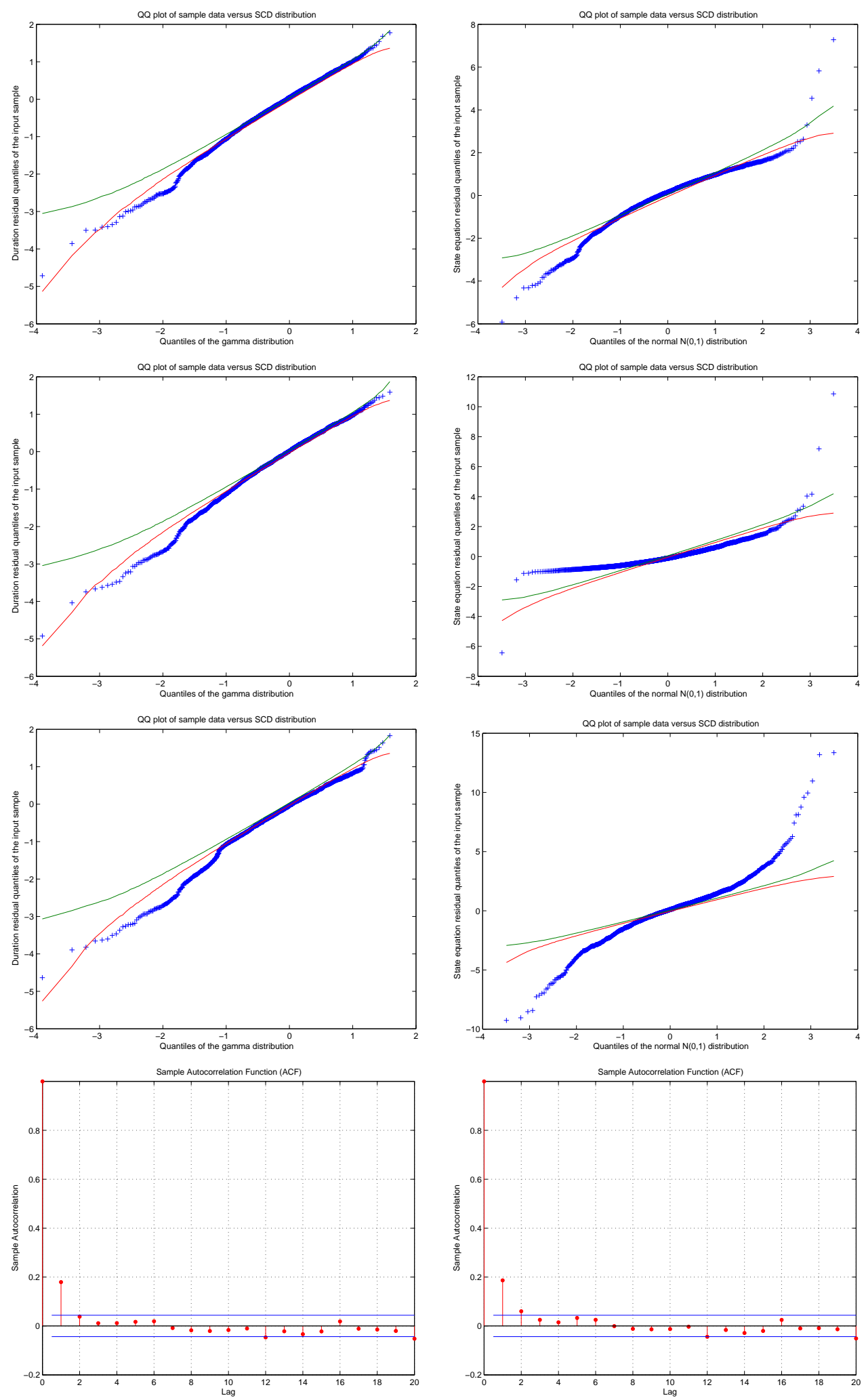

Figura 8.4: De cima para baixo temos os gráficos de quantis dos resíduos obtidos com os filtros calculados em $\theta_{C E C F}$ com distribuição gama para a duração de volume da GE: (1) filtro de Kalman; (2) filtro Bayesiano; (3) filtro Gram-Charlier; (4) função de autocorrelação dos resíduos do filtro de Kalman.

À esquerda vemos os resíduos da equação de observação e à direita os resíduos da equação de estado. As linhas representam os limites do intervalo de $95 \%$ de confiança, obtidos através de simulação. 


\subsection{Duração de volume da Microsoft}

A série de duração de volume, de duração dessazonalizada, seu logaritmo e o efeito diurno para a Microsoft estão ilustradas na figura 8.5. Uma inspeção da figura de duração dessazonalizada parece indicar um aumento sistemático das durações, o que tornaria a série não estacionária. Mesmo assim, vamos prosseguir com a análise como na seção anterior.

Os parâmetros do processo SCD obtidos com os diferente métodos de estimação (QML, CECF e DECF) encontram-se ilustrados na tabela 8.3, considerando-se as distribuições Weibull e gama para os erros.

Na figura 8.6 apresentamos a função característica empírica e as funções características para distribuição gama, com as estimativas do método QML e CECF. Note que, tanto para a distribuição Weibull quanto para a distribuição gama, os três métodos apresentam resultados razoavelmente parecidos.

A figura 8.7 apresenta os resultados dos diferentes filtros, aplicados à estimativa obtida com o método CECF, enquanto a figura 8.8 apresenta os gráficos de quantis ("QQ-plots") das estimativas dos resíduos (tanto da equação de observação, quanto da equação de estado) obtidas com os diferentes filtros, e um gráfico da autocorrelação dos resíduos utilizando o filtro de Kalman. Note que o filtro de Kalman foi o que apresentou resíduos mais bem comportados para a equação de observação, mas o filtro de Gram-Charlier apresenta resíduos mais bem comportados para a equação de estado. Também os resíduos obtidos parecem limpos de autocorrelação. 

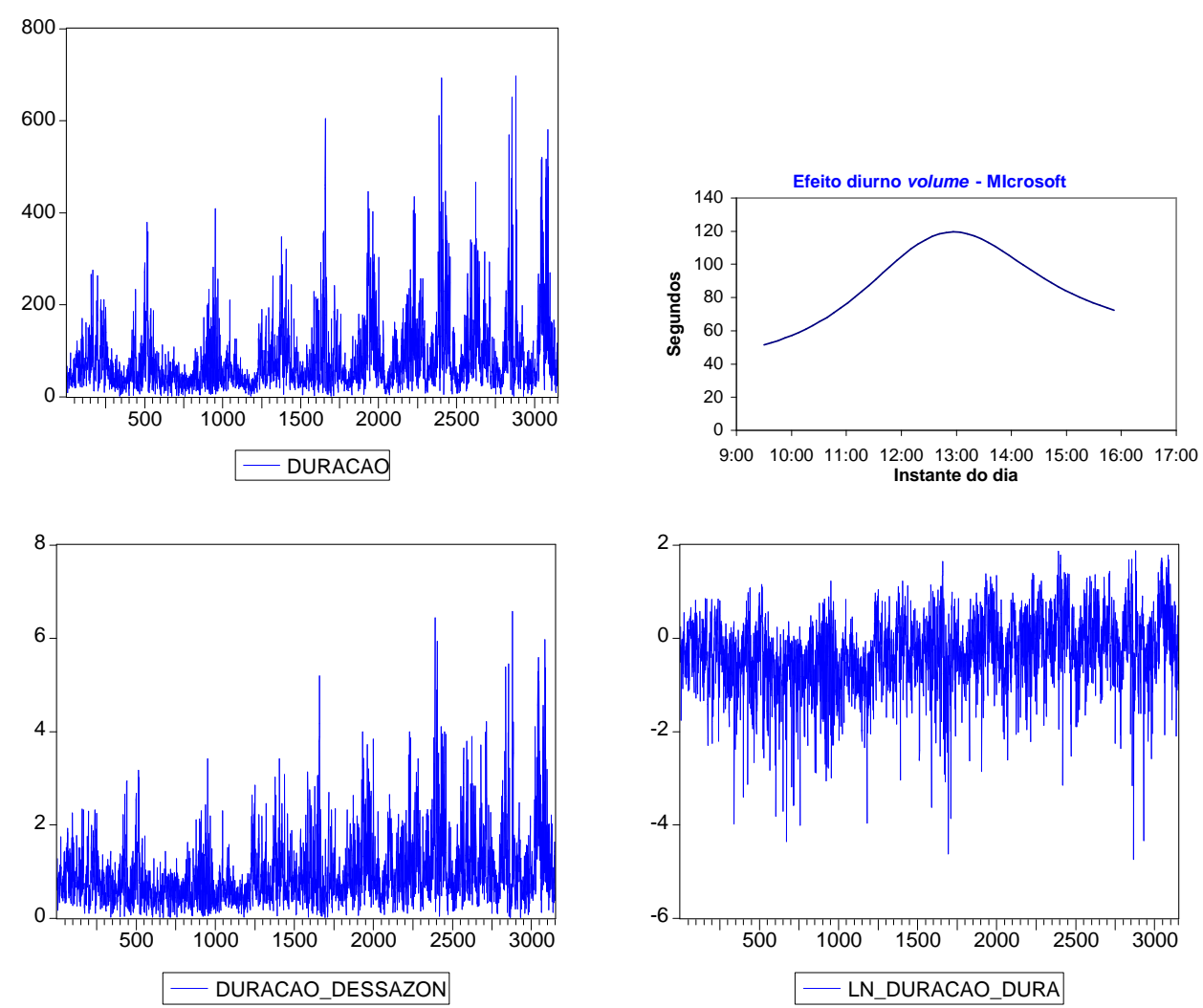

Figura 8.5: Dados da Microsoft:

Cima: duração de volume (volume duration) e respectiva componente diurna.

Baixo: duração de volume dessazonalizada e respectivo logaritmo.

\begin{tabular}{|c|c|c|c|c|c|c|}
\hline \multirow[t]{2}{*}{ Parâmetro } & \multicolumn{3}{|c|}{ Weibull } & \multicolumn{3}{|c|}{ Gamma } \\
\hline & QML & CECF & DECF & QML & CECF & DECF \\
\hline$\delta$ & -0.010 & -0.012 & -0.019 & -0.009 & -0.010 & -0.009 \\
\hline$\phi$ & 0.928 & 0.928 & 0.905 & 0.928 & 0.928 & 0.947 \\
\hline$\sigma_{\eta}$ & 0.175 & 0.181 & 0.207 & 0.175 & 0.180 & 0.151 \\
\hline$\lambda$ & 2.069 & 2.296 & 2.454 & 3.071 & 3.814 & 4.332 \\
\hline Tempo (s) & 0.6 & 8.9 & 857.7 & 0.5 & 9.4 & 874.0 \\
\hline
\end{tabular}

Tabela 8.3: Estimação dos parâmetros do modelo SCD para a duração de volume (volume duration) da Microsoft, considerando distribuições Weibull e gama para os erros. 

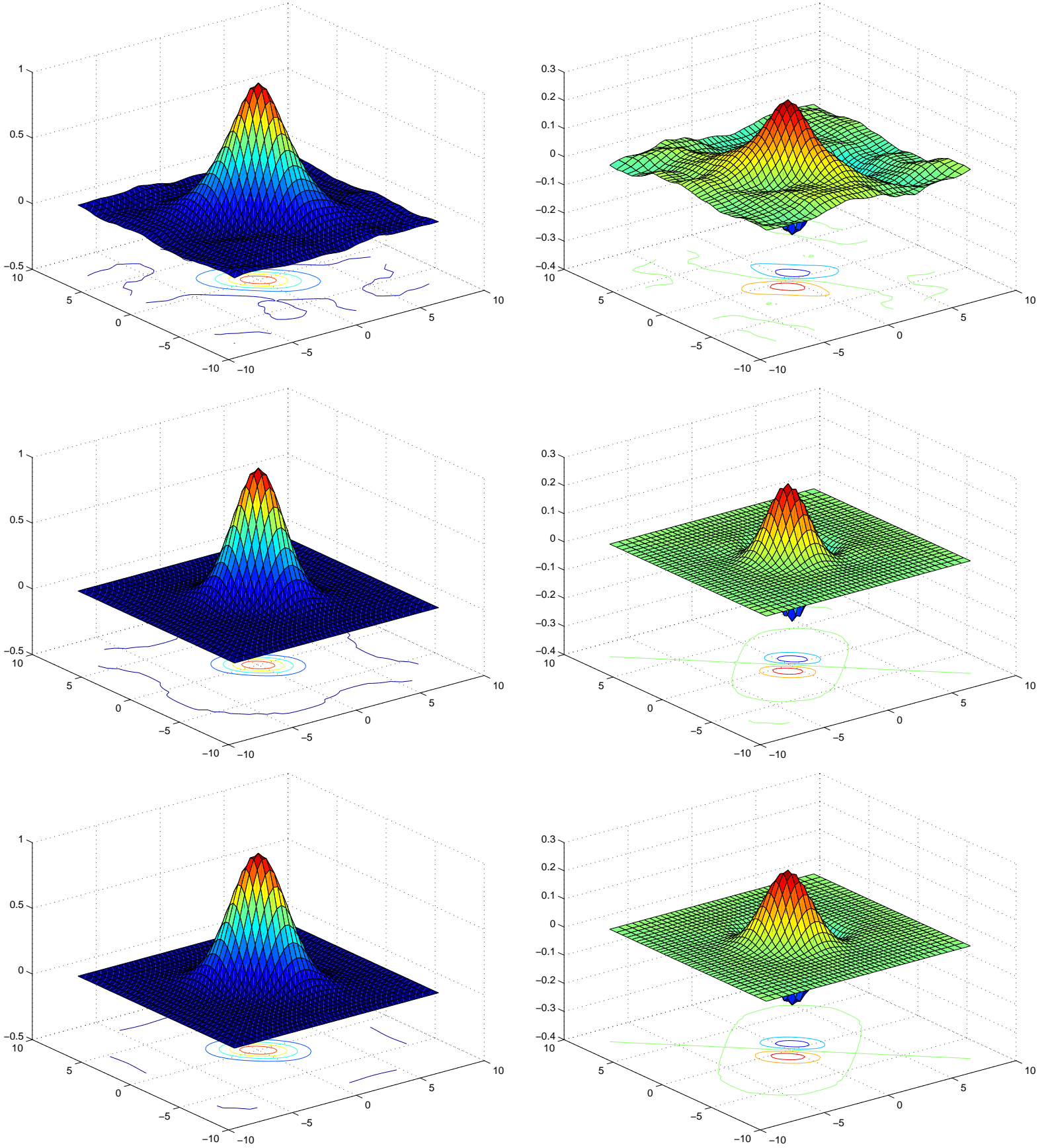

Figura 8.6: Partes real (esquerda) e imaginária (direita) da função característica empírica (cima) e das funções características com distribuição gama para a duração de volume (volume duration) da Microsoft, calculadas em $\theta_{Q M L}$ (meio) e $\theta_{C E C F}$ (baixo), para a malha de 39 nós da quadratura de Gauss-Hermite. 

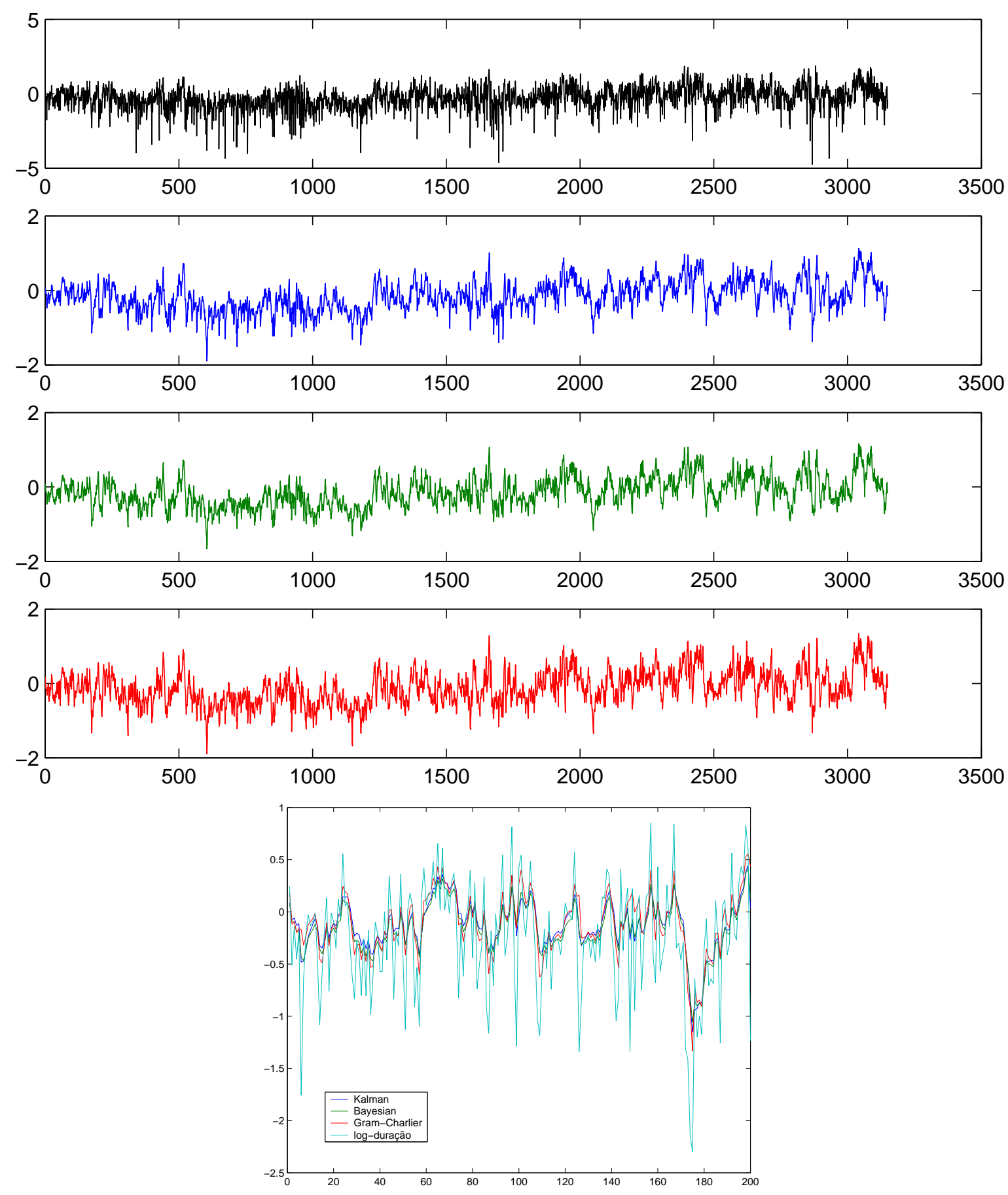

Figura 8.7: Estimativa da variável latente para a duração de volume (volume duration) da Microsoft.

Cima: de cima para baixo temos os seguinte filtros calculados em $\theta_{C E C F}$ : (1) Log-durações; (2) filtro de Kalman; (3) filtro Bayesiano; (4) filtro Gram-Charlier.

Baixo: primeiras 200 observações dos filtros. 

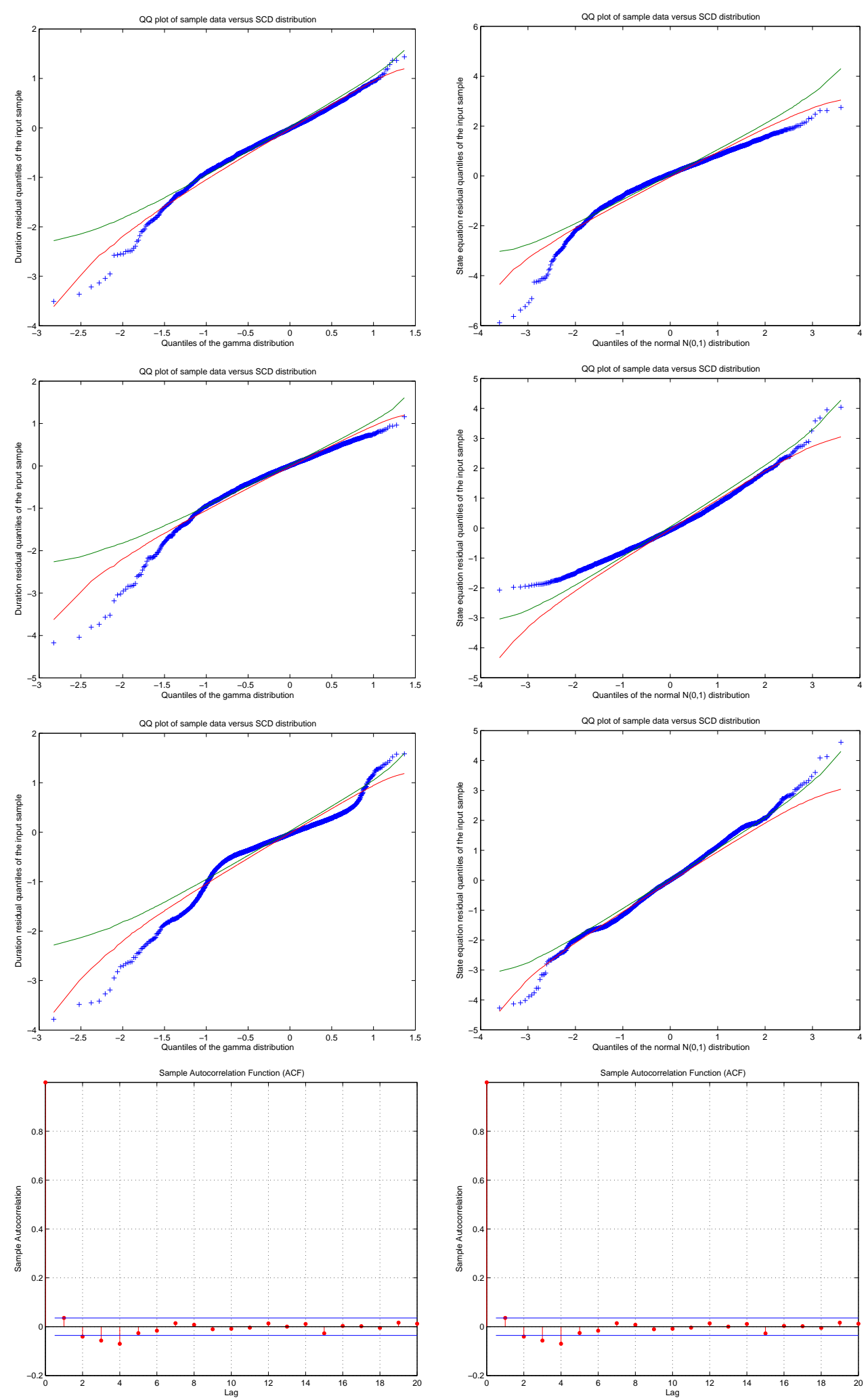

Figura 8.8: De cima para baixo temos os gráficos de quantis dos resíduos obtidos com os filtros calculados em $\theta_{C E C F}$ com distribuição gama para a duração de volume da Microsoft: (1) filtro de Kalman; (2) filtro Bayesiano; (3) filtro Gram-Charlier; (4) função de autocorrelação dos resíduos do filtro de Kalman.

À esquerda vemos os resíduos da equação de observação e à direita os resíduos da equação de estado. As linhas representam os limites do intervalo de $95 \%$ de confiança, obtidos através de simulação. 


\subsection{Sumário}

Nesta seção aplicamos os resultados desenvolvidos nos capítulos anteriores para séries reais de dados de alta frequência. Em particular estimamos os parâmetros do modelo SCD aplicado às séries de duração de volume de General Eletric e de Microsoft, e relatamos os resultados obtidos. 


\section{Capítulo 9}

\section{Conclusão}

A função característica empírica (ECF) tem sido utilizada em problemas estimação nos quais é difícil aplicar-se o método da máxima verossimilhança, tais como:

- distribuições estáveis;

- processos afins de difusão com saltos (ver por exemplo Duffie et al. (2000));

- mistura de normais

- processos com variáveis latente, como o modelo de volatilidade estocástica.

Neste trabalho utilizamos a ECF para estimação do modelo de duração condicional estocástica (SCD), que é um modelo análogo ao modelo de volatilidade estocástica. Para isso, obtivemos alguns resultados relativos ao processo, e obtivemos também as distribuições assintóticas dos estimadores CECF e DECF.

De uma maneira geral, tanto o estimador CECF quanto o estimador DECF parecem melhorar a distribuição do estimador QML, usado como ponto inicial. Entretanto a convergência para a distribuição assintótica normal parece muito lenta, mesmo para amostras de tamanho 10 000. Também observamos lenta convergência dos estimadores das matrizes de covariâncias dos estimadores CECF e DECF, causada pela convergência da matriz $\Omega_{n}$.

Após a obtenção dos parâmetros do modelo SCD, obtivemos estimativas para as variáveis latentes. Para isso implementamos um filtro de Kalman, um filtro Bayesiano obtido através de integração numérica (quadratura), e um filtro baseado na expansão de Gram-Charlier. Observamos nas simulações que o filtro de Kalman, apesar de Gaussiano, é razoavelmente robusto, enquanto que o filtro Bayesiano foi o que apresentou melhor desempenho em todas as medidas (erro quadrático médio, desvio absoluto médio e mediana dos desvios absolutos).

Quanto a possíveis extensões deste trabalho poderiamos indicar, por exemplo:

- Extensão do modelo SCD para ordens maior do que 1, com obtenção da respectiva função poli-característica. 
- Desenvolvimento de análise de diagnóstico do método da ECF, tanto para identificar problemas de amostragem, quanto para identificar problemas de especificação do modelo.

- Desenvolvimento do métodos de integração numérica por métodos MCMC (Markov Chain Monte Carlo) como em Strickland et al. (2005), tanto para a obtenção dos parâmetros do modelo SCD, quanto para filtragem (obtenção das variáveis latentes). 


\section{Apêndice A}

\section{Propriedades de algumas classes de estimadores}

\section{A.1 Propriedades assintóticas dos estimadores extremos}

Um estimador $\widehat{\theta}$ é chamado de estimador extremo ("extremum estimator") se existir uma função escalar $Q_{n}(z, \theta)$ tal que $\widehat{\theta}$ minimize (ou maximize) $Q_{n}(z, \theta)$ sujeito a $\theta \in \Theta \subset \mathbb{R}^{k}$, onde $z=\left(z_{1}, z_{2}, \cdots, z_{n}\right)^{\prime}$, sendo cada $z_{j}$ um vetor de observações na amostra de tamanho $n$. Hayashi (2000) identifica duas classes especiais de estimadores de extremos:

(a) Estimadores M, i.e., do tipo Máxima verossimilhança (Huber (1981), Serfling (1980) ou der Vaart (1998)), são obtidos quando a função objetivo for uma média amostral, i.e.,

$$
Q_{n}(z, \theta)=\frac{1}{n} \sum_{j=1}^{n} m\left(z_{j} ; \theta\right),
$$

onde $m$ é uma função real de $\left(z_{j}, \theta\right)$. Estimadores de máxima verossilhança (ML) e estimadores de mínimos quadrados não lineares (NLS) são dois exemplos de estimadores desta classe.

(b) Estimadores GMM - método generalizado dos momentos (Hansen (1982) ou Hayashi (2000)), são obtidos quando a função objetivo puder ser escrita como

$$
Q_{n}(z, \theta)=g_{n}(z, \theta)^{\prime} W_{n} g_{n}(z, \theta)
$$

com

$$
g_{n}(z, \theta)=\frac{1}{n} \sum_{j=1}^{n} f\left(z_{j}, \theta\right)
$$

um vetor $q \times 1$, e $W_{n}$ uma matriz simétrica positiva definida $q \times q$, possivelmente dependente dos dados, e que define a distância de $g_{n}(z, \theta)$ ao zero. Um exemplo de estimador nesta classe é o estimador DECF. 
Note que existem estimadores extremos que não caem em nenhuma das duas classes acima, como por exemplo o estimador CECF.

Nesta seção vamos apresentar alguns resultados para determinação da distribuição assintótica destes estimadores, conforme estabelecido em Amemiva (1985). Uma discussão interessante sobre as propriedades assintóticas deste estimadores, principalmente da classe GMM, pode ser encontrada também em Singleton (2006). Como basicamente não há diferença essencial entre procedimentos de maximização ou de minimização (basta trocar o sinal da função), vamos considerar apenas o caso da minimização. No que segue vamos enunciar praticamente ipsis verbis os resultados apresentados em Amemiva (1985).

Seja $Q_{n}(Z, \theta)$ a função a ser minimizada, onde $Z=\left(Z_{1}, Z_{2}, \cdots, Z_{n}\right)^{\prime}$ é o conjunto das variáveis aleatórias, e $\theta$ é um vetor $k \times 1$ dos parâmetros que caracterizam a distribuição de $Z$. Denotemos por $\Theta$ o espaço paramétrico de $\theta$, e por $\theta_{0}$ o verdadeiro valor do vetor de parâmetros.

Teorema A.1 (Amemiva (1985), Teor. 4.1.1, pg. 106) Sejam as seguintes hipóteses:

(a) O espaço paramétrico $\Theta$ é um subconjunto compacto do espaço Euclidiano k-dimensional $\left(\mathbb{R}^{k}\right)$. Note que $\theta_{0} \in \Theta$

(b) $Q_{n}(Z, \theta)$ é contínua em $\theta \in \Theta$ para todo $Z$ e é uma função mensurável de $Z$ para todo $\theta \in \Theta$.

(c) $n^{-1} Q_{n}(Z, \theta)$ converge uniformemente em probabilidade em $\theta \in \Theta$ para uma função não estocástica $Q(\theta)$ a medida que $n$ vai para $\infty$, e alcança o único mínimo global em $\theta_{0}$. $A$ continuidade de $Q(\theta)$ decorre das hipóteses.

Defina $\widehat{\theta}_{n}$ o valor que satisfaz

$$
Q_{n}\left(Z, \widehat{\theta}_{n}\right)=\min _{\theta \in \Theta} Q_{n}(Z, \theta)
$$

ou seja,

$$
\widehat{\theta}_{n}=\arg \min _{\theta \in \Theta} Q_{n}(Z, \theta)
$$

Subentende-se que caso $\widehat{\theta}_{n}$ não seja único, escolhemos apropriadamente um deles, de maneira que $\widehat{\theta}_{n}(Z)$ seja uma função mensurável de $Z$. Isto é possivel através de um teorema de Jennrich (1969), pg. 63\%.

Então $\widehat{\theta}_{n}$ converge em probabilidade para $\theta_{0}$.

Prova. Ver Amemiya (1985), teorema 4.1.1, pg. 106.

Teorema A.2 (Existência de raiz consistente, Amemiya (1985), Teor. 4.1.2, pg. 110) Façamos as seguintes hipóteses: 
(a) Seja $\Theta$ um conjunto aberto do espaço Euclidiano $k$-dimensional. Assim, o valor verdadeiro $\theta_{0}$ é um ponto interior de $\Theta$.

(b) $Q_{n}(Z, \theta)$ é uma função mensurável de $Z$ para todo $\theta \in \Theta$, e $\partial Q_{n} / \partial \theta$ existe e é contínua em uma vizinhança aberta $N_{1}\left(\theta_{0}\right)$. Note que isto implica que $Q_{n}$ é contínua para $\theta \in N_{1}$.

(c) Existe uma vizinhança aberta $N_{2}\left(\theta_{0}\right)$ de $\theta_{0}$ de maneira que $n^{-1} Q_{n}(z, \theta)$ converge uniformente em probabilidade em $\theta$ em $N_{2}\left(\theta_{0}\right)$ para uma função não estocástica $Q(\theta)$, e $Q(\theta)$ atinge um mínimo local estrito em $\theta_{0}$.

Seja $\Theta_{n}$ o conjunto das raízes da equação

$$
\frac{\partial Q_{n}}{\partial \theta}=0
$$

correspondentes aos mínimos locais. Se este conjunto for vazio, faça $\Theta_{n}=\{0\}$. Então, para qualquer $\epsilon>0$,

$$
\lim _{n \rightarrow \infty} \operatorname{Pr}\left[\inf _{\theta \in \Theta_{n}}\left(\theta-\theta_{0}\right)^{\prime}\left(\theta-\theta_{0}\right)>\epsilon\right]=0 .
$$

Prova. Ver Amemiya (1985), teorema 4.1.2, pg. 110.

A conclusão do teorema anterior simplesmente diz que um dos mínimos locais (raízes da eq. A.1) é consistente.

\section{Teorema A.3 (Normalidade assintótica, Amemiva (1985), Teor. 4.1.3, pg. 111)}

Façamos as seguintes hipóteses adicionais às estabelecidas no teorema anterior:

(a) $\partial^{2} Q_{n} / \partial \theta \partial \theta^{\prime}$ existe e é contínua em uma vizinhança aberta e convexa de $\theta_{0}$.

(b) $n^{-1}\left(\partial^{2} Q_{n} / \partial \theta \partial \theta^{\prime}\right)_{\theta_{n}^{*}}$ converge em probabilidade para uma matriz não singular $B\left(\theta_{0}\right)=$ $\lim _{n \rightarrow \infty} E\left[n^{-1}\left(\partial^{2} Q_{n} / \partial \theta \partial \theta^{\prime}\right)_{\theta_{0}}\right]$ para toda seqüência $\theta_{n}^{*}$ tal que $\theta_{n}^{*} \stackrel{p}{\longrightarrow} \theta_{0}$.

(c) $n^{-1 / 2}\left(\partial Q_{n} / \partial \theta\right)_{\theta_{0}} \quad \stackrel{d}{\longrightarrow} \quad N\left(0, A\left(\theta_{0}\right)\right), \quad$ onde $\quad A\left(\theta_{0}\right) \quad=$ $\lim _{n \rightarrow \infty} E\left[n^{-1}\left(\partial Q_{n} / \partial \theta\right)_{\theta_{0}} \cdot\left(\partial Q_{n} / \partial \theta^{\prime}\right)_{\theta_{0}}\right]$.

Seja $\left\{\widehat{\theta}_{n}\right\}$ a seqüência obtida pela escolha de um elemento de $\Theta_{n}$ definido no teorema anterior, de tal forma que $\widehat{\theta}_{n} \stackrel{p}{\longrightarrow} \theta_{0}$ (chamamos $\widehat{\theta}_{n}$ de raiz consistente).

Então,

$$
\sqrt{n}\left(\widehat{\theta}_{n}-\theta_{0}\right) \stackrel{d}{\longrightarrow} N\left(0, B\left(\theta_{0}\right)^{-1} A\left(\theta_{0}\right) B\left(\theta_{0}\right)^{-1}\right)
$$

Prova. Vamos apresentar a demonstração apresentada por Amemiya (1985), teorema 4.1.3, pg.111, pois vamos utilizá-la na demonstração das propriedades assintóticas do estimador 
CECF. O estimador de extremo $\widehat{\theta}_{n}$ é obtido resolvendo-se o conjunto de equações (A.1). Assim, expandindo $\partial Q_{n} / \partial \theta$ em uma série de Taylor obtemos

$$
\left.\frac{\partial Q_{n}}{\partial \theta}\right|_{\widehat{\theta}_{n}}=\left.\frac{\partial Q_{n}}{\partial \theta}\right|_{\theta_{0}}+\left.\frac{\partial^{2} Q_{n}}{\partial \theta \partial \theta^{\prime}}\right|_{\theta^{*}}\left(\widehat{\theta}_{n}-\theta_{0}\right)
$$

onde $\theta^{*}$ fica entre $\widehat{\theta}_{n}$ e $\theta_{0}$. Como $\left.\frac{\partial Q_{n}}{\partial \theta}\right|_{\widehat{\theta}_{n}}=0$ pela definição de $\widehat{\theta}_{n}$, obtemos

$$
\sqrt{n}\left(\widehat{\theta}_{n}-\theta_{0}\right)=-\left[\left.\frac{1}{n} \frac{\partial^{2} Q_{n}}{\partial \theta \partial \theta^{\prime}}\right|_{\theta^{*}}\right]^{-1}\left(\left.\frac{1}{\sqrt{n}} \frac{\partial Q_{n}}{\partial \theta}\right|_{\theta_{0}}\right) .
$$

Como $\theta^{*} \stackrel{p}{\rightarrow} \theta_{0}$, então pela hipótese (b) do enunciado,

$$
\left.\frac{1}{n} \frac{\partial^{2} Q_{n}}{\partial \theta \partial \theta^{\prime}}\right|_{\theta^{*}} \stackrel{p}{\rightarrow} B\left(\theta_{0}\right)
$$

Finalmente da hipótese (c) do enunciado, e aplicando o teorema de Slutsky em (A.4), obtemos

$$
\sqrt{n}\left(\widehat{\theta}_{n}-\theta_{0}\right) \stackrel{d}{\longrightarrow} N\left(0, B\left(\theta_{0}\right)^{-1} A\left(\theta_{0}\right) B\left(\theta_{0}\right)^{-1}\right) .
$$

Note que o resultado do teorema anterior fica inalterado se alterarmos as hipóteses (b) e (c) do enunciado para

(a) $\partial^{2} Q_{n} / \partial \theta \partial \theta^{\prime}$ existe e é contínua em uma vizinhança aberta e convexa de $\theta_{0}$.

(b) $\left(\partial^{2} Q_{n} / \partial \theta \partial \theta^{\prime}\right)_{\theta_{n}^{*}}$ converge em probabilidade para uma matriz não singular $B\left(\theta_{0}\right)=$ $\lim _{n \rightarrow \infty} E\left[\left(\partial^{2} Q_{n} / \partial \theta \partial \theta^{\prime}\right)_{\theta_{0}}\right]$ para toda seqüência $\theta_{n}^{*}$ tal que $\theta_{n}^{*} \stackrel{p}{\longrightarrow} \theta_{0}$.

(c) $n^{1 / 2}\left(\partial Q_{n} / \partial \theta\right)_{\theta_{0}} \stackrel{d}{\longrightarrow} N\left(0, A\left(\theta_{0}\right)\right)$, onde $A\left(\theta_{0}\right)=\lim _{n \rightarrow \infty} E\left[n\left(\partial Q_{n} / \partial \theta\right)_{\theta_{0}} \cdot\left(\partial Q_{n} / \partial \theta^{\prime}\right)_{\theta_{0}}\right]$.

Para verificar que o resultado (A.2) permanence válido basta observar que podemos alterar a expansão em série de Taylor (A.4) para

$$
\sqrt{n}\left(\widehat{\theta}_{n}-\theta_{0}\right)=-\left[\left.\frac{\partial^{2} Q_{n}}{\partial \theta \partial \theta^{\prime}}\right|_{\theta^{*}}\right]^{-1}\left(\left.\sqrt{n} \frac{\partial Q_{n}}{\partial \theta}\right|_{\theta_{0}}\right) .
$$

Como $\theta^{*} \stackrel{p}{\rightarrow} \theta_{0}$, então pela hipótese (b),

$$
\left.\frac{\partial^{2} Q_{n}}{\partial \theta \partial \theta^{\prime}}\right|_{\theta^{*}} \stackrel{p}{\rightarrow} B\left(\theta_{0}\right)
$$

Finalmente da hipótese (c), e aplicando o teorema de Slutsky em (A.5), obtemos o resultado (A.4). 


\section{A.2 O método generalizado dos momentos (GMM)}

O método dos momentos foi introduzido por Karl Pearson em 1895 para obter estimadores dos parâmetros, e consiste essencialmente em resolver o sistema de equações obtido ao igualar-se um conjunto de momentos amostrais aos respectivos momentos populacionais (Stuart e Ord (1994)). Para que o sistema tenha solução única utilizamos um número de condições de momentos igual ao número de parâmetros que queremos estimar, ou seja, resolvemos um sistema determinado. Quando possuimos um número maior de condições de momentos do que o número de parâmetros que queremos estimar (ou seja,quando o sistema é sobredeterminado) devemos empregar o método generalizado dos momentos (GMM), cujo objetivo é o de minimizar uma distância entre os momentos amostrais e os momentos teóricos.

Seguindo o texto de Yu (2004) vamos supor que possuamos as seguintes $q$ condições de momentos:

$$
E\left[f\left(X_{j} ; \theta_{0}\right)\right]=0,
$$

onde $f: \mathbb{R} \times \mathbb{R}^{k} \rightarrow \mathbb{R}^{q}$. Assumamos adicionalmente que possamos invocar a lei forte dos grandes números de maneira a obtermos o seguinte resultado para os momentos amostrais:

$$
\frac{1}{n} \sum_{j=1}^{n} f\left(X_{j} ; \theta\right) \stackrel{\text { a.s. }}{\longrightarrow} E\left[f\left(X_{j} ; \theta\right)\right] .
$$

A idéia básica da estimação GMM é minimizar a distância entre os momentos amostrais e os momentos populacionais, isto é,

$$
\min _{\theta} \frac{1}{n} \sum_{j=1}^{n} f\left(X_{j} ; \theta\right)^{\prime} W_{n} \frac{1}{n} \sum_{j=1}^{n} f\left(X_{j} ; \theta\right),
$$

onde $W_{n}$ é uma matriz de pesos positiva definida que converge quase certamente para uma matriz positiva definida $W_{0}$. Sob algumas condições de regularidade, o estimador GMM é consistente e distribuído assintoticamente normal para matrizes de peso arbitrárias. Quando o sistema é identificado $(k=q)$, o estimador GMM não depende da escolha de $W_{n}$ e basicamente resolve as equações de estimação do método dos momentos: $1 / n \sum_{j=1}^{n} f\left(X_{j} ; \theta\right)=0$. Quando o sistema é sobre-identificado $(k<q)$, Hansen (1982) mostra que se $W_{0}=\Sigma^{-1}$, o estimador GMM é assintoticamente eficiente no sentido que a matriz de covariância do estimador GMM é minimizada, sendo $\Sigma$ a matriz de covariância assintótica de $f\left(X_{j} ; \theta\right)$.

Agora vamos rever as hipóteses e o resultado de convergência assintótica do estimador GMM como obtido por Hansen (1982). Inicialmente vamos usar as seguintes variáveis:

$$
\begin{gathered}
f_{n}(\omega, \beta)=f\left[x_{n}(\omega, \beta)\right], \\
g_{N}(\omega, \beta)=\frac{1}{N} \sum_{n=1}^{N} f_{n}(\omega, \beta), \\
h_{N}(\omega, \beta)=a_{N}(\omega) g_{N}(\omega, \beta)
\end{gathered}
$$




$$
\begin{gathered}
B_{N}(\omega)=\left\{\beta \in S:\left|h_{N}(\omega, \beta)\right|^{2}=\inf _{\beta \in S}\left|h_{N}(\omega, \beta)\right|^{2}\right\}, \\
w_{n}=f\left(x_{n}, \beta_{0}\right),-\infty<n<+\infty, \\
v_{j}=E\left[w_{0} \mid w_{-j}, w_{-j-1}, \cdots\right]-E\left[w_{0} \mid w_{-j-1}, w_{-j-2}, \cdots\right], j \geq 0 .
\end{gathered}
$$

Vamos agora replicar as hipóteses utilizadas por Hansen (1982), utilizando a notação $d_{0}=E\left[\partial f / \partial \beta\left(x_{1}, \beta_{0}\right)\right]:$

(a) $\left\{x_{n}:-\infty<n<+\infty\right\}$ é estacionário e ergódico.

(b) $S$ é um subconjunto aberto de $\mathbb{R}^{q}$ que contém $\beta_{0}$.

(c) $f(., \beta)$ e $\partial f / \partial \beta(., \beta)$ são Borel-mensuráveis para cada $\beta \in S$ e $\partial f / \partial \beta(x, \beta)$ é contínuo em $S$ para cada $x \in \mathbb{R}^{p}$.

(d) $\partial f_{1} / \partial \beta$ tem primeiro momento contínuo em $\beta_{0}$, e $E\left[\partial f / \partial \beta\left(x_{1}, \beta_{0}\right)\right]$ existe, é finito, e tem posto completo.

(e) $E\left[w_{0} w_{0}^{\prime}\right]$ existe e é finita, $E\left[w_{0} \mid w_{-j}, w_{-j-1}, \cdots\right]$ converge em média quadrática para zero, e $\sum_{j=0}^{\infty} E\left[v_{j}^{\prime} v_{j}\right]^{1 / 2}$ é finito.

(f) $\left\{a_{N}^{*}: N \geq 1\right\}$ converge em probabilidade para uma matriz de constantes $a_{0}^{*}$ que tem posto completo.

O estimador GMM $\left\{b_{N}^{*}: N \geq 1\right\}$ deve satisfazer assintoticamente o seguinte conjunto de equações:

$$
a_{0}^{*} E f\left(x_{n}, \beta\right)=0
$$

Definição A.1 (Estimador GMM, def. 3.1 Hansen (1982)) O estimador GMM $\left\{b_{N}^{*}: N \geq 1\right\}$ é uma seqüência de vetores aleatórios que converge em probabilidade para $\beta_{0}$ para o qual $\left\{\sqrt{N} a_{N}^{*} g_{N}\left(b_{N}^{*}\right): N \geq 1\right\}$ converge em probabilidade para zero.

Lema A.1 (Lema 3.2, Hansen (1982)) Suponha que as hipóteses (a) a (d) estejam satisfeitas. Se (i) $\left\{b_{N}: N \geq 1\right\}$ converge em probabilidade para $\beta_{0}$; (ii) $\left\{a_{N}: N \geq 1\right\}$ converge em probabilidade para $a_{0}$; então $\left\{\left(\partial g_{N} / \partial \beta\right)\left(b_{N}\right): N \geq 1\right\}$ converge em probabilidade para $d_{0}$ $e\left\{a_{N}^{*}: N \geq 1\right\}$ definido abaixo converge em probabilidade para $a_{0}^{*}=d_{0}^{\prime} a_{0}^{\prime} a_{0}$

$$
a_{N}^{*}=\frac{\partial g_{N}}{\partial \beta}\left(b_{N}\right)^{\prime} a_{N}^{\prime} a_{N}
$$


Teorema A.4 (Teor. 3.1, Hansen (1982)) Suponha que as hipóteses (a) a (f) estejam satisfeitas. Então $\left\{\sqrt{N}\left(b_{N}^{*}-\beta_{0}\right): N \geq 1\right\}$ converge em distribuição para um vetor aleatório normalmente distribuído com média zero e matriz de covariância $\left(a_{0}^{*} d_{0}\right)^{-1} a_{0}^{*} S_{w} a_{0}^{* \prime}\left(a_{0}^{*} d_{0}\right)^{-1 \prime}$, sendo que pelas hipóteses (a) e (e)

$$
S_{w}=\sum_{j=-\infty}^{+\infty} E\left[w_{0} w_{-j}^{\prime}\right]
$$

é bem definido e finito.

Prova. A prova deste teorema encontra-se em Hansen (1982), pg. 1051 (apêndice).

Podemos observar pelo resultado acima que a matriz de covariância do estimador GMM é geralmente diferente do inverso da matriz de informação de Fisher. Assim, geralmente o estimador GMM não possui a mesma eficiência assintótica que o estimador de máxima verossimilhança. 


\section{A.3 Um Teorema Central Limite para processos estacionários}

O objetivo desta seção é apresentar um teorema central limite para seqüências dependentes, utilizado na demonstração da convergência do estimador CECF. Inicialmente vamos definir algumas condições de dependência da seqüência, conhecidas como condições mixing. Para isso vamos seguir o texto de White (2000), embora outra referência interessante sobre o assunto seja Davidson (1994), cap. 13 e 14.

Definição A.2 A $\sigma$-álgebra de Borel gerada por $\left\{X_{t}, t=n, \cdots, n+m\right\}$, denotada por $\mathcal{B}_{n}^{n+m}=\sigma\left(X_{n}, \cdots, X_{n+m}\right)$, é a menor $\sigma$-álgebra de $\Omega$ que inclui

(a) todos os conjuntos da forma $\times_{i=1}^{n-1} \mathbb{R}^{q} \times_{i=n}^{n+m} B_{i} \times{ }_{i=n+m+1}^{\infty} \mathbb{R}^{q}$, onde cada $B_{i} \in \mathcal{B}^{q}$;

(b) o complemento $A^{c}$ de qualquer conjunto $A$ em $\mathcal{B}_{n}^{n+m}$;

(c) a união $\bigcup_{i=1}^{\infty} A_{i}$ de qualquer seqüência $\left\{A_{i}\right\}$ em $\mathcal{B}_{n}^{n+m}$.

Definição A.3 Seja $\mathcal{B}_{-\infty}^{n} \equiv \sigma\left(\cdots, X_{n}\right)$ a menor coleção de subconjuntos de $\Omega$ que contenha a união das $\sigma$-álgebras $\mathcal{B}_{a}^{n}$ quando $a \rightarrow-\infty$; seja $\mathcal{B}_{n+m}^{\infty} \equiv \sigma\left(X_{n+m}, \cdots\right)$ a menor coleção de subconjuntos de $\Omega$ que contenha a união das $\sigma$-álgebras $\mathcal{B}_{n+m}^{a}$ quando $a \rightarrow \infty$.

Intuitivamente podemos pensar em $\mathcal{B}_{-\infty}^{n}$ como toda a informação contida no passado da seqüência $\left\{X_{t}\right\}$, até o instante $n$, enquanto que $\mathcal{B}_{n+m}^{\infty}$ representa a a informação contida no futuro da seqüência $\left\{X_{t}\right\}$ a partir de $n+m$.

Definição A.4 Sejam $\mathcal{G}$ e $\mathcal{H}$ o-álgebras e defina

$$
\begin{aligned}
\phi(\mathcal{G}, \mathcal{H}) & \equiv \sup _{G \in \mathcal{G}, H \in \mathcal{H}, P(G)>0}|P(H \mid G)-P(H)| \\
\alpha(\mathcal{G}, \mathcal{H}) & \equiv \sup _{G \in \mathcal{G}, H \in \mathcal{H}}|P(G \cap H)-P(G) P(H)|
\end{aligned}
$$

Note que os eventos em $\mathcal{G}$ e $\mathcal{H}$ são independentes se e somente se $\phi(\mathcal{G}, \mathcal{H})$ e $\alpha(\mathcal{G}, \mathcal{H})$ são iguais a zero. Assim, a função $\alpha$ fornece uma medida absoluta, enquanto $\phi$ fornece uma medida relativa, da dependência entre eventos em $\mathcal{G}$ e $\mathcal{H}$.

Definição A.5 Para uma seqüência de vetores aleatórios $\left\{X_{t}\right\}$ definimos os coeficientes mixing como

$$
\begin{aligned}
\phi(m) & \equiv \sup _{n} \phi\left(\mathcal{B}_{-\infty}^{n}, \mathcal{B}_{n+m}^{\infty}\right) \\
\alpha(m) & \equiv \sup _{n} \alpha\left(\mathcal{B}_{-\infty}^{n}, \mathcal{B}_{n+m}^{\infty}\right) .
\end{aligned}
$$


Se $\phi(m) \rightarrow 0$ quando $m \rightarrow \infty$, então a seqüência $\left\{X_{t}\right\}$ é dita $\phi$-mixing, ou mixing uniforme. Se $\alpha(m) \rightarrow 0$ quando $m \rightarrow \infty$, então a seqüência $\left\{X_{t}\right\}$ é dita $\alpha$-mixing, ou mixing forte.

O conceito de mixing forte $(\alpha)$ é mais fraco do que o conceito de mixing uniforme $(\phi)$ no sentido de que $\phi$-mixing implica em $\alpha$-mixing, uma vez que

$$
|P(G \cap H)-P(G) P(H)| \leq|P(H \mid G)-P(H)| \leq \phi(G, H)
$$

para todo $G \in \mathcal{G}$ e $H \in \mathcal{H}$, e portanto $\alpha(\mathcal{G}, \mathcal{H}) \leq \phi(\mathcal{G}, \mathcal{H})$.

Vamos a seguir apresentar um teorema central limite para seqüências com mixing forte.

Teorema A.5 (Teorema 18.5.4, Ibragimov e Linnik, 1971) Seja a seqüência estacionária mixing forte $X_{j}$, com

$$
\sum_{n=1}^{\infty} \alpha(n)<\infty
$$

$X_{j}$ limitada, $\operatorname{Pr}\left(\left|X_{j}\right|<c_{0}\right)=1$. Então

$$
\sigma^{2}=E\left(X_{0}^{2}\right)+2 \sum_{j=1}^{\infty} E\left(X_{0} X_{j}\right)<\infty
$$

$e$, se $\sigma \neq 0$,

$$
\lim _{n \rightarrow \infty} P\left\{\sigma^{-1} n^{-1 / 2} \sum_{j=1}^{n} X_{j}<z\right\}=\Phi(z) .
$$

Prova. Ver Ibragimov e Linnik (1971), teorema 18.5.4, pg. 347. 



\section{Apêndice B}

\section{Covariância do processo $Y_{n}$}

É comum vermos em artigos a frase "é fácil ver que...", ou então a frase "depois de uma álgebra simples, mas tediosa...", com as quais os autores justificam o "salto"de passagens da demonstração para ir direto ao ponto desejado. Quando nos deparamos com este tipo de frase, imaginamos imediatamente que vamos demorar várias horas e muitas páginas para chegar ao resultado final...

Assim, com o espírito de procurar não pular passagens, mas sim apresentar os detalhes da tediosa álgebra, vamos apresentar neste apêndice as demonstrações das matrizes de covariância $\Omega_{n}$ do processo da função característica empírica, tanto para o caso de variáveis aleatórias i.i.d., quanto para o caso estacionário.

\section{B.1 Caso i.i.d.}

Nesta seção vamos obter a estrutura de covariância do processo $Y_{n}(r)=\sqrt{n}\left(\varphi_{n}(r)-\varphi(r)\right)$ indicada no teorema 2.3, que se aplica ao caso i.i.d.. Para isso, notemos que:

$$
\varphi_{n}(r)=\frac{1}{n} \sum_{j=1}^{n} e^{i r^{\prime} X_{j}}=\frac{1}{n} \sum_{j=1}^{n}\left[\cos \left(r^{\prime} X_{j}\right)+i \sin \left(r^{\prime} X_{j}\right)\right] .
$$

Chamemos

$$
\begin{aligned}
& u_{n}(r)=\operatorname{Re} \varphi_{n}(r)=\frac{1}{n} \sum_{j=1}^{n} \cos \left(r^{\prime} X_{j}\right) \\
& v_{n}(r)=\operatorname{Im} \varphi_{n}(r)=\frac{1}{n} \sum_{j=1}^{n} \sin \left(r^{\prime} X_{j}\right) \\
& u(r)=\operatorname{Re} \varphi(r)=E\left[\cos \left(r^{\prime} X_{j}\right)\right] \\
& v(r)=\operatorname{Im} \varphi(r)=E\left[\sin \left(r^{\prime} X_{j}\right)\right]
\end{aligned}
$$


Também é conveniente recordarmos as seguintes relações trigonométricas:

$$
\begin{aligned}
\sin A \sin B & =\frac{1}{2}\{\cos (A-B)-\cos (A+B)\} \\
\cos A \cos B & =\frac{1}{2}\{\cos (A-B)+\cos (A+B)\} \\
\sin A \cos B & =\frac{1}{2}\{\sin (A-B)+\sin (A+B)\}
\end{aligned}
$$

Podemos calcular a covariância $\operatorname{cov}\left[Y_{n}(r), Y_{n}(s)\right]$ por:

$$
\begin{aligned}
\operatorname{cov}\left[Y_{n}(r), Y_{n}(s)\right] & =E\left[Y_{n}(r) . \overline{Y_{n}(s)}\right] \\
& =E\left\{\sqrt{n}\left(\varphi_{n}(r)-\varphi(r)\right) \overline{\sqrt{n}\left(\varphi_{n}(s)-\varphi(s)\right)}\right\} \\
& =n E\left\{\left(\varphi_{n}(r)-\varphi(r)\right)\left(\overline{\varphi_{n}(s)}-\overline{\varphi(s)}\right)\right\} \\
& =n E\left\{\varphi_{n}(r) \overline{\varphi_{n}(s)}-\varphi_{n}(r) \overline{\varphi(s)}-\varphi(r) \overline{\varphi_{n}(s)}+\varphi(r) \overline{\varphi(s)}\right\} \\
& =n\left\{E\left[\varphi_{n}(r) \overline{\varphi_{n}(s)}\right]-\varphi(r) \overline{\varphi(s)}-\varphi(r) \overline{\varphi(s)}+\varphi(r) \overline{\varphi(s)}\right\} \\
& =n\left\{E\left[\varphi_{n}(r) \overline{\varphi_{n}(s)}\right]-\varphi(r) \overline{\varphi(s)}\right\} \\
& =n\left\{E\left[\varphi_{n}(r) \varphi_{n}(-s)\right]-\varphi(r) \varphi(-s)\right\} \\
& =n E\left[\varphi_{n}(r) \varphi_{n}(-s)\right]-n \varphi(r) \varphi(-s)
\end{aligned}
$$

onde utilizamos a propriedade $\varphi(r)=\overline{\varphi(-r)}$. Agora, como por hipótese $X_{j}$ é independente de $X_{k}$ para $j \neq k$, temos

$$
\begin{aligned}
E\left[\varphi_{n}(r) \varphi_{n}(-s)\right] & =E\left\{\frac{1}{n} \sum_{j=1}^{n} e^{i r^{\prime} X_{j}} \cdot \frac{1}{n} \sum_{k=1}^{n} e^{-i s^{\prime} X_{k}}\right\} \\
& =\frac{1}{n^{2}} E\left\{\sum_{j=1}^{n} \sum_{k=1}^{n} e^{i r^{\prime} X_{j}} e^{-i s^{\prime} X_{k}}\right\} \\
& =\frac{1}{n^{2}} E\left\{\sum_{j=1}^{n} e^{i(r-s)^{\prime} X_{j}}+\sum_{j=1}^{n} \sum_{\substack{k=1 \\
j \neq k}}^{n} e^{i r^{\prime} X_{j}-i s^{\prime} X_{k}}\right\} \\
& =\frac{1}{n^{2}}\left\{\sum_{j=1}^{n} E\left[e^{i(r-s)^{\prime} X_{j}}\right]+\sum_{\substack{j=1 \\
j=1}}^{n} \sum_{\substack{k=1 \\
j \neq k}}^{n} E\left[e^{i r^{\prime} X_{j}}\right] E\left[e^{\left.-i s^{\prime} X_{k}\right]}\right\}\right. \\
& =\frac{1}{n^{2}}\{n \varphi(r-s)+n(n-1) \varphi(r) \varphi(-s)\} \\
& =\frac{1}{n}\{\varphi(r-s)+(n-1) \varphi(r) \varphi(-s)\}
\end{aligned}
$$


Substituindo (B.10) em (B.9) obtemos

$$
\begin{aligned}
\operatorname{cov}\left[Y_{n}(r), Y_{n}(s)\right] & =n E\left[\varphi_{n}(r) \varphi_{n}(-s)\right]-n \varphi(r) \varphi(-s) \\
& =\varphi(r-s)+(n-1) \varphi(r) \varphi(-s)-n \varphi(r) \varphi(-s) \\
& =\varphi(r-s)-\varphi(r) \varphi(-s)
\end{aligned}
$$

Vamos agora obter as covariâncias entre as partes reais e imaginárias de $Y_{n}(r)$ e $Y_{n}(s)$ :

$$
\begin{aligned}
& \operatorname{cov}\left[\operatorname{Re} Y_{n}(r), \operatorname{Re} Y_{n}(s)\right]=E\left[\operatorname{Re} Y_{n}(r) \cdot \operatorname{Re} Y_{n}(s)\right]= \\
= & E\left[\operatorname{Re} Y_{n}(r) \cdot \operatorname{Re} Y_{n}(s)\right] \\
= & E\left\{\sqrt{n} \operatorname{Re}\left(\varphi_{n}(r)-\varphi(r)\right) \sqrt{n} \operatorname{Re}\left(\varphi_{n}(s)-\varphi(s)\right)\right\} \\
= & n E\left\{\operatorname{Re} \varphi_{n}(r) \operatorname{Re} \varphi_{n}(s)-\operatorname{Re} \varphi_{n}(r) \operatorname{Re} \varphi(s)-\operatorname{Re} \varphi(r) \operatorname{Re} \varphi_{n}(s)+\operatorname{Re} \varphi(r) \operatorname{Re} \varphi(s)\right\} \\
= & n E\left\{\operatorname{Re} \varphi_{n}(r) \operatorname{Re} \varphi_{n}(s)\right\}-n \operatorname{Re} \varphi(r) \operatorname{Re} \varphi(s) \\
= & n E\left\{u_{n}(r) u_{n}(s)\right\}-n u(r) u(s) \\
= & n E\left\{\frac{1}{n} \sum_{j=1}^{n} \cos \left(r^{\prime} X_{j}\right) \frac{1}{n} \sum_{k=1}^{n} \cos \left(s^{\prime} X_{k}\right)\right\}-n u(r) u(s) \\
= & \frac{1}{n} E\left\{\sum_{j=1}^{n} \cos \left(r^{\prime} X_{j}\right) \cos \left(s^{\prime} X_{j}\right)+\sum_{j=1}^{n} \sum_{k=1}^{n} \cos \left(r^{\prime} X_{j}\right) \cos \left(s^{\prime} X_{k}\right)\right\}-n u(r) u(s) \\
= & \frac{1}{n} \sum_{j=1}^{n} E\left\{\cos \left(r^{\prime} X_{j}\right) \cos \left(s^{\prime} X_{j}\right)\right\}+ \\
& +\frac{1}{n} \sum_{j=1}^{n} \sum_{k=1}^{n} E\left\{\cos \left(r^{\prime} X_{j}\right) \cos \left(s^{\prime} X_{k}\right)\right\}-n u(r) u(s) \\
& +\frac{1}{n} \sum_{j=1}^{n} E\left\{\cos \left(r^{\prime} X_{1}\right) \cos \left(s^{\prime} X_{1}\right)\right\}+ \\
& =1 n-k) E\left\{\cos \left(r^{\prime} X_{1}\right) \cos \left(s^{\prime} X_{1+k}\right)\right\}-n u(r) u(s)
\end{aligned}
$$


$=E\left\{\cos \left(r^{\prime} X_{1}\right) \cos \left(s^{\prime} X_{1}\right)\right\}+\frac{2}{n} \frac{n(n-1)}{2} E\left\{\cos \left(r^{\prime} X_{1}\right) \cos \left(s^{\prime} X_{2}\right)\right\}-n u(r) u(s)$

$=E\left\{\cos \left(r^{\prime} X_{1}\right) \cos \left(s^{\prime} X_{1}\right)\right\}+(n-1) E\left\{\cos \left(r^{\prime} X_{1}\right)\right\} E\left\{\cos \left(s^{\prime} X_{2}\right)\right\}-n u(r) u(s)$

$=E\left\{\frac{1}{2}\left[\cos \left((r-s)^{\prime} X_{1}\right)+\cos \left((r+s)^{\prime} X_{1}\right)\right]\right\}+$ $+(n-1) E\left\{\cos \left(r^{\prime} X_{1}\right)\right\} E\left\{\cos \left(s^{\prime} X_{2}\right)\right\}-n u(r) u(s)$

$=\frac{1}{2}\{u(r-s)+u(r+s)\}+(n-1) u(r) u(s)-n u(r) u(s)$

$=\frac{1}{2}\{u(r-s)+u(r+s)\}-u(r) u(s)$

$=\frac{1}{2}\{\operatorname{Re} \varphi(r+s)+\operatorname{Re} \varphi(r-s)\}-\operatorname{Re} \varphi(r) \operatorname{Re} \varphi(s)$

Analogamente obtemos

$$
\begin{aligned}
\operatorname{cov}\left[\operatorname{Re} Y_{n}(r), \operatorname{Im} Y_{n}(s)\right] & =E\left[\operatorname{Re} Y_{n}(r) \cdot \operatorname{Im} Y_{n}(s)\right] \\
& =n E\left\{u_{n}(r) v_{n}(s)\right\}-n u(r) v(s)= \\
& =\frac{1}{2}\{v(s-r)+v(r+s)\}+(n-1) u(r) v(s)-n u(r) v(s) \\
& =\frac{1}{2}\{-v(r-s)+v(r+s)\}-u(r) v(s) \\
& =\frac{1}{2}\{\operatorname{Im} \varphi(r+s)-\operatorname{Im} \varphi(r-s)\}-\operatorname{Re} \varphi(r) \operatorname{Im} \varphi(s)
\end{aligned}
$$

$$
\begin{aligned}
\operatorname{cov}\left[\operatorname{Im} Y_{n}(r), \operatorname{Re} Y_{n}(s)\right] & =E\left[\operatorname{Im} Y_{n}(r) \cdot \operatorname{Re} Y_{n}(s)\right] \\
& =n E\left\{v_{n}(r) u_{n}(s)\right\}-n v(r) u(s) \\
& =\frac{1}{2}\{v(r-s)+v(r+s)\}+(n-1) v(r) u(s)-n v(r) u(s) \\
& =\frac{1}{2}\{v(r-s)+v(r+s)\}-v(r) u(s) \\
& =\frac{1}{2}\{\operatorname{Im} \varphi(r+s)+\operatorname{Im} \varphi(r-s)\}-\operatorname{Im} \varphi(r) \operatorname{Re} \varphi(s)
\end{aligned}
$$

$$
\begin{aligned}
\operatorname{cov}\left[\operatorname{Im} Y_{n}(r), \operatorname{Im} Y_{n}(s)\right] & =E\left[\operatorname{Im} Y_{n}(r) \cdot \operatorname{Im} Y_{n}(s)\right] \\
& =n E\left\{v_{n}(r) v_{n}(s)\right\}-n v(r) v(s) \\
& =\frac{1}{2}\{u(r-s)-u(r+s)\}+(n-1) v(r) v(s)-n v(r) v(s) \\
& =\frac{1}{2}\{u(r-s)-u(r+s)\}-v(r) v(s) \\
& =\frac{1}{2}\{-\operatorname{Re} \varphi(r+s)+\operatorname{Re} \varphi(r-s)\}-\operatorname{Im} \varphi(r) \operatorname{Im} \varphi(s)
\end{aligned}
$$


Resumindo:

$$
\begin{aligned}
\operatorname{cov}\left[\operatorname{Re} Y_{n}(r), \operatorname{Re} Y_{n}(s)\right] & =\frac{1}{2}\{\operatorname{Re} \varphi(r+s)+\operatorname{Re} \varphi(r-s)\}-\operatorname{Re} \varphi(r) \operatorname{Re} \varphi(s) \\
\operatorname{cov}\left[\operatorname{Re} Y_{n}(r), \operatorname{Im} Y_{n}(s)\right] & =\frac{1}{2}\{\operatorname{Im} \varphi(r+s)-\operatorname{Im} \varphi(r-s)\}-\operatorname{Re} \varphi(r) \operatorname{Im} \varphi(s) \\
\operatorname{cov}\left[\operatorname{Im} Y_{n}(r), \operatorname{Re} Y_{n}(s)\right] & =\frac{1}{2}\{\operatorname{Im} \varphi(r+s)+\operatorname{Im} \varphi(r-s)\}-\operatorname{Im} \varphi(r) \operatorname{Re} \varphi(s) \\
\operatorname{cov}\left[\operatorname{Im} Y_{n}(r), \operatorname{Im} Y_{n}(s)\right] & =\frac{1}{2}\{-\operatorname{Re} \varphi(r+s)+\operatorname{Re} \varphi(r-s)\}-\operatorname{Im} \varphi(r) \operatorname{Im} \varphi(s)
\end{aligned}
$$

Como $Y_{n}(r)=\sqrt{n}\left(\varphi_{n}(r)-\varphi(r)\right)$ podemos escrever

$$
\operatorname{cov}\left[\left(\begin{array}{c}
\operatorname{Re} \varphi_{n}(r) \\
\operatorname{Im} \varphi_{n}(r)
\end{array}\right),\left(\begin{array}{c}
\operatorname{Re} \varphi_{n}(s) \\
\operatorname{Im} \varphi_{n}(s)
\end{array}\right)\right]=\frac{1}{n} \Omega(r, s)
$$

onde $\Omega(r, s)=\left(\begin{array}{cc}\Omega_{R R}(r, s) & \Omega_{R I}(r, s) \\ \Omega_{I R}(r, s) & \Omega_{I I}(r, s)\end{array}\right)$, e os elementos da partição são dados pelas equações (B.16) a (B.19):

$$
\begin{aligned}
\Omega_{R R}(r, s) & =\frac{1}{2}\{\operatorname{Re} \varphi(r+s)+\operatorname{Re} \varphi(r-s)\}-\operatorname{Re} \varphi(r) \operatorname{Re} \varphi(s) \\
\Omega_{R I}(r, s) & =\frac{1}{2}\{\operatorname{Im} \varphi(r+s)-\operatorname{Im} \varphi(r-s)\}-\operatorname{Re} \varphi(r) \operatorname{Im} \varphi(s) \\
\Omega_{I R}(r, s) & =\frac{1}{2}\{\operatorname{Im} \varphi(r+s)+\operatorname{Im} \varphi(r-s)\}-\operatorname{Im} \varphi(r) \operatorname{Re} \varphi(s) \\
\Omega_{I I}(r, s) & =\frac{1}{2}\{-\operatorname{Re} \varphi(r+s)+\operatorname{Re} \varphi(r-s)\}-\operatorname{Im} \varphi(r) \operatorname{Im} \varphi(s)
\end{aligned}
$$

As equações (B.21) a (B.24) coincidem com o caso de amostras i.i.d., como obtido, por exemplo, em Heathcote (1977). 


\section{B.2 Caso estacionário (não necessariamente i.i.d.)}

Seja $\left\{Z_{j}\right\}$ um processo estacionário de v.a. não necessariamente independentes e identicamente distribuídas, com função característica empírica $\varphi_{n}(r)$ e função característica $\varphi(r)$. Neste caso a covariância entre $Y_{n}(r)$ e $Y_{n}(s)$ ainda é dada por (B.9),

$$
\operatorname{cov}\left[Y_{n}(r), Y_{n}(s)\right]=n E\left[\varphi_{n}(r) \varphi_{n}(-s)\right]-n \varphi(r) \varphi(-s),
$$

mas já não podemos utilizar (B.11) devido a não independência entre $Z_{j}$ e $Z_{j+k}$. Vamos utilizar então a seguinte função auxiliar

$$
\Lambda_{k}(r, s)=E\left[e^{i r^{\prime} Z_{j}+i s^{\prime} Z_{j+k}}\right] .
$$

Note também que podemos escrever (B.25) como

$$
\begin{aligned}
\Lambda_{k}(r, s)= & E\left[\exp \left(i r^{\prime} Z_{j}+i s^{\prime} Z_{j+k}\right)\right] \\
= & E\left[\cos \left(r^{\prime} Z_{j}+s^{\prime} Z_{j+k}\right)\right]+i E\left[\sin \left(r^{\prime} Z_{j}+s^{\prime} Z_{j+k}\right)\right] \\
= & E\left[\cos \left(r^{\prime} Z_{j}\right) \cos \left(s^{\prime} Z_{j+k}\right)-\sin \left(r^{\prime} Z_{j}\right) \sin \left(s^{\prime} Z_{j+k}\right)\right]+ \\
& +i E\left[\sin \left(r^{\prime} Z_{j}\right) \cos \left(s^{\prime} Z_{j+k}\right)+\cos \left(r^{\prime} Z_{j}\right) \sin \left(s^{\prime} Z_{j+k}\right)\right] .
\end{aligned}
$$

onde utilizamos as relações trigonométricas

$$
\begin{aligned}
& \cos (A+B)=\cos A \cos B-\sin A \sin B \\
& \sin (A+B)=\sin A \cos B+\cos A \sin B .
\end{aligned}
$$

Utilizando (B.1) podemos escrever $E\left[\varphi_{n}(r) \varphi_{n}(-s)\right]$ como

$$
\begin{gathered}
E\left[\varphi_{n}(r) \varphi_{n}(-s)\right]= \\
=E\left\{\frac{1}{n} \sum_{j=1}^{n} e^{i r^{\prime} Z_{j}} \frac{1}{n} \sum_{k=1}^{n} e^{-i s^{\prime} Z_{k}}\right\} \\
=\frac{1}{n^{2}} E\left\{\sum_{j=1}^{n} \sum_{k=1}^{n} e^{i r^{\prime} Z_{j}} e^{-i s^{\prime} Z_{k}}\right\} \\
=\frac{1}{n^{2}} E\left\{\sum_{j=1}^{n} e^{i(r-s)^{\prime} Z_{j}}+\sum_{j=1}^{n} \sum_{\substack{k=1 \\
j \neq k}}^{n} e^{i r^{\prime} Z_{j}-i s^{\prime} Z_{k}}\right\}
\end{gathered}
$$




$$
\begin{aligned}
= & \frac{1}{n^{2}}\left\{\sum_{j=1}^{n-1} E\left[e^{i(r-s)^{\prime} Z_{j}}\right]+\sum_{j=1}^{n} \sum_{\substack{k=1 \\
j \neq k}}^{n} E\left[e^{i r^{\prime} Z_{j}-i s^{\prime} Z_{k}}\right]\right\} \\
= & \frac{1}{n^{2}}\left\{n E\left[e^{i(r-s)^{\prime} Z_{1}}\right]+\sum_{k=1}^{n-1}(n-k) E\left[e^{i r^{\prime} Z_{1}-i s^{\prime} Z_{1+k}}\right]+\right. \\
& \left.+\sum_{k=1}^{n}(n-k) E\left[e^{i r^{\prime} Z_{1+k}-i s^{\prime} Z_{1}}\right]\right\} \\
= & \frac{1}{n}\left\{\varphi(r-s)+\sum_{k=1}^{n-1} \frac{(n-k)}{n} \Lambda_{k}(r,-s)+\sum_{k=1}^{n-1} \frac{(n-k)}{n} \Lambda_{k}(-s, r)\right\}
\end{aligned}
$$

onde usamos a hipótese de estacionaridade de $Z_{j}$ na passagem da $4^{a}$ para a $5^{a}$ linha.

Substituindo (B.27) em (B.9) obtemos

$$
\begin{aligned}
\operatorname{cov}\left[Y_{n}(r), Y_{n}(s)\right] & =\varphi(r-s)+\sum_{k=1}^{n-1} \frac{(n-k)}{n} \Lambda_{k}(r,-s)+\sum_{k=1}^{n-1} \frac{(n-k)}{n} \Lambda_{k}(-s, r)-n \varphi(r) \varphi(-s) \\
& =\varphi(r-s)-n \varphi(r) \varphi(-s)+\sum_{k=1}^{n-1} \frac{(n-k)}{n}\left[\Lambda_{k}(r,-s)+\Lambda_{k}(-s, r)\right] \quad \text { (B.28) }
\end{aligned}
$$

Vamos agora obter as covariâncias entre as partes reais e imaginárias de $Y_{n}(r)$ e $Y_{n}(s)$. A covariância entre as partes reais é dada por: 


$$
\begin{aligned}
& \operatorname{cov}\left[\operatorname{Re} Y_{n}(r), \operatorname{Re} Y_{n}(s)\right]=E\left[\operatorname{Re} Y_{n}(r) \cdot \operatorname{Re} Y_{n}(s)\right]= \\
& =E\left\{\sqrt{n} \operatorname{Re}\left(\varphi_{n}(r)-\varphi(r)\right) \sqrt{n} \operatorname{Re}\left(\varphi_{n}(s)-\varphi(s)\right)\right\} \\
& =n E\left\{\operatorname{Re} \varphi_{n}(r) \operatorname{Re} \varphi_{n}(s)\right\}-n \operatorname{Re} \varphi(r) \operatorname{Re} \varphi(s) \\
& =n E\left\{u_{n}(r) u_{n}(s)\right\}-n u(r) u(s) \\
& =n E\left\{\frac{1}{n} \sum_{j=1}^{n} \cos \left(r^{\prime} X_{j}\right) \frac{1}{n} \sum_{k=1}^{n} \cos \left(s^{\prime} X_{k}\right)\right\}-n u(r) u(s) \\
& =\frac{1}{n} E\left\{\sum_{j=1}^{n} \cos \left(r^{\prime} X_{j}\right) \cos \left(s^{\prime} X_{j}\right)+\sum_{j=1}^{n} \sum_{\substack{k=1 \\
k \neq j}}^{n} \cos \left(r^{\prime} X_{j}\right) \cos \left(s^{\prime} X_{k}\right)\right\}-n u(r) u(s) \\
& =\frac{1}{n} \sum_{j=1}^{n} E\left\{\cos \left(r^{\prime} X_{j}\right) \cos \left(s^{\prime} X_{j}\right)\right\}+ \\
& +\frac{1}{n} \sum_{j=1}^{n} \sum_{\substack{k=1 \\
k \neq j}}^{n} E\left\{\cos \left(r^{\prime} X_{j}\right) \cos \left(s^{\prime} X_{k}\right)\right\}-n u(r) u(s) \\
& =\frac{1}{n} \sum_{j=1}^{n} E\left\{\cos \left(r^{\prime} X_{1}\right) \cos \left(s^{\prime} X_{1}\right)\right\}+\frac{1}{n} \sum_{k=1}^{n-1}(n-k) E\left\{\cos \left(r^{\prime} X_{1}\right) \cos \left(s^{\prime} X_{1+k}\right)\right\}+ \\
& +\frac{1}{n} \sum_{k=1}^{n-1}(n-k) E\left\{\cos \left(r^{\prime} X_{1+k}\right) \cos \left(s^{\prime} X_{1}\right)\right\}-n u(r) u(s) \\
& =E\left\{\cos \left(r^{\prime} X_{1}\right) \cos \left(s^{\prime} X_{1}\right)\right\}+\frac{1}{n} \sum_{k=1}^{n-1}(n-k) E\left\{\cos \left(r^{\prime} X_{1}\right) \cos \left(s^{\prime} X_{1+k}\right)\right\}+ \\
& +\frac{1}{n} \sum_{k=1}^{n-1}(n-k) E\left\{\cos \left(r^{\prime} X_{1+k}\right) \cos \left(s^{\prime} X_{1}\right)\right\}-n u(r) u(s) \\
& =E\left\{\frac{1}{2}\left[\cos \left((r-s)^{\prime} X_{1}\right)+\cos \left((r+s)^{\prime} X_{1}\right)\right]\right\}+ \\
& +\frac{1}{n} \sum_{k=1}^{n-1}(n-k) E\left\{\frac{1}{2}\left[\cos \left(r^{\prime} X_{1}-s^{\prime} X_{1+k}\right)+\cos \left(r^{\prime} X_{1}+s^{\prime} X_{1+k}\right)\right]\right\}+ \\
& +\frac{1}{n} \sum_{k=1}^{n-1}(n-k) E\left\{\frac{1}{2}\left[\cos \left(r^{\prime} X_{1+k}-s^{\prime} X_{1}\right)+\cos \left(r^{\prime} X_{1+k}+s^{\prime} X_{1}\right)\right]\right\}-n u(r) u(s) \\
& =\frac{1}{2}\{u(r-s)+u(r+s)\}-n u(r) u(s)+ \\
& +\frac{1}{2 n} \sum_{k=1}^{n-1}(n-k)\left\{\operatorname{Re} \Lambda_{k}(r,-s)+\operatorname{Re} \Lambda_{k}(r, s)+\operatorname{Re} \Lambda_{k}(-s, r)+\operatorname{Re} \Lambda_{k}(s, r)\right\} \\
& =\frac{1}{2}\{\operatorname{Re} \varphi(r-s)+\operatorname{Re} \varphi(r+s)\}-n \operatorname{Re} \varphi(r) \operatorname{Re} \varphi(s)+ \\
& +\frac{1}{2 n} \sum_{k=1}^{n-1}(n-k)\left\{\operatorname{Re} \Lambda_{k}(r,-s)+\operatorname{Re} \Lambda_{k}(r, s)+\operatorname{Re} \Lambda_{k}(-s, r)+\operatorname{Re} \Lambda_{k}(s, r)\right\}
\end{aligned}
$$




$$
\begin{aligned}
& \operatorname{cov}\left[\operatorname{Re} Y_{n}(r), \operatorname{Im} Y_{n}(s)\right]=E\left[\operatorname{Re} Y_{n}(r) . \operatorname{Im} Y_{n}(s)\right]= \\
& =E\left\{\sqrt{n} \operatorname{Re}\left(\varphi_{n}(r)-\varphi(r)\right) \sqrt{n} \operatorname{Im}\left(\varphi_{n}(s)-\varphi(s)\right)\right\} \\
& =n E\left\{\operatorname{Re} \varphi_{n}(r) \operatorname{Im} \varphi_{n}(s)\right\}-n \operatorname{Re} \varphi(r) \operatorname{Im} \varphi(s) \\
& =n E\left\{u_{n}(r) v_{n}(s)\right\}-n u(r) v(s) \\
& =n E\left\{\frac{1}{n} \sum_{j=1}^{n} \cos \left(r^{\prime} X_{j}\right) \frac{1}{n} \sum_{k=1}^{n} \sin \left(s^{\prime} X_{k}\right)\right\}-n u(r) v(s) \\
& =\frac{1}{n} E\left\{\sum_{j=1}^{n} \cos \left(r^{\prime} X_{j}\right) \sin \left(s^{\prime} X_{j}\right)+\sum_{j=1}^{n} \sum_{\substack{k=1 \\
k \neq j}}^{n} \cos \left(r^{\prime} X_{j}\right) \sin \left(s^{\prime} X_{k}\right)\right\}-n u(r) v(s) \\
& =\frac{1}{n} \sum_{j=1}^{n} E\left\{\cos \left(r^{\prime} X_{j}\right) \sin \left(s^{\prime} X_{j}\right)\right\}+ \\
& +\frac{1}{n} \sum_{j=1}^{n} \sum_{\substack{k=1 \\
k \neq j}}^{n} E\left\{\cos \left(r^{\prime} X_{j}\right) \sin \left(s^{\prime} X_{k}\right)\right\}-n u(r) v(s) \\
& =\frac{1}{n} \sum_{j=1}^{n} E\left\{\cos \left(r^{\prime} X_{1}\right) \sin \left(s^{\prime} X_{1}\right)\right\}+\frac{1}{n} \sum_{k=1}^{n-1}(n-k) E\left\{\cos \left(r^{\prime} X_{1}\right) \sin \left(s^{\prime} X_{1+k}\right)\right\}+ \\
& +\frac{1}{n} \sum_{k=1}^{n-1}(n-k) E\left\{\cos \left(r^{\prime} X_{1+k}\right) \sin \left(s^{\prime} X_{1}\right)\right\}-n u(r) v(s) \\
& =E\left\{\cos \left(r^{\prime} X_{1}\right) \sin \left(s^{\prime} X_{1}\right)\right\}+\frac{1}{n} \sum_{k=1}^{n-1}(n-k) E\left\{\cos \left(r^{\prime} X_{1}\right) \sin \left(s^{\prime} X_{1+k}\right)\right\}+ \\
& +\frac{1}{n} \sum_{k=1}^{n-1}(n-k) E\left\{\cos \left(r^{\prime} X_{1+k}\right) \sin \left(s^{\prime} X_{1}\right)\right\}-n u(r) v(s) \\
& =E\left\{\frac{1}{2}\left[\sin \left((-r+s)^{\prime} X_{1}\right)+\sin \left((r+s)^{\prime} X_{1}\right)\right]\right\}+ \\
& +\frac{1}{n} \sum_{k=1}^{n-1}(n-k) E\left\{\frac{1}{2}\left[\sin \left(-r^{\prime} X_{1}+s^{\prime} X_{1+k}\right)+\sin \left(r^{\prime} X_{1}+s^{\prime} X_{1+k}\right)\right]\right\}+ \\
& +\frac{1}{n} \sum_{k=1}^{n-1}(n-k) E\left\{\frac{1}{2}\left[\sin \left(-r^{\prime} X_{1+k}+s^{\prime} X_{1}\right)+\sin \left(r^{\prime} X_{1+k}+s^{\prime} X_{1}\right)\right]\right\}-n u(r) v(s) \\
& =\frac{1}{2}\{v(-r+s)+v(r+s)\}-n u(r) v(s)+ \\
& +\frac{1}{2 n} \sum_{k=1}^{n-1}(n-k)\left\{\operatorname{Im} \Lambda_{k}(-r, s)+\operatorname{Im} \Lambda_{k}(r, s)+\operatorname{Im} \Lambda_{k}(s,-r)+\operatorname{Im} \Lambda_{k}(s, r)\right\} \\
& =\frac{1}{2}\{-\operatorname{Im} \varphi(r-s)+\operatorname{Im} \varphi(r+s)\}-n \operatorname{Re} \varphi(r) \operatorname{Im} \varphi(s)+ \\
& +\frac{1}{2 n} \sum_{k=1}^{n-1}(n-k)\left\{-\operatorname{Im} \Lambda_{k}(r,-s)+\operatorname{Im} \Lambda_{k}(r, s)-\operatorname{Im} \Lambda_{k}(-s, r)+\operatorname{Im} \Lambda_{k}(s, r)\right\}
\end{aligned}
$$




$$
\begin{aligned}
& \operatorname{cov}\left[\operatorname{Im} Y_{n}(r), \operatorname{Re} Y_{n}(s)\right]=E\left[\operatorname{Im} Y_{n}(r) \cdot \operatorname{Re} Y_{n}(s)\right]= \\
& =E\left\{\sqrt{n} \operatorname{Im}\left(\varphi_{n}(r)-\varphi(r)\right) \sqrt{n} \operatorname{Re}\left(\varphi_{n}(s)-\varphi(s)\right)\right\} \\
& =n E\left\{\operatorname{Im} \varphi_{n}(r) \operatorname{Re} \varphi_{n}(s)\right\}-n \operatorname{Im} \varphi(r) \operatorname{Re} \varphi(s) \\
& =n E\left\{v_{n}(r) u_{n}(s)\right\}-n v(r) u(s) \\
& =n E\left\{\frac{1}{n} \sum_{j=1}^{n} \sin \left(r^{\prime} X_{j}\right) \frac{1}{n} \sum_{k=1}^{n} \cos \left(s^{\prime} X_{k}\right)\right\}-n v(r) u(s) \\
& =\frac{1}{n} E\left\{\sum_{j=1}^{n} \sin \left(r^{\prime} X_{j}\right) \cos \left(s^{\prime} X_{j}\right)+\sum_{j=1}^{n} \sum_{\substack{k=1 \\
k \neq j}}^{n} \sin \left(r^{\prime} X_{j}\right) \cos \left(s^{\prime} X_{k}\right)\right\}-n v(r) u(s) \\
& =\frac{1}{n} \sum_{j=1}^{n} E\left\{\sin \left(r^{\prime} X_{j}\right) \cos \left(s^{\prime} X_{j}\right)\right\}+ \\
& +\frac{1}{n} \sum_{j=1}^{n} \sum_{\substack{k=1 \\
k \neq j}}^{n} E\left\{\sin \left(r^{\prime} X_{j}\right) \cos \left(s^{\prime} X_{k}\right)\right\}-n v(r) u(s) \\
& =\frac{1}{n} \sum_{j=1}^{n} E\left\{\sin \left(r^{\prime} X_{1}\right) \cos \left(s^{\prime} X_{1}\right)\right\}+\frac{1}{n} \sum_{k=1}^{n-1}(n-k) E\left\{\sin \left(r^{\prime} X_{1}\right) \cos \left(s^{\prime} X_{1+k}\right)\right\}+ \\
& +\frac{1}{n} \sum_{k=1}^{n-1}(n-k) E\left\{\sin \left(r^{\prime} X_{1+k}\right) \cos \left(s^{\prime} X_{1}\right)\right\}-n v(r) u(s) \\
& =E\left\{\sin \left(r^{\prime} X_{1}\right) \cos \left(s^{\prime} X_{1}\right)\right\}+\frac{1}{n} \sum_{k=1}^{n-1}(n-k) E\left\{\sin \left(r^{\prime} X_{1}\right) \cos \left(s^{\prime} X_{1+k}\right)\right\}+ \\
& +\frac{1}{n} \sum_{k=1}^{n-1}(n-k) E\left\{\sin \left(r^{\prime} X_{1+k}\right) \cos \left(s^{\prime} X_{1}\right)\right\}-n v(r) u(s) \\
& =E\left\{\frac{1}{2}\left[\sin \left((r-s)^{\prime} X_{1}\right)+\sin \left((r+s)^{\prime} X_{1}\right)\right]\right\}+ \\
& +\frac{1}{n} \sum_{k=1}^{n-1}(n-k) E\left\{\frac{1}{2}\left[\sin \left(r^{\prime} X_{1}-s^{\prime} X_{1+k}\right)+\sin \left(r^{\prime} X_{1}+s^{\prime} X_{1+k}\right)\right]\right\}+ \\
& +\frac{1}{n} \sum_{k=1}^{n-1}(n-k) E\left\{\frac{1}{2}\left[\sin \left(r^{\prime} X_{1+k}-s^{\prime} X_{1}\right)+\sin \left(r^{\prime} X_{1+k}+s^{\prime} X_{1}\right)\right]\right\}-n v(r) u(s) \\
& =\frac{1}{2}\{v(r-s)+v(r+s)\}-n v(r) u(s)+ \\
& +\frac{1}{2 n} \sum_{k=1}^{n-1}(n-k)\left\{\operatorname{Im} \Lambda_{k}(r,-s)+\operatorname{Im} \Lambda_{k}(r, s)+\operatorname{Im} \Lambda_{k}(-s, r)+\operatorname{Im} \Lambda_{k}(s, r)\right\} \\
& =\frac{1}{2}\{\operatorname{Im} \varphi(r-s)+\operatorname{Im} \varphi(r+s)\}-n \operatorname{Im} \varphi(r) \operatorname{Re} \varphi(s)+ \\
& +\frac{1}{2 n} \sum_{k=1}^{n-1}(n-k)\left\{\operatorname{Im} \Lambda_{k}(r,-s)+\operatorname{Im} \Lambda_{k}(r, s)+\operatorname{Im} \Lambda_{k}(-s, r)+\operatorname{Im} \Lambda_{k}(s, r)\right\}
\end{aligned}
$$




$$
\begin{aligned}
& \operatorname{cov}\left[\operatorname{Im} Y_{n}(r), \operatorname{Im} Y_{n}(s)\right]=E\left[\operatorname{Im} Y_{n}(r) . \operatorname{Im} Y_{n}(s)\right]= \\
& =E\left\{\sqrt{n} \operatorname{Im}\left(\varphi_{n}(r)-\varphi(r)\right) \sqrt{n} \operatorname{Im}\left(\varphi_{n}(s)-\varphi(s)\right)\right\} \\
& =n E\left\{\operatorname{Im} \varphi_{n}(r) \operatorname{Im} \varphi_{n}(s)\right\}-n \operatorname{Im} \varphi(r) \operatorname{Im} \varphi(s) \\
& =n E\left\{v_{n}(r) v_{n}(s)\right\}-n v(r) v(s) \\
& =n E\left\{\frac{1}{n} \sum_{j=1}^{n} \sin \left(r^{\prime} X_{j}\right) \frac{1}{n} \sum_{k=1}^{n} \sin \left(s^{\prime} X_{k}\right)\right\}-n v(r) v(s) \\
& =\frac{1}{n} E\left\{\sum_{j=1}^{n} \sin \left(r^{\prime} X_{j}\right) \sin \left(s^{\prime} X_{j}\right)+\sum_{j=1}^{n} \sum_{\substack{k=1 \\
k \neq j}}^{n} \sin \left(r^{\prime} X_{j}\right) \sin \left(s^{\prime} X_{k}\right)\right\}-n v(r) v(s) \\
& =\frac{1}{n} \sum_{j=1}^{n} E\left\{\sin \left(r^{\prime} X_{j}\right) \sin \left(s^{\prime} X_{j}\right)\right\}+ \\
& +\frac{1}{n} \sum_{j=1}^{n} \sum_{\substack{k=1 \\
k \neq j}}^{n} E\left\{\sin \left(r^{\prime} X_{j}\right) \sin \left(s^{\prime} X_{k}\right)\right\}-n v(r) v(s) \\
& =\frac{1}{n} \sum_{j=1}^{n} E\left\{\sin \left(r^{\prime} X_{1}\right) \sin \left(s^{\prime} X_{1}\right)\right\}+\frac{1}{n} \sum_{k=1}^{n-1}(n-k) E\left\{\sin \left(r^{\prime} X_{1}\right) \sin \left(s^{\prime} X_{1+k}\right)\right\}+ \\
& +\frac{1}{n} \sum_{k=1}^{n-1}(n-k) E\left\{\sin \left(r^{\prime} X_{1+k}\right) \sin \left(s^{\prime} X_{1}\right)\right\}-n v(r) v(s) \\
& =E\left\{\sin \left(r^{\prime} X_{1}\right) \sin \left(s^{\prime} X_{1}\right)\right\}+\frac{1}{n} \sum_{k=1}^{n-1}(n-k) E\left\{\sin \left(r^{\prime} X_{1}\right) \sin \left(s^{\prime} X_{1+k}\right)\right\}+ \\
& +\frac{1}{n} \sum_{k=1}^{n-1}(n-k) E\left\{\sin \left(r^{\prime} X_{1+k}\right) \sin \left(s^{\prime} X_{1}\right)\right\}-n v(r) v(s) \\
& =E\left\{\frac{1}{2}\left[\cos \left((r-s)^{\prime} X_{1}\right)-\cos \left((r+s)^{\prime} X_{1}\right)\right]\right\}+ \\
& +\frac{1}{n} \sum_{k=1}^{n-1}(n-k) E\left\{\frac{1}{2}\left[\cos \left(r^{\prime} X_{1}-s^{\prime} X_{1+k}\right)-\cos \left(r^{\prime} X_{1}+s^{\prime} X_{1+k}\right)\right]\right\}+ \\
& +\frac{1}{n} \sum_{k=1}^{n-1}(n-k) E\left\{\frac{1}{2}\left[\cos \left(r^{\prime} X_{1+k}-s^{\prime} X_{1}\right)-\cos \left(r^{\prime} X_{1+k}+s^{\prime} X_{1}\right)\right]\right\}-n v(r) v(s) \\
& =\frac{1}{2}\{u(r-s)-u(r+s)\}-n v(r) v(s)+ \\
& +\frac{1}{2 n} \sum_{k=1}^{n-1}(n-k)\left\{\operatorname{Re} \Lambda_{k}(r,-s)-\operatorname{Re} \Lambda_{k}(r, s)+\operatorname{Re} \Lambda_{k}(-s, r)-\operatorname{Re} \Lambda_{k}(s, r)\right\} \\
& =\frac{1}{2}\{\operatorname{Re} \varphi(r-s)-\operatorname{Re} \varphi(r+s)\}-n \operatorname{Im} \varphi(r) \operatorname{Im} \varphi(s)+ \\
& +\frac{1}{2 n} \sum_{k=1}^{n-1}(n-k)\left\{\operatorname{Re} \Lambda_{k}(r,-s)-\operatorname{Re} \Lambda_{k}(r, s)+\operatorname{Re} \Lambda_{k}(-s, r)-\operatorname{Re} \Lambda_{k}(s, r)\right\}
\end{aligned}
$$


Resumindo:

$$
\begin{aligned}
\Omega_{R R, n}(r, s)= & \frac{1}{2}\{\operatorname{Re} \varphi(r-s)+\operatorname{Re} \varphi(r+s)\}-n \operatorname{Re} \varphi(r) \operatorname{Re} \varphi(s)+ \\
& +\frac{1}{2 n} \sum_{k=1}^{n-1}(n-k)\left\{\operatorname{Re} \Lambda_{k}(r,-s)+\operatorname{Re} \Lambda_{k}(r, s)+\operatorname{Re} \Lambda_{k}(-s, r)+\operatorname{Re} \Lambda_{k}(s, r)\right\} \\
\Omega_{R I, n}(r, s)= & \frac{1}{2}\{-\operatorname{Im} \varphi(r-s)+\operatorname{Im} \varphi(r+s)\}-n \operatorname{Re} \varphi(r) \operatorname{Im} \varphi(s)+ \\
& +\frac{1}{2 n} \sum_{k=1}^{n-1}(n-k)\left\{-\operatorname{Im} \Lambda_{k}(r,-s)+\operatorname{Im} \Lambda_{k}(r, s)-\operatorname{Im} \Lambda_{k}(-s, r)+\operatorname{Im} \Lambda_{k}(s, r)\right\} \\
\Omega_{I R, n}(r, s)= & \frac{1}{2}\{\operatorname{Im} \varphi(r-s)+\operatorname{Im} \varphi(r+s)\}-n \operatorname{Im} \varphi(r) \operatorname{Re} \varphi(s)+ \\
& +\frac{1}{2 n} \sum_{k=1}^{n-1}(n-k)\left\{\operatorname{Im} \Lambda_{k}(r,-s)+\operatorname{Im} \Lambda_{k}(r, s)+\operatorname{Im} \Lambda_{k}(-s, r)+\operatorname{Im} \Lambda_{k}(s, r)\right\} \\
& \frac{1}{2}\{\operatorname{Re} \varphi(r-s)-\operatorname{Re} \varphi(r+s)\}-n \operatorname{Im} \varphi(r) \operatorname{Im} \varphi(s)+ \\
& +\frac{1}{2 n} \sum_{k=1}^{n-1}(n-k)\left\{\operatorname{Re} \Lambda_{k}(r,-s)-\operatorname{Re} \Lambda_{k}(r, s)+\operatorname{Re} \Lambda_{k}(-s, r)-\operatorname{Re} \Lambda_{k}(s, r)\right\}
\end{aligned}
$$

Assim, analogamente ao caso i.i.d. da seção anterior (eq. B.20), como $Y_{n}(r)=$ $\sqrt{n}\left(\varphi_{n}(r)-\varphi(r)\right)$ podemos escrever que

$$
\operatorname{cov}\left[\left(\begin{array}{c}
\operatorname{Re} \varphi_{n}(r) \\
\operatorname{Im} \varphi_{n}(r)
\end{array}\right),\left(\begin{array}{c}
\operatorname{Re} \varphi_{n}(s) \\
\operatorname{Im} \varphi_{n}(s)
\end{array}\right)\right]=\frac{1}{n} \Omega_{n}(r, s)
$$

onde $\Omega_{n}(r, s)=\left(\begin{array}{cc}\Omega_{R R, n}(r, s) & \Omega_{R I, n}(r, s) \\ \Omega_{I R, n}(r, s) & \Omega_{I I, n}(r, s)\end{array}\right)$ e os elementos da partição são dados pelas equações (B.33) a (B.36).

Vale notar que as equações (B.33) a (B.36) diferem das equações obtidas por Knight e Yu (2002), pg. 716, pois apresentam um $n$ multiplicando o produto das partes real ou imaginária de $\varphi_{z}(r)$ e $\varphi_{z}(s)$, além de diferentes sinais nos somatórios. Por outro lado, o resultado acima coincide com o apresentado em Knight e Satchell (1997), pg. 176, tomando-se o cuidado de notar que a nomenclatura $\Omega^{p}$ utilizada ali refere-se ao nosso $\Omega_{n}(r, s) / n$. 


\section{B.2.1 Convergência da seqüência $\Omega_{n}(r, s)$}

Vamos agora verificar se a seqüência $\Omega_{n}(r, s)$ converge quando $n \rightarrow \infty$. Para isso vamos assumir que a seqüência $\left\{Z_{j}\right\}$ seja $\alpha$-mixing (forte). Neste caso, temos que

$$
\left|E\left[\exp \left(i r^{\prime} Z_{j}+i s^{\prime} Z_{j+k}\right)\right]-E\left[\exp \left(i r^{\prime} Z_{j}\right)\right] \cdot E\left[\exp \left(i r^{\prime} Z_{j+k}\right)\right]\right|=\alpha(k) \underset{k \rightarrow \infty}{\rightarrow} 0
$$

Seja então $\Lambda_{\infty}(r, s)$ definido por:

$$
\begin{aligned}
\Lambda_{\infty}(r, s) & =\lim _{k \rightarrow \infty} \Lambda_{k}(r, s) \\
& =\lim _{k \rightarrow \infty} E\left[\exp \left(i r^{\prime} Z_{j}+i s^{\prime} Z_{j+k}\right)\right] \\
& =E\left[\exp \left(i r^{\prime} Z_{j}\right)\right] \cdot E\left[\exp \left(i r^{\prime} Z_{j+k}\right)\right] \\
& =\varphi(r) \varphi(s)
\end{aligned}
$$

Podemos escrever $\Lambda_{\infty}(r, s)=\varphi(r) \varphi(s)$ como

$$
\begin{aligned}
\varphi(r) \varphi(s)= & (u(r)+i v(r))(u(s)+i v(s)) \\
= & u(r) u(s)+i u(r) v(s)+ \\
& +i v(r) u(s)-v(r) v(s) \\
= & u(r) u(s)-v(r) v(s)+ \\
& +i(u(r) v(s)+v(r) u(s))
\end{aligned}
$$

Logo

$$
\begin{aligned}
\operatorname{Re}[\varphi(r) \varphi(s)] & =u(r) u(s)-v(r) v(s) \\
\operatorname{Im}[\varphi(r) \varphi(s)] & =u(r) v(s)+v(r) u(s)
\end{aligned}
$$

Para o caso de $\Omega_{R R, n}(r, s)$ obtemos:

$$
\begin{aligned}
& \operatorname{Re} \Lambda_{\infty}(r, s)+\operatorname{Re} \Lambda_{\infty}(r,-s)+\operatorname{Re} \Lambda_{\infty}(s, r)+\operatorname{Re} \Lambda_{\infty}(s,-r) \\
= & \operatorname{Re} \varphi(r) \varphi(s)+\operatorname{Re} \varphi(r) \varphi(-s)+\operatorname{Re} \varphi(r) \varphi(s)+\operatorname{Re} \varphi(-r) \varphi(s) \\
= & u(r) u(s)-v(r) v(s)+ \\
& +u(r) u(-s)-v(r) v(-s)+ \\
& +u(r) u(s)-v(r) v(s)+ \\
& +u(-r) u(s)-v(-r) v(s) \\
= & u(r) u(s)-v(r) v(s)+ \\
& +u(r) u(s)+v(r) v(s)+ \\
& +u(r) u(s)-v(r) v(s)+ \\
& +u(r) u(s)+v(r) v(s) \\
= & 4 u(r) u(s)
\end{aligned}
$$


Para o caso de $\Omega_{R I, n}(r, s)$ obtemos:

$$
\begin{aligned}
& \operatorname{Im} \Lambda_{\infty}(-r, s)+\operatorname{Im} \Lambda_{\infty}(r, s)+\operatorname{Im} \Lambda_{\infty}(s,-r)+\operatorname{Im} \Lambda_{\infty}(s, r) \\
= & \operatorname{Im} \varphi(-r) \varphi(s)+\operatorname{Im} \varphi(r) \varphi(s)+\operatorname{Im} \varphi(-r) \varphi(s)+\operatorname{Im} \varphi(r) \varphi(s) \\
= & u(-r) v(s)+v(-r) u(s)+ \\
& +u(r) v(s)+v(r) u(s)+ \\
& +u(-r) v(s)+v(-r) u(s)+ \\
& +u(r) v(s)+v(r) u(s) \\
= & u(r) v(s)-v(r) u(s)+ \\
& +u(r) v(s)+v(r) u(s)+ \\
& +u(r) v(s)-v(r) u(s)+ \\
& +u(r) v(s)+v(r) u(s) \\
= & 4 u(r) v(s)
\end{aligned}
$$

Para o caso de $\Omega_{I I, n}(r, s)$ obtemos:

$$
\begin{aligned}
& \operatorname{Re} \Lambda_{\infty}(r,-s)-\operatorname{Re} \Lambda_{\infty}(r, s)+\operatorname{Re} \Lambda_{\infty}(s,-r)-\operatorname{Re} \Lambda_{\infty}(s, r) \\
= & \operatorname{Re} \varphi(r) \varphi(-s)-\operatorname{Re} \varphi(r) \varphi(s)+\operatorname{Re} \varphi(-r) \varphi(s)-\operatorname{Re} \varphi(r) \varphi(s) \\
= & u(r) u(-s)-v(r) v(-s)- \\
& -u(r) u(s)+v(r) v(s)+ \\
& +u(-r) u(s)-v(-r) v(s)- \\
& -u(r) u(s)+v(r) v(s) \\
= & u(r) u(s)+v(r) v(s)- \\
& -u(r) u(s)+v(r) v(s)+ \\
& +u(r) u(s)+v(r) v(s)- \\
& -u(r) u(s)+v(r) v(s) \\
= & 4 v(r) v(s)
\end{aligned}
$$

Seja agora um $m>n_{0}$ suficientemente grande tal que $\left|\Lambda_{m}(r, s)-\varphi(r) \varphi(s)\right|<\varepsilon$. Neste 
caso, para $\Omega_{R R, m}(r, s)$ podemos quebrar o somatório em (B.33) da seguinte maneira:

$$
\begin{aligned}
& \Omega_{R R, m}(r, s) \\
= & \frac{1}{2}\{\operatorname{Re} \varphi(r-s)+\operatorname{Re} \varphi(r+s)\}-m \operatorname{Re} \varphi(r) \operatorname{Re} \varphi(s)+ \\
& +\frac{1}{2 m} \sum_{k=1}^{n_{0}-1}(m-k)\left\{\operatorname{Re} \Lambda_{k}(r,-s)+\operatorname{Re} \Lambda_{k}(r, s)+\operatorname{Re} \Lambda_{k}(-s, r)+\operatorname{Re} \Lambda_{k}(s, r)\right\} \\
& +\frac{1}{2 m} \sum_{k=n_{0}}^{m-1}(m-k)\left\{\operatorname{Re} \Lambda_{k}(r,-s)+\operatorname{Re} \Lambda_{k}(r, s)+\operatorname{Re} \Lambda_{k}(-s, r)+\operatorname{Re} \Lambda_{k}(s, r)\right\} .
\end{aligned}
$$

Vamos agora obter o elemento seguinte $\Omega_{R R, m+1}(r, s)$ da seqüência:

$$
\begin{aligned}
& \Omega_{R R, m+1}(r, s) \\
= & \frac{1}{2}\{\operatorname{Re} \varphi(r-s)+\operatorname{Re} \varphi(r+s)\}-(m+1) \operatorname{Re} \varphi(r) \operatorname{Re} \varphi(s)+ \\
& +\frac{1}{2(m+1)} \sum_{k=1}^{n_{0}-1}(m+1-k)\left\{\operatorname{Re} \Lambda_{k}(r,-s)+\operatorname{Re} \Lambda_{k}(r, s)+\operatorname{Re} \Lambda_{k}(-s, r)+\operatorname{Re} \Lambda_{k}(s, r)\right\} \\
& +\frac{1}{2(m+1)} \sum_{k=n_{0}}^{m}(m+1-k)\left\{\operatorname{Re} \Lambda_{k}(r,-s)+\operatorname{Re} \Lambda_{k}(r, s)+\operatorname{Re} \Lambda_{k}(-s, r)+\operatorname{Re} \Lambda_{k}(s, r)\right\} .
\end{aligned}
$$

Chamemos

$$
\Delta_{k}(r, s)=\operatorname{Re} \Lambda_{k}(r,-s)+\operatorname{Re} \Lambda_{k}(r, s)+\operatorname{Re} \Lambda_{k}(-s, r)+\operatorname{Re} \Lambda_{k}(s, r) .
$$

Fazendo agora a diferença entre $\Omega_{R R, m+1}(r, s)$ e $\Omega_{R R, m}(r, s)$ obtemos

$$
\begin{aligned}
& \Omega_{R R, m+1}(r, s)-\Omega_{R R, m}(r, s) \\
= & -u(r) u(s)+ \\
& +\frac{1}{2}\left\{\left(\frac{m}{m+1}-\frac{m-1}{m}\right) \Delta_{1}(r, s)+\left(\frac{m-1}{m+1}-\frac{m-2}{m}\right) \Delta_{2}(r, s)+\cdots+\right. \\
& +\left(\frac{m+2-n_{0}}{m+1}-\frac{m+1-n_{0}}{m}\right) \Delta_{n_{0}-1}(r, s)+\left(\frac{m+1-n_{0}}{m+1}-\frac{m-n_{0}}{m}\right) \Delta_{n_{0}}(r, s)+\cdots+ \\
& \left.+\left(\frac{2}{m+1}-\frac{1}{m}\right) \Delta_{m-1}(r, s)+\frac{1}{m+1} \Delta_{m}(r, s)\right\} \\
= & -u(r) u(s)+ \\
& +\frac{1}{2}\left\{\frac{1}{m(m+1)} \Delta_{1}(r, s)+\frac{2}{m(m+1)} \Delta_{2}(r, s)+\cdots\right. \\
& +\frac{n_{0}-1}{m(m+1)} \Delta_{n_{0}-1}(r, s)+\frac{n_{0}}{m(m+1)} \Delta_{n_{0}}(r, s)+\cdots+ \\
& \left.+\frac{m-1}{m(m+1)} \Delta_{m-1}(r, s)+\frac{m}{m(m+1)} \Delta_{m}(r, s)\right\} .
\end{aligned}
$$

Sejam

$$
\Delta_{\max }(r, s)=\max \left\{\Delta_{1}(r, s), \Delta_{2}(r, s), \cdots, \Delta_{n_{0}}(r, s)\right\}
$$


e

$$
\Delta_{\min }(r, s)=\min \left\{\Delta_{1}(r, s), \Delta_{2}(r, s), \cdots, \Delta_{n_{0}}(r, s)\right\} .
$$

Podemos escrever

$$
\begin{aligned}
& \Omega_{R R, m+1}(r, s)-\Omega_{R R, m}(r, s) \\
\leq & -u(r) u(s)+\frac{1}{2} \frac{\left(1+2+\cdots+n_{0}-1\right)}{m(m+1)} \Delta_{\max }(r, s)+ \\
& +\frac{n_{0}}{m(m+1)} \Delta_{n_{0}}(r, s)+\cdots+\frac{m}{m(m+1)} \Delta_{m}(r, s) \\
& \Omega_{R R, m+1}(r, s)-\Omega_{R R, m}(r, s) \\
\geq & -u(r) u(s)+\frac{1}{2} \frac{\left(1+2+\cdots+n_{0}-1\right)}{m(m+1)} \Delta_{\min }(r, s)+ \\
& +\frac{n_{0}}{m(m+1)} \Delta_{n_{0}}(r, s)+\cdots+\frac{m}{m(m+1)} \Delta_{m}(r, s) .
\end{aligned}
$$

Como $\left|\Lambda_{k}(r, s)-\varphi(r) \varphi(s)\right|<\varepsilon$ para $k>n_{0}$, temos por (B.41) que $\left|\Delta_{k}(r, s)-4 u(r) u(s)\right|<4 \varepsilon$ para $k>n_{0}$. Assim

$$
\begin{aligned}
& \Omega_{R R, m+1}(r, s)-\Omega_{R R, m}(r, s) \\
& \leq-u(r) u(s)+\frac{1}{4} \frac{n_{0}\left(n_{0}-1\right)}{m(m+1)} \Delta_{\max }(r, s)+ \\
& +\frac{1}{2}\left(\frac{n_{0}}{m(m+1)}+\cdots+\frac{m}{m(m+1)}\right) 4(u(r) u(s)+\varepsilon) \\
& \Omega_{R R, m+1}(r, s)-\Omega_{R R, m}(r, s) \\
& \geq-u(r) u(s)+\frac{1}{4} \frac{n_{0}\left(n_{0}-1\right)}{m(m+1)} \Delta_{\min }(r, s)+ \\
& +\frac{1}{2}\left(\frac{n_{0}}{m(m+1)}+\cdots+\frac{m}{m(m+1)}\right) 4(u(r) u(s)-\varepsilon) . \\
& \Omega_{R R, m+1}(r, s)-\Omega_{R R, m}(r, s) \\
& \leq-u(r) u(s)+\frac{1}{4} \frac{n_{0}\left(n_{0}-1\right)}{m(m+1)} \Delta_{\max }(r, s)+ \\
& +\frac{m(m+1)-n_{0}\left(n_{0}-1\right)}{m(m+1)}(u(r) u(s)+\varepsilon) \\
& \Omega_{R R, m+1}(r, s)-\Omega_{R R, m}(r, s) \\
& \geq-u(r) u(s)+\frac{1}{4} \frac{n_{0}\left(n_{0}-1\right)}{m(m+1)} \Delta_{\min }(r, s)+ \\
& +\frac{m(m+1)-n_{0}\left(n_{0}-1\right)}{m(m+1)}(u(r) u(s)-\varepsilon) .
\end{aligned}
$$




$$
\begin{aligned}
& \Omega_{R R, m+1}(r, s)-\Omega_{R R, m}(r, s) \\
\leq & -\frac{n_{0}\left(n_{0}-1\right)}{m(m+1)} u(r) u(s)+\frac{1}{4} \frac{n_{0}\left(n_{0}-1\right)}{m(m+1)} \Delta_{\max }(r, s)-\frac{n_{0}\left(n_{0}-1\right)}{m(m+1)} \varepsilon+\varepsilon \\
& \Omega_{R R, m+1}(r, s)-\Omega_{R R, m}(r, s) \\
\geq & -\frac{n_{0}\left(n_{0}-1\right)}{m(m+1)} u(r) u(s)+\frac{1}{4} \frac{n_{0}\left(n_{0}-1\right)}{m(m+1)} \Delta_{\min }(r, s)+\frac{n_{0}\left(n_{0}-1\right)}{m(m+1)} \varepsilon-\varepsilon .
\end{aligned}
$$

Note agora que quando $m \rightarrow \infty$, fixado $n_{0}$, as três primeiras parcelas tendem a zero. Assim fixados $\varepsilon>0$ e $n_{0}$, é possível encontrar um $m_{0}$ tal que para todo $m>m_{0}$

$$
\left|\Omega_{R R, m+1}(r, s)-\Omega_{R R, m}(r, s)\right| \leq \varepsilon .
$$

Logo a seqüência $\Omega_{R R, n}(r, s)$ é Cauchy e, portanto, convergente (Lima (2002)). Naturalmente o mesmo exercício poderia ser feito para $\Omega_{R I, n}(r, s), \Omega_{I R, n}(r, s)$ e $\Omega_{I I, n}(r, s)$, usando (B.42) a (B.43), e obtendo-se resultados análogos. 



\section{Apêndice $\mathrm{C}$}

\section{Demonstrações dos Capítulos 4 e 5}

Demonstração da proposição 4.1. O processo definido em (4.5) constitui um processo $\operatorname{AR}(1)$. A hipótese de $|\phi|<1$ implica que a variável latente $\psi_{j}$ é estacionária. Se denotarmos por $B$ o operador backward shift podemos escrever o processo para $\psi_{j}$ da seguinte forma:

$$
(1-\phi B) \psi_{j}=\delta+\sigma_{\eta} \eta_{j}
$$

Assim podemos escrever $\psi_{j}$ como

$$
\begin{aligned}
\psi_{j} & =(1-\phi B)^{-1}\left(\delta+\sigma_{\eta} \eta_{j}\right) \\
& =\left(1+\phi B+\phi^{2} B^{2}+\phi^{3} B^{3}+\cdots\right)\left(\delta+\sigma_{\eta} \eta_{j}\right) \\
& =\delta+\sigma_{\eta} \eta_{j}+\phi \delta+\phi \sigma_{\eta} \eta_{j-1}+\phi^{2} \delta+\phi^{2} \sigma_{\eta} \eta_{j-2}+\cdots \\
& =\left(\sum_{l=1}^{\infty} \phi^{l-1}\right) \delta+\sigma_{\eta} \sum_{l=0}^{\infty} \phi^{l} \eta_{j-l} \\
& =\frac{\delta}{1-\phi}+\sigma_{\eta} \sum_{l=0}^{\infty} \phi^{l} \eta_{j-l} .
\end{aligned}
$$

Como os $\eta_{j}$ são i.i.d. $\mathrm{N}(0,1)$, podemos calcular a média e a variância de $\psi_{j}$ por

$$
\begin{aligned}
E\left[\psi_{j}\right] & =\frac{\delta}{1-\phi}+\sigma_{\eta} \sum_{l=0}^{\infty} \phi^{l} E\left[\eta_{j-l}\right] \\
= & \frac{\delta}{1-\phi} \\
\operatorname{Var}\left[\psi_{j}\right] & =\sigma_{\eta}^{2} \sum_{l=0}^{\infty} \phi^{2 l} \operatorname{Var}\left[\eta_{j-l}\right] \\
& =\sigma_{\eta}^{2} \sum_{l=0}^{\infty} \phi^{2 l} \\
& =\frac{\sigma_{\eta}^{2}}{1-\phi^{2}}
\end{aligned}
$$


em que a passagem da penúltima para a última linha decorre de $|\phi|<1$. Como $\psi_{j}$ é um somatório de v.a. normais independentes, então também possui distribuição normal com média e variância dadas acima.

Demonstração da proposição 4.2. Usando (4.13) e (4.15) e a hipótese de que $|\phi|<1$ obtemos:

$$
\begin{aligned}
\varphi_{\psi}(t) & =E\left[\exp \left(i t \psi_{j}\right)\right]= \\
& =E\left\{\exp \left[i t \frac{\delta}{1-\phi}+i t \sigma_{\eta} \sum_{l=0}^{\infty} \phi^{l} \eta_{j-l}\right]\right\} \\
& =\exp \left(\frac{i t \delta}{1-\phi}\right) \prod_{l=0}^{\infty} E\left\{\exp \left[i t \sigma_{\eta} \phi^{l} \eta_{j-l}\right]\right\} \\
& =\exp \left(\frac{i t \delta}{1-\phi}\right) \prod_{l=0}^{\infty} \exp \left(-\frac{\sigma_{\eta}^{2} t^{2}}{2} \phi^{2 l}\right) \\
& =\exp \left(\frac{i t \delta}{1-\phi}\right) \exp \left(-\frac{\sigma_{\eta}^{2} t^{2}}{2} \sum_{l=0}^{\infty} \phi^{2 l}\right) \\
& =\exp \left(\frac{i t \delta}{1-\phi}-\frac{\sigma_{\eta}^{2} t^{2}}{2}\left(\frac{1}{1-\phi^{2}}\right)\right) .
\end{aligned}
$$

Demonstração da proposição 4.3. Para facilitar a notação seja $a=E[\zeta]$, de onde temos $\xi=\zeta / a$. Queremos obter

$$
F_{\Xi}(\xi)=\operatorname{Pr}(\Xi \leq \xi)=\operatorname{Pr}\left(\frac{\zeta}{a} \leq \xi\right)=\operatorname{Pr}(\zeta \leq a \xi)=F_{\zeta}(a \xi)
$$

Derivando ambos os lados da equação acima em relação a $\xi$ obtemos densidade de $\xi$ :

$$
f_{\xi}(\xi)=\frac{d F_{\xi}(\xi)}{d \xi}=\frac{d F_{\zeta}(a \xi)}{d \zeta} \frac{d \zeta(a \xi)}{d \xi}=f_{\zeta}(a \xi) a .
$$

Para o caso da distribuição Weibull $a=\Gamma\left(1+\frac{1}{\lambda}\right)$. Substituindo $f_{\zeta}$ por (4.9) em (C.1) obtemos

$$
f_{\xi}(\xi)=\left\{\begin{array}{c}
\lambda(a \xi)^{\lambda-1} e^{-(a \xi)^{\lambda}} a, \text { se } a \xi \geq 0 \\
0, \text { se } a \xi<0
\end{array}\right.
$$

que pode ser escrita

$$
f_{\xi}(\xi)=\left\{\begin{array}{c}
\lambda\left[\Gamma\left(1+\frac{1}{\lambda}\right)\right]^{\lambda} \xi^{\lambda-1} e^{-\left[\Gamma\left(1+\frac{1}{\lambda}\right) \xi\right]^{\lambda}}, \text { se } \xi \geq 0 \\
0, \text { se } \xi<0
\end{array} .\right.
$$

Para o caso da distribuição gama $a=\frac{\Gamma(\lambda+1)}{\Gamma(\lambda)}$. Mas, uma das propriedades da função gama é que

$$
\Gamma(x+1)=x \Gamma(x) .
$$


Assim $a=\frac{\Gamma(\lambda+1)}{\Gamma(\lambda)}$ pode ser simplificado para $a=\lambda$. Substituindo $f_{\zeta}$ por (4.11) em (C.1) obtemos

$$
f_{\xi}(\xi)=\left\{\begin{array}{c}
\frac{1}{\Gamma(\lambda)}(\lambda \xi)^{\lambda-1} e^{-\lambda \xi} \lambda, \text { se } \lambda \xi \geq 0 \\
0, \text { se } \lambda \xi<0
\end{array}\right.
$$

que pode ser escrita

$$
f_{\xi}(\xi)=\left\{\begin{array}{c}
\frac{\lambda^{\lambda}}{\Gamma(\lambda)} \xi^{\lambda-1} e^{-\lambda \xi}, \text { se } \xi \geq 0 \\
0, \text { se } \xi<0
\end{array}\right.
$$

Demonstração da proposição 4.4. Podemos obter o resultado facilmente se utilizarmos o resultado (C.1), com $a=1 / e^{\psi_{j}}$.Para o caso da distribuição Weibull obtemos

$$
f\left(x_{j} \mid \psi_{j}\right)=\left\{\begin{array}{c}
\lambda\left[\Gamma\left(1+\frac{1}{\lambda}\right)\right]^{\lambda}\left(\frac{x_{j}}{e^{\psi_{j}}}\right)^{\lambda-1} e^{-\left[\Gamma\left(1+\frac{1}{\lambda}\right) \frac{x_{j}}{e^{\psi_{j}}}\right]^{\lambda}} \frac{1}{e^{\psi_{j}}}, \text { se } x_{j} \geq 0 . \\
0, \text { se } x_{j}<0
\end{array}\right.
$$

Para o caso da distribuição gama

$$
f\left(x_{j} \mid \psi_{j}\right)=\left\{\begin{array}{c}
\frac{\lambda^{\lambda}}{\Gamma(\lambda)}\left(\frac{x_{j}}{e^{\psi_{j}}}\right)^{\lambda-1} e^{-\lambda \frac{x_{j}}{e^{\psi_{j}}}} \frac{1}{e^{\psi_{j}}}, \text { se } x_{j} \geq 0 \\
0, \text { se } x_{j}<0
\end{array} .\right.
$$

Caso $\lambda=1$ em qualquer das distribuições acima, obtemos o caso da distribuição exponencial

$$
f\left(x_{j} \mid \psi_{j}\right)=\left\{\begin{array}{c}
e^{-x_{j} e^{-\psi_{j}}} e^{-\psi_{j}}, \text { se } x_{j} \geq 0 \\
0, \text { se } x_{j}<0
\end{array} .\right.
$$

Demonstração da proposição 4.5. Chamemos $a=\lambda$ e $c=\frac{\Gamma\left(1+\lambda^{-1}\right)}{e^{\psi_{j}}}$. Assim,

(a) Exponencial:

$$
\begin{gathered}
f(x)=a e^{-a x} \quad x>0 \\
F(x)=\int_{0}^{x} a e^{-a t} d t=-\left.\frac{a}{a} e^{-a t}\right|_{0} ^{x}=-e^{-a x}+1 \\
F_{X_{j} \mid \Psi_{j}}\left(x_{j}\right)=1-e^{-x_{j} e^{-\psi_{j}}}
\end{gathered}
$$

Podemos simular $x_{j}$ dado $\psi_{j}$ chamando $F_{X_{j} \mid \Psi_{j}}\left(x_{j}\right)=u, u \sim U([0,1])$ e invertendo a eq. (C.2) acima:

$$
\begin{gathered}
\ln (1-u)=-e^{-\psi_{j}} x_{j} \\
x_{j}=-\frac{\ln (1-u)}{e^{-\psi_{j}}}
\end{gathered}
$$

(b) Weibull:

$$
f(x)=a c^{a} x^{a-1} e^{-(c x)^{a}}
$$




$$
F(x)=\int_{0}^{x} a c^{a} t^{a-1} e^{-(c t)^{a}} d t=a c^{a} \int_{0}^{x} t^{a-1} e^{-c^{a} t^{a}} d t
$$

Chamando $e^{-c^{a} t^{a}}=e^{-y} \Leftrightarrow-c^{a} t^{a}=-y \Rightarrow c^{a} a t^{a-1} d t=d y$ e substituindo na eq. acima:

$$
F(x)=\int_{0}^{c^{a} x^{a}} e^{-y} d y=1-e^{-(c x)^{a}}
$$

Para o nosso caso, $a=\lambda$ e $c=\frac{\Gamma\left(1+\lambda^{-1}\right)}{\exp \left(\psi_{j}\right)}$, de onde obtemos:

$$
F_{X_{j} \mid \Psi_{j}}\left(x_{j}\right)=1-\exp \left\{-\left[\frac{\Gamma\left(1+\lambda^{-1}\right)}{\exp \left(\psi_{j}\right)} x_{j}\right]^{\lambda}\right\}
$$

Podemos simular $x_{j}$ dado $\psi_{j}$ invertendo a eq. acima:

$$
\begin{gathered}
{[-\ln (1-u)]^{1 / \lambda}=\frac{\Gamma\left(1+\lambda^{-1}\right)}{\exp \left(\psi_{j}\right)} x_{j}} \\
x_{j}=\frac{\exp \left(\psi_{j}\right)[-\ln (1-u)]^{1 / \lambda}}{\Gamma\left(1+\lambda^{-1}\right)}
\end{gathered}
$$

(c) Gama:

$$
\begin{gathered}
f(x)=\frac{1}{\Gamma(a)}\left(\frac{a}{c}\right)^{a} x^{a-1} e^{-\frac{a}{c} x} \\
F(x)=\int_{0}^{x} \frac{1}{\Gamma(a)}\left(\frac{a}{c}\right)^{a} t^{a-1} e^{-\frac{a}{c} t} d t
\end{gathered}
$$

Chamando $y=\frac{a}{c} t \Rightarrow d y=\frac{a}{c} d t$ e substituindo acima:

$$
F(x)=\int_{0}^{\frac{a x}{c}} \frac{1}{\Gamma(a)}\left(\frac{a}{c}\right)^{a-1}\left(\frac{c y}{a}\right)^{a-1} e^{-y} d y=\frac{1}{\Gamma(a)} \int_{0}^{\frac{a x}{c}} y^{a-1} e^{-y} d y
$$

A integral acima é a função gama incompleta $\Gamma_{a x / c}(a)^{1}$. Assim, podemos escrever:

$$
F_{X_{j} \mid \Psi_{j}}\left(x_{j}\right)=\frac{\Gamma_{a x_{j} / c}(a)}{\Gamma(a)}
$$

\footnotetext{
${ }^{1}$ Ver por exemplo Abramowitz e Stegun (1972).
} 
Demonstração da proposição 4.6. Note inicialmente que a função inversa de $y=\ln x$, $x=e^{y}$, é monótona crescente; assim temos:

$$
F_{Y}(y)=\operatorname{Pr}[Y \leq y]=\operatorname{Pr}[\ln X \leq y]=\operatorname{Pr}\left[X \leq e^{y}\right]=F_{X}\left(e^{y}\right)
$$

Derivando a eq. acima em relação a y obtemos

$$
f_{Y}(y)=\frac{d F_{y}(y)}{d y}=\frac{d F_{x}\left(e^{y}\right)}{d x} \frac{d x\left(e^{y}\right)}{d y}=f_{X}\left(e^{y}\right) e^{y}
$$

Aplicando este resultado nas equações (4.24) a (4.26) obtemos:

\section{- Exponencial:}

$$
\begin{aligned}
p\left(y_{j} \mid \psi_{j}\right) & =e^{-e^{y_{j}} e^{-\psi_{j}}} e^{-\psi_{j}} e^{y_{j}} \\
& =e^{y_{j}-\psi_{j}-e^{y_{j}-\psi_{j}}} .
\end{aligned}
$$

- Weibull:

$$
\begin{aligned}
p\left(y_{j} \mid \psi_{j}\right) & =\lambda\left(\frac{\Gamma\left(1+\lambda^{-1}\right)}{e^{\psi_{j}}}\right)^{\lambda} e^{(\lambda-1) y_{j}-\left[\frac{\Gamma\left(1+\lambda^{-1}\right)}{e^{\psi_{j}}} e^{y_{j}}\right]^{\lambda}} e^{y_{j}} \\
& =\lambda\left[\Gamma\left(1+\lambda^{-1}\right)\right]^{\lambda} e^{\lambda\left(y_{j}-\psi_{j}\right)-\left[\Gamma\left(1+\lambda^{-1}\right)\right]^{\lambda} e^{\lambda\left(y_{j}-\psi_{j}\right)}}
\end{aligned}
$$

\section{- Gama:}

$$
\begin{aligned}
p\left(y_{j} \mid \psi_{j}\right) & =\frac{1}{\Gamma(\lambda)}\left(\frac{\lambda}{e^{\psi_{j}}}\right)^{\lambda} e^{(\lambda-1) y_{j}} e^{-\frac{\lambda}{e^{\psi_{j}}} e^{y_{j}}} e^{y_{j}} \\
& =\frac{\lambda^{\lambda}}{\Gamma(\lambda)} e^{\lambda\left(y_{j}-\psi_{j}\right)-\lambda e^{y_{j}-\psi_{j}}}
\end{aligned}
$$

Demonstração da proposição 4.8. Calculando a função característica para cada distribuição obtemos:

\section{- Log-exponencial:}

$$
\varphi_{\varepsilon}(t)=E\left[e^{i t \varepsilon}\right]=\int_{-\infty}^{\infty} e^{i t x} e^{x-e^{x}} d x=\int_{-\infty}^{\infty} e^{x(i t+1)} e^{-e^{x}} d x
$$

Chamando $y=e^{x}$, temos que $d y=e^{x} d x$, e substituindo na eq. acima obtemos:

$$
\int_{-\infty}^{\infty} e^{x(i t+1)} e^{-e^{x}} d x=\int_{0}^{\infty} y^{i t+1} e^{-y} d y
$$


A integral acima é basicamente a função gama com argumento complexo:

$$
\varphi_{\varepsilon}(t)=\Gamma(1+i t)
$$

\section{- Log-Weibull:}

$$
\varphi_{\varepsilon}(t)=E\left[e^{i t \varepsilon}\right]=\int_{-\infty}^{\infty} e^{i t x} \lambda\left[\Gamma\left(1+\lambda^{-1}\right)\right]^{\lambda} e^{\lambda x-\left[\Gamma\left(1+\lambda^{-1}\right)\right]^{\lambda} e^{\lambda x}} d x
$$

Chamando $e^{y}=\left[\Gamma\left(1+\lambda^{-1}\right)\right]^{\lambda} e^{\lambda x} \Leftrightarrow y=\lambda \ln \left[\Gamma\left(1+\lambda^{-1}\right)\right]+\lambda x \Rightarrow d y=\lambda d x$. Substituindo na eq. acima obtemos:

$$
\varphi_{\varepsilon}(t)=\int_{-\infty}^{\infty}\left(\frac{e^{y}}{\left[\Gamma\left(1+\lambda^{-1}\right)\right]^{\lambda}}\right)^{\frac{i t}{\lambda}} e^{y} e^{-e^{y}} d y=\frac{1}{\left[\Gamma\left(1+\lambda^{-1}\right)\right]^{i t}} \int_{-\infty}^{\infty} e^{y\left(\frac{i t}{\lambda}+1\right)-e^{y}} d y
$$

Utilizando a mesma transformação que no caso anterior $\left(z=e^{y}\right)$ obtemos:

$$
\varphi_{\varepsilon}(t)=\frac{\Gamma\left(1+\frac{i t}{\lambda}\right)}{\left[\Gamma\left(1+\lambda^{-1}\right)\right]^{i t}}
$$

\section{- Log-gama:}

$$
\varphi_{\varepsilon}(t)=E\left[e^{i t \varepsilon}\right]=\int_{-\infty}^{\infty} e^{i t x} \frac{\lambda^{\lambda}}{\Gamma(\lambda)} e^{\lambda x-\lambda e^{x}} d x=\frac{\lambda^{\lambda}}{\Gamma(\lambda)} \int_{-\infty}^{\infty} e^{x(i t+\lambda)-\lambda e^{x}} d x
$$

Chamando $\lambda e^{x}=e^{y} \Leftrightarrow d x=d y$ temos que:

$$
\begin{gathered}
\varphi_{\varepsilon}(t)=\frac{\lambda^{\lambda}}{\Gamma(\lambda)} \int_{-\infty}^{\infty}\left(\frac{e^{y}}{\lambda}\right)^{i t+\lambda} e^{-e^{y}} d y=\frac{1}{\lambda^{i t} \Gamma(\lambda)} \int_{-\infty}^{\infty} e^{y(i t+\lambda)-e^{y}} d y \\
\varphi_{\varepsilon}(t)=\frac{\Gamma(\lambda+i t)}{\lambda^{i t} \Gamma(\lambda)}
\end{gathered}
$$

Demonstração da proposição 4.9. Para calcular a função geratriz de cumulantes basta calcular o ln da função característica.

\section{- Log-exponencial:}

$$
G_{\varepsilon}(t)=\ln \Gamma(1+i t) .
$$

Derivando em relação a $t$ obtemos

$$
G_{\varepsilon}^{\prime}(t)=\frac{\Gamma^{\prime}(1+i t)}{\Gamma(1+i t)} i=i \Psi(1+i t)
$$




$$
G_{\varepsilon}^{(r)}(t)=i^{r} \Psi^{(r-1)}(1+i t), r>1
$$

- Log-Weibull:

$$
\begin{aligned}
G_{\varepsilon}(t) & =\ln \left\{\frac{\Gamma\left(1+\frac{i t}{\lambda}\right)}{\left[\Gamma\left(1+\lambda^{-1}\right)\right]^{i t}}\right\} \\
& =\ln \Gamma\left(1+i t \lambda^{-1}\right)-i t \ln \Gamma\left(1+\lambda^{-1}\right) .
\end{aligned}
$$

Derivando em relação a $t$ obtemos:

$$
\begin{aligned}
G_{\varepsilon}^{\prime}(t) & =\frac{\Gamma^{\prime}\left(1+i t \lambda^{-1}\right)}{\Gamma\left(1+i t \lambda^{-1}\right)} i \lambda^{-1}-i \ln \Gamma\left(1+\lambda^{-1}\right) \\
& =\frac{i}{\lambda} \Psi\left(1+i t \lambda^{-1}\right)-i \ln \Gamma\left(1+\lambda^{-1}\right) \\
G_{\varepsilon}^{(r)}(t) & =\left(\frac{i}{\lambda}\right)^{r} \Psi^{(r-1)}\left(1+i t \lambda^{-1}\right), r>1 .
\end{aligned}
$$

- Log-gama:

$$
\begin{aligned}
G_{\varepsilon}(t) & =\ln \left\{\frac{\Gamma(\lambda+i t)}{\lambda^{i t} \Gamma(\lambda)}\right\} \\
& =\ln \Gamma(\lambda+i t)-i t \ln \lambda-\ln \Gamma(\lambda) .
\end{aligned}
$$

Derivando em relação a $t$ obtemos:

$$
\begin{aligned}
G_{\varepsilon}^{\prime}(t) & =\frac{\Gamma^{\prime}(\lambda+i t)}{\Gamma(\lambda+i t)} i-i \ln \lambda \\
& =i \Psi(\lambda+i t)-i \ln \lambda . \\
G_{\varepsilon}^{(r)}(t)= & i^{r} \Psi^{(r-1)}(\lambda+i t), r>1 .
\end{aligned}
$$

Demonstração da proposição 4.12. Escrevendo recursivamente $\psi_{j+k}$ em função de $\psi_{j}$ obtemos:

$$
\begin{aligned}
& \psi_{j+1}=\delta+\phi \psi_{j}+\sigma_{\eta} \eta_{j+1} \\
\psi_{j+2}= & \delta+\phi \psi_{j+1}+\sigma_{\eta} \eta_{j+2} \\
= & \delta+\phi\left(\delta+\phi \psi_{j}+\sigma_{\eta} \eta_{j+1}\right)+\sigma_{\eta} \eta_{j+2} \\
= & \delta+\phi \delta+\phi^{2} \psi_{j}+\phi \sigma_{\eta} \eta_{j+1}+\sigma_{\eta} \eta_{j+2}
\end{aligned}
$$




$$
\begin{aligned}
\psi_{j+3}= & \delta+\phi \psi_{j+2}+\sigma_{\eta} \eta_{j+3} \\
= & \delta+\phi\left(\delta+\phi \delta+\phi^{2} \psi_{j}+\phi \sigma_{\eta} \eta_{j+1}+\sigma_{\eta} \eta_{j+2}\right)+\sigma_{\eta} \eta_{j+3} \\
= & \delta+\phi \delta+\phi^{2} \delta+\phi^{3} \psi_{j}+\phi^{2} \sigma_{\eta} \eta_{j+1}+\phi \sigma_{\eta} \eta_{j+2}+\sigma_{\eta} \eta_{j+3} \\
\vdots & \\
\psi_{j+k}= & \frac{1-\phi^{k}}{1-\phi} \delta+\phi^{k} \psi_{j}+\sigma_{\eta} \sum_{l=0}^{k-1} \phi^{l} \eta_{j+k-l}, \quad j=1, \cdots, T-k .
\end{aligned}
$$

Demonstração da proposição 4.13. Utilizando (4.32) temos que $y_{j}=\psi_{j}+\varepsilon_{j}$. Podemos então escrever a função característica do bloco como:

$$
\begin{aligned}
\varphi_{z}(r) & =E\left\{\exp \left(i r_{1} y_{j}+i r_{2} y_{j+1}+\cdots+i r_{p+1} y_{j+p}\right)\right\}= \\
& =E\left\{\exp \left(i r_{1} \psi_{j}+i r_{1} \varepsilon_{j}+i r_{2} \psi_{j+1}+i r_{2} \varepsilon_{j+1}+\cdots+i r_{p+1} \psi_{j+p}+i r_{p+1} \varepsilon_{j+p}\right)\right\} .
\end{aligned}
$$

Como os $\varepsilon_{j}$ são independentemente e identicamente distribuídos podemos escrever (C.6) como:

$$
\begin{aligned}
\varphi_{z}(r) & =E\left\{\exp \left(i r_{1} \psi_{j}+i r_{2} \psi_{j+1}+\cdots+i r_{p+1} \psi_{j+p}\right)\right\} \prod_{\zeta=1}^{p+1} E\left\{\exp \left(i r_{\zeta} \varepsilon_{j+\zeta-1}\right)\right\}= \\
& =E\left\{\exp \left(i r_{1} \psi_{j}+i r_{2} \psi_{j+1}+\cdots+i r_{p+1} \psi_{j+p}\right)\right\} \prod_{\zeta=1}^{p+1} \varphi_{\varepsilon}\left(r_{\zeta}\right) .
\end{aligned}
$$

Antes de prosseguirmos note que utilizando (4.12) podemos escrever $r_{1} \psi_{j}+\cdots+r_{p+1} \psi_{j+p}$ como:

$$
\begin{aligned}
& r_{1} \psi_{j}+\cdots+r_{p+1} \psi_{j+p}= r_{1} \psi_{j}+ \\
&+r_{2}\left\{\delta+\phi \psi_{j}+\sigma_{\eta} \eta_{j+1}\right\}+ \\
&+r_{3}\left\{\frac{1-\phi^{2}}{1-\phi} \delta+\phi^{2} \psi_{j}+\sigma_{\eta}\left[\phi \eta_{j+1}+\eta_{j+2}\right]\right\}+ \\
& \vdots \\
&+r_{p+1}\left\{\frac{1-\phi^{p}}{1-\phi} \delta+\phi^{p} \psi_{j}+\sigma_{\eta} \sum_{l=0}^{p-1} \phi^{l} \eta_{j+p-l}\right\} . \\
& r_{1} \psi_{j}+\cdots+r_{p+1} \psi_{j+p}=\frac{\delta}{1-\phi} \sum_{\zeta=2}^{p+1}\left(1-\phi^{\zeta-1}\right) r_{\zeta}+\psi_{j} \sum_{\zeta=1}^{p+1} \phi^{\zeta-1} r_{\zeta}+\sigma_{\eta} \sum_{l=2}^{p+1} \eta_{j+l-1} \sum_{\zeta=l}^{p+1} \phi^{\zeta-l} r_{\zeta} .
\end{aligned}
$$

Substituindo agora ([C.8) em (C.7), e lembrando que os $\eta_{j}$ são identicamente e independen- 
temente distribuídos temos:

$$
\begin{gathered}
\varphi_{z}(r)=E\left\{\exp \left(\frac{i \delta}{1-\phi} \sum_{\zeta=2}^{p+1}\left(1-\phi^{\zeta-1}\right) r_{\zeta}+i \psi_{j} \sum_{\zeta=1}^{p+1} \phi^{\zeta-1} r_{\zeta}+i \sigma_{\eta} \sum_{l=2}^{p+1} \eta_{j+l-1} \sum_{\zeta=l}^{p+1} \phi^{\zeta-l} r_{\zeta}\right)\right\} . \\
\cdot \prod_{\zeta=1}^{p+1} \varphi_{\varepsilon}\left(r_{\zeta}\right), \\
\varphi_{z}(r)=\exp \left(\frac{i \delta}{1-\phi} \sum_{\zeta=2}^{p+1}\left(1-\phi^{\zeta-1}\right) r_{\zeta}\right) \varphi_{\psi}\left(\sum_{\zeta=1}^{p+1} \phi^{\zeta-1} r_{\zeta}\right) . \\
\quad \prod_{j=2}^{p+1} \varphi_{\eta}\left(\sigma_{\eta} \sum_{\zeta=j}^{p+1} \phi^{\zeta-j} r_{\zeta}\right) \prod_{j=1}^{p+1} \varphi_{\varepsilon}\left(r_{j}\right) .
\end{gathered}
$$

Substituindo (4.16) e (4.15) na equação acima obtemos:

$$
\begin{gathered}
\varphi_{z}(r)=\exp \left\{\frac{i \delta}{1-\phi} \sum_{\zeta=2}^{p+1}\left(1-\phi^{\zeta-1}\right) r_{\zeta}+\frac{i \delta}{1-\phi}\left(\sum_{\zeta=1}^{p+1} \phi^{\zeta-1} r_{\zeta}\right)-\frac{\sigma_{\eta}^{2}}{2\left(1-\phi^{2}\right)}\left(\sum_{\zeta=1}^{p+1} \phi^{\zeta-1} r_{\zeta}\right)^{2}\right\} . \\
. \exp \left\{-\sum_{j=2}^{p+1} \frac{\sigma_{\eta}^{2}}{2}\left(\sum_{\zeta=j}^{p+1} \phi^{\zeta-j} r_{\zeta}\right)^{2}\right\} \prod_{j=1}^{p+1} \varphi_{\varepsilon}\left(r_{j}\right), \\
\varphi_{z}(r)=\exp \left\{\frac{i \delta}{1-\phi} \sum_{\zeta=1}^{p+1} r_{\zeta}-\frac{\sigma_{\eta}^{2}}{2\left(1-\phi^{2}\right)}\left(\sum_{\zeta=1}^{p+1} \phi^{\zeta-1} r_{\zeta}\right)^{2}-\right. \\
\left.-\sum_{j=2}^{p+1} \frac{\sigma_{\eta}^{2}}{2}\left(\sum_{\zeta=j}^{p+1} \phi^{\zeta-j} r_{\zeta}\right)^{2}\right\} \prod_{j=1}^{p+1} \varphi_{\varepsilon}\left(r_{j}\right) .
\end{gathered}
$$

Demonstração da proposição [5.2. Para o caso específico do problema de duração condicional estocástica podemos escrever a função $\Lambda_{k}(r, s)$, (B.26), como:

$$
\begin{aligned}
\Lambda_{k}(r, s)= & E\left[\exp \left(i r^{\prime} Z_{j}+i s^{\prime} Z_{j+k}\right)\right]= \\
= & E\left[\exp \left\{i\left(r_{1} Y_{j}+\cdots+r_{p+1} Y_{j+p}+s_{1} Y_{j+k}+\cdots+s_{p+1} Y_{j+p+k}\right)\right\}\right]= \\
= & E\left[\exp \left\{i\left(r_{1} \psi_{j}+\cdots+r_{p+1} \psi_{j+p}+s_{1} \psi_{j+k}+\cdots+s_{p+1} \psi_{j+p+k}\right)\right\}+\right. \\
& \left.+\exp \left\{i\left(r_{1} \varepsilon_{j}+\cdots+r_{p+1} \varepsilon_{j+p}+s_{1} \varepsilon_{j+k}+\cdots+s_{p+1} \varepsilon_{j+p+k}\right)\right\}\right]
\end{aligned}
$$


Se chamarmos de $w(r, s)$ o vetor $p+k+1 \times 1$ dado por

$$
w(r, s)=\left\{\begin{array}{cc}
\left(r_{1}, \cdots, r_{k}, r_{k+1}+s_{1}, \cdots, r_{p+1}+s_{p-k+1}, s_{p-k+2}, \cdots, s_{p+1}\right)^{\prime} & \text { se } k \leq p \\
\left(r_{1}, \cdots, r_{p+1}, 0, \cdots, 0, s_{1}, \cdots, s_{p+1}\right)^{\prime} & \text { se } k>p
\end{array}\right.
$$

e de $Z_{j}^{*}=\left(Y_{j}, \cdots, Y_{j+p+k}\right)^{\prime}$ poderemos escrever ([C.9) como

$$
\Lambda_{k}(r, s)=E\left[\exp \left(i w^{\prime} Z_{j}^{*}\right)\right]
$$

que é simplesmente a função poli-característica para vetores de tamanho $p+k+1$, calculada em $w \in \mathbb{R}^{p+k+1}$. Assim, utilizando (4.87) podemos escrever (C.10) como:

$$
\begin{aligned}
\Lambda_{k}(r, s)= & \exp \left\{\frac{i \delta}{1-\phi} \sum_{\zeta=1}^{p+k+1} w_{\zeta}-\frac{\sigma_{\eta}^{2}}{2\left(1-\phi^{2}\right)}\left(\sum_{\zeta=1}^{p+k+1} \phi^{\zeta-1} w_{\zeta}\right)^{2}-\right. \\
& \left.-\sum_{j=2}^{p+k+1} \frac{\sigma_{\eta}^{2}}{2}\left(\sum_{\zeta=j}^{p+k+1} \phi^{\zeta-j} w_{\zeta}\right)^{2}\right\} \prod_{j=1}^{p+k+1} \varphi_{\varepsilon}\left(w_{j}\right)
\end{aligned}
$$

Demonstração da proposição [5.4. Vamos agora mostrar que a seqüência $W_{n}=\Omega_{n}^{-1}$ é eficiente no sentido GMM. Como por hipótese $\Omega_{n} \stackrel{p}{\rightarrow} \Omega_{0}$, temos que $W_{0}=\Omega_{0}^{-1}$. Substituindo em (E.9), ver apêndice [E, obtemos

$$
A\left(\theta_{0}\right)=\left.\left.4 \frac{\partial V_{\theta}^{\prime}}{\partial \theta}\right|_{\theta_{0}} \Omega_{0}^{-1} \frac{\partial V_{\theta}}{\partial \theta^{\prime}}\right|_{\theta_{0}} .
$$

Substituindo agora (C.12) em (E.12) obtemos

$$
\begin{aligned}
B\left(\theta_{0}\right)^{-1} A\left(\theta_{0}\right) B\left(\theta_{0}\right)^{-1} & =\left(\left.\frac{\partial V_{\theta}^{\prime}}{\partial \theta} \Omega_{0}^{-1} \frac{\partial V_{\theta}}{\partial \theta^{\prime}}\right|_{\theta_{0}}\right)^{-1}\left(\left.\frac{\partial V_{\theta}^{\prime}}{\partial \theta} \Omega_{0}^{-1} \frac{\partial V_{\theta}}{\partial \theta^{\prime}}\right|_{\theta_{0}}\right)\left(\left.\frac{\partial V_{\theta}^{\prime}}{\partial \theta} \Omega_{0}^{-1} \frac{\partial V_{\theta}}{\partial \theta^{\prime}}\right|_{\theta_{0}}\right)^{-1} \\
& =\left(\left.\frac{\partial V_{\theta}^{\prime}}{\partial \theta} \Omega_{0}^{-1} \frac{\partial V_{\theta}}{\partial \theta^{\prime}}\right|_{\theta_{0}}\right)^{-1} .
\end{aligned}
$$

Logo, o estimador DECF com peso $W_{n}=\Omega_{n}^{-1}$ tem a seguinte distribuição assintótica

$$
\sqrt{n}\left(\widehat{\theta}_{n}-\theta_{0}\right) \stackrel{d}{\longrightarrow} N\left(0,\left(\left.\frac{\partial V_{\theta}^{\prime}}{\partial \theta} \Omega_{0}^{-1} \frac{\partial V_{\theta}}{\partial \theta^{\prime}}\right|_{\theta_{0}}\right)^{-1}\right)
$$

que coincide com o resultado obtido por (Knight e Satchell (1997)). Este estimador é eficiente (no sentido GMM), i.e., é aquele de menor variância para o estimador. Para mostrar isto, vamos utilizar o procedimento descrito em Frangakis (2005). Inicialmente façamos a seguinte definição: 
Definição C.1 Sejam $A$ e $B$ matrizes de tamanho $k \times k$. Dizemos que $A \geq B$ se e somente se a matriz $A-B$ for positiva semidefinida. Isto é, para qualquer vetor $x \in \mathbb{R}^{k}, x^{\prime}(A-B) x \geq 0$.

Com a finalidade de deixar a notação mais leve vamos chamar de $G$ a matriz $k \times 2 q$ dada por $G=\left.\frac{\partial V_{\theta}}{\partial \theta^{\prime}}\right|_{\theta_{0}}$, de $W$ a matriz $2 q \times 2 q$ dada por $W=W_{0}$ e de $\Omega$ a matriz $2 q \times 2 q$ dada por $\Omega=\Omega_{0}$. Para mostrar que o estimador DECF com peso $W_{n}=\Omega_{n}^{-1}$ é eficiente (isto é, de mínima variância), queremos mostrar que

$$
\left(G^{\prime} W G\right)^{-1} G^{\prime} W \Omega W G\left(G^{\prime} W G\right)^{-1} \geq\left(G^{\prime} \Omega^{-1} G\right)^{-1} .
$$

Vamos considerar somente o caso que a matriz $\Omega$ é de posto completo $2 q$, de modo a possuir inversa. Seja $Z$ uma variável aleatória normal $2 q$-variada com média zero e matriz de covariância $\Omega$,

$$
Z \sim N_{2 q}(0, \Omega)
$$

Note que $\left(G^{\prime} W G\right)^{-1} G^{\prime} W Z$ tem a seguinte distribuição

$$
\left(G^{\prime} W G\right)^{-1} G^{\prime} W Z \sim N_{k}\left(0,\left(G^{\prime} W G\right)^{-1} G^{\prime} W \Omega W G\left(G^{\prime} W G\right)^{-1}\right)
$$

que é a distribuição do estimador DECF. Note agora que podemos escrever

$$
\begin{aligned}
\left(G^{\prime} W G\right)^{-1} G^{\prime} W Z= & \left(G^{\prime} \Omega^{-1} G\right)^{-1} G^{\prime} \Omega^{-1} Z+ \\
& \left\{\left(G^{\prime} W G\right)^{-1} G^{\prime} W-\left(G^{\prime} \Omega^{-1} G\right)^{-1} G^{\prime} \Omega^{-1}\right\} Z .
\end{aligned}
$$

Se chamarmos $A_{1}=\left(G^{\prime} W G\right)^{-1} G^{\prime} W$ e $A_{0}=\left(G^{\prime} \Omega^{-1} G\right)^{-1} G^{\prime} \Omega^{-1}$ então podemos escrever (C.16) como

$$
A_{1} Z=A_{0} Z+\left(A_{1}-A_{0}\right) Z
$$

Podemos calcular a variância de $A_{1} Z$ por

$$
\operatorname{var}\left(A_{1} Z\right)=\operatorname{var}\left[A_{0} Z\right]+\operatorname{var}\left[\left(A_{1}-A_{0}\right) Z\right]+A_{0} \operatorname{var}[Z]\left(A_{1}-A_{0}\right)^{\prime}+\left(A_{1}-A_{0}\right) \operatorname{var}[Z] A_{0}^{\prime} .
$$

Mas

$$
\begin{aligned}
& \operatorname{var}\left[A_{0} Z\right]=A_{0} \Omega A_{0}^{\prime} \\
&=\left(G^{\prime} \Omega^{-1} G\right)^{-1} G^{\prime} \Omega G\left(G^{\prime} \Omega^{-1} G\right)^{-1} \\
&=\left(G^{\prime} \Omega^{-1} G\right)^{-1} \\
& \operatorname{var}\left[\left(A_{1}-A_{0}\right) Z\right]=\left(A_{1}-A_{0}\right) \Omega\left(A_{1}-A_{0}\right)^{\prime}
\end{aligned}
$$




$$
\begin{aligned}
A_{0} \operatorname{var}[Z]\left(A_{1}-A_{0}\right)^{\prime} & =A_{0} \Omega\left(A_{1}-A_{0}\right)^{\prime} \\
& =\left\{\left(G^{\prime} \Omega^{-1} G\right)^{-1} G^{\prime} \Omega^{-1}\right\} \Omega\left\{\left(G^{\prime} W G\right)^{-1} G^{\prime} W-\left(G^{\prime} \Omega^{-1} G\right)^{-1} G^{\prime} \Omega^{-1}\right\}^{\prime} \\
& =\left(G^{\prime} \Omega^{-1} G\right)^{-1} G^{\prime}\left\{\left(G^{\prime} W G\right)^{-1} G^{\prime} W-\left(G^{\prime} \Omega^{-1} G\right)^{-1} G^{\prime} \Omega^{-1}\right\}^{\prime} \\
& =\left(G^{\prime} \Omega^{-1} G\right)^{-1} G^{\prime} W G\left(G^{\prime} W G\right)^{-1}-\left(G^{\prime} \Omega^{-1} G\right)^{-1} G^{\prime} \Omega^{-1} G\left(G^{\prime} \Omega^{-1} G\right)^{-1} \\
& =\left(G^{\prime} \Omega^{-1} G\right)^{-1}-\left(G^{\prime} \Omega^{-1} G\right)^{-1} \\
& =0
\end{aligned}
$$

Como $\left(A_{1}-A_{0}\right) \operatorname{var}[Z] A_{0}^{\prime}=\left[A_{0} \operatorname{var}[Z]\left(A_{1}-A_{0}\right)^{\prime}\right]^{\prime}=0$, se substituirmos (C.21) em (C.18) obtemos:

$$
A_{1} \Omega A_{1}^{\prime}=A_{0} \Omega A_{0}^{\prime}+\left(A_{1}-A_{0}\right) \Omega\left(A_{1}-A_{0}\right)^{\prime} .
$$

Seja agora $x \in \mathbb{R}^{k}$. Se pré-multiplicarmos ambos os lados da equação acima por $x^{\prime}$ e pósmultiplicarmos por $x$ obteremos

$$
x^{\prime} A_{1} \Omega A_{1}^{\prime} x=x^{\prime} A_{0} \Omega A_{0}^{\prime} x+x^{\prime}\left(A_{1}-A_{0}\right) \Omega\left(A_{1}-A_{0}\right)^{\prime} x .
$$

Chamando agora $x_{1}=A_{1}^{\prime} x, x_{0}=A_{0}^{\prime} x$ e $x_{10}=\left(A_{1}-A_{0}\right)^{\prime} x$ podemos reescrever a equação acima como

$$
x_{1}^{\prime} \Omega x_{1}=x_{0}^{\prime} \Omega x_{0}+x_{10}^{\prime} \Omega x_{10} .
$$

Lembrando que $\Omega$ é positiva definida, temos que $x_{1}^{\prime} \Omega x_{1} \geq 0, x_{0}^{\prime} \Omega x_{0} \geq 0$ e $x_{10}^{\prime} \Omega x_{10} \geq 0$. Logo, $x_{1}^{\prime} \Omega x_{1} \geq x_{0}^{\prime} \Omega x_{0}$, ou ainda, $x^{\prime} A_{1} \Omega A_{1}^{\prime} x \geq x^{\prime} A_{0} \Omega A_{0}^{\prime} x$, o que implica em $A_{1} \Omega A_{1}^{\prime} \geq A_{0} \Omega A_{0}^{\prime}$, ou

$$
\left(G^{\prime} W G\right)^{-1} G^{\prime} W \Omega W G\left(G^{\prime} W G\right)^{-1} \geq\left(G^{\prime} \Omega^{-1} G\right)^{-},
$$

que era o que queriamos demonstrar.

Demonstração da proposição 5.5. As derivadas da função característica dos log-erros, $\varphi_{\varepsilon}(t)$, em relação aos parâmetros do modelo SCD são dadas por:

\section{- Exponencial:}

$$
\begin{gathered}
\frac{d \varphi_{\varepsilon}(t)}{d r}=\varphi_{\varepsilon}^{\prime}(t)=i \Gamma^{\prime}(1+i t)=i \Gamma(1+i t) \Psi(1+i t) \\
\frac{\partial \varphi_{\varepsilon}(t)}{\partial \lambda}=0
\end{gathered}
$$


- Weibull:

$$
\begin{aligned}
& \frac{d \varphi_{\varepsilon}(t)}{d t}=\varphi_{\varepsilon}^{\prime}(t)=\frac{\Gamma^{\prime}\left(1+i t \lambda^{-1}\right) i \lambda^{-1}}{\left[\Gamma\left(1+\lambda^{-1}\right)\right]^{i t}}+\frac{\Gamma\left(1+i t \lambda^{-1}\right)(-i) \ln \Gamma\left(1+\lambda^{-1}\right)}{\left[\Gamma\left(1+\lambda^{-1}\right)\right]^{i t}} \\
& =i \frac{\left[\lambda^{-1} \Gamma^{\prime}\left(1+i t \lambda^{-1}\right)-\Gamma\left(1+i t \lambda^{-1}\right) \ln \Gamma\left(1+\lambda^{-1}\right)\right]}{\left[\Gamma\left(1+\lambda^{-1}\right)\right]^{i t}} \\
& =i \frac{\Gamma\left(1+i t \lambda^{-1}\right)}{\left[\Gamma\left(1+\lambda^{-1}\right)\right]^{i t}}\left\{\frac{1}{\lambda} \frac{\Gamma^{\prime}\left(1+i t \lambda^{-1}\right)}{\Gamma\left(1+i t \lambda^{-1}\right)}-\ln \Gamma\left(1+\lambda^{-1}\right)\right\} \\
& \varphi_{\varepsilon}^{\prime}(t)=i \frac{\Gamma\left(1+i t \lambda^{-1}\right)}{\left[\Gamma\left(1+\lambda^{-1}\right)\right]^{i t}}\left\{\frac{1}{\lambda} \Psi\left(1+i t \lambda^{-1}\right)-\ln \Gamma\left(1+\lambda^{-1}\right)\right\} \\
& \frac{\partial \varphi_{\varepsilon}(t)}{\partial \lambda}=\frac{\Gamma^{\prime}\left(1+i t \lambda^{-1}\right) i t(-1) \lambda^{-2}}{\left[\Gamma\left(1+\lambda^{-1}\right)\right]^{i t}}+\frac{\Gamma\left(1+i t \lambda^{-1}\right)(-i t) \Gamma^{\prime}\left(1+\lambda^{-1}\right)\left(-\lambda^{-2}\right)}{\left[\Gamma\left(1+\lambda^{-1}\right)\right]^{i t+1}}= \\
& =\frac{i t \Gamma\left(1+i t \lambda^{-1}\right)}{\lambda^{2}\left[\Gamma\left(1+\lambda^{-1}\right)\right]^{i t}}\left\{-\frac{\Gamma^{\prime}\left(1+i t \lambda^{-1}\right)}{\Gamma\left(1+i t \lambda^{-1}\right)}+\frac{\Gamma^{\prime}\left(1+\lambda^{-1}\right)}{\Gamma\left(1+\lambda^{-1}\right)}\right\} \\
& \frac{\partial \varphi_{\varepsilon}(t)}{\partial \lambda}=\frac{i t}{\lambda^{2}} \frac{\Gamma\left(1+i t \lambda^{-1}\right)}{\left[\Gamma\left(1+\lambda^{-1}\right)\right]^{i t}}\left\{-\Psi\left(1+i t \lambda^{-1}\right)+\Psi\left(1+\lambda^{-1}\right)\right\}
\end{aligned}
$$

- Gama:

$$
\begin{gathered}
\frac{d \varphi_{\varepsilon}(t)}{d t}=\varphi_{\varepsilon}^{\prime}(t)=\frac{\Gamma^{\prime}(\lambda+i t) i}{\lambda^{i t} \Gamma(\lambda)}+\frac{\Gamma(\lambda+i t)(-i) \ln \lambda}{\lambda^{i t} \Gamma(\lambda)} \\
=i \frac{\left[\Gamma^{\prime}(\lambda+i t)-\Gamma(\lambda+i t) \ln \lambda\right]}{\lambda^{i t} \Gamma(\lambda)} \\
=\frac{i}{\lambda^{i t}} \frac{\Gamma(\lambda+i t)}{\Gamma(\lambda)}\left\{\frac{\Gamma^{\prime}(\lambda+i t)}{\Gamma(\lambda+i t)}-\ln \lambda\right\} \\
\varphi_{\varepsilon}^{\prime}(t)=\frac{i}{\lambda^{i t}} \frac{\Gamma(\lambda+i t)}{\Gamma(\lambda)}\{\Psi(\lambda+i t)-\ln \lambda\} \\
\frac{\partial \varphi_{\varepsilon}(t)}{\partial \lambda}=\frac{\Gamma^{\prime}(\lambda+i t)}{\lambda^{i t} \Gamma(\lambda)}+\frac{\Gamma(\lambda+i t)(-i t)}{\lambda^{i t+1} \Gamma(\lambda)}+\frac{\Gamma(\lambda+i t)(-1) \Gamma^{\prime}(\lambda)}{\lambda^{i t}[\Gamma(\lambda)]^{2}} \\
=\frac{\Gamma(\lambda+i t)}{\lambda^{i t} \Gamma(\lambda)}\left\{\frac{\Gamma^{\prime}(\lambda+i t)}{\Gamma(\lambda+i t)}-\frac{i t}{\lambda}-\frac{\Gamma^{\prime}(\lambda)}{\Gamma(\lambda)}\right\} \\
\frac{\partial \varphi_{\varepsilon}(t)}{\partial \lambda}=\frac{1}{\lambda^{i t}} \frac{\Gamma(\lambda+i t)}{\Gamma(\lambda)}\left\{\Psi(\lambda+i t)-\frac{i t}{\lambda}-\Psi(\lambda)\right\}
\end{gathered}
$$

Demonstração da proposição 5.6. As derivadas da função poli-característica, $\varphi_{z}(r)$, em 
relação aos parâmetros do modelo SCD são dadas por:

$$
\begin{gathered}
\frac{\partial \varphi_{z}(r)}{\partial \delta}=\left(\frac{i}{1-\phi} \sum_{\zeta=1}^{p+1} r_{\zeta}\right) \exp \left\{\frac{i \delta}{1-\phi} \sum_{\zeta=1}^{p+1} r_{\zeta}-\frac{\sigma_{\eta}^{2}}{2\left(1-\phi^{2}\right)}\left(\sum_{\zeta=1}^{p+1} \phi^{\zeta-1} r_{\zeta}\right)^{2}-\right. \\
\left.-\sum_{j=2}^{p+1} \frac{\sigma_{\eta}^{2}}{2}\left(\sum_{\zeta=j}^{p+1} \phi^{\zeta-j} r_{\zeta}\right)^{2}\right\} \prod_{j=1}^{p+1} \varphi_{\varepsilon}\left(r_{j}\right) \\
\frac{\partial \varphi_{z}(r)}{\partial \delta}=\varphi_{z}(r)\left(\frac{i}{1-\phi} \sum_{\zeta=1}^{p+1} r_{\zeta}\right)
\end{gathered}
$$

$$
\begin{aligned}
\frac{\partial \varphi_{z}(r)}{\partial \phi}= & \exp \left\{\frac{i \delta}{1-\phi} \sum_{\zeta=1}^{p+1} r_{\zeta}-\frac{\sigma_{\eta}^{2}}{2\left(1-\phi^{2}\right)}\left(\sum_{\zeta=1}^{p+1} \phi^{\zeta-1} r_{\zeta}\right)^{2}-\sum_{j=2}^{p+1} \frac{\sigma_{\eta}^{2}}{2}\left(\sum_{\zeta=j}^{p+1} \phi^{\zeta-j} r_{\zeta}\right)^{2}\right\} . \\
& \cdot \prod_{j=1}^{p+1} \varphi_{\varepsilon}\left(r_{j}\right) \cdot\left\{\frac{i \delta}{(1-\phi)^{2}} \sum_{\zeta=1}^{p+1} r_{\zeta}-\frac{\sigma_{\eta}^{2} \phi}{\left(1-\phi^{2}\right)^{2}}\left(\sum_{\zeta=1}^{p+1} \phi^{\zeta-1} r_{\zeta}\right)^{2}-\right. \\
& -\frac{\sigma_{\eta}^{2}}{\left(1-\phi^{2}\right)}\left(\sum_{\zeta=1}^{p+1} \phi^{\zeta-1} r_{\zeta}\right)\left(\sum_{\zeta=1}^{p+1}(\zeta-1) \phi^{\zeta-2} r_{\zeta}\right)- \\
& \left.-\sum_{j=2}^{p+1} \sigma_{\eta}^{2}\left(\sum_{\zeta=j}^{p+1} \phi^{\zeta-j} r_{\zeta}\right)\left(\sum_{\zeta=j}^{p+1}(\zeta-j) \phi^{\zeta-j-1} r_{\zeta}\right)\right\}
\end{aligned}
$$

$$
\begin{aligned}
\frac{\partial \varphi_{z}(r)}{\partial \phi}= & \varphi_{z}(r)\left\{\frac{i \delta}{(1-\phi)^{2}} \sum_{\zeta=1}^{p+1} r_{\zeta}-\frac{\sigma_{\eta}^{2} \phi}{\left(1-\phi^{2}\right)^{2}}\left(\sum_{\zeta=1}^{p+1} \phi^{\zeta-1} r_{\zeta}\right)^{2}-\right. \\
& -\frac{\sigma_{\eta}^{2}}{\left(1-\phi^{2}\right)}\left(\sum_{\zeta=1}^{p+1} \phi^{\zeta-1} r_{\zeta}\right)\left(\sum_{\zeta=1}^{p+1}(\zeta-1) \phi^{\zeta-2} r_{\zeta}\right)- \\
& \left.-\sum_{j=2}^{p+1} \sigma_{\eta}^{2}\left(\sum_{\zeta=j}^{p+1} \phi^{\zeta-j} r_{\zeta}\right)\left(\sum_{\zeta=j}^{p+1}(\zeta-j) \phi^{\zeta-j-1} r_{\zeta}\right)\right\}
\end{aligned}
$$


149

$$
\begin{aligned}
& \frac{\partial \varphi_{z}(r)}{\partial \sigma_{\eta}}=\exp \left\{\frac{i \delta}{1-\phi} \sum_{\zeta=1}^{p+1} r_{\zeta}-\frac{\sigma_{\eta}^{2}}{2\left(1-\phi^{2}\right)}\left(\sum_{\zeta=1}^{p+1} \phi^{\zeta-1} r_{\zeta}\right)^{2}-\right. \\
& \left.-\sum_{j=2}^{p+1} \frac{\sigma_{\eta}^{2}}{2}\left(\sum_{\zeta=j}^{p+1} \phi^{\zeta-j} r_{\zeta}\right)^{2}\right\} \prod_{j=1}^{p+1} \varphi_{\varepsilon}\left(r_{j}\right) \text {. } \\
& \left\{-\frac{2 \sigma_{\eta}}{2\left(1-\phi^{2}\right)}\left(\sum_{\zeta=1}^{p+1} \phi^{\zeta-1} r_{\zeta}\right)^{2}-\sum_{j=2}^{p+1} \frac{2 \sigma_{\eta}}{2}\left(\sum_{\zeta=j}^{p+1} \phi^{\zeta-j} r_{\zeta}\right)^{2}\right\} \\
& \frac{\partial \varphi_{z}(r)}{\partial \sigma_{\eta}}=\varphi_{z}(r)\left\{-\frac{\sigma_{\eta}}{\left(1-\phi^{2}\right)}\left(\sum_{\zeta=1}^{p+1} \phi^{\zeta-1} r_{\zeta}\right)^{2}-\sum_{j=2}^{p+1} \sigma_{\eta}\left(\sum_{\zeta=j}^{p+1} \phi^{\zeta-j} r_{\zeta}\right)^{2}\right\} \\
& \frac{\partial \varphi_{z}(r)}{\partial \lambda}=\exp \left\{\frac{i \delta}{1-\phi} \sum_{\zeta=1}^{p+1} r_{\zeta}-\frac{\sigma_{\eta}^{2}}{2\left(1-\phi^{2}\right)}\left(\sum_{\zeta=1}^{p+1} \phi^{\zeta-1} r_{\zeta}\right)^{2}-\right. \\
& \left.-\sum_{j=2}^{p+1} \frac{\sigma_{\eta}^{2}}{2}\left(\sum_{\zeta=j}^{p+1} \phi^{\zeta-j} r_{\zeta}\right)^{2}\right\}\left\{\sum_{j=1}^{p+1} \frac{\partial \varphi_{\varepsilon}\left(r_{j}\right)}{\partial \lambda} \prod_{\substack{k=1 \\
k \neq j}}^{p+1} \varphi_{\varepsilon}\left(r_{k}\right)\right\} \\
& \frac{\partial \varphi_{z}(r)}{\partial \lambda}=\varphi_{z}(r) \sum_{j=1}^{p+1} \frac{1}{\varphi_{\varepsilon}\left(r_{j}\right)} \frac{\partial \varphi_{\varepsilon}\left(r_{j}\right)}{\partial \lambda}
\end{aligned}
$$





\section{Apêndice D}

\section{Matriz de covariância assintótica do estimador CECF}

Neste apêndice vamos obter a matriz de covariância assintótica do estimador CECF. Como o estimador CECF pertence à classe dos estimadores extremos podemos, a princípio, tentar aplicar o teorema 4.1.3 de Amemiya (1985) apresentado no apêndice A. A fim de deixar a notação um pouco mais leve vamos usar as seguintes variáveis:

$$
\begin{aligned}
& u_{n}(r)=\operatorname{Re} \varphi_{n}(r)=\frac{1}{n} \sum_{j=1}^{n} \cos \left(r^{\prime} Z_{j}\right), \\
& v_{n}(r)=\operatorname{Im} \varphi_{n}(r)=\frac{1}{n} \sum_{j=1}^{n} \sin \left(r^{\prime} Z_{j}\right) .
\end{aligned}
$$

Lembremos, também, que a função característica pode ser escrita como:

$$
\begin{aligned}
\varphi_{z}(r) & =E\left[\exp \left(i r^{\prime} Z_{j}\right)\right]= \\
& =E\left[\cos \left(r^{\prime} Z_{j}\right)+i \sin \left(r^{\prime} Z_{j}\right)\right]= \\
& =\operatorname{Re} \varphi_{z}(r)+i \operatorname{Im} \varphi_{z}(r) \\
& =u(r ; \theta)+i v(r ; \theta),
\end{aligned}
$$

em que explicitamos a dependência das funções $u$ e $v$ do vetor de parâmetros $\theta$ :

$$
\begin{aligned}
& u(r ; \theta)=\operatorname{Re} \varphi_{z}(r)=E\left[\cos \left(r^{\prime} Z_{j}\right)\right] \\
& v(r ; \theta)=\operatorname{Im} \varphi_{z}(r)=E\left[\sin \left(r^{\prime} Z_{j}\right)\right] .
\end{aligned}
$$

Como o estimador CECF é obtido a partir da minimização da função de distância $Q_{n}$, precisamos obter a primeira e a segunda derivadas em relação ao vetor de parâmetros $\theta$, a 
fim de aplicarmos o citado teorema de Amemiva (1985). Assim vamos escrever $Q_{n}$ como

$$
\begin{aligned}
Q_{n}(\theta) & =\int\left|\varphi_{n}(r)-\varphi(r ; \theta)\right|^{2} d G(r) \\
& =\int\left|u_{n}(r)+i v_{n}(r)-u(r ; \theta)-i v(r ; \theta)\right|^{2} d G(r) \\
& =\int\left|\left(u_{n}(r)-u(r ; \theta)\right)+i\left(v_{n}(r)-v(r ; \theta)\right)\right|^{2} d G(r) \\
& =\int\left[\left(u_{n}(r)-u(r ; \theta)\right)+i\left(v_{n}(r)-v(r ; \theta)\right)\right] \overline{\left[\left(u_{n}(r)-u(r ; \theta)\right)+i\left(v_{n}(r)-v(r ; \theta)\right)\right]} d G(r) \\
& =\int\left[\left(u_{n}(r)-u(r ; \theta)\right)+i\left(v_{n}(r)-v(r ; \theta)\right)\right]\left[\left(u_{n}(r)-u(r ; \theta)\right)-i\left(v_{n}(r)-v(r ; \theta)\right)\right] d G(r) \\
& =\int\left[\left(u_{n}(r)-u(r ; \theta)\right)^{2}+\left(v_{n}(r)-v(r ; \theta)\right)^{2}\right] d G(r) .
\end{aligned}
$$

Assumindo as condições usuais de regularidade para que possamos derivar sob o sinal de integração e derivando (D.4) em relação a $\theta$ obtemos

$$
\frac{\partial Q_{n}}{\partial \theta}=-2 \int\left\{\left(u_{n}(r)-u(r ; \theta)\right) \frac{\partial u(r ; \theta)}{\partial \theta}+\left(v_{n}(r)-v(r ; \theta)\right) \frac{\partial v(r ; \theta)}{\partial \theta}\right\} d G(r)
$$

Como $E\left[u_{n}(r)\right]=u(r ; \theta)$ e $E\left[v_{n}(r)\right]=v(r ; \theta)$ temos que $E\left[\frac{\partial Q_{n}}{\partial \theta}\right]=0$. Agora, derivando (D.5) em relação a $\theta$ obtemos:

$$
\begin{aligned}
\frac{\partial^{2} Q_{n}}{\partial \theta \partial \theta^{\prime}}= & -2 \int\left\{(-1) \frac{\partial u(r ; \theta)}{\partial \theta} \frac{\partial u(r ; \theta)}{\partial \theta^{\prime}}+\left(u_{n}(r)-u(r ; \theta)\right) \frac{\partial^{2} u(r ; \theta)}{\partial \theta \partial \theta^{\prime}}+\right. \\
& \left.+(-1) \frac{\partial v(r ; \theta)}{\partial \theta} \frac{\partial v(r ; \theta)}{\partial \theta^{\prime}}+\left(v_{n}(r)-v(r ; \theta)\right) \frac{\partial^{2} v(r ; \theta)}{\partial \theta \partial \theta^{\prime}}\right\} d G(r) \\
= & 2 \int\left\{\frac{\partial u(r ; \theta)}{\partial \theta} \frac{\partial u(r ; \theta)}{\partial \theta^{\prime}}+\frac{\partial v(r ; \theta)}{\partial \theta} \frac{\partial v(r ; \theta)}{\partial \theta^{\prime}}\right\} d G(r)- \\
& -2 \int\left\{\left(u_{n}(r)-u(r ; \theta)\right) \frac{\partial^{2} u(r ; \theta)}{\partial \theta \partial \theta^{\prime}}+\left(v_{n}(r)-v(r ; \theta)\right) \frac{\partial^{2} v(r ; \theta)}{\partial \theta \partial \theta^{\prime}}\right\} d G(r) .
\end{aligned}
$$

Note agora que

$$
\begin{aligned}
E\left[\frac{1}{n} \frac{\partial^{2} Q_{n}}{\partial \theta \partial \theta^{\prime}}\right] & =\frac{1}{n} E\left[\frac{\partial^{2} Q_{n}}{\partial \theta \partial \theta^{\prime}}\right] \\
& =\frac{2}{n} \int\left\{\frac{\partial u(r ; \theta)}{\partial \theta} \frac{\partial u(r ; \theta)}{\partial \theta^{\prime}}+\frac{\partial v(r ; \theta)}{\partial \theta} \frac{\partial v(r ; \theta)}{\partial \theta^{\prime}}\right\} d G(r) .
\end{aligned}
$$

Se $\int\left\{\frac{\partial u(r ; \theta)}{\partial \theta} \frac{\partial u(r ; \theta)}{\partial \theta^{\prime}}+\frac{\partial v(r ; \theta)}{\partial \theta} \frac{\partial v(r ; \theta)}{\partial \theta^{\prime}}\right\} d G(r)<\infty$, temos que

$$
\widetilde{B}\left(\theta_{0}\right)=\lim _{n \rightarrow \infty} E\left[\left.\frac{1}{n} \frac{\partial^{2} Q_{n}}{\partial \theta \partial \theta^{\prime}}\right|_{\theta_{0}}\right]=0,
$$

i.e., $\widetilde{B}\left(\theta_{0}\right)$ é uma matriz singular, e, portanto, a condição (2) do teorema 4.1.3 de Amemiya 
(1985) não está satisfeita. Para resolver esta dificuldade vamos modificar a equação (A.4) na demonstração do referido teorema, da seguinte maneira:

$$
\sqrt{n}\left(\widehat{\theta}_{n}-\theta_{0}\right)=-\left[\left.\frac{\partial^{2} Q_{n}}{\partial \theta \partial \theta^{\prime}}\right|_{\theta^{*}}\right]^{-1}\left(\left.\sqrt{n} \frac{\partial Q_{n}}{\partial \theta}\right|_{\theta_{0}}\right) .
$$

Isto é, vamos admitir que $\left(\partial^{2} Q_{n} / \partial \theta \partial \theta^{\prime}\right)_{\theta_{n}^{*}}$ convirja em probabilidade para uma matriz não singular $B\left(\theta_{0}\right)=\lim _{n \rightarrow \infty} E\left[\left(\partial^{2} Q_{n} / \partial \theta \partial \theta^{\prime}\right)_{\theta_{0}}\right]$ para toda seqüência $\theta_{n}^{*}$ tal que $\theta_{n}^{*} \stackrel{\text { a.s. }}{\longrightarrow} \theta_{0}$ e que $n^{1 / 2}\left(\partial Q_{n} / \partial \theta\right)_{\theta_{0}} \stackrel{d}{\longrightarrow} N\left(0, A\left(\theta_{0}\right)\right)$, sendo $A\left(\theta_{0}\right)=\lim _{n \rightarrow \infty} E\left[n\left(\partial Q_{n} / \partial \theta\right)_{\theta_{0}} \cdot\left(\partial Q_{n} / \partial \theta^{\prime}\right)_{\theta_{0}}\right]$, obtendo, assim, o mesmo resultado que o teorema 4.1.3 de Amemiva (1985).

Antes de prosseguirmos vamos reescrever (D.5) como

$$
\begin{aligned}
\frac{\partial Q_{n}}{\partial \theta}= & -2 \int\left\{\left(\frac{1}{n} \sum_{j=1}^{n} \cos \left(r^{\prime} Z_{j}\right)-u(r ; \theta)\right) \frac{\partial u(r ; \theta)}{\partial \theta}+\right. \\
& \left.+\left(\frac{1}{n} \sum_{j=1}^{n} \sin \left(r^{\prime} Z_{j}\right)-v(r ; \theta)\right) \frac{\partial v(r ; \theta)}{\partial \theta}\right\} d G(r) \\
= & -\frac{2}{n} \sum_{j=1}^{n} \int\left\{\left(\cos \left(r^{\prime} Z_{j}\right)-u(r ; \theta) \frac{\partial u(r ; \theta)}{\partial \theta}+\left(\sin \left(r^{\prime} Z_{j}\right)-v(r ; \theta)\right) \frac{\partial v(r ; \theta)}{\partial \theta}\right\} d G(r)\right. \\
= & -\frac{2}{n} \sum_{j=1}^{n} K_{j}(\theta),
\end{aligned}
$$

em que

$K_{j}(\theta)=\int\left\{\left(\cos \left(r^{\prime} Z_{j}\right)-\operatorname{Re} \varphi(r ; \theta)\right) \frac{\partial \operatorname{Re} \varphi(r ; \theta)}{\partial \theta}+\left(\sin \left(r^{\prime} Z_{j}\right)-\operatorname{Im} \varphi(r ; \theta)\right) \frac{\partial \operatorname{Im} \varphi(r ; \theta)}{\partial \theta}\right\} d G(r)$.

Vamos agora mostrar que

$$
\left.\sqrt{n} \frac{\partial Q_{n}}{\partial \theta}\right|_{\theta_{0}} \stackrel{d}{\rightarrow} N\left(0, A\left(\theta_{0}\right)\right)
$$


Lembrando que $E\left[\left(\partial Q_{n} / \partial \theta\right)_{\theta_{0}}\right]=0$ temos que

$$
\begin{aligned}
\operatorname{var}\left[\sqrt{n} \frac{\partial Q_{n}}{\partial \theta}\right] & =n E\left[\frac{\partial Q_{n}}{\partial \theta} \frac{\partial Q_{n}}{\partial \theta^{\prime}}\right] \\
& =n E\left[\left(-\frac{2}{n} \sum_{j=1}^{n} K_{j}(\theta)\right)\left(-\frac{2}{n} \sum_{k=1}^{n} K_{k}(\theta)^{\prime}\right)\right] \\
& =\frac{4}{n} E\left[\sum_{j=1}^{n} \sum_{k=1}^{n} K_{j}(\theta) K_{k}(\theta)^{\prime}\right] \\
& =\frac{4}{n} E\left[\sum_{j=1}^{n} K_{j}(\theta) K_{j}(\theta)^{\prime}+\sum_{k=1}^{n-1}(n-k) K_{1}(\theta) K_{1+k}(\theta)^{\prime}+\sum_{k=1}^{n-1}(n-k) K_{1+k}(\theta) K_{1}(\theta)^{\prime}\right] \\
& =4\left\{E\left[K_{1}(\theta) K_{1}(\theta)^{\prime}\right]+\frac{1}{n} \sum_{k=1}^{n}(n-k)\left(E\left[K_{1}(\theta) K_{1+k}(\theta)^{\prime}\right]+E\left[K_{1+k}(\theta) K_{1}(\theta)^{\prime}\right]\right)\right\} .
\end{aligned}
$$

Relembremos que

$$
\begin{aligned}
\left.\sqrt{n} \frac{\partial Q_{n}}{\partial \theta}\right|_{\theta_{0}} & =\sqrt{n}\left[-\frac{2}{n} \sum_{j=1}^{n} K_{j}\left(\theta_{0}\right)\right] \\
& =\frac{1}{\sqrt{n}}\left[-2 \sum_{j=1}^{n} K_{j}\left(\theta_{0}\right)\right] .
\end{aligned}
$$

Note que (D.10) é uma seqüência de somas de variáveis dependentes (os $\left.K_{j}\right)$, limitadas (já que são integrais de senos e cossenos). Se assumirmos a condição de $\alpha$-mixing para a seqüência $\left\{Z_{j}\right\}$, poderemos aplicar o teorema 18.5.4 de Ibragimov e Linnik (1971), obtendo $A\left(\theta_{0}\right)<\infty$, em que

$$
A\left(\theta_{0}\right)=4\left\{E\left[K_{1}\left(\theta_{0}\right) K_{1}\left(\theta_{0}\right)^{\prime}\right]+\sum_{k=1}^{\infty}\left(E\left[K_{1}\left(\theta_{0}\right) K_{1+k}\left(\theta_{0}\right)^{\prime}\right]+E\left[K_{1+k}\left(\theta_{0}\right) K_{1}\left(\theta_{0}\right)^{\prime}\right]\right)\right\},
$$

e se $A\left(\theta_{0}\right) \neq 0$,

$$
\frac{1}{\sqrt{n}}\left[-2 \sum_{j=1}^{n} K_{j}\left(\theta_{0}\right)\right]=\left.\sqrt{n} \frac{\partial Q_{n}}{\partial \theta}\right|_{\theta_{0}} \stackrel{d}{\rightarrow} N\left(0, A\left(\theta_{0}\right)\right) .
$$

Finalmente, se chamarmos

$$
\begin{aligned}
B\left(\theta_{0}\right) & =\lim _{n \rightarrow \infty} E\left[\left.\frac{\partial^{2} Q_{n}}{\partial \theta \partial \theta^{\prime}}\right|_{\theta_{0}}\right] \\
& =2 \int\left\{\frac{\partial u(r ; \theta)}{\partial \theta} \frac{\partial u(r ; \theta)}{\partial \theta^{\prime}}+\frac{\partial v(r ; \theta)}{\partial \theta} \frac{\partial v(r ; \theta)}{\partial \theta^{\prime}}\right\} d G(r)
\end{aligned}
$$


e usando (D.6) obtemos a distribuição limite do estimador CECF $\widehat{\theta}_{n}$ dada por

$$
\sqrt{n}\left(\widehat{\theta}_{n}-\theta_{0}\right) \stackrel{d}{\longrightarrow} N\left(0, B\left(\theta_{0}\right)^{-1} A\left(\theta_{0}\right) B\left(\theta_{0}\right)^{-1}\right) .
$$

Vamos agora obter uma expressão mais explícita para $A\left(\theta_{0}\right)$ dada em (D.11). Substituindo (D.8) em (D.9) obtemos

$$
\operatorname{var}\left[\sqrt{n} \frac{\partial Q_{n}}{\partial \theta}\right]=
$$

$$
\begin{aligned}
= & \frac{4}{n} E\left[\sum_{j=1}^{n} \sum_{k=1}^{n} K_{j}(\theta) K_{k}(\theta)^{\prime}\right] \\
= & \frac{4}{n} E\left[\sum_{j=1}^{n} \sum_{k=1}^{n} \int\left\{\left(\cos \left(r^{\prime} Z_{j}\right)-u(r ; \theta)\right) \frac{\partial u(r ; \theta)}{\partial \theta}+\left(\sin \left(r^{\prime} Z_{j}\right)-v(r ; \theta)\right) \frac{\partial v(r ; \theta)}{\partial \theta}\right\} d G(r) .\right. \\
& \left.\cdot \int\left\{\left(\cos \left(s^{\prime} Z_{j}\right)-u(s ; \theta)\right) \frac{\partial u(s ; \theta)}{\partial \theta^{\prime}}+\left(\sin \left(s^{\prime} Z_{j}\right)-v(s ; \theta)\right) \frac{\partial v(s ; \theta)}{\partial \theta^{\prime}}\right\} d G(s)\right] . \\
= & \frac{4}{n} E\left[\sum _ { j = 1 } ^ { n } \sum _ { k = 1 } ^ { n } \left\{\iint\left(\cos \left(r^{\prime} Z_{j}\right)-u(r ; \theta)\right)\left(\cos \left(s^{\prime} Z_{k}\right)-u(s ; \theta)\right) \frac{\partial u(r ; \theta)}{\partial \theta} \frac{\partial u(s ; \theta)}{\partial \theta^{\prime}} d G(r) d G(s)+\right.\right. \\
& +\iint\left(\cos \left(r^{\prime} Z_{j}\right)-u(r ; \theta)\right)\left(\sin \left(s^{\prime} Z_{k}\right)-v(s ; \theta)\right) \frac{\partial u(r ; \theta)}{\partial \theta} \frac{\partial v(s ; \theta)}{\partial \theta^{\prime}} d G(r) d G(s)+ \\
& +\iint\left(\sin \left(r^{\prime} Z_{j}\right)-v(r ; \theta)\right)\left(\cos \left(s^{\prime} Z_{k}\right)-u(s ; \theta)\right) \frac{\partial v(r ; \theta)}{\partial \theta} \frac{\partial u(s ; \theta)}{\partial \theta^{\prime}} d G(r) d G(s)+ \\
& \left.\left.+\iint\left(\sin \left(r^{\prime} Z_{j}\right)-v(r ; \theta)\right)\left(\sin \left(s^{\prime} Z_{k}\right)-v(s ; \theta)\right) \frac{\partial v(r ; \theta)}{\partial \theta} \frac{\partial v(s ; \theta)}{\partial \theta^{\prime}} d G(r) d G(s)\right\}\right]
\end{aligned}
$$

Ou seja,

$$
\begin{aligned}
\operatorname{var}\left[\sqrt{n} \frac{\partial Q_{n}}{\partial \theta}\right]= & 4 \iint\left\{\Omega_{R R, n}(r, s) \frac{\partial \operatorname{Re} \varphi(r ; \theta)}{\partial \theta} \frac{\partial \operatorname{Re} \varphi(s ; \theta)}{\partial \theta^{\prime}}+\right. \\
& +\Omega_{R I, n}(r, s) \frac{\partial \operatorname{Re} \varphi(r ; \theta)}{\partial \theta} \frac{\partial \operatorname{Im} \varphi(s ; \theta)}{\partial \theta^{\prime}}+ \\
& +\Omega_{I R, n}(r, s) \frac{\partial \operatorname{Im} \varphi(r ; \theta)}{\partial \theta} \frac{\partial \operatorname{Re} \varphi(s ; \theta)}{\partial \theta^{\prime}}+ \\
& \left.+\Omega_{I I, n}(r, s) \frac{\partial \operatorname{Im} \varphi(r ; \theta)}{\partial \theta} \frac{\partial \operatorname{Im} \varphi(s ; \theta)}{\partial \theta^{\prime}}\right\} d G(r) d G(s),
\end{aligned}
$$


sendo

$$
\begin{aligned}
& \Omega_{R R, n}(r, s)=\frac{1}{n} \sum_{j=1}^{n} \sum_{k=1}^{n} E\left[\left(\cos \left(r^{\prime} Z_{j}\right)-u(r ; \theta)\right)\left(\cos \left(s^{\prime} Z_{k}\right)-u(s ; \theta)\right)\right], \\
& \Omega_{R I, n}(r, s)=\frac{1}{n} \sum_{j=1}^{n} \sum_{k=1}^{n} E\left[\left(\cos \left(r^{\prime} Z_{j}\right)-u(r ; \theta)\right)\left(\sin \left(s^{\prime} Z_{k}\right)-v(s ; \theta)\right)\right], \\
& \Omega_{I R, n}(r, s)=\frac{1}{n} \sum_{j=1}^{n} \sum_{k=1}^{n} E\left[\left(\sin \left(r^{\prime} Z_{j}\right)-v(r ; \theta)\right)\left(\cos \left(s^{\prime} Z_{k}\right)-u(s ; \theta)\right)\right], \\
& \Omega_{I I, n}(r, s)=\frac{1}{n} \sum_{j=1}^{n} \sum_{k=1}^{n} E\left[\left(\sin \left(r^{\prime} Z_{j}\right)-v(r ; \theta)\right)\left(\sin \left(s^{\prime} Z_{k}\right)-v(s ; \theta)\right)\right] .
\end{aligned}
$$

A matriz $\Omega_{n}(r, s)=\left[\begin{array}{ll}\Omega_{R R, n}(r, s) & \Omega_{R I, n}(r, s) \\ \Omega_{I R, n}(r, s) & \Omega_{I I, n}(r, s)\end{array}\right]$ foi obtida no apêndice (B)), e é dada pelas equações (B.33) a (B.36), que reapresentamos abaixo:

$$
\begin{aligned}
\Omega_{R R, n}(r, s)= & \frac{1}{2}\left[\operatorname{Re} \varphi_{z}(r-s)+\operatorname{Re} \varphi_{z}(r+s)\right]-n \operatorname{Re} \varphi_{z}(r) \operatorname{Re} \varphi_{z}(s)+ \\
& +\frac{1}{2 n} \sum_{k=1}^{n-1}(n-k)\left(\operatorname{Re} \Lambda_{k}(r, s)+\operatorname{Re} \Lambda_{k}(r,-s)+\operatorname{Re} \Lambda_{k}(s, r)+\operatorname{Re} \Lambda_{k}(s,-r)\right), \\
\Omega_{R I, n}(r, s)= & \frac{1}{2}\left[\operatorname{Im} \varphi_{z}(-r+s)+\operatorname{Im} \varphi_{z}(r+s)\right]-n \operatorname{Re} \varphi_{z}(r) \operatorname{Im} \varphi_{z}(s)+ \\
& +\frac{1}{2 n} \sum_{k=1}^{n-1}(n-k)\left(\operatorname{Im} \Lambda_{k}(-r, s)+\operatorname{Im} \Lambda_{k}(r, s)+\operatorname{Im} \Lambda_{k}(s,-r)+\operatorname{Im} \Lambda_{k}(s, r)\right), \\
\Omega_{I R, n}(r, s)= & \frac{1}{2}\left[\operatorname{Im} \varphi_{z}(r-s)+\operatorname{Im} \varphi_{z}(r+s)\right]-n \operatorname{Im} \varphi_{z}(r) \operatorname{Re} \varphi_{z}(s)+ \\
& +\frac{1}{2 n} \sum_{k=1}^{n-1}(n-k)\left(\operatorname{Im} \Lambda_{k}(r,-s)+\operatorname{Im} \Lambda_{k}(r, s)+\operatorname{Im} \Lambda_{k}(-s, r)+\operatorname{Im} \Lambda_{k}(s, r)\right), \\
& \frac{1}{2}\left[\operatorname{Re} \varphi_{z}(r-s)-\operatorname{Re} \varphi_{z}(r+s)\right]-n \operatorname{Im} \varphi_{z}(r) \operatorname{Im} \varphi_{z}(s)+ \\
& +\frac{1}{2 n} \sum_{k=1}^{n-1}(n-k)\left(\operatorname{Re} \Lambda_{k}(r,-s)-\operatorname{Re} \Lambda_{k}(r, s)+\operatorname{Re} \Lambda_{k}(s,-r)-\operatorname{Re} \Lambda_{k}(s, r)\right) .
\end{aligned}
$$

Observação: vale notar que as equações (D.13) a (D.16) diferem das equações obtidas por Knight e Yu (2002), pg. 716, pois apresentam um $n$ multiplicando o produto das partes real ou imaginária de $\varphi_{z}(r)$ e $\varphi_{z}(s)$, além de diferentes sinais nos somatórios. Por outro lado, o resultado acima coincide com o apresentado em Knight e Satchell (1997), pg. 176, tomandose o cuidado de notar que a nomenclatura $\Omega^{p}$ utilizada ali refere-se ao nosso $\Omega_{n}(r, s) / n$. 
Podemos, finalmente, escrever $A\left(\theta_{0}\right)$ como

$$
\begin{aligned}
A\left(\theta_{0}\right)= & \lim _{n \rightarrow \infty} 4 \iint\left\{\Omega_{R R, n}(r, s) \frac{\partial \operatorname{Re} \varphi(r ; \theta)}{\partial \theta} \frac{\partial \operatorname{Re} \varphi(s ; \theta)}{\partial \theta^{\prime}}+\right. \\
& +\Omega_{R I, n}(r, s) \frac{\partial \operatorname{Re} \varphi(r ; \theta)}{\partial \theta} \frac{\partial \operatorname{Im} \varphi(s ; \theta)}{\partial \theta^{\prime}}+ \\
& +\Omega_{I R, n}(r, s) \frac{\partial \operatorname{Im} \varphi(r ; \theta)}{\partial \theta} \frac{\partial \operatorname{Re} \varphi(s ; \theta)}{\partial \theta^{\prime}}+ \\
& \left.+\Omega_{I I, n}(r, s) \frac{\partial \operatorname{Im} \varphi(r ; \theta)}{\partial \theta} \frac{\partial \operatorname{Im} \varphi(s ; \theta)}{\partial \theta^{\prime}}\right\} d G(r) d G(s) .
\end{aligned}
$$

Um estimador natural $A_{n}^{(1)}\left(\theta_{0}\right)$ para $A\left(\theta_{0}\right)$ é obtido utilizando-se os valores amostrais em (D.17) e eliminando-se o limite

$$
\begin{aligned}
A_{n}^{(1)}\left(\theta_{0}\right)= & 4 \iint\left\{\Omega_{R R, n}^{(1)}(r, s) \frac{\partial \operatorname{Re} \varphi(r ; \theta)}{\partial \theta} \frac{\partial \operatorname{Re} \varphi(s ; \theta)}{\partial \theta^{\prime}}+\right. \\
& +\Omega_{R I, n}^{(1)}(r, s) \frac{\partial \operatorname{Re} \varphi(r ; \theta)}{\partial \theta} \frac{\partial \operatorname{Im} \varphi(s ; \theta)}{\partial \theta^{\prime}}+ \\
& +\Omega_{I R, n}^{(1)}(r, s) \frac{\partial \operatorname{Im} \varphi(r ; \theta)}{\partial \theta} \frac{\partial \operatorname{Re} \varphi(s ; \theta)}{\partial \theta^{\prime}}+ \\
& \left.+\Omega_{I I, n}^{(1)}(r, s) \frac{\partial \operatorname{Im} \varphi(r ; \theta)}{\partial \theta} \frac{\partial \operatorname{Im} \varphi(s ; \theta)}{\partial \theta^{\prime}}\right\} d G(r) d G(s),
\end{aligned}
$$

em que

$$
\begin{aligned}
& \Omega_{R R, n}^{(1)}(r, s)=\frac{1}{n} \sum_{j=1}^{n} \sum_{k=1}^{n}\left[\cos \left(r^{\prime} Z_{j}\right)-u(r ; \theta)\right]\left[\cos \left(s^{\prime} Z_{k}\right)-u(s ; \theta)\right], \\
& \Omega_{R I, n}^{(1)}(r, s)=\frac{1}{n} \sum_{j=1}^{n} \sum_{k=1}^{n}\left[\cos \left(r^{\prime} Z_{j}\right)-u(r ; \theta)\right]\left[\sin \left(s^{\prime} Z_{k}\right)-v(s ; \theta)\right], \\
& \Omega_{I R, n}^{(1)}(r, s)=\frac{1}{n} \sum_{j=1}^{n} \sum_{k=1}^{n}\left[\sin \left(r^{\prime} Z_{j}\right)-v(r ; \theta)\right]\left[\cos \left(s^{\prime} Z_{k}\right)-u(s ; \theta)\right], \\
& \Omega_{I R, n}^{(1)}(r, s)=\frac{1}{n} \sum_{j=1}^{n} \sum_{k=1}^{n}\left[\sin \left(r^{\prime} Z_{j}\right)-v(r ; \theta)\right]\left[\sin \left(s^{\prime} Z_{k}\right)-v(s ; \theta)\right] .
\end{aligned}
$$

Entretanto, este estimador é muito ineficiente sob o ponto de vista computacional, pois requer $O\left(n^{2}\right)$ operações para cada par $(r, s)$, além de exigir a integração numérica $\mathbb{R}^{p+1}$ em (D.18) .

Podemos obter outro estimador para $A\left(\theta_{0}\right)$ a partir de (D.11) se utilizarmos a média amostral como um estimador para $E\left[K_{1}(\theta) K_{1+k}(\theta)^{\prime}\right]$, de tal forma que

$$
\widehat{E}\left[K_{1}(\theta) K_{1+k}(\theta)^{\prime}\right]=\frac{1}{n-k} \sum_{j=1}^{n-k} K_{j}(\theta) K_{j+k}(\theta)^{\prime}, \quad k=0, \cdots, n-1 .
$$


Substituindo (․19) em (D.11) obtemos o seguinte estimador para $A\left(\theta_{0}\right)$ :

$$
A_{n}^{(2)}\left(\theta_{0}\right)=4\left\{\frac{1}{n} \sum_{j=1}^{n} K_{j}(\theta) K_{j}(\theta)^{\prime}+\sum_{k=1}^{m} \frac{1}{n-k} \sum_{j=1}^{n-k}\left[K_{j}(\theta) K_{j+k}(\theta)^{\prime}+K_{j+k}(\theta) K_{j}(\theta)^{\prime}\right]\right\}
$$

sendo $m$ um número suficientemente grande de modo que o segundo termo da expressão (D.20) tenha aproximadamente convergido, mas bem menor do que $n$, para reduzirmos a ordem das operações. Este algoritmo requer $O(n m)$ operações, e pode ser substancialmente mais rápido, mas ainda requer a obtenção dos vetores $K_{j}$, que são obtidos através de uma integração em $\mathbb{R}^{p+1}$ para cada ponto amostral como em (D.8).

Por outro lado, se pudermos calcular a função $\Lambda_{k}(r, s)$ diretamente, poderemos obter um outro estimador $A_{n}^{(3)}\left(\theta_{0}\right)$ se usarmos em (D.18) os estimadores amostrais dados pelas equações (D.13) a (D.16), sem necessidade da obtenção dos $K_{j}$. Neste caso, o estimador é dado por

$$
\begin{aligned}
A_{n}^{(3)}\left(\theta_{0}\right)= & 4 \iint\left\{\Omega_{R R, n}^{(3)}(r, s) \frac{\partial \operatorname{Re} \varphi(r ; \theta)}{\partial \theta} \frac{\partial \operatorname{Re} \varphi(s ; \theta)}{\partial \theta^{\prime}}+\right. \\
& +\Omega_{R I, n}^{(3)}(r, s) \frac{\partial \operatorname{Re} \varphi(r ; \theta)}{\partial \theta} \frac{\partial \operatorname{Im} \varphi(s ; \theta)}{\partial \theta^{\prime}}+ \\
& +\Omega_{I R, n}^{(3)}(r, s) \frac{\partial \operatorname{Im} \varphi(r ; \theta)}{\partial \theta} \frac{\partial \operatorname{Re} \varphi(s ; \theta)}{\partial \theta^{\prime}}+ \\
& \left.+\Omega_{I I, n}^{(3)}(r, s) \frac{\partial \operatorname{Im} \varphi(r ; \theta)}{\partial \theta} \frac{\partial \operatorname{Im} \varphi(s ; \theta)}{\partial \theta^{\prime}}\right\} d G(r) d G(s),
\end{aligned}
$$

sendo que a matriz $\Omega_{n}^{(3)}(r, s)$ é formada pela seguinte partição:

$$
\Omega_{n}^{(3)}(r, s)=\left[\begin{array}{cc}
\Omega_{R R, n}^{(3)}(r, s) & \Omega_{R I, n}^{(3)}(r, s) \\
\Omega_{I R, n}^{(3)}(r, s) & \Omega_{I I, n}^{(3)}(r, s)
\end{array}\right]
$$


com elementos dados pelas equações (D.13) a (D.16), para $m \leq n$, de tal forma que

$$
\begin{aligned}
\Omega_{R R, m}(r, s)= & \frac{1}{2}\left[\operatorname{Re} \varphi_{z}(r-s)+\operatorname{Re} \varphi_{z}(r+s)\right]-m \operatorname{Re} \varphi_{z}(r) \operatorname{Re} \varphi_{z}(s)+ \\
& +\frac{1}{2 m} \sum_{k=1}^{m-1}(m-k)\left(\operatorname{Re} \Lambda_{k}(r, s)+\operatorname{Re} \Lambda_{k}(r,-s)+\operatorname{Re} \Lambda_{k}(s, r)+\operatorname{Re} \Lambda_{k}(s,-r)\right), \\
\Omega_{R I, m}(r, s)= & \frac{1}{2}\left[\operatorname{Im} \varphi_{z}(-r+s)+\operatorname{Im} \varphi_{z}(r+s)\right]-m \operatorname{Re} \varphi_{z}(r) \operatorname{Im} \varphi_{z}(s)+ \\
& +\frac{1}{2 m} \sum_{k=1}^{m-1}(m-k)\left(\operatorname{Im} \Lambda_{k}(-r, s)+\operatorname{Im} \Lambda_{k}(r, s)+\operatorname{Im} \Lambda_{k}(s,-r)+\operatorname{Im} \Lambda_{k}(s, r)\right), \\
\Omega_{I R, m}(r, s)= & \frac{1}{2}\left[\operatorname{Im} \varphi_{z}(r-s)+\operatorname{Im} \varphi_{z}(r+s)\right]-m \operatorname{Im} \varphi_{z}(r) \operatorname{Re} \varphi_{z}(s)+ \\
& +\frac{1}{2 n} \sum_{k=1}^{m-1}(m-k)\left(\operatorname{Im} \Lambda_{k}(r,-s)+\operatorname{Im} \Lambda_{k}(r, s)+\operatorname{Im} \Lambda_{k}(-s, r)+\operatorname{Im} \Lambda_{k}(s, r)\right), \\
& \frac{1}{2}\left[\operatorname{Re} \varphi_{z}(r-s)-\operatorname{Re} \varphi_{z}(r+s)\right]-n \operatorname{Im} \varphi_{z}(r) \operatorname{Im} \varphi_{z}(s)+ \\
& +\frac{1}{2 m} \sum_{k=1}^{m-1}(m-k)\left(\operatorname{Re} \Lambda_{k}(r,-s)-\operatorname{Re} \Lambda_{k}(r, s)+\operatorname{Re} \Lambda_{k}(s,-r)-\operatorname{Re} \Lambda_{k}(s, r)\right) .
\end{aligned}
$$

Nas equações acima $m$ deve ser escolhido de maneira que a sequiência tenha (aproximadamente) convergido. 



\section{Apêndice E}

\section{Matriz de covariância assintótica do estimador DECF}

Neste capítulo vamos demonstrar as proposições do capítulo 5 referentes ao estimador DECF. Inicialmente relembremos que o estimador DECF $\widehat{\theta}_{n}$ é definido por

$$
\widehat{\theta}_{n}=\arg \min _{\theta \in \Theta} Q_{n}
$$

sendo a função $Q_{n}$ dada por

$$
Q_{n}=\left(V_{n}-V_{\theta}\right)^{\prime} W_{n}\left(V_{n}-V_{\theta}\right),
$$

onde $W_{n}$ é uma matriz $2 q \times 2 q$ simétrica, de posto $\operatorname{rank}\left(W_{n}\right) \leq 2 q$, e

$$
V_{n}-V_{\theta}=\left(\begin{array}{c}
u_{n}\left(r_{1}\right)-u\left(r_{1}\right) \\
\vdots \\
u_{n}\left(r_{q}\right)-u\left(r_{q}\right) \\
v_{n}\left(r_{1}\right)-v\left(r_{1}\right) \\
\vdots \\
v_{n}\left(r_{q}\right)-v\left(r_{q}\right)
\end{array}\right),
$$

e $u_{n}, v_{n}, u$ e $v$ definidos como em (B.2), (B.3), (B.4) e (B.5) respectivamente. Também podemos escrever

$$
V_{n}-V_{\theta}=\frac{1}{n} \sum_{j=1}^{n} f\left(Z_{j} ; \theta\right),
$$


sendo

$$
f\left(Z_{j} ; \theta\right)=\left(\begin{array}{c}
\cos \left(r_{1}^{\prime} Z_{j}\right)-u\left(r_{1} ; \theta\right) \\
\vdots \\
\cos \left(r_{q}^{\prime} Z_{j}\right)-u\left(r_{q} ; \theta\right) \\
\sin \left(r_{1}^{\prime} Z_{j}\right)-v\left(r_{1} ; \theta\right) \\
\vdots \\
\sin \left(r_{q}^{\prime} Z_{j}\right)-v\left(r_{q} ; \theta\right)
\end{array}\right)_{2 q \times 1}
$$

Demonstração do teorema 5.1. Para obter suas propriedades assintóticas, vamos utilizar procedimento análogo ao utilizado no capítulo anterior para o estimador CECF, i.e., vamos utilizar os resultados de Amemiva (1985), obtendo inicialmente $\partial Q_{n} / \partial \theta$ e $\partial^{2} Q_{n} / \partial \theta \partial \theta^{\prime}$.

Aplicando um resultado de Lütkepohl $(2005)^{1}$, obtemos

$$
\frac{\partial Q_{n}}{\partial \theta^{\prime}}=-2\left(V_{n}-V_{\theta}\right)^{\prime} W_{n} \frac{\partial V_{\theta}}{\partial \theta^{\prime}}
$$

$\mathrm{e}$

$$
\frac{\partial^{2} Q_{n}}{\partial \theta \partial \theta^{\prime}}=2\left\{\frac{\partial V_{\theta}^{\prime}}{\partial \theta} W_{n} \frac{\partial V_{\theta}}{\partial \theta^{\prime}}-\left[\left(V_{n}-V_{\theta}\right)^{\prime} W_{n} \otimes I_{m}\right] \frac{\partial}{\partial \theta^{\prime}} \operatorname{vec}\left(\frac{\partial V_{\theta}^{\prime}}{\partial \theta}\right)\right\},
$$

onde $\theta$ é um vetor de dimensão $m \times 1$. Se utilizarmos (E.3) em (E.5) obtemos

$$
\frac{\partial^{2} Q_{n}}{\partial \theta \partial \theta^{\prime}}=2\left\{\frac{\partial V_{\theta}^{\prime}}{\partial \theta} W_{n} \frac{\partial V_{\theta}}{\partial \theta^{\prime}}-\left[\frac{1}{n} \sum_{j=1}^{n} f\left(Z_{j} ; \theta\right)^{\prime} W_{n} \otimes I_{m}\right] \frac{\partial}{\partial \theta^{\prime}} \operatorname{vec}\left(\frac{\partial V_{\theta}^{\prime}}{\partial \theta}\right)\right\} .
$$

Por hipótese os $Z_{j}$ são variáveis aleatórias estacionárias (não independentes) e $\alpha$-mixing; portanto a seqüência $\left\{Z_{j}\right\}$ é ergódica (e.g., proposição 3.44, pg. 48, White (2000)). Podemos então aplicar o teorema ergódico (e.g., teorema 3, pg. 413, Shirvaev (1991), ou teorema 3.34, pg.44, White (2000)):

$$
\frac{1}{n} \sum_{j=1}^{n} f\left(Z_{j} ; \theta\right) \stackrel{a . s .}{\rightarrow} E\left[f\left(Z_{j} ; \theta\right)\right]=0 .
$$

Como por hipótese $W_{n} \stackrel{p}{\rightarrow} W_{0}$ e $\widehat{\theta}_{n} \stackrel{p}{\rightarrow} \theta_{0}$, empregando o resultado acima podemos obter o seguinte resultado para $\frac{\partial^{2} Q_{n}}{\partial \theta \partial \theta^{\prime}}$

$$
\left.\frac{\partial^{2} Q_{n}}{\partial \theta \partial \theta^{\prime}}\right|_{\widehat{\theta}_{n}} \stackrel{p}{\rightarrow} B\left(\theta_{0}\right)=2\left\{\left.\left.\frac{\partial V_{\theta}^{\prime}}{\partial \theta}\right|_{\theta_{0}} W_{0} \frac{\partial V_{\theta}}{\partial \theta^{\prime}}\right|_{\theta_{0}}\right\} .
$$

${ }^{1}$ Lütkepohl (2005), resultado 5, pg. 667: Seja $\Omega$ uma matriz simétrica $n \times n$ e $c(\theta)$ um vetor $n \times 1$ que depende de um vetor $\theta$, de dimensão $m \times 1$. Então

$$
\frac{\partial}{\partial \theta^{\prime}} c(\theta)^{\prime} \Omega c(\theta)=2 c(\theta)^{\prime} \Omega \frac{\partial c(\theta)}{\partial \theta^{\prime}}
$$

e

$$
\frac{\partial^{2}}{\partial \theta \partial \theta^{\prime}} c(\theta)^{\prime} \Omega c(\theta)=2\left\{\frac{\partial c(\theta)^{\prime}}{\partial \theta} \Omega \frac{\partial c(\theta)}{\partial \theta^{\prime}}+\left[c(\theta)^{\prime} \Omega \otimes I_{m}\right] \frac{\partial}{\partial \theta^{\prime}} \operatorname{vec}\left(\frac{\partial c(\theta)^{\prime}}{\partial \theta}\right)\right\},
$$

onde $\otimes$ é o produto de Kronecker, $I_{m}$ é a matriz identidade de dimensão $m \times m$, e $\frac{\partial c(\theta)^{\prime}}{\partial \theta}=\left(\frac{\partial c(\theta)}{\partial \theta^{\prime}}\right)^{\prime}$. 
Com relação a $\partial Q_{n} / \partial \theta$, note que, devido à simetria de $W_{n}$, podemos escrever

$$
\frac{\partial Q_{n}}{\partial \theta}=-2 \frac{\partial V_{\theta}^{\prime}}{\partial \theta} W_{n}\left(V_{n}-V_{\theta}\right)
$$

Como $E\left[V_{n}\right]=V_{\theta}$ temos que

$$
E\left[\frac{\partial Q_{n}}{\partial \theta}\right]=0
$$

$\mathrm{e}$

$$
E\left[\frac{\partial Q_{n}}{\partial \theta \partial \theta^{\prime}}\right]=2 \frac{\partial V_{\theta}^{\prime}}{\partial \theta} W_{n} \frac{\partial V_{\theta}}{\partial \theta^{\prime}} .
$$

Substituindo (E.3) em (E.7) podemos também reescrever $\partial Q_{n} / \partial \theta$ como

$$
\begin{aligned}
\frac{\partial Q_{n}}{\partial \theta} & =-2 \frac{\partial V_{\theta}^{\prime}}{\partial \theta} W_{n}\left(V_{n}-V_{\theta}\right) \\
& =-2 \frac{\partial V_{\theta}^{\prime}}{\partial \theta} W_{n} \frac{1}{n} \sum_{j=1}^{n} f\left(Z_{j} ; \theta\right) \\
& =-\frac{2}{n} \sum_{j=1}^{n} K_{j}(\theta)
\end{aligned}
$$

onde

$$
K_{j}(\theta)=\frac{\partial V_{\theta}^{\prime}}{\partial \theta} W_{n} f\left(Z_{j} ; \theta\right) .
$$

Como no capítulo anterior vamos agora obter as propriedades da seqüência $\sqrt{n} \partial Q_{n} / \partial \theta$ para que possamos aplicar um teorema central limite para seqüências estacionárias. Lembrando que $E\left[\left(\partial Q_{n} / \partial \theta\right)_{\theta_{0}}\right]=0$ temos que

$$
\begin{aligned}
\operatorname{var}\left[\sqrt{n} \frac{\partial Q_{n}}{\partial \theta}\right] & =n E\left[\frac{\partial Q_{n}}{\partial \theta} \frac{\partial Q_{n}}{\partial \theta^{\prime}}\right] \\
& =n E\left[\left(-\frac{2}{n} \sum_{j=1}^{n} K_{j}(\theta)\right)\left(-\frac{2}{n} \sum_{k=1}^{n} K_{k}(\theta)^{\prime}\right)\right] \\
& =\frac{4}{n} E\left[\sum_{j=1}^{n} \sum_{k=1}^{n} K_{j}(\theta) K_{k}(\theta)^{\prime}\right] \\
& =\frac{4}{n} E\left[\sum_{j=1}^{n} K_{j}(\theta) K_{j}(\theta)^{\prime}+\sum_{k=1}^{n-1}(n-k)\left[K_{1}(\theta) K_{1+k}(\theta)^{\prime}+K_{1+k}(\theta) K_{1}(\theta)^{\prime}\right]\right] \\
& =4\left\{E\left[K_{1}(\theta) K_{1}(\theta)^{\prime}\right]+\frac{1}{n} \sum_{k=1}^{n-1}(n-k)\left[K_{1}(\theta) K_{1+k}(\theta)^{\prime}+K_{1+k}(\theta) K_{1}(\theta)^{\prime}\right]\right\} .
\end{aligned}
$$

Os $K_{j}$ são funções contínuas e limitadas dos $Z_{j}$, que são estacionários; portanto a seqüência $\left\{K_{j}(\theta)\right\}$ também é estacionária. Além disso, assumindo condição de $\alpha$-mixing para $Z_{j}$, podemos aplicar o teorema CLT 18.5.4 de Ibragimov e Linnik (1971), obtendo $A\left(\theta_{0}\right)<\infty$, 
sendo

$$
A\left(\theta_{0}\right)=4\left\{E\left[K_{1}\left(\theta_{0}\right) K_{1}\left(\theta_{0}\right)^{\prime}\right]+\sum_{k=1}^{\infty}\left(E\left[K_{1}\left(\theta_{0}\right) K_{1+k}\left(\theta_{0}\right)^{\prime}\right]+E\left[K_{1+k}\left(\theta_{0}\right) K_{1}\left(\theta_{0}\right)^{\prime}\right]\right)\right\}
$$

e se $A\left(\theta_{0}\right) \neq 0$,

$$
\frac{1}{\sqrt{n}}\left[-2 \sum_{j=1}^{n} K_{j}\left(\theta_{0}\right)\right]=\left.\sqrt{n} \frac{\partial Q_{n}}{\partial \theta}\right|_{\theta_{0}} \stackrel{d}{\rightarrow} N\left(0, A\left(\theta_{0}\right)\right) .
$$

Note agora que se usarmos (E.7) poderemos escrever

$$
\begin{aligned}
\operatorname{var}\left[\sqrt{n} \frac{\partial Q_{n}}{\partial \theta}\right] & =n . v a r\left[\frac{\partial Q_{n}}{\partial \theta}\right] \\
& =n . v a r\left[-2 \frac{\partial V_{\theta}^{\prime}}{\partial \theta} W_{n}\left(V_{n}-V_{\theta}\right)\right] \\
& =4 n \frac{\partial V_{\theta}^{\prime}}{\partial \theta} W_{n} \operatorname{var}\left[V_{n}-V_{\theta}\right] W_{n} \frac{\partial V_{\theta}}{\partial \theta^{\prime}} \\
& =4 n \frac{\partial V_{\theta}^{\prime}}{\partial \theta} W_{n}\left(\frac{1}{n} \Omega_{0}\right) W_{n} \frac{\partial V_{\theta}}{\partial \theta^{\prime}} \\
& =4 \frac{\partial V_{\theta}^{\prime}}{\partial \theta} W_{n} \Omega_{0} W_{n} \frac{\partial V_{\theta}}{\partial \theta^{\prime}} .
\end{aligned}
$$

Lembrando que por hipótese $W_{n} \stackrel{p}{\rightarrow} W_{0}$ temos

$$
A\left(\theta_{0}\right)=\left.\left.4 \frac{\partial V_{\theta}^{\prime}}{\partial \theta}\right|_{\theta_{0}} W_{0} \Omega_{0} W_{0} \frac{\partial V_{\theta}}{\partial \theta^{\prime}}\right|_{\theta_{0}} .
$$

O estimador DECF $\widehat{\theta}_{n}$ é obtido através da minimização de (E.1), que pode ser obtida através da solução do sistema

$$
\left.\frac{\partial Q_{n}}{\partial \theta}\right|_{\widehat{\theta}_{n}}=0
$$

Expandindo $\partial Q_{n} / \partial \theta$ em uma série de Taylor em torno do vetor de parâmetros, $\theta_{0}$, obtemos

$$
\left.\frac{\partial Q_{n}}{\partial \theta}\right|_{\widehat{\theta}_{n}}=\left.\frac{\partial Q_{n}}{\partial \theta}\right|_{\theta_{0}}+\left.\frac{\partial^{2} Q_{n}}{\partial \theta \partial \theta^{\prime}}\right|_{\theta^{*}}\left(\widehat{\theta}_{n}-\theta_{0}\right)
$$

onde $\theta^{*}$ fica entre $\widehat{\theta}_{n}$ e $\theta_{0}$. Como $\left.\frac{\partial Q_{n}}{\partial \theta}\right|_{\widehat{\theta}_{n}}=0$ pela definição de $\widehat{\theta}_{n}$, se multiplicarmos ambos os lados por $\sqrt{n}\left[\left.\frac{\partial^{2} Q_{n}}{\partial \theta \partial \theta^{\prime}}\right|_{\theta^{*}}\right]^{-1}$ obtemos

$$
\sqrt{n}\left(\widehat{\theta}_{n}-\theta_{0}\right)=-\left[\left.\frac{\partial^{2} Q_{n}}{\partial \theta \partial \theta^{\prime}}\right|_{\theta^{*}}\right]^{-1}\left(\left.\sqrt{n} \frac{\partial Q_{n}}{\partial \theta}\right|_{\theta_{0}}\right)
$$


Agora, como por hipótese $\widehat{\theta}_{n} \stackrel{p}{\rightarrow} \theta_{0}$, também $\theta^{*} \stackrel{p}{\rightarrow} \theta_{0}$, e consequentemente

$$
\left.\frac{\partial^{2} Q_{n}}{\partial \theta \partial \theta^{\prime}}\right|_{\theta^{*}} \stackrel{p}{\rightarrow} B\left(\theta_{0}\right)
$$

Usando os resultados (E.8) e (E.10), obtemos a distribuição limite do estimador DECF $\widehat{\theta}_{n}$ através de

$$
\sqrt{n}\left(\widehat{\theta}_{n}-\theta_{0}\right) \stackrel{d}{\longrightarrow} N\left(0, B\left(\theta_{0}\right)^{-1} A\left(\theta_{0}\right) B\left(\theta_{0}\right)^{-1}\right)
$$

sendo

$$
B\left(\theta_{0}\right)^{-1} A\left(\theta_{0}\right) B\left(\theta_{0}\right)^{-1}=\left(\frac{\partial V_{\theta}^{\prime}}{\partial \theta} W_{0} \frac{\partial V_{\theta}}{\partial \theta^{\prime}}\right)^{-1} \frac{\partial V_{\theta}^{\prime}}{\partial \theta} W_{0} \Omega_{0} W_{0} \frac{\partial V_{\theta}}{\partial \theta^{\prime}}\left(\frac{\partial V_{\theta}^{\prime}}{\partial \theta} W_{0} \frac{\partial V_{\theta}}{\partial \theta^{\prime}}\right)^{-1}
$$





\section{Apêndice F}

\section{Filtro de Gram-Charlier}

Seja o seguinte modelo de espaço de estados univariado:

$$
\begin{gathered}
X_{t}=\delta+\phi X_{t-1}+\eta_{t}, \\
Y_{t}=\alpha+\beta X_{t}+\varepsilon_{t}, \\
\eta_{t} \sim \operatorname{NIID}\left(0, \sigma_{\eta}^{2}\right), \\
\varepsilon_{t} \sim \text { alguma distribuição i.i.d. com momentos finitos até } 4^{a} \text { ordem. }
\end{gathered}
$$

Adotaremos a seguinte notação, análoga à empregada em Shumway e Stoffer (2000):

$$
\begin{aligned}
\mu_{t}^{s} & =E\left[X_{t} \mid y_{1: s}\right], \\
v_{t}^{s} & =\left(\sigma_{t}^{s}\right)^{2}=E\left[\left(X_{t}-\mu_{t}^{s}\right)^{2} \mid y_{1: s}\right], \\
\gamma_{1, t}^{s} & =\frac{E\left[\left(X_{t}-\mu_{t}^{s}\right)^{3} \mid y_{1: s}\right]}{\left(\sigma_{t}^{s}\right)^{3}}, \\
\gamma_{2, t}^{s} & =\frac{E\left[\left(X_{t}-\mu_{t}^{s}\right)^{4} \mid y_{1: s}\right]}{\left(\sigma_{t}^{s}\right)^{4}}-3 .
\end{aligned}
$$

Vamos assumir que a distribuição condicional de $x_{t-1} \mid y_{1: t-1}$ possa ser aproximada por uma expansão de Gram-Charlier até a $4^{a}$ ordem $^{1}$, i.e.,

$$
p\left(x_{t-1} \mid y_{1: t-1}\right) \cong \frac{e^{-\frac{w^{2}}{2}}}{\sqrt{2 \pi} \sigma_{t-1}^{t-1}}\left\{1+\frac{\gamma_{1, t-1}^{t-1}}{6}\left(w^{3}-3 w\right)+\frac{\gamma_{2, t-1}^{t-1}}{24}\left(w^{4}-6 w^{2}+3\right)\right\}
$$

\footnotetext{
${ }^{1} \mathrm{~A}$ expansão de Edgeworth até $4^{a}$ ordem possui um termo adicional à expansão de Gram-Charlier, de maneira que a distribuição condicional de $x_{t-1} \mid y_{1: t-1}$ seria aproximada por:

$p\left(x_{t-1} \mid y_{1: t-1}\right) \cong \frac{e^{-\frac{w^{2}}{2}}}{\sqrt{2 \pi} \sigma_{t-1}^{t-1}}\left\{1+\frac{\gamma_{1, t-1}^{t-1}}{6}\left(w^{3}-3 w\right)+\frac{\gamma_{2, t-1}^{t-1}}{24}\left(w^{4}-6 w^{2}+3\right)+\frac{\left(\gamma_{2, t-1}^{t-1}\right)^{2}}{72}\left(w^{6}-15 w^{4}+45 w^{2}-15\right)\right\}$
}

Neste trabalho, entretanto, vamos utilizar a expansão de Gram-Charlier. 
onde

$$
w=\frac{x_{t-1}-\mu_{t-1}^{t-1}}{\sigma_{t-1}^{t-1}} .
$$

Por outro lado, a distribuição de $x_{t} \mid x_{t-1}$ é dada por hipótese por:

$$
p\left(x_{t} \mid x_{t-1}\right)=\frac{e^{-\frac{1}{2}\left(\frac{x_{t}-\delta-\phi x_{t-1}}{\sigma_{\eta}}\right)^{2}}}{\sqrt{2 \pi} \sigma_{\eta}} .
$$

Para calcular a distribuição de $x_{t} \mid y_{1: t-1}$, isto é, a previsão para o passo seguinte $(t)$ condicional na informação disponível até $t-1$, precisamos efetuar a seguinte integração:

$$
p\left(x_{t} \mid y_{1: t-1}\right)=\int p\left(x_{t} \mid x_{t-1}\right) p\left(x_{t-1} \mid y_{1: t-1}\right) d x_{t-1} .
$$

Inicialmente vamos calcular

$$
\begin{aligned}
& p\left(x_{t} \mid x_{t-1}\right) p\left(x_{t-1} \mid y_{1: t-1}\right) \cong \frac{e^{-\frac{1}{2}\left(\frac{x_{t}-\delta-\phi x_{t-1}}{\sigma \eta}\right)^{2}}}{\sqrt{2 \pi} \sigma_{\eta}} \frac{e^{-\frac{w^{2}}{2}}}{\sqrt{2 \pi} \sigma_{t-1}^{t-1}}\left\{1+\frac{\gamma_{1, t-1}^{t-1}}{6}\left(w^{3}-3 w\right)+\right. \\
& \left.+\frac{\gamma_{2, t-1}^{t-1}}{24}\left(w^{4}-6 w^{2}+3\right)\right\} \\
& =\frac{1}{2 \pi \sigma_{\eta} \sigma_{t-1}^{t-1}} \exp \left\{-\frac{1}{2}\left(\frac{x_{t}-\delta-\phi x_{t-1}}{\sigma_{\eta}}\right)^{2}-\frac{1}{2} w^{2}\right\}\{\cdots\} \\
& =\frac{1}{2 \pi \sigma_{\eta} \sigma_{t-1}^{t-1}} \exp \left\{-\frac{1}{2}\left(\frac{x_{t}-\delta-\phi\left(\mu_{t-1}^{t-1}+\sigma_{t-1}^{t-1} w\right)}{\sigma_{\eta}}\right)^{2}-\frac{1}{2} w^{2}\right\}\{\cdots\} \\
& =\frac{1}{2 \pi \sigma_{\eta} \sigma_{t-1}^{t-1}} \exp \left\{-\frac{1}{2}\left(\frac{x_{t}-\delta-\phi \mu_{t-1}^{t-1}-\phi \sigma_{t-1}^{t-1} w}{\sigma_{\eta}}\right)^{2}-\frac{1}{2} w^{2}\right\}\{\cdots\} \\
& =\frac{1}{2 \pi \sigma_{\eta} \sigma_{t-1}^{t-1}} \exp \left\{-\frac{1}{2}\left[\frac{\left(x_{t}-\delta-\phi \mu_{t-1}^{t-1}\right)^{2}-2\left(x_{t}-\delta-\phi \mu_{t-1}^{t-1}\right) \phi \sigma_{t-1}^{t-1} w+\phi^{2} v_{t-1}^{t-1} w^{2}}{\sigma_{\eta}^{2}}+w^{2}\right]\right\}\{\cdots\} \\
& =\frac{1}{2 \pi \sigma_{\eta} \sigma_{t-1}^{t-1}} \exp \left\{-\frac{1}{2}\left[\frac{\left(x_{t}-\delta-\phi \mu_{t-1}^{t-1}\right)^{2}}{\sigma_{\eta}^{2}}-\frac{2 \phi \sigma_{t-1}^{t-1}\left(x_{t}-\delta-\phi \mu_{t-1}^{t-1}\right)}{\sigma_{\eta}^{2}} w+\frac{\phi^{2} v_{t-1}^{t-1}}{\sigma_{\eta}^{2}} w^{2}+w^{2}\right]\right\}\{\cdots\} \\
& =\frac{1}{2 \pi \sigma_{\eta} \sigma_{t-1}^{t-1}} \exp \left\{-\frac{1}{2}\left[\left(\frac{\phi^{2} v_{t-1}^{t-1}}{\sigma_{\eta}^{2}}+1\right) w^{2}-2\left(\frac{\phi \sigma_{t-1}^{t-1}\left(x_{t}-\delta-\phi \mu_{t-1}^{t-1}\right)}{\sigma_{\eta}^{2}}\right) w+\right]\right\}\{\cdots\} \\
& \left.\left.+\frac{\left(x_{t}-\delta-\phi \mu_{t-1}^{t-1}\right)^{2}}{\sigma_{\eta}^{2}}\right]\right\}\{\cdots\} \text {. }
\end{aligned}
$$


Observe agora que podemos escrever

$$
\begin{aligned}
y & =a w^{2}-2 b w+c \\
& =a\left[w^{2}-2 \frac{b}{a} w+\frac{c}{a}\right] \\
& =a\left[\left(w-\frac{b}{a}\right)^{2}-\left(\frac{b}{a}\right)^{2}+\frac{c}{a}\right] \\
& =a\left(w-\frac{b}{a}\right)^{2}+c-\frac{b^{2}}{a},
\end{aligned}
$$

sendo que para (F.13) temos

$$
\begin{gathered}
a=\frac{\phi^{2} v_{t-1}^{t-1}}{\sigma_{\eta}^{2}}+1 \\
=\frac{\phi^{2} v_{t-1}^{t-1}+\sigma_{\eta}^{2}}{\sigma_{\eta}^{2}}, \\
b=\frac{\phi \sigma_{t-1}^{t-1}\left(x_{t}-\delta-\phi \mu_{t-1}^{t-1}\right)}{\sigma_{\eta}^{2}} \\
c=\frac{\left(x_{t}-\delta-\phi \mu_{t-1}^{t-1}\right)^{2}}{\sigma_{\eta}^{2}}, \\
\frac{b^{2}}{a=} \frac{\left(x_{t}-\delta-\phi \mu_{t-1}^{t-1}\right)^{2}}{\sigma_{\eta}^{2}}-\frac{\phi^{2} v_{t-1}^{t-1}\left(x_{t}-\delta-\phi \mu_{t-1}\right)^{2}}{\left(\phi^{2} v_{t-1}^{t-1}+\sigma_{\eta}^{2}\right) \sigma_{\eta}^{2}} \\
=\left(\frac{\phi^{2} v_{t-1}^{t-1}+\sigma_{\eta}^{2}-\phi^{2} v_{t-1}^{t-1}}{\phi^{2} v_{t-1}^{t-1}+\sigma_{\eta}^{2}}\right) \frac{\left(x_{t}-\delta-\phi \mu_{t-1}^{t-1}\right)^{2}}{\sigma_{\eta}^{2}} \\
=\frac{\left(x_{t}-\delta-\phi \mu_{t-1}^{t-1}\right)^{2}}{\phi^{2} v_{t-1}^{t-1}+\sigma_{\eta}^{2}} .
\end{gathered}
$$

Usando (F.14) a (․16) podemos escrever (․12) como

$$
\begin{aligned}
p\left(x_{t} \mid y_{1: t-1}\right) & =\int p\left(x_{t} \mid x_{t-1}\right) p\left(x_{t-1} \mid y_{1: t-1}\right) d x_{t-1} \\
& \cong \int \frac{e^{-\frac{1}{2}\left[a\left(w-\frac{b}{a}\right)^{2}+c-\frac{b^{2}}{a}\right]}}{2 \pi \sigma_{\eta} \sigma_{t-1}^{t-1}}\left\{1+\frac{\gamma_{1, t-1}^{t-1}}{6}\left(w^{3}-3 w\right)+\frac{\gamma_{2, t-1}^{t-1}}{24}\left(w^{4}-6 w^{2}+3\right)\right\} d x_{t-1} .
\end{aligned}
$$

Utilizando

$$
d w=\frac{d x_{t-1}}{\sigma_{t-1}^{t-1}}
$$


podemos escrever (F.18) como

$$
\begin{aligned}
p\left(x_{t} \mid y_{1: t-1}\right) & \cong \int \frac{e^{-\frac{1}{2}\left[a\left(w-\frac{b}{a}\right)^{2}+c-\frac{b^{2}}{a}\right]}}{2 \pi \sigma_{\eta}}\left\{1+\frac{\gamma_{1, t-1}^{t-1}}{6}\left(w^{3}-3 w\right)+\frac{\gamma_{2, t-1}^{t-1}}{24}\left(w^{4}-6 w^{2}+3\right)\right\} d w \\
& =\frac{e^{-\frac{1}{2}\left[c-\frac{b^{2}}{a}\right]}}{\sqrt{2 \pi} \sigma_{\eta} \sqrt{a}} \int \frac{e^{-\frac{1}{2}\left[\left(\frac{w-\frac{b}{a}}{1 / \sqrt{a}}\right)^{2}\right]}}{\sqrt{2 \pi}(1 / \sqrt{a})}\left\{1+\frac{\gamma_{1, t-1}^{t-1}}{6}\left(w^{3}-3 w\right)+\frac{\gamma_{2, t-1}^{t-1}}{24}\left(w^{4}-6 w^{2}+3\right)\right\} d w .
\end{aligned}
$$

Note que a expressão (F.20) envolve os momentos não centrais da distribuição de $w$, que é uma normal de média $\frac{b}{a}$ e variância $\frac{1}{a}$, i.e., $w \sim N\left(\frac{b}{a}, \frac{1}{a}\right)$. Para obter estes momentos vamos utilizar uma conhecida relação recursiva entre momentos não centrais e cumulantes (Smith (1995)) dada por:

$$
\mu_{n}^{\prime}=\kappa_{n}+\sum_{i=1}^{n-1}\left(\begin{array}{c}
n-1 \\
i-1
\end{array}\right) \kappa_{i} \mu_{n-i}^{\prime}
$$

Para o caso de uma variável aleatórial normal, os cumulantes maiores do que 2 são iguais a zero (Stuart e Ord (1994, pg.93)). Assim, se $Z \sim N\left(\mu, \sigma^{2}\right)$ temos que $\kappa_{1}=\mu, \kappa_{2}=\sigma^{2}$, e $\kappa_{i}=0, i \geq 3$. Utilizando este resultado em (F.21) obtemos:

$$
\mu_{n}^{\prime}=\mu \mu_{n-1}^{\prime}+(n-1) \sigma^{2}, \quad n \geq 3 .
$$

Os momentos não centrais da normal podem ser facilmente obtidos utilizando-se (F.22). Por exemplo,

$$
\begin{aligned}
\mu_{2}^{\prime}= & \mu^{2}+\sigma^{2} \\
\mu_{3}^{\prime}= & \mu^{3}+3 \mu \sigma^{2} \\
\mu_{4}^{\prime}= & \mu^{4}+6 \mu^{2} \sigma^{2}+3 \sigma^{4} \\
\mu_{5}^{\prime}= & \mu^{5}+10 \mu^{3} \sigma^{2}+15 \mu \sigma^{4} \\
\mu_{6}^{\prime}= & \mu^{6}+15 \mu^{4} \sigma^{2}+45 \mu^{2} \sigma^{4}+15 \sigma^{6} \\
& \text { etc. }
\end{aligned}
$$


Aplicando os resultados acima em (F.20) obtemos,

$$
\begin{aligned}
p\left(x_{t} \mid y_{1: t-1}\right) \cong & \frac{e^{-\frac{1}{2}\left[c-\frac{b^{2}}{a}\right]}}{\sqrt{2 \pi} \sigma_{\eta} \sqrt{a}} \int \frac{e^{-\frac{1}{2}\left[\left(\frac{w-\frac{b}{a}}{1 / \sqrt{a}}\right)^{2}\right]}}{\sqrt{2 \pi}(1 / \sqrt{a})}\left\{1+\frac{\gamma_{1, t-1}^{t-1}}{6}\left(w^{3}-3 w\right)+\frac{\gamma_{2, t-1}^{t-1}}{24}\left(w^{4}-6 w^{2}+3\right)\right\} d w \\
= & \frac{e^{-\frac{1}{2}\left[c-\frac{b^{2}}{a}\right]}}{\sqrt{2 \pi} \sigma_{\eta} \sqrt{a}}\left\{1+\frac{\gamma_{1, t-1}^{t-1}}{6}\left[\left(3 \frac{b}{a}\left(\frac{1}{\sqrt{a}}\right)^{2}+\left(\frac{b}{a}\right)^{3}\right)-3\left(\frac{b}{a}\right)\right]+\right. \\
& \left.\left.+\frac{\gamma_{2, t-1}^{t-1}\left[\left(3\left(\frac{1}{\sqrt{a}}\right)^{4}+6\left(\frac{b}{a}\right)^{2}\left(\frac{1}{\sqrt{a}}\right)^{2}+\left(\frac{b}{a}\right)^{4}\right)-\right.}{}-6\left(\left(\frac{1}{\sqrt{a}}\right)^{2}+\left(\frac{b}{a}\right)^{2}\right)+3\right]\right\} \\
= & \frac{\left.e^{-\frac{1}{2}\left[c-\frac{b^{2}}{a}\right.}\right]}{\sqrt{2 \pi} \sigma_{\eta} \sqrt{a}}\left\{1+\frac{\gamma_{1, t-1}^{t-1}}{6}\left[3 \frac{b}{a^{2}}+\left(\frac{b}{a}\right)^{3}-3 \frac{b}{a}\right]+\right. \\
& \left.+\frac{\gamma_{2, t-1}^{t-1}}{24}\left[\frac{3}{a^{2}}+6\left(\frac{b}{a}\right)^{2} \frac{1}{a}+\left(\frac{b}{a}\right)^{4}-\frac{6}{a}-6\left(\frac{b}{a}\right)^{2}+3\right]\right\}
\end{aligned}
$$

e finalmente,

$$
\begin{aligned}
p\left(x_{t} \mid y_{1: t-1}\right) \cong & \frac{e^{-\frac{1}{2}\left[c-\frac{b^{2}}{a}\right]}}{\sqrt{2 \pi} \sigma_{\eta} \sqrt{a}}\left\{1+\frac{\gamma_{1, t-1}^{t-1}}{6} \frac{\left(3 a b+b^{3}-3 a^{2} b\right)}{a^{3}}+\right. \\
& \left.+\frac{\gamma_{2, t-1}^{t-1}}{24} \frac{\left[b^{4}+3\left(a^{2}+2 a b^{2}-2 a^{2} b^{2}-2 a^{3}+a^{4}\right)\right]}{a^{4}}\right\} .
\end{aligned}
$$

Note agora que podemos escrever

$$
\begin{gathered}
\frac{3 a b+b^{3}-3 a^{2} b}{a^{3}}=\left(\frac{b}{a}\right)^{3}-3\left(\frac{a-1}{a}\right)\left(\frac{b}{a}\right) \\
\frac{b^{4}+3\left(a^{2}+2 a b^{2}-2 a^{2} b^{2}-2 a^{3}+a^{4}\right)}{a^{4}}=\left(\frac{b}{a}\right)^{4}-6\left(\frac{a-1}{a}\right)\left(\frac{b}{a}\right)^{2}+3\left(\frac{a-1}{a}\right)^{2} .
\end{gathered}
$$

Usando agora (F.14) e (F.15) obtemos

$$
\begin{gathered}
\frac{b}{a}=\frac{\phi \sigma_{t-1}^{t-1}\left(x_{t}-\delta-\phi \mu_{t-1}^{t-1}\right)}{\phi^{2} v_{t-1}^{t-1}+\sigma_{\eta}^{2}}, \\
\frac{a-1}{a}=\frac{\phi^{2} v_{t-1}^{t-1}}{\phi^{2} v_{t-1}^{t-1}+\sigma_{\eta}^{2}}
\end{gathered}
$$

Chamemos

$$
\zeta=\frac{x_{t}-\delta-\phi \mu_{t-1}^{t-1}}{\sqrt{\phi^{2} v_{t-1}^{t-1}+\sigma_{\eta}^{2}}}
$$


Usando as equações acima obtemos

$$
\begin{gathered}
\left(\frac{b}{a}\right)^{3}-3\left(\frac{a-1}{a}\right)\left(\frac{b}{a}\right)= \\
=\frac{\left(\phi \sigma_{t-1}^{t-1}\right)^{3} \zeta^{3}}{\left(\phi^{2} v_{t-1}^{t-1}+\sigma_{\eta}^{2}\right)^{3 / 2}}-3\left(\frac{\phi^{2} v_{t-1}^{t-1}}{\phi^{2} v_{t-1}^{t-1}+\sigma_{\eta}^{2}}\right) \frac{\phi \sigma_{t-1}^{t-1} \zeta}{\left(\phi^{2} v_{t-1}^{t-1}+\sigma_{\eta}^{2}\right)^{1 / 2}} \\
=\frac{\left(\phi \sigma_{t-1}^{t-1}\right)^{3}}{\left(\phi^{2} v_{t-1}^{t-1}+\sigma_{\eta}^{2}\right)^{3 / 2}}\left(\zeta^{3}-3 \zeta\right)
\end{gathered}
$$

e

$$
\begin{gathered}
\left(\frac{b}{a}\right)^{4}-6\left(\frac{a-1}{a}\right)\left(\frac{b}{a}\right)^{2}+3\left(\frac{a-1}{a}\right)^{2}= \\
=\frac{\left(\phi \sigma_{t-1}^{t-1}\right)^{4} \zeta^{4}}{\left(\phi^{2} v_{t-1}^{t-1}+\sigma_{\eta}^{2}\right)^{2}}-6\left(\frac{\phi^{2} v_{t-1}^{t-1}}{\phi^{2} v_{t-1}^{t-1}+\sigma_{\eta}^{2}}\right) \frac{\left(\phi \sigma_{t-1}^{t-1}\right)^{2} \zeta^{2}}{\phi^{2} v_{t-1}^{t-1}+\sigma_{\eta}^{2}}+3 \frac{\left(\phi^{2} v_{t-1}^{t-1}\right)^{2}}{\left(\phi^{2} v_{t-1}^{t-1}+\sigma_{\eta}^{2}\right)^{2}} \\
=\frac{\left(\phi \sigma_{t-1}^{t-1}\right)^{4}}{\left(\phi^{2} v_{t-1}^{t-1}+\sigma_{\eta}^{2}\right)^{2}}\left(\zeta^{4}-6 \zeta^{2}+3\right) .
\end{gathered}
$$

Note também por (F.17) que podemos escrever

$$
c-\frac{b^{2}}{a}=\frac{\left(x_{t}-\delta-\phi \mu_{t-1}^{t-1}\right)^{2}}{\phi^{2} v_{t-1}^{t-1}+\sigma_{\eta}^{2}}=\zeta^{2} .
$$

Substituindo os resultados acima em ( $(\underline{F .23})$ obtemos

$$
\begin{aligned}
p\left(x_{t} \mid y_{1: t-1}\right) \cong & \frac{e^{-\frac{1}{2} \zeta^{2}}}{\sqrt{2 \pi} \sigma_{\eta} \sqrt{\frac{\phi^{2} v_{t-1}^{t-1}+\sigma_{\eta}^{2}}{\sigma_{\eta}^{2}}}}\left\{1+\frac{\gamma_{1, t-1}^{t-1}}{6} \frac{\left(\phi \sigma_{t-1}^{t-1}\right)^{3}}{\left(\phi^{2} v_{t-1}^{t-1}+\sigma_{\eta}^{2}\right)^{3 / 2}}\left(\zeta^{3}-3 \zeta\right)+\right. \\
& \left.+\frac{\gamma_{2, t-1}^{t-1}}{24} \frac{\left(\phi \sigma_{t-1}^{t-1}\right)^{4}}{\left(\phi^{2} v_{t-1}^{t-1}+\sigma_{\eta}^{2}\right)^{2}}\left(\zeta^{4}-6 \zeta^{2}+3\right)\right\} .
\end{aligned}
$$

Assim obtemos a distribuição de $x_{t} \mid y_{1: t-1}$ por

$$
p\left(x_{t} \mid y_{1: t-1}\right) \cong \frac{e^{-\frac{1}{2} \zeta^{2}}}{\sqrt{2 \pi} \sigma_{t}^{t-1}}\left\{1+\frac{\gamma_{1, t}^{t-1}}{6}\left(\zeta^{3}-3 \zeta\right)+\frac{\gamma_{2, t}^{t-1}}{24}\left(\zeta^{4}-6 \zeta^{2}+3\right)\right\}
$$


onde

$$
\begin{aligned}
\mu_{t}^{t-1} & =\delta+\phi \mu_{t-1}^{t-1}, \\
v_{t}^{t-1} & =\left(\sigma_{t}^{t-1}\right)^{2}=\phi^{2} v_{t-1}^{t-1}+\sigma_{\eta}^{2}, \\
\gamma_{1, t}^{t-1} & =\gamma_{1, t-1}^{t-1}\left(\frac{\phi \sigma_{t-1}^{t-1}}{\sqrt{\phi^{2} v_{t-1}^{t-1}+\sigma_{\eta}^{2}}}\right)^{3}=\gamma_{1, t-1}^{t-1}\left(\frac{\phi \sigma_{t-1}^{t-1}}{\sigma_{t}^{t-1}}\right)^{3}, \\
\gamma_{2, t}^{t-1} & =\gamma_{2, t-1}^{t-1}\left(\frac{\phi \sigma_{t-1}^{t-1}}{\sqrt{\phi^{2} v_{t-1}^{t-1}+\sigma_{\eta}^{2}}}\right)^{4}=\gamma_{2, t-1}^{t-1}\left(\frac{\phi \sigma_{t-1}^{t-1}}{\sigma_{t}^{t-1}}\right)^{4}, \\
\zeta & =\frac{x_{t}-\delta-\phi \mu_{t-1}^{t-1}}{\sqrt{\phi^{2} v_{t-1}^{t-1}+\sigma_{\eta}^{2}}}=\frac{x_{t}-\mu_{t}^{t-1}}{\sigma_{t}^{t-1}} .
\end{aligned}
$$

Podemos notar pela equação (F.27) que a distribuição de $x_{t} \mid y_{1: t-1}$, isto é, a previsão para a variável latente no passo seguinte $(t)$, condicional à informação disponível até $t-1$, também pode ser aproximada por uma expansão de Gram-Charlier, com momentos centrais dados pelas equações (F.28) a (

A distribuição conjunta de $x_{t}, y_{t} \mid y_{1: t-1}$ pode ser obtida por

$$
p\left(x_{t}, y_{t} \mid y_{1: t-1}\right)=p\left(y_{t} \mid x_{t}\right) p\left(x_{t} \mid y_{1: t-1}\right) .
$$

Por um lado, podemos aproximar a densidade condicional de $x_{t} \mid y_{1: t-1}$ por (F.27). Por outro lado, podemos obter verossimilhança amostral $p\left(y_{t} \mid x_{t}\right)$ a partir da distribuição dos erros $\varepsilon_{t}$, $p\left(\varepsilon_{t}\right)$, através do método do jacobiano:

$$
p_{y}\left(y_{t}\right)=p_{\varepsilon}\left(g^{-1}\left(y_{t}\right)\right)\left|\frac{d}{d y_{t}} g^{-1}\left(y_{t}\right)\right|,
$$

onde

$$
g\left(\varepsilon_{t}\right)=y_{t}=\alpha+\beta x_{t}+\varepsilon_{t}
$$

Como

$$
\frac{d}{d y_{t}} g^{-1}\left(y_{t}\right)=\frac{d}{d y_{t}}\left(y_{t}-\alpha-\beta x_{t}\right)=1
$$

temos que

$$
p_{y}\left(y_{t}\right)=p_{\varepsilon}\left(y_{t}-\alpha-\beta x_{t}\right) .
$$

Vamos assumir agora que a densidade dos erros $p\left(\varepsilon_{t}\right)$ também possa ser aproximada por 
uma expansão de Gram-Charlier até a $4^{a}$ ordem, i.e.,

$$
\begin{aligned}
p\left(\varepsilon_{t}\right) \cong & \frac{e^{-\frac{1}{2}\left(\frac{\varepsilon_{t}-\mu_{\varepsilon}}{\sigma_{\varepsilon}}\right)^{2}}}{\sqrt{2 \pi} \sigma_{\varepsilon}}\left\{1+\left[\frac{\gamma_{1, \varepsilon}}{6}\left(\frac{\varepsilon_{t}-\mu_{\varepsilon}}{\sigma_{\varepsilon}}\right)^{3}-3\left(\frac{\varepsilon_{t}-\mu_{\varepsilon}}{\sigma_{\varepsilon}}\right)\right]+\right. \\
& \left.+\frac{\gamma_{2, \varepsilon}}{24}\left[\left(\frac{\varepsilon_{t}-\mu_{\varepsilon}}{\sigma_{\varepsilon}}\right)^{4}-6\left(\frac{\varepsilon_{t}-\mu_{\varepsilon}}{\sigma_{\varepsilon}}\right)^{2}+3\right]\right\} .
\end{aligned}
$$

Utilizando (F.36) e (F.37) podemos escrever

$$
\begin{aligned}
p\left(y_{t} \mid x_{t}\right) \cong & \frac{e^{-\frac{1}{2}\left(\frac{y_{t}-\mu_{\varepsilon}-\alpha-\beta x_{t}}{\sigma_{\varepsilon}}\right)^{2}}}{\sqrt{2 \pi} \sigma_{\varepsilon}}\left\{1+\left[\frac{\gamma_{1, \varepsilon}}{6}\left(\frac{y_{t}-\mu_{\varepsilon}-\alpha-\beta x_{t}}{\sigma_{\varepsilon}}\right)^{3}-3\left(\frac{y_{t}-\mu_{\varepsilon}-\alpha-\beta x_{t}}{\sigma_{\varepsilon}}\right)\right]\right. \\
& \left.+\frac{\gamma_{2, \varepsilon}}{24}\left[\left(\frac{y_{t}-\mu_{\varepsilon}-\alpha-\beta x_{t}}{\sigma_{\varepsilon}}\right)^{4}-6\left(\frac{y_{t}-\mu_{\varepsilon}-\alpha-\beta x_{t}}{\sigma_{\varepsilon}}\right)^{2}+3\right]\right\} .
\end{aligned}
$$

No que segue é conveniente escrevermos (F.38) em função de $\zeta$, conforme (F.32). Assim vamos inicialmente expandir as potências dentro dos colchetes em (F.38):

$$
\begin{aligned}
y_{t}-\mu_{\varepsilon}-\alpha-\beta x_{t} & =y_{t}-\mu_{\varepsilon}-\alpha-\beta\left(\mu_{\zeta}+\sigma_{\zeta} \zeta\right) \\
& =\rho_{t}-\beta \sigma_{\zeta} \zeta,
\end{aligned}
$$

onde, para facilitar a notação, definimos

$$
\begin{aligned}
\rho_{t} & =y_{t}-\mu_{\varepsilon}-\alpha-\beta \mu_{\zeta}, \\
\mu_{\zeta} & =\mu_{t}^{t-1}, \\
\sigma_{\zeta} & =\sigma_{t}^{t-1} .
\end{aligned}
$$

Analogamente para as outras potências temos

$$
\begin{aligned}
\left(y_{t}-\mu_{\varepsilon}-\alpha-\beta x_{t}\right)^{2} & =\left(\rho_{t}-\beta \sigma_{\zeta} \zeta\right)^{2} \\
= & \rho_{t}^{2}-2 \rho_{t} \beta \sigma_{\zeta} \zeta+\beta^{2} \sigma_{\zeta}^{2} \zeta^{2}, \\
\left(y_{t}-\mu_{\varepsilon}-\alpha-\beta x_{t}\right)^{3}= & \left(\rho_{t}-\beta \sigma_{\zeta} \zeta\right)^{3} \\
= & \rho_{t}^{3}-3 \rho_{t}^{2} \beta \sigma_{\zeta} \zeta+3 \rho_{t} \beta^{2} \sigma_{\zeta}^{2} \zeta^{2}-\beta^{3} \sigma_{\zeta}^{3} \zeta^{3}, \\
\left(y_{t}-\mu_{\varepsilon}-\alpha-\beta x_{t}\right)^{4}= & \left(\rho_{t}-\beta \sigma_{\zeta} \zeta\right)^{4} \\
= & \rho_{t}^{4}-4 \rho_{t}^{3} \beta \sigma_{\zeta} \zeta+6 \rho_{t}^{2} \beta^{2} \sigma_{\zeta}^{2} \zeta^{2}- \\
& -4 \rho_{t} \beta^{3} \sigma_{\zeta}^{3} \zeta^{3}+\beta^{4} \sigma_{\zeta}^{4} \zeta^{4} .
\end{aligned}
$$


Utilizando as expansões acima podemos escrever (F.38) como

$$
p\left(y_{t} \mid x_{t}\right) \cong \frac{e^{-\frac{1}{2}\left(\frac{\rho_{t}-\beta \sigma_{\zeta} \zeta}{\sigma_{\varepsilon}}\right)^{2}}}{\sqrt{2 \pi} \sigma_{\varepsilon}}\left\{1+a_{0}+a_{1} \zeta+a_{2} \zeta^{2}+a_{3} \zeta^{3}+a_{4} \zeta^{4}\right\},
$$

sendo que os termos $a_{i}$ são dados por

$$
\begin{aligned}
& a_{0}=\frac{\gamma_{1, \varepsilon}}{6}\left[\left(\frac{\rho_{t}}{\sigma_{\varepsilon}}\right)^{3}-3\left(\frac{\rho_{t}}{\sigma_{\varepsilon}}\right)\right]+\frac{\gamma_{2, \varepsilon}}{24}\left[\left(\frac{\rho_{t}}{\sigma_{\varepsilon}}\right)^{4}-6\left(\frac{\rho_{t}}{\sigma_{\varepsilon}}\right)^{2}+3\right], \\
& a_{1}=\frac{\gamma_{1, \varepsilon}}{6}\left[\frac{-3 \rho_{t}^{2} \beta \sigma_{\zeta}}{\sigma_{\varepsilon}^{3}}-3\left(\frac{-\beta \sigma_{\zeta}}{\sigma_{\varepsilon}}\right)\right]+ \\
& +\frac{\gamma_{2, \varepsilon}}{24}\left[\frac{-4 \rho_{t}^{3} \beta \sigma_{\zeta}}{\sigma_{\varepsilon}^{4}}-6 \frac{\left(-2 \rho_{t} \beta \sigma_{\zeta}\right)}{\sigma_{\varepsilon}^{2}}\right] \\
& a_{1}=-\frac{\gamma_{1, \varepsilon}}{2} \frac{\beta \sigma_{\zeta}}{\sigma_{\varepsilon}}\left[\left(\frac{\rho_{t}}{\sigma_{\varepsilon}}\right)^{2}-1\right]-\frac{\gamma_{2, \varepsilon}}{6} \frac{\beta \sigma_{\zeta}}{\sigma_{\varepsilon}}\left[\left(\frac{\rho_{t}}{\sigma_{\varepsilon}}\right)^{3}-3\left(\frac{\rho_{t}}{\sigma_{\varepsilon}}\right)\right] \text {, } \\
& a_{2}=\frac{\gamma_{1, \varepsilon}}{6}\left[\frac{3 \rho_{t} \beta^{2} \sigma_{\zeta}^{2}}{\sigma_{\varepsilon}^{3}}\right]+\frac{\gamma_{2, \varepsilon}}{24}\left[\frac{6 \rho_{t}^{2} \beta^{2} \sigma_{\zeta}^{2}}{\sigma_{\varepsilon}^{4}}-6 \frac{\beta^{2} \sigma_{\zeta}^{2}}{\sigma_{\varepsilon}^{2}}\right] \\
& a_{2}=\frac{\gamma_{1, \varepsilon}}{2} \frac{\beta^{2} \sigma_{\zeta}^{2}}{\sigma_{\varepsilon}^{2}}\left(\frac{\rho_{t}}{\sigma_{\varepsilon}}\right)+\frac{\gamma_{2, \varepsilon}}{4} \frac{\beta^{2} \sigma_{\zeta}^{2}}{\sigma_{\varepsilon}^{2}}\left[\left(\frac{\rho_{t}}{\sigma_{\varepsilon}}\right)^{2}-1\right] \text {, } \\
& a_{3}=\frac{\gamma_{1, \varepsilon}}{6}\left[\frac{-\beta^{3} \sigma_{\zeta}^{3}}{\sigma_{\varepsilon}^{3}}\right]+\frac{\gamma_{2, \varepsilon}}{24}\left[\frac{-4 \rho_{t} \beta^{3} \sigma_{\zeta}^{3}}{\sigma_{\varepsilon}^{4}}\right] \\
& a_{3}=-\frac{\gamma_{1, \varepsilon}}{6} \frac{\beta^{3} \sigma_{\zeta}^{3}}{\sigma_{\varepsilon}^{3}}-\frac{\gamma_{2, \varepsilon}}{6} \frac{\beta^{3} \sigma_{\zeta}^{3}}{\sigma_{\varepsilon}^{3}}\left(\frac{\rho_{t}}{\sigma_{\varepsilon}}\right) \text {, } \\
& a_{4}=\frac{\gamma_{2, \varepsilon}}{24} \frac{\beta^{4} \sigma_{\zeta}^{4}}{\sigma_{\varepsilon}^{4}} .
\end{aligned}
$$

Podemos agora obter a distribuição conjunta de $x_{t}, y_{t} \mid y_{1: t-1}$ através de (F.33), (F.27) e 


\section{(F.42) por}

$$
\begin{aligned}
p\left(x_{t}, y_{t} \mid y_{1: t-1}\right)= & p\left(y_{t} \mid x_{t}\right) p\left(x_{t} \mid y_{1: t-1}\right) \\
\cong & \frac{e^{-\frac{1}{2}\left(\frac{\rho_{t}-\beta \sigma_{\zeta} \zeta}{\sigma_{\varepsilon}}\right)^{2}}}{\sqrt{2 \pi} \sigma_{\varepsilon}}\left\{1+a_{0}+a_{1} \zeta+a_{2} \zeta^{2}+a_{3} \zeta^{3}+a_{4} \zeta^{4}\right\} \\
& \cdot \frac{e^{-\frac{1}{2} \zeta^{2}}}{\sqrt{2 \pi} \sigma_{t}^{t-1}}\left\{1+\frac{\gamma_{1, t}^{t-1}}{6}\left(\zeta^{3}-3 \zeta\right)+\frac{\gamma_{2, t}^{t-1}}{24}\left(\zeta^{4}-6 \zeta^{2}+3\right)\right\} \\
= & \frac{1}{2 \pi \sigma_{\varepsilon} \sigma_{\zeta}} \exp \left\{-\frac{1}{2}\left[\left(\frac{\rho_{t}-\beta \sigma_{\zeta} \zeta}{\sigma_{\varepsilon}}\right)^{2}+\zeta^{2}\right]\right\}\{\cdots\} \\
= & \frac{1}{2 \pi \sigma_{\varepsilon} \sigma_{\zeta}} \exp \left\{-\frac{1}{2}\left[\frac{\rho_{t}^{2}-2 \rho_{t} \beta \sigma_{\zeta} \zeta+\beta^{2} \sigma_{\zeta}^{2} \zeta^{2}}{\sigma_{\varepsilon}^{2}}+\zeta^{2}\right]\right\}\{\cdots\} \\
= & \frac{1}{2 \pi \sigma_{\varepsilon} \sigma_{\zeta}} \exp \left\{-\frac{1}{2}\left(d \zeta^{2}-2 e \zeta+f\right)\right\}\{\cdots\} \\
= & \frac{1}{2 \pi \sigma_{\varepsilon} \sigma_{\zeta}} \exp \left\{-\frac{1}{2} d\left[\zeta^{2}-2 \frac{e}{d} \zeta+\frac{f}{d}\right]\right\}\{\cdots\} \\
= & \frac{1}{2 \pi \sigma_{\varepsilon} \sigma_{\zeta}} \exp \left\{-\frac{1}{2} d\left[\left(\zeta-\frac{e}{d}\right)^{2}-\left(\frac{e}{d}\right)^{2}+\frac{f}{d}\right]\right\}\{\cdots\} \\
= & \frac{1}{2 \pi \sigma_{\varepsilon} \sigma_{\zeta}} \exp \left\{-\frac{1}{2}\left[d\left(\zeta-\frac{e}{d}\right)^{2}+f-\frac{e^{2}}{d}\right]\right\}\{\cdots\},
\end{aligned}
$$

onde

$$
\begin{gathered}
d=\frac{\beta^{2} \sigma_{\zeta}^{2}+\sigma_{\varepsilon}^{2}}{\sigma_{\varepsilon}^{2}}, \\
e=\frac{\rho_{t} \beta \sigma_{\zeta}}{\sigma_{\varepsilon}^{2}}, \\
f=\frac{\rho_{t}^{2}}{\sigma_{\varepsilon}^{2}}
\end{gathered}
$$

e

$$
\begin{aligned}
\{\cdots\}= & \left\{1+a_{0}+a_{1} \zeta+a_{2} \zeta^{2}+a_{3} \zeta^{3}+a_{4} \zeta^{4}\right\} \\
& \cdot\left\{1+\frac{\gamma_{1, t}^{t-1}}{6}\left(\zeta^{3}-3 \zeta\right)+\frac{\gamma_{2, t}^{t-1}}{24}\left(\zeta^{4}-6 \zeta^{2}+3\right)\right\} \\
= & 1+\sum_{i=0}^{8} b_{i} \zeta^{i} .
\end{aligned}
$$

Por sua vez, os coeficientes $b_{i}$ são dados por

$$
b_{0}=\frac{\gamma_{2, t}^{t-1}}{8}+a_{0}\left(1+\frac{\gamma_{2, t}^{t-1}}{8}\right)
$$




$$
\begin{gathered}
b_{1}=-\left(1+a_{0}\right) \frac{\gamma_{1, t}^{t-1}}{2}+a_{1}\left(1+\frac{\gamma_{2, t}^{t-1}}{8}\right) \\
b_{2}=-\left(1+a_{0}\right) \frac{\gamma_{2, t}^{t-1}}{4}-a_{1} \frac{\gamma_{1, t}^{t-1}}{2}+a_{2}\left(1+\frac{\gamma_{2, t}^{t-1}}{8}\right) \\
b_{3}=\left(1+a_{0}\right) \frac{\gamma_{1, t}^{t-1}}{6}-a_{1} \frac{\gamma_{2, t}^{t-1}}{4}-a_{2} \frac{\gamma_{1, t}^{t-1}}{2}+a_{3}\left(1+\frac{\gamma_{2, t}^{t-1}}{8}\right), \\
b_{4}=\left(1+a_{0}\right) \frac{\gamma_{2, t}^{t-1}}{24}+a_{1} \frac{\gamma_{1, t}^{t-1}}{6}-a_{2} \frac{\gamma_{2, t}^{t-1}}{4}-a_{3} \frac{\gamma_{1, t}^{t-1}}{2}+a_{4}\left(1+\frac{\gamma_{2, t}^{t-1}}{8}\right), \\
b_{5}=a_{1} \frac{\gamma_{2, t}^{t-1}}{24}+a_{2} \frac{\gamma_{1, t}^{t-1}}{6}-a_{3} \frac{\gamma_{2, t}^{t-1}}{4}-a_{4} \frac{\gamma_{1, t}^{t-1}}{2}, \\
b_{6}=a_{2} \frac{\gamma_{2, t}^{t-1}}{24}+a_{3} \frac{\gamma_{1, t}^{t-1}}{6}-a_{4} \frac{\gamma_{2, t}^{t-1}}{4}, \\
b_{7}=a_{3} \frac{\gamma_{2, t}^{t-1}}{24}+a_{4} \frac{\gamma_{1, t}^{t-1}}{6}, \\
b_{8}=a_{4} \frac{\gamma_{2, t}^{t-1}}{24} .
\end{gathered}
$$

Podemos simplificar (F.48) se notarmos que

$$
\begin{aligned}
\frac{e}{d} & =\frac{\rho_{t} \beta \sigma_{\zeta}}{\beta^{2} \sigma_{\zeta}^{2}+\sigma_{\varepsilon}^{2}}, \\
f-\frac{e^{2}}{d} & =\frac{\rho_{t}^{2}}{\sigma_{\varepsilon}^{2}}-\frac{\rho_{t} \beta^{2} \sigma_{\zeta}^{2}}{\left(\beta^{2} \sigma_{\zeta}^{2}+\sigma_{\varepsilon}^{2}\right) \sigma_{\varepsilon}^{2}} \\
& =\frac{\rho_{t}^{2}}{\beta^{2} \sigma_{\zeta}^{2}+\sigma_{\varepsilon}^{2}} .
\end{aligned}
$$

Utilizando as equações acima em (F.48) obtemos

$$
\begin{aligned}
p\left(x_{t}, y_{t} \mid y_{1: t-1}\right) & \cong \frac{1}{2 \pi \sigma_{\varepsilon} \sigma_{\zeta}} \exp \left\{-\frac{1}{2}\left[d\left(\zeta-\frac{e}{d}\right)^{2}+f-\frac{e^{2}}{d}\right]\right\}\left\{1+\sum_{i=0}^{8} b_{i} \zeta^{i}\right\} \\
& =\frac{e^{-\frac{1}{2} \frac{\rho_{t}^{2}}{\beta^{2} \sigma_{\zeta}^{2}+\sigma_{\varepsilon}^{2}}}}{\sqrt{2 \pi} \sigma_{\varepsilon} \sigma_{\zeta} \sqrt{d}} \frac{e^{-\frac{1}{2}\left(\frac{\zeta-\frac{e}{d}}{1 / \sqrt{d}}\right)^{2}}}{\sqrt{2 \pi}(1 / \sqrt{d})}\left\{1+\sum_{i=0}^{8} b_{i} \zeta^{i}\right\} .
\end{aligned}
$$

A distribuição marginal de $y_{t} \mid y_{1: t-1}$ pode ser obtida por

$$
p\left(y_{t} \mid y_{1: t-1}\right)=\int_{-\infty}^{\infty} p\left(x_{t}, y_{t} \mid y_{1: t-1}\right) d x_{t} .
$$


Para efetuar a integral acima lembremos que

$$
\zeta=\frac{x_{t}-\mu_{\zeta}}{\sigma_{\zeta}} \Rightarrow d \zeta=\frac{d x_{t}}{\sigma_{\zeta}}
$$

e portanto

$$
p\left(y_{t} \mid y_{1: t-1}\right)=\int_{-\infty}^{\infty} p\left(\mu_{\zeta}+\sigma_{\zeta} \zeta, y_{t} \mid y_{1: t-1}\right) \sigma_{\zeta} d \zeta .
$$

Logo podemos escrever

$$
p\left(y_{t} \mid y_{1: t-1}\right) \cong \frac{e^{-\frac{1}{2} \frac{\rho_{t}^{2}}{\beta^{2} \sigma_{\zeta}^{2}+\sigma_{\varepsilon}^{2}}}}{\sqrt{2 \pi} \sigma_{\varepsilon} \sqrt{d}} \int_{-\infty}^{\infty} \frac{e^{-\frac{1}{2}\left(\frac{\zeta-\frac{e}{d}}{1 / \sqrt{d}}\right)^{2}}}{\sqrt{2 \pi}(1 / \sqrt{d})}\left\{1+\sum_{i=0}^{8} b_{i} \zeta^{i}\right\} d \zeta .
$$

Note agora que o núcleo da integral em (F.63) é uma densidade normal, com média $e / d$ e desvio padrão $1 / \sqrt{d}, \zeta \sim N\left(\mu_{d}, \sigma_{d}^{2}\right)$, onde

$$
\begin{gathered}
\mu_{d}=\frac{e}{d}=\frac{\rho_{t} \beta \sigma_{\zeta}}{\beta^{2} \sigma_{\zeta}^{2}+\sigma_{\varepsilon}^{2}}, \\
\sigma_{d}=\frac{1}{\sqrt{d}}=\frac{\sigma_{\varepsilon}}{\sqrt{\beta^{2} \sigma_{\zeta}^{2}+\sigma_{\varepsilon}^{2}}} .
\end{gathered}
$$

O $j$-ésimo momento não central, $m_{j}^{\prime}$, da distribuição $N\left(\mu_{d}, \sigma_{d}\right)$ é dado por

$$
\begin{aligned}
m_{j}^{\prime} & =\int_{-\infty}^{\infty} \frac{e^{-\frac{1}{2}\left(\frac{\zeta-\frac{e}{d}}{1 / \sqrt{d}}\right)^{2}}}{\sqrt{2 \pi}(1 / \sqrt{d})} \zeta^{j} d \zeta \\
& =\int_{-\infty}^{\infty} \frac{e^{-\frac{1}{2}\left(\frac{\zeta-\mu_{d}}{\sigma_{d}}\right)^{2}}}{\sqrt{2 \pi} \sigma_{d}} \zeta^{j} d \zeta
\end{aligned}
$$

Os momentos não centrais $m_{j}^{\prime}$ podem ser obtidos pela relação $(\underline{\mathrm{F} .22})$, de onde obtemos:

$$
\begin{gathered}
m_{0}^{\prime}=1, \\
m_{1}^{\prime}=\mu_{d}, \\
m_{2}^{\prime}=\sigma_{d}^{2}+\mu_{d}^{2}, \\
m_{3}^{\prime}=3 \mu_{d} \sigma_{d}^{2}+\mu_{d}^{3}, \\
m_{4}^{\prime}=3 \sigma_{d}^{4}+6 \mu_{d}^{2} \sigma_{d}^{2}+\mu_{d}^{4},
\end{gathered}
$$




$$
\begin{gathered}
m_{5}^{\prime}=15 \mu_{d} \sigma_{d}^{4}+10 \mu_{d}^{3} \sigma_{d}^{2}+\mu_{d}^{5}, \\
m_{6}^{\prime}=15 \sigma_{d}^{6}+45 \mu_{d}^{2} \sigma_{d}^{4}+15 \mu_{d}^{4} \sigma_{d}^{2}+\mu_{d}^{6}, \\
m_{7}^{\prime}=105 \mu_{d} \sigma_{d}^{6}+105 \mu_{d}^{3} \sigma_{d}^{4}+21 \mu_{d}^{5} \sigma_{d}^{2}+\mu_{d}^{7}, \\
m_{8}^{\prime}=105 \sigma_{d}^{8}+420 \mu_{d}^{2} \sigma_{d}^{6}+210 \mu_{d}^{4} \sigma_{d}^{4}+28 \mu_{d}^{6} \sigma_{d}^{2}+\mu_{d}^{8}, \\
m_{9}^{\prime}=945 \mu_{d} \sigma_{d}^{8}+1260 \mu_{d}^{3} \sigma_{d}^{6}+378 \mu_{d}^{5} \sigma_{d}^{4}+36 \mu_{d}^{7} \sigma_{d}^{2}+\mu_{d}^{9}, \\
m_{10}^{\prime}=945 \sigma_{d}^{10}+4725 \mu_{d}^{2} \sigma_{d}^{8}+3150 \mu_{d}^{4} \sigma_{d}^{6}+ \\
+630 \mu_{d}^{6} \sigma_{d}^{4}+45 \mu_{d}^{8} \sigma_{d}^{2}+\mu_{d}^{10}, \\
m_{12}^{\prime}={ }_{11}^{\prime}=10395 \sigma_{d}^{12}+62370 \mu_{d}^{2} \sigma_{d}^{10}+51975 \mu_{d}^{4} \sigma_{d}^{8}+ \\
+13860 \mu_{d}^{6} \sigma_{d}^{6}+1485 \mu_{d}^{8} \sigma_{d}^{4}+66 \mu_{d}^{10} \sigma_{d}^{2}+\mu_{d}^{12} . \\
+990 \mu_{d}^{7} \sigma_{d}^{4}+55 \mu_{d}^{9} \sigma_{d}^{2}+\mu_{d}^{11}, \\
\\
m_{d}^{\prime} \sigma_{d}^{10}+17325 \mu_{d}^{3} \sigma_{d}^{8}+6930 \mu_{d}^{5} \sigma_{d}^{6}+
\end{gathered}
$$

No que segue é conveniente utilizar a seguinte notação:

$$
g_{k}=\int_{-\infty}^{\infty} \frac{e^{-\frac{1}{2}\left(\frac{\zeta-\frac{e}{d}}{1 / \sqrt{d}}\right)^{2}}}{\sqrt{2 \pi}(1 / \sqrt{d})}\left\{1+b_{0}+\sum_{i=1}^{8} b_{i} \zeta^{i}\right\} \zeta^{k} d \zeta
$$

Note que podemos escrever (․ㅛ em função dos momentos não centrais $m_{j}^{\prime}$ da seguinte forma:

$$
\begin{aligned}
g_{k} & =\int_{-\infty}^{\infty} \frac{e^{-\frac{1}{2}\left(\frac{\zeta-\mu_{d}}{\sigma_{d}}\right)^{2}}}{\sqrt{2 \pi} \sigma_{d}}\left\{1+b_{0}+\sum_{i=1}^{8} b_{i} \zeta^{i}\right\} \zeta^{k} d \zeta \\
& =\int_{-\infty}^{\infty} \frac{e^{-\frac{1}{2}\left(\frac{\zeta-\mu_{d}}{\sigma_{d}}\right)^{2}}}{\sqrt{2 \pi} \sigma_{d}}\left\{\left(1+b_{0}\right) \zeta^{k}+\sum_{i=1}^{8} b_{i} \zeta^{k+i}\right\} d \zeta
\end{aligned}
$$




$$
g_{k}=\left(1+b_{0}\right) m_{k}^{\prime}+\sum_{i=1}^{8} b_{i} m_{k+i}^{\prime} .
$$

Por exemplo, utilizando ( (F.79) por

$$
\begin{gathered}
g_{0}=\left(1+b_{0}\right) m_{0}^{\prime}+\sum_{i=1}^{8} b_{i} m_{i}^{\prime}, \\
g_{0}=1+b_{0}+\sum_{i=1}^{8} b_{i} m_{i}^{\prime}, \\
g_{1}=m_{1}^{\prime}+\sum_{i=1}^{8} b_{i} m_{1+i}^{\prime}, \\
g_{1}=\mu_{d}+\sum_{i=1}^{8} b_{i} m_{1+i}^{\prime}, \\
g_{2}=m_{2}^{\prime}+\sum_{i=1}^{8} b_{i} m_{2+i}^{\prime}, \\
g_{2}=\sigma_{d}^{2}+\mu_{d}^{2}+\sum_{i=1}^{8} b_{i} m_{2+i}^{\prime} .
\end{gathered}
$$

Assim, utilizando (F.78) podemos escrever a distribuição de $y_{t} \mid y_{1: t-1}$ como

$$
p\left(y_{t} \mid y_{1: t-1}\right) \cong \frac{e^{-\frac{1}{2} \frac{\rho_{t}^{2}}{\beta^{2} \sigma_{\zeta}^{2}+\sigma_{\varepsilon}^{2}}}}{\sqrt{2 \pi} \sqrt{\beta^{2} \sigma_{\zeta}^{2}+\sigma_{\varepsilon}^{2}}} g_{0} .
$$

Podemos finalmente obter a distribuição de $x_{t} \mid y_{1: t}$ através de

$$
p\left(x_{t} \mid y_{1: t}\right)=\frac{p\left(x_{t}, y_{t} \mid y_{1: t-1}\right)}{p\left(y_{t} \mid y_{1: t-1}\right)} .
$$

Utilizando (

$$
\begin{aligned}
& p\left(x_{t} \mid y_{1: t}\right) \cong \frac{e^{-\frac{1}{2} \frac{\rho_{t}^{2}}{\beta^{2} \sigma_{\zeta}^{2}+\sigma_{\varepsilon}^{2}}}}{\sqrt{2 \pi} \sigma_{\varepsilon} \sigma_{\zeta} \sqrt{d}} \frac{e^{-\frac{1}{2}\left(\frac{\zeta-\mu_{d}}{\sigma_{d}}\right)^{2}}}{\sqrt{2 \pi} \sigma_{d}}\left\{1+\sum_{i=0}^{8} b_{i} \zeta^{i}\right\}\left[\frac{e^{-\frac{1}{2} \frac{\rho_{t}^{2}}{\beta^{2} \sigma_{\zeta}^{2}+\sigma_{\varepsilon}^{2}}}}{\sqrt{2 \pi} \sigma_{\varepsilon} \sqrt{d}} g_{0}\right]^{-1} \\
& p\left(x_{t} \mid y_{1: t}\right) \cong \frac{e^{-\frac{1}{2}\left(\frac{\zeta-\mu_{d}}{\sigma_{d}}\right)^{2}}}{\sqrt{2 \pi} \sigma_{\zeta} \sigma_{d} g_{0}}\left\{1+\sum_{i=0}^{8} b_{i} \zeta^{i}\right\} .
\end{aligned}
$$

Para que possamos desenvolver um procedimento recursivo para atualizar as estimativas para as variáveis latentes, precisamos aproximar a distribuição de $x_{t} \mid y_{1: t}$ através da expansão de Gram-Charlier. Para isso, vamos obter inicialmente os momentos não centrais $\mu_{r}^{\prime}=$ 
$E\left[X_{t}^{r} \mid y_{1: t}\right]^{2}:$

$$
\begin{aligned}
\mu_{r}^{\prime} & =E\left[X_{t}^{r} \mid y_{1: t}\right] \\
& =\int_{-\infty}^{\infty} p\left(x_{t} \mid y_{1: t}\right) x_{t}^{r} d x_{t} \\
& =\int_{-\infty}^{\infty} p\left(\mu_{\zeta}+\sigma_{\zeta} \zeta \mid y_{1: t}\right)\left(\mu_{\zeta}+\sigma_{\zeta} \zeta\right)^{r} \sigma_{\zeta} d \zeta \\
& =\int_{-\infty}^{\infty} p\left(\mu_{\zeta}+\sigma_{\zeta} \zeta \mid y_{1: t}\right) \sum_{j=0}^{r}\left(\begin{array}{l}
r \\
j
\end{array}\right) \sigma_{\zeta}^{j} \zeta^{j} \mu_{\zeta}^{r-j} \sigma_{\zeta} d \zeta \\
& \cong \int_{-\infty}^{\infty} \frac{e^{-\frac{1}{2}\left(\frac{\zeta-\mu_{d}}{\sigma_{d}}\right)^{2}}}{\sqrt{2 \pi} \sigma_{\zeta} \sigma_{d} g_{0}}\left\{1+\sum_{i=0}^{8} b_{i} \zeta^{i}\right\} \sum_{j=0}^{r}\left(\begin{array}{l}
r \\
j
\end{array}\right) \sigma_{\zeta}^{j+1} \zeta^{j} \mu_{\zeta}^{r-j} d \zeta \\
& =\sum_{j=0}^{r}\left(\begin{array}{l}
r \\
j
\end{array}\right) \frac{\sigma_{\zeta}^{j} \mu_{\zeta}^{r-j}}{g_{0}} \int_{-\infty}^{\infty} \frac{e^{-\frac{1}{2}\left(\frac{\zeta-\mu_{d}}{\sigma_{d}}\right)^{2}}}{\sqrt{2 \pi} \sigma_{\zeta} \sigma_{d}}\left\{\zeta^{j}+\sum_{i=0}^{8} b_{i} \zeta^{i+j}\right\} d \zeta \\
& =\sum_{j=0}^{r}\left(\begin{array}{l}
r \\
j
\end{array}\right) \frac{\sigma_{\zeta}^{j} \mu_{\zeta}^{r-j}}{g_{0}}\left(m_{j}^{\prime}+\sum_{i=0}^{8} b_{i} m_{i+j}^{\prime}\right) \\
& =\sum_{j=0}^{r}\left(\begin{array}{l}
r \\
j
\end{array}\right) \frac{\sigma_{\zeta}^{j} \mu_{\zeta}^{r-j}}{g_{0}}\left(\left(1+b_{0}\right) m_{j}^{\prime}+\sum_{i=1}^{8} b_{i} m_{i+j}^{\prime}\right) \\
& =\sum_{j=0}^{r}\left(\begin{array}{l}
r \\
j
\end{array}\right) \frac{\sigma_{\zeta}^{j} \mu_{\zeta}^{r-j}}{g_{0}} g_{j} .
\end{aligned}
$$

Logo

$$
\mu_{r}^{\prime} \cong \frac{1}{g_{0}} \sum_{j=0}^{r}\left(\begin{array}{l}
r \\
j
\end{array}\right) \sigma_{\zeta}^{j} \mu_{\zeta}^{r-j} g_{j}
$$

Temos assim,

$$
\begin{gathered}
\mu_{1}^{\prime} \cong \mu_{\zeta}+\sigma_{\zeta} \frac{g_{1}}{g_{0}} \\
\mu_{2}^{\prime} \cong \mu_{\zeta}^{2}+2 \mu_{\zeta} \sigma_{\zeta} \frac{g_{1}}{g_{0}}+\sigma_{\zeta}^{2} \frac{g_{2}}{g_{0}} \\
\mu_{3}^{\prime} \cong \mu_{\zeta}^{3}+3 \mu_{\zeta}^{2} \sigma_{\zeta} \frac{g_{1}}{g_{0}}+3 \mu_{\zeta} \sigma_{\zeta}^{2} \frac{g_{2}}{g_{0}}+\sigma_{\zeta}^{3} \frac{g_{3}}{g_{0}} \\
\mu_{4}^{\prime} \cong \mu_{\zeta}^{4}+4 \mu_{\zeta}^{3} \sigma_{\zeta} \frac{g_{1}}{g_{0}}+6 \mu_{\zeta}^{2} \sigma_{\zeta}^{2} \frac{g_{2}}{g_{0}}+4 \mu_{\zeta} \sigma_{\zeta}^{3} \frac{g_{3}}{g_{0}}+\sigma_{\zeta}^{4} \frac{g_{4}}{g_{0}}
\end{gathered}
$$

Finalmente, podemos obter os momentos centrais da distribuição de $x_{t} \mid y_{1: t}, \quad \mu_{i}=$

\footnotetext{
${ }^{2}$ A dedução a seguir foi desenvolvida pelo Prof. Gauss Moutinho Cordeiro.
} 
$E\left[\left(X_{t}-E\left[X_{t} \mid y_{1: t}\right]\right)^{i} \mid y_{1: t}\right]$, por (veja por exemplo, Stuart e Ord (1994), pg. 75):

$$
\begin{aligned}
& \mu_{2}=\mu_{2}^{\prime}-\mu_{1}^{\prime 2}, \\
& \mu_{3}=\mu_{3}^{\prime}-3 \mu_{2}^{\prime} \mu_{1}^{\prime}+2 \mu_{1}^{\prime 3}, \\
& \mu_{4}=\mu_{4}^{\prime}-4 \mu_{3}^{\prime} \mu_{1}^{\prime}+6 \mu_{2}^{\prime} \mu_{1}^{\prime 2}-3 \mu_{1}^{\prime 4} .
\end{aligned}
$$

Utilizando agora a nomenclatura do algoritmo recursivo (F.5) a (F.8) escrevemos:

$$
\begin{gathered}
\mu_{t}^{t}=\mu_{1}^{\prime} \\
\cong \mu_{\zeta}+\sigma_{\zeta} \frac{g_{1}}{1+h}, \\
\mu_{t}^{t} \cong \mu_{t}^{t-1}+\sigma_{t}^{t-1} \frac{g_{1}}{g_{0}} \\
v_{t}^{t}=\left(\sigma_{t}^{t}\right)^{2}=\mu_{2} \\
=\mu_{2}^{\prime}-\mu_{1}^{\prime 2} \\
\cong \mu_{\zeta}^{2}+2 \mu_{\zeta} \sigma_{\zeta} \frac{g_{1}}{g_{0}}+\sigma_{\zeta}^{2} \frac{g_{2}}{g_{0}}-\left(\mu_{\zeta}+\sigma_{\zeta} \frac{g_{1}}{g_{0}}\right)^{2} \\
=\mu_{\zeta}^{2}+2 \mu_{\zeta} \sigma_{\zeta} \frac{g_{1}}{g_{0}}+\sigma_{\zeta}^{2} \frac{g_{2}}{g_{0}}-\mu_{\zeta}^{2}-2 \mu_{\zeta} \sigma_{\zeta} \frac{g_{1}}{g_{0}}-\sigma_{\zeta}^{2}\left(\frac{g_{1}}{g_{0}}\right)^{2} \\
=\sigma_{\zeta}^{2}\left[\frac{g_{2}}{g_{0}}-\left(\frac{g_{1}}{g_{0}}\right)^{2}\right], \\
v_{t}^{t}=\left(\sigma_{t}^{t}\right)^{2} \cong v_{t}^{t-1}\left[\frac{g_{2}}{g_{0}}-\left(\frac{g_{1}}{g_{0}}\right)^{2}\right] \\
\gamma_{1, t}^{t}=\frac{\mu_{3}}{\left(\sigma_{t}^{t}\right)^{3}}, \\
\gamma_{2, t}^{t}=\frac{\mu_{4}}{\left(\sigma_{t}^{t}\right)^{4}}-3 .
\end{gathered}
$$




\section{Apêndice G}

\section{Estimativa das matrizes de covariância dos estimadores CECF e DECF}

Neste apêndice vamos apresentar os resultados das estimativas das matrizes de covariância dos estimadores CECF e DECF para o modelo SCD, para os processos simulados com parâmetros conhecidos $\delta=0.033, \phi=0.95, \sigma_{\eta}=0.1$ e $\lambda=2$ e 0.5 , aplicando para isso os resultados obtidos no capítulo 5 .

As tabelas G.1 a G.5 apresentam os resultados obtidos com o método CECF. As matrizes $A\left(\theta_{0}\right)$ foram estimadas através de $(5.10)$ e as matrizes $B\left(\theta_{0}\right)$ foram estimadas através de (5.18); a matriz $V=B\left(\theta_{0}\right)^{-1} A\left(\theta_{0}\right) B\left(\theta_{0}\right)^{-1}$, e a matriz de covariância do estimador é dada por $V / n$.

As tabelas G.6 a.10 apresentam os resultados obtidos com o método DECF. As matrizes $A\left(\theta_{0}\right)$ foram estimadas através de (5.41) e as matrizes $B\left(\theta_{0}\right)$ foram estimadas através de (5.40); a matriz $V=B\left(\theta_{0}\right)^{-1} A\left(\theta_{0}\right) B\left(\theta_{0}\right)^{-1}$, e a matriz de covariância do estimador é dada por $V / n$. 
Tabela G.1: Estimativa da matriz de covariância do modelo SCD com distribuição exponencial de parâmetros $\delta=0.033, \phi=0.95, \sigma_{\eta}=0.1$, através do método CECF. Veja o texto para mais detalhes.

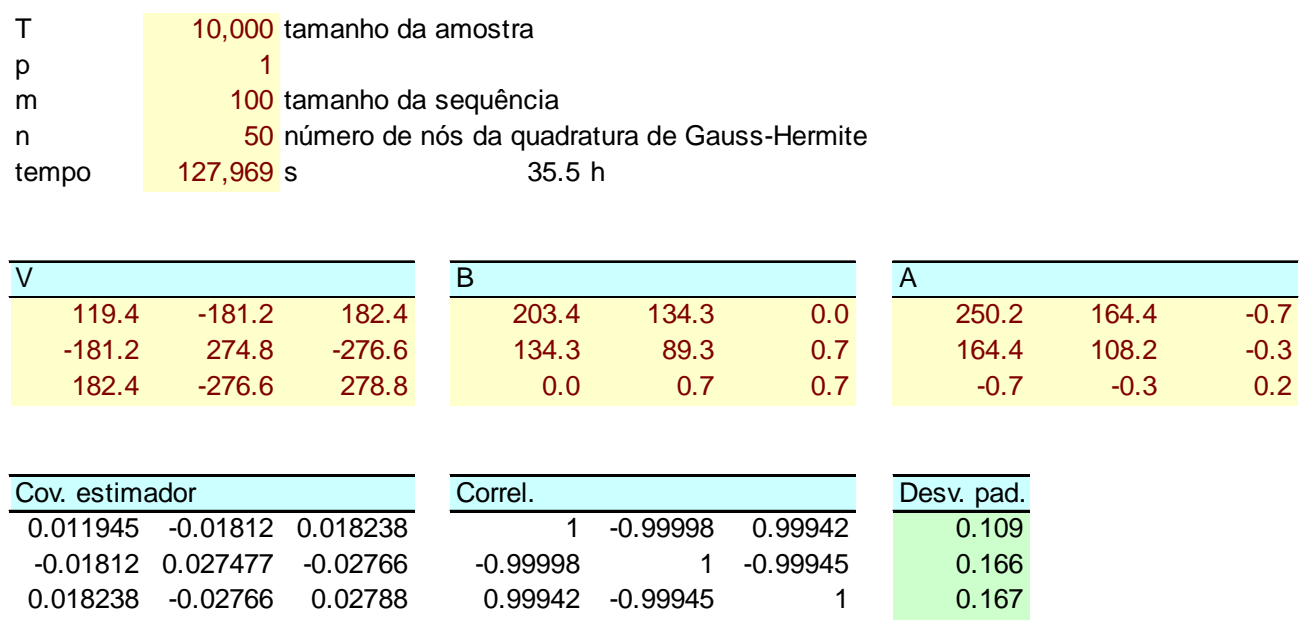

Tabela G.2: Estimativa da matriz de covariância do modelo SCD com distribuição Weibull de parâmetros $\delta=0.033, \phi=0.95, \sigma_{\eta}=0.1$ e $\lambda=2$, através do método CECF. Veja o texto para mais detalhes.

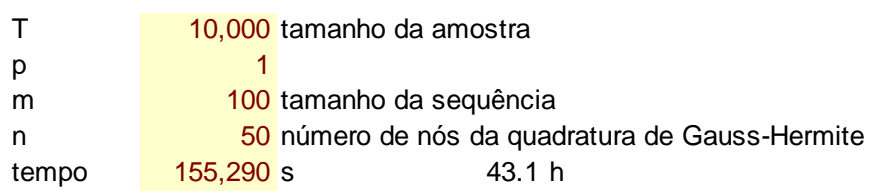

\begin{tabular}{rrrr}
\hline $\mathrm{V}$ & & & \\
\hline 694.5 & $-1,053.8$ & $1,060.9$ & 9.5 \\
$-1,053.8$ & $1,598.9$ & $-1,609.7$ & -14.5 \\
$1,060.9$ & $-1,609.7$ & $1,621.7$ & 14.9 \\
9.5 & -14.5 & 14.9 & 0.5
\end{tabular}

\begin{tabular}{llll}
\hline \multicolumn{4}{l}{ Cov. estimador } \\
\hline 0.069452 & -0.10538 & 0.106086 & 0.000955 \\
-0.10538 & 0.159886 & -0.16097 & -0.00145 \\
0.106086 & -0.16097 & 0.162173 & 0.001487 \\
0.000955 & -0.00145 & 0.001487 & $4.57 \mathrm{E}-05$
\end{tabular}

\begin{tabular}{|c|c|c|c|}
\hline \multicolumn{4}{|l|}{ B } \\
\hline 70.8 & 46.7 & 0.0 & 2.2 \\
\hline 46.7 & 31.0 & 0.2 & 1.2 \\
\hline 0.0 & 0.2 & 0.2 & -0.3 \\
\hline 2.2 & 1.2 & -0.3 & 1.3 \\
\hline
\end{tabular}

\begin{tabular}{rrrr}
\hline Correl. & & & \\
\hline 1 & -0.99999 & 0.99960 & 0.53595 \\
-0.99999 & 1 & -0.99963 & -0.5364 \\
0.99960 & -0.99963 & 1 & 0.546186 \\
0.53595 & -0.5364 & 0.546186 & 1
\end{tabular}

\begin{tabular}{rrrrr}
\hline $\mathrm{A}$ & & & & \\
\hline & 43.9 & 28.8 & -0.2 & 1.9 \\
28.8 & 18.9 & -0.1 & 1.2 \\
& -0.2 & -0.1 & 0.0 & -0.1 \\
& 1.9 & 1.2 & -0.1 & 0.5
\end{tabular}

\begin{tabular}{r}
\hline Desv. pad. \\
\hline 0.264 \\
0.400 \\
0.403 \\
0.007
\end{tabular}


Tabela G.3: Estimativa da matriz de covariância do modelo SCD com distribuição gama de parâmetros $\delta=0.033, \phi=0.95, \sigma_{\eta}=0.1$ e $\lambda=2$, através do método CECF. Veja o texto para mais detalhes.

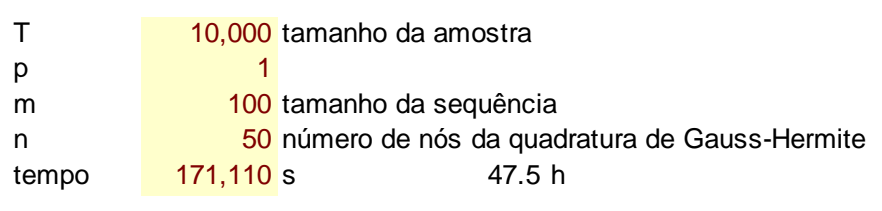

\begin{tabular}{rrrr}
\hline $\mathrm{V}$ & & & \\
\hline 91.0 & -138.2 & 141.0 & 40.6 \\
-138.2 & 209.9 & -214.2 & -61.7 \\
141.0 & -214.2 & 218.7 & 63.3 \\
40.6 & -61.7 & 63.3 & 23.9
\end{tabular}

\begin{tabular}{|c|c|c|c|}
\hline $\bar{B}$ & & & \\
\hline 410.4 & 270.8 & 0.0 & 1.8 \\
\hline 270.8 & 180.5 & 1.8 & 1.0 \\
\hline 0.0 & 1.8 & 1.8 & -0.2 \\
\hline 1.8 & 1.0 & -0.2 & 0.0 \\
\hline
\end{tabular}

\begin{tabular}{rrrrr}
\hline A & & & & \\
\hline & 831.5 & 547.7 & -1.1 & 3.8 \\
547.7 & 361.2 & -0.3 & 2.5 \\
& -1.1 & -0.3 & 0.4 & -0.1 \\
3.8 & 2.5 & -0.1 & 0.0
\end{tabular}

\begin{tabular}{rrrr}
\hline Cov. estimador & & \\
\hline 0.0091 & -0.01382 & 0.014104 & 0.004061 \\
-0.01382 & 0.020989 & -0.02142 & -0.00617 \\
0.014104 & -0.02142 & 0.021872 & 0.006327 \\
0.004061 & -0.00617 & 0.006327 & 0.00239
\end{tabular}

\begin{tabular}{lrrr}
\hline Correl. & & & \\
\hline 1 & -0.99997 & 0.99967 & 0.87070 \\
-0.99997 & 1 & -0.99970 & -0.87095 \\
0.99967 & -0.99970 & 1 & 0.875128 \\
0.87070 & -0.87095 & 0.875128 & 1
\end{tabular}

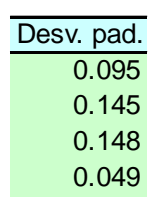

Tabela G.4: Estimativa da matriz de covariância do modelo SCD com distribuição Weibull de parâmetros $\delta=0.033, \phi=0.95, \sigma_{\eta}=0.1$ e $\lambda=0.5$, através do método CECF. Veja o texto para mais detalhes.

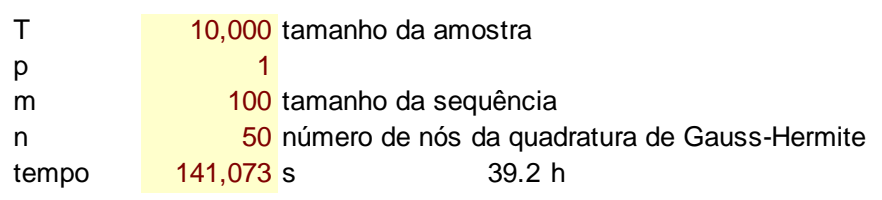

\begin{tabular}{rrrr}
\hline $\mathrm{V}$ & & & \\
\hline $4,221.9$ & $-6,408.9$ & $6,512.4$ & 35.0 \\
$-6,408.9$ & $9,729.0$ & $-9,886.3$ & -53.2 \\
$6,512.4$ & $-9,886.3$ & $10,050.6$ & 54.6 \\
35.0 & -53.2 & 54.6 & 0.5
\end{tabular}

\begin{tabular}{llll}
\hline \multicolumn{4}{l}{ Cov. estimador } \\
\hline 0.42219 & -0.64089 & 0.651243 & 0.003503 \\
-0.64089 & 0.972898 & -0.98863 & -0.00532 \\
0.651243 & -0.98863 & 1.005065 & 0.005462 \\
0.003503 & -0.00532 & 0.005462 & $4.79 \mathrm{E}-05$
\end{tabular}

\begin{tabular}{|c|c|c|c|}
\hline \multicolumn{4}{|l|}{$D$} \\
\hline 34.9 & 23.0 & 0.0 & 5.9 \\
\hline 23.0 & 15.3 & 0.1 & 3.6 \\
\hline 0.0 & 0.1 & 0.1 & -0.3 \\
\hline 5.9 & 3.6 & -0.3 & 3.3 \\
\hline \multicolumn{4}{|l|}{ Correl. } \\
\hline 1 & -1.00000 & 0.99975 & 0.77855 \\
\hline-1.00000 & 1 & -0.99977 & -0.77922 \\
\hline 0.99975 & -0.99977 & 1 & 0.786952 \\
\hline 0.77855 & -0.77922 & 0.786952 & 1 \\
\hline
\end{tabular}

\begin{tabular}{|c|c|c|c|}
\hline $\bar{A}$ & & & \\
\hline 15.4 & 10.1 & -0.1 & 2.9 \\
\hline 10.1 & 6.6 & 0.0 & 1.8 \\
\hline-0.1 & 0.0 & 0.0 & -0.1 \\
\hline 2.9 & 1.8 & -0.1 & 1.2 \\
\hline
\end{tabular}

\begin{tabular}{r}
\hline Desv. pad. \\
\hline 0.650 \\
0.986 \\
1.003 \\
0.007
\end{tabular}


Tabela G.5: Estimativa da matriz de covariância do modelo SCD com distribuição gama de parâmetros $\delta=0.033, \phi=0.95, \sigma_{\eta}=0.1$ e $\lambda=0.5$, através do método CECF. Veja o texto para mais detalhes.

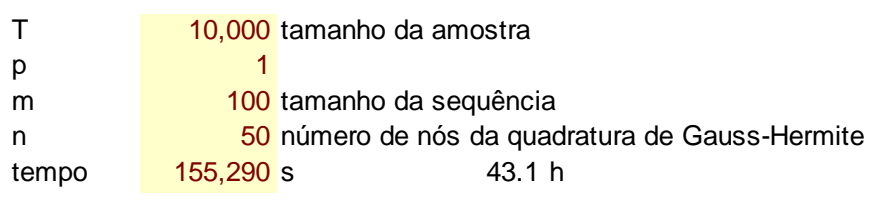

\begin{tabular}{rrrr}
\hline $\mathrm{V}$ & & & \\
\hline 694.5 & $-1,053.8$ & $1,060.9$ & 9.5 \\
$-1,053.8$ & $1,598.9$ & $-1,609.7$ & -14.5 \\
$1,060.9$ & $-1,609.7$ & $1,621.7$ & 14.9 \\
9.5 & -14.5 & 14.9 & 0.5
\end{tabular}

\begin{tabular}{rrrrr}
\hline B & & & & \\
\hline 70.8 & 46.7 & 0.0 & 2.2 \\
46.7 & 31.0 & 0.2 & 1.2 \\
& 0.0 & 0.2 & 0.2 & -0.3 \\
2.2 & 1.2 & -0.3 & 1.3
\end{tabular}

\begin{tabular}{rrrrr}
\hline A & & & & \\
\hline & 43.9 & 28.8 & -0.2 & 1.9 \\
28.8 & 18.9 & -0.1 & 1.2 \\
& -0.2 & -0.1 & 0.0 & -0.1 \\
1.9 & 1.2 & -0.1 & 0.5
\end{tabular}

\begin{tabular}{cccc}
\hline \multicolumn{4}{l}{ Cov. estimador } \\
\hline 0.069452 & -0.10538 & 0.106086 & 0.000955 \\
-0.10538 & 0.159886 & -0.16097 & -0.00145 \\
0.106086 & -0.16097 & 0.162173 & 0.001487 \\
0.000955 & -0.00145 & 0.001487 & $4.57 \mathrm{E}-05$
\end{tabular}

\begin{tabular}{lrrr}
\hline Correl. & & & \\
\hline 1 & -0.99999 & 0.99960 & 0.53595 \\
-0.99999 & 1 & -0.99963 & -0.5364 \\
0.99960 & -0.99963 & 1 & 0.546186 \\
0.53595 & -0.5364 & 0.546186 & 1
\end{tabular}

\begin{tabular}{r}
\hline Desv. pad. \\
\hline 0.264 \\
0.400 \\
0.403 \\
0.007
\end{tabular}

Tabela G.6: Estimativa da matriz de covariância do modelo SCD com distribuição exponencial de parâmetros $\delta=0.033, \phi=0.95, \sigma_{\eta}=0.1$, através do método DECF. Veja o texto para mais detalhes.
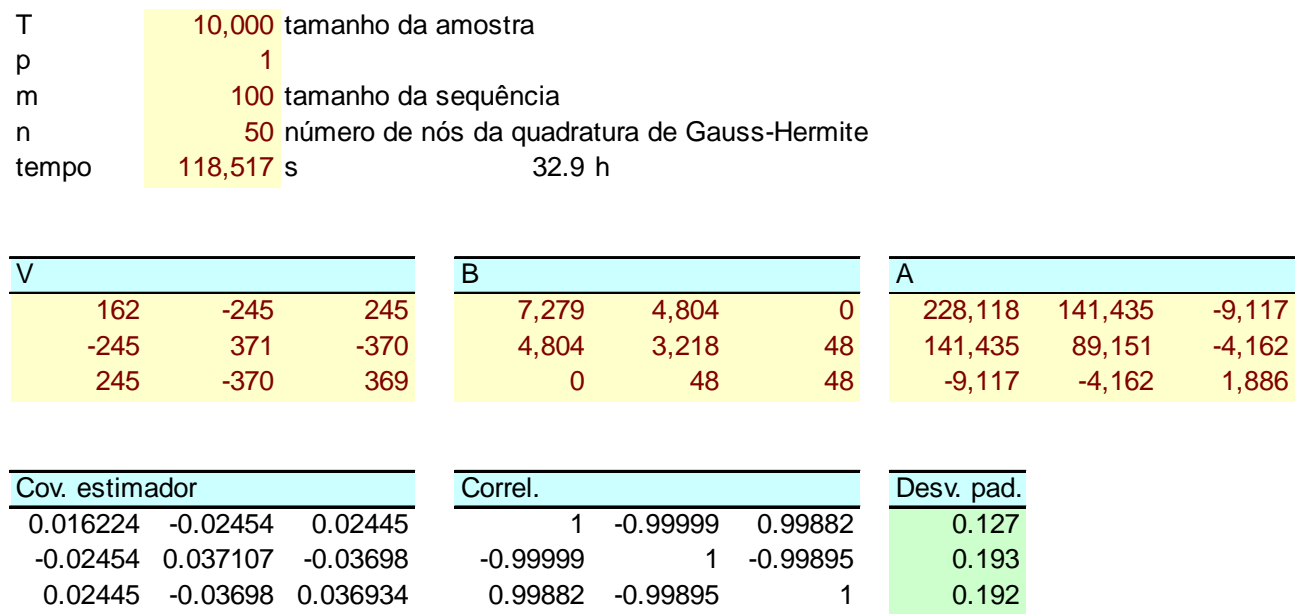

\begin{tabular}{rrr}
\hline Correl. & & \\
\hline 1 & -0.99999 & 0.99882 \\
-0.99999 & 1 & -0.99895 \\
0.99882 & -0.99895 & 1
\end{tabular}

Desv. pad.

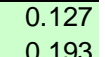

0.192 
Tabela G.7: Estimativa da matriz de covariância do modelo SCD com distribuição Weibull de parâmetros $\delta=0.033, \phi=0.95, \sigma_{\eta}=0.1$ e $\lambda=2$, através do método DECF. Veja o texto para mais detalhes.

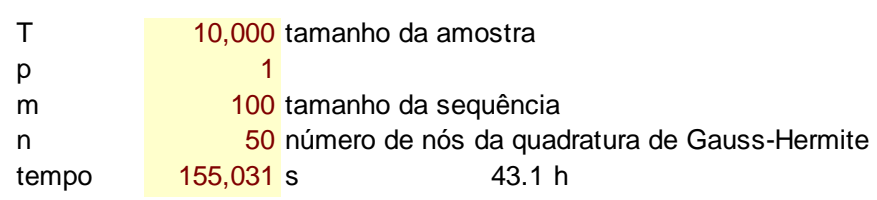

\begin{tabular}{|c|c|c|c|}
\hline $\bar{V}$ & & & \\
\hline 139 & -211 & 218 & 104 \\
\hline-211 & 322 & -332 & -158 \\
\hline 218 & -332 & 342 & 163 \\
\hline 104 & -158 & 163 & 81 \\
\hline
\end{tabular}

\begin{tabular}{|c|c|c|c|}
\hline $\mathrm{B}$ & & & \\
\hline 50,744 & 33,491 & 0 & 59 \\
\hline 33,491 & 22,744 & 648 & -28 \\
\hline 0 & 648 & 657 & -69 \\
\hline 59 & -28 & -69 & 13 \\
\hline
\end{tabular}

\begin{tabular}{lrrr}
\hline Correl. & & & \\
\hline 1 & -1.00000 & 0.99975 & 0.97805 \\
-1.00000 & 1 & -0.99979 & -0.97844 \\
0.99975 & -0.99979 & 1 & 0.981603 \\
0.97805 & -0.97844 & 0.981603 & 1
\end{tabular}

\begin{tabular}{rrrr}
\hline \multicolumn{1}{l}{ A } & & & \\
\hline $4,919,803$ & $3,290,464$ & 54,279 & $-40,694$ \\
$3,290,464$ & $2,234,698$ & 70,680 & $-31,017$ \\
54,279 & 70,680 & 35,395 & $-4,310$ \\
$-40,694$ & $-31,017$ & $-4,310$ & 815 \\
& & & \\
\hline Desv. pad. & & & \\
\hline 0.118 & & & \\
0.179 & & & \\
0.185 & & & \\
0.090 & & &
\end{tabular}

Tabela G.8: Estimativa da matriz de covariância do modelo SCD com distribuição gama de parâmetros $\delta=0.033, \phi=0.95, \sigma_{\eta}=0.1$ e $\lambda=2$, através do método DECF. Veja o texto para mais detalhes.

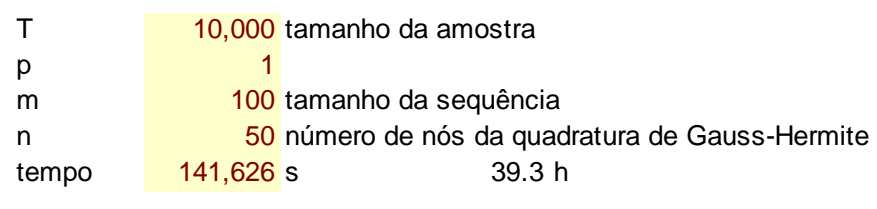

\begin{tabular}{rrrr}
\hline $\mathrm{V}$ & & & \\
\hline 322 & -489 & 498 & 221 \\
-489 & 743 & -757 & -336 \\
498 & -757 & 772 & 344 \\
221 & -336 & 344 & 164
\end{tabular}

\begin{tabular}{lrrr}
\hline \multicolumn{4}{l}{ Cov. estimador } \\
\hline 0.032219 & -0.04891 & 0.049846 & 0.022127 \\
-0.04891 & 0.074254 & -0.07567 & -0.0336 \\
0.049846 & -0.07567 & 0.077153 & 0.034374 \\
0.022127 & -0.0336 & 0.034374 & 0.016367
\end{tabular}

\begin{tabular}{|c|c|c|c|}
\hline \multicolumn{4}{|l|}{$B$} \\
\hline 25,115 & 16,576 & 0 & 34 \\
\hline 16,576 & 11,189 & 252 & -3 \\
\hline 0 & 252 & 255 & -26 \\
\hline 34 & -3 & -26 & 5 \\
\hline \multicolumn{4}{|l|}{ Correl. } \\
\hline 1 & -1.00000 & 0.99976 & 0.96356 \\
\hline-1.00000 & 1 & -0.99979 & -0.96395 \\
\hline 0.99976 & -0.99979 & 1 & 0.967315 \\
\hline 0.96356 & -0.96395 & 0.967315 & 1 \\
\hline
\end{tabular}

\begin{tabular}{rrrr}
\hline \multicolumn{1}{l}{ A } & & & \\
\hline 942,277 & 623,374 & 3,159 & $-6,353$ \\
623,374 & 432,931 & 22,963 & $-6,972$ \\
3,159 & 22,963 & 21,235 & $-2,845$ \\
$-6,353$ & $-6,972$ & $-2,845$ & 446 \\
& & & \\
\hline Desv. pad. & & & \\
\hline 0.179 & & & \\
0.272 & & & \\
0.278 & & & \\
0.128 & & &
\end{tabular}


Tabela G.9: Estimativa da matriz de covariância do modelo SCD com distribuição Weibull de parâmetros $\delta=0.033, \phi=0.95, \sigma_{\eta}=0.1$ e $\lambda=0.5$, através do método DECF. Veja o texto para mais detalhes.

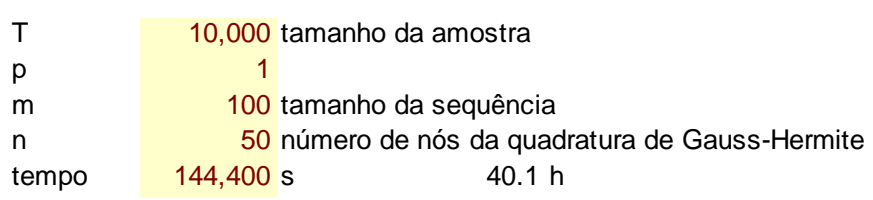

\begin{tabular}{rrrr}
\hline $\mathrm{V}$ & & & \\
\hline 12,879 & $-19,547$ & 19,755 & 89 \\
$-19,547$ & 29,669 & $-29,984$ & -135 \\
19,755 & $-29,984$ & 30,315 & 137 \\
89 & -135 & 137 & 1
\end{tabular}

\begin{tabular}{rrrr}
\hline \multicolumn{4}{l}{ Cov. estimador } \\
\hline 1.2879 & -1.95475 & 1.97554 & 0.008906 \\
-1.95475 & 2.966881 & -2.99845 & -0.01352 \\
1.97554 & -2.99845 & 3.031451 & 0.013745 \\
0.008906 & -0.01352 & 0.013745 & $9.13 E-05$
\end{tabular}

\begin{tabular}{|c|c|c|c|}
\hline $\mathrm{B}$ & & & \\
\hline 578 & 382 & 0 & 83 \\
\hline 382 & 253 & 1 & 49 \\
\hline 0 & 1 & 1 & -6 \\
\hline 83 & 49 & -6 & 56 \\
\hline
\end{tabular}

\begin{tabular}{lrrr}
\hline Correl. & & & \\
\hline 1 & -1.00000 & 0.99981 & 0.82140 \\
-1.00000 & 1 & -0.99982 & -0.8219 \\
0.99981 & -0.99982 & 1 & 0.826343 \\
0.82140 & -0.8219 & 0.826343 & 1
\end{tabular}

\begin{tabular}{rrrrr}
\hline $\mathrm{A}$ & & & & \\
\hline 6,271 & 4,206 & 66 & 539 \\
& 4,206 & 2,834 & 58 & 282 \\
& 66 & 58 & 15 & -76 \\
539 & 282 & -76 & 584
\end{tabular}

\begin{tabular}{r}
\hline Desv. pad. \\
\hline 1.135 \\
1.722 \\
1.741 \\
0.010
\end{tabular}

Tabela G.10: Estimativa da matriz de covariância do modelo SCD com distribuição gama de parâmetros $\delta=0.033, \phi=0.95, \sigma_{\eta}=0.1$ e $\lambda=0.5$, através do método CECF. Veja o texto para mais detalhes.

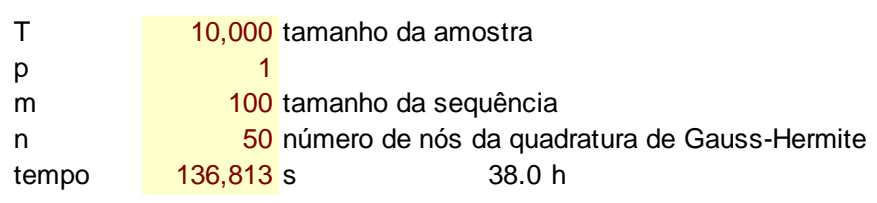

\begin{tabular}{rrrr}
\hline $\mathrm{V}$ & & & \\
\hline 1,048 & $-1,589$ & 1,599 & 17 \\
$-1,589$ & 2,409 & $-2,423$ & -25 \\
1,599 & $-2,423$ & 2,441 & 26 \\
17 & -25 & 26 & 1
\end{tabular}

\begin{tabular}{llll}
\hline \multicolumn{2}{l}{ Cov. estimador } & & \\
\hline 0.104815 & -0.15889 & 0.159858 & 0.001657 \\
-0.15889 & 0.240853 & -0.24234 & -0.00252 \\
0.159858 & -0.24234 & 0.244067 & 0.002596 \\
0.001657 & -0.00252 & 0.002596 & 0.000101
\end{tabular}

\begin{tabular}{rrrr}
\hline $\mathrm{B}$ & & & \\
\hline 1,689 & 1,115 & 0 & 9 \\
1,115 & 743 & 7 & -4 \\
0 & 7 & 7 & -10 \\
9 & -4 & -10 & 29
\end{tabular}

\begin{tabular}{lrrr}
\hline Correl. & & & \\
\hline 1 & -0.99999 & 0.99947 & 0.51017 \\
-0.99999 & 1 & -0.99952 & -0.51105 \\
0.99947 & -0.99952 & 1 & 0.523797 \\
0.51017 & -0.51105 & 0.523797 & 1
\end{tabular}

\begin{tabular}{rrrr}
\hline A & & & \\
\hline 33,074 & 21,466 & -365 & 14 \\
21,466 & 14,027 & -140 & -171 \\
-365 & -140 & 103 & -186 \\
14 & -171 & -186 & 492 \\
& & & \\
\hline Desv. pad. & & & \\
\hline 0.324 & & & \\
0.491 & & & \\
0.494 & & & \\
0.010 & & &
\end{tabular}




\section{Apêndice $\mathbf{H}$}

\section{Aplicação empírica - outras estimativas}

Neste apêndice apresentamos os resultados obtidos para as durações de negócio e preço para a GE, Microsoft e USD/EUR.

\section{H.1 Resultados para a GE}

A figura H.1 ilustra os dados dessazonalizados da GE. Note que o split das ações do dia 12/03/1997 (primeira observação 16686) produziu uma "quebra" no regime das durações de negociação (trade duration) e de preço (price duration), mas que não aparece nas durações de volume (volume duration). É possível também notar o perfil característico de "U" invertido para o efeito diurno para as durações de negociação e volume, mas um perfil um pouco diferente para as durações de preço.

\section{H.1.1 Duração de negócio}



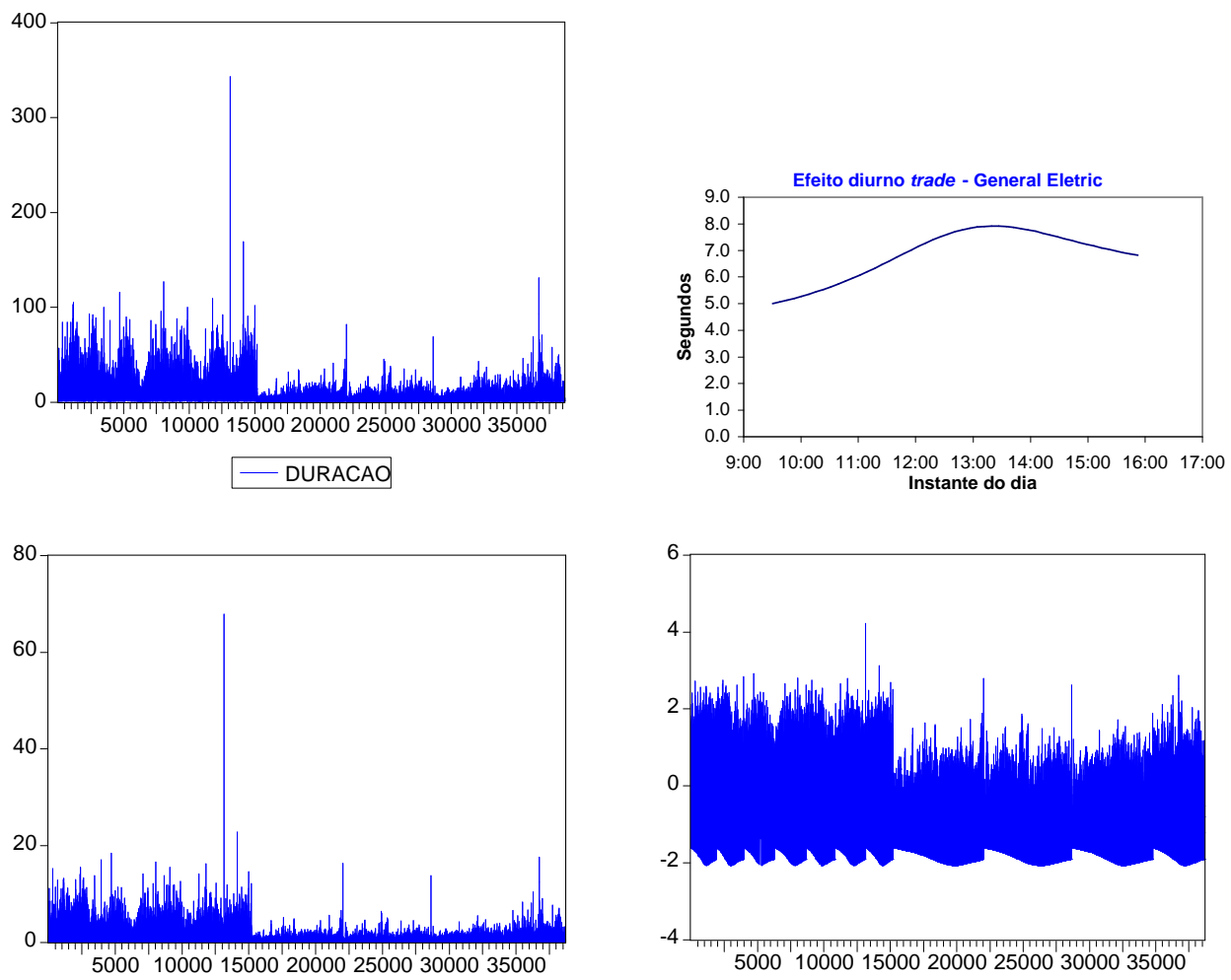

- DURACAO_DESSAZON

- LN_DURACAO_DESSA

Figura H.1: Dados da GE:

Cima: duração de negócio (trade duration) e respectiva componente diurna.

Baixo: duração de negócio dessazonalizada e respectivo logaritmo.

\begin{tabular}{|c|c|c|c|c|c|c|}
\hline \multirow[t]{2}{*}{ Parâmetro } & \multicolumn{3}{|c|}{ Weibull } & \multicolumn{3}{|c|}{ Gamma } \\
\hline & QML & CECF & DECF & QML & CECF & DECF \\
\hline$\delta$ & 0.000 & 0.000 & -0.001 & 0.000 & 0.000 & -0.001 \\
\hline$\phi$ & 0.999 & 0.999 & 0.999 & 0.999 & 0.999 & 0.999 \\
\hline$\sigma_{\eta}$ & 0.022 & 0.028 & 0.031 & 0.022 & 0.028 & 0.030 \\
\hline$\lambda$ & 1.461 & 1.474 & 1.381 & 1.739 & 1.825 & 1.732 \\
\hline Tempo (s) & 17.2 & 13.4 & 51.4 & 11.9 & 13.5 & 7.4 \\
\hline
\end{tabular}

Tabela H.1: Estimação dos parâmetros do modelo SCD para a duração de negócio (trade duration) da GE, considerando distribuições Weibull e gama para os erros. 

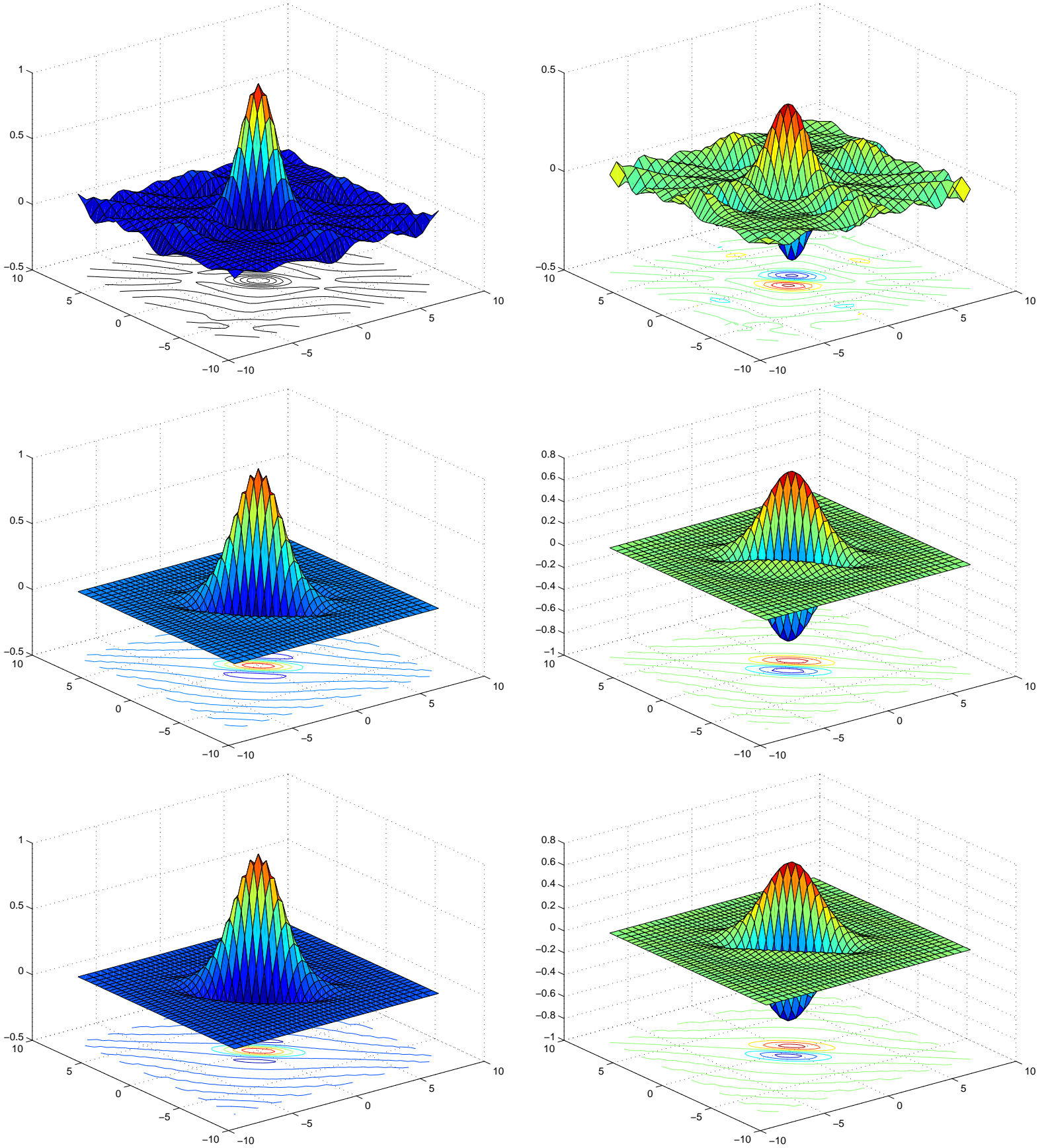

Figura H.2: Partes real (esquerda) e imaginária (direita) da função característica empírica (cima) e das funções características com distribuição gama para a duração de negócio (trade duration) da GE, calculadas em $\theta_{Q M L}$ (meio) e $\theta_{C E C F}$ (baixo), para a malha de 39 nós da quadratura de Gauss-Hermite. 

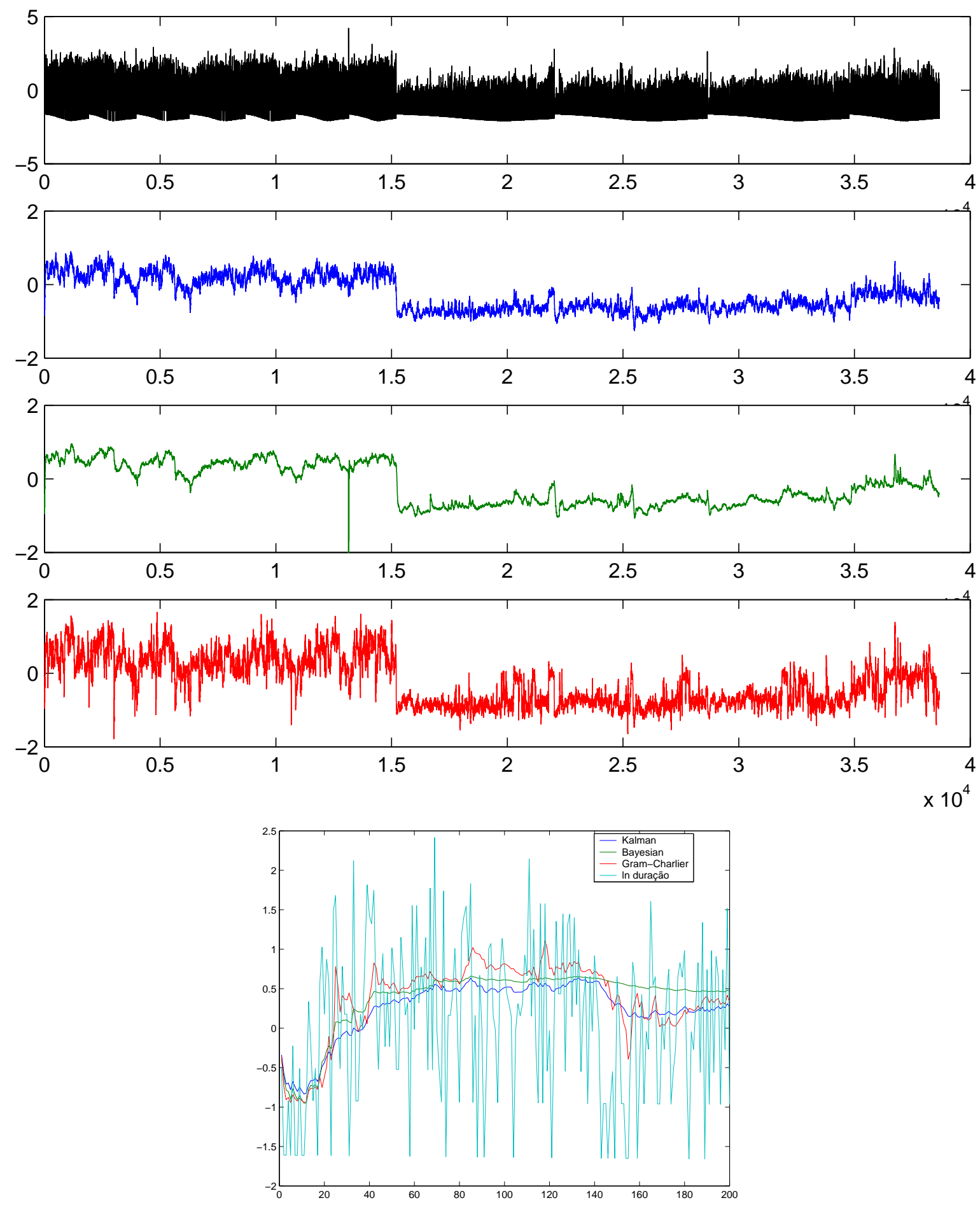

Figura H.3: Estimativa da variável latente para a duração de negócio (trade duration) da GE.

Cima: de cima para baixo temos os seguinte filtros calculados em $\theta_{C E C F}$ : (1) Log-durações; (2) filtro de Kalman; (3) filtro Bayesiano; (4) filtro Gram-Charlier.

Baixo: primeiras 200 observações dos filtros. 

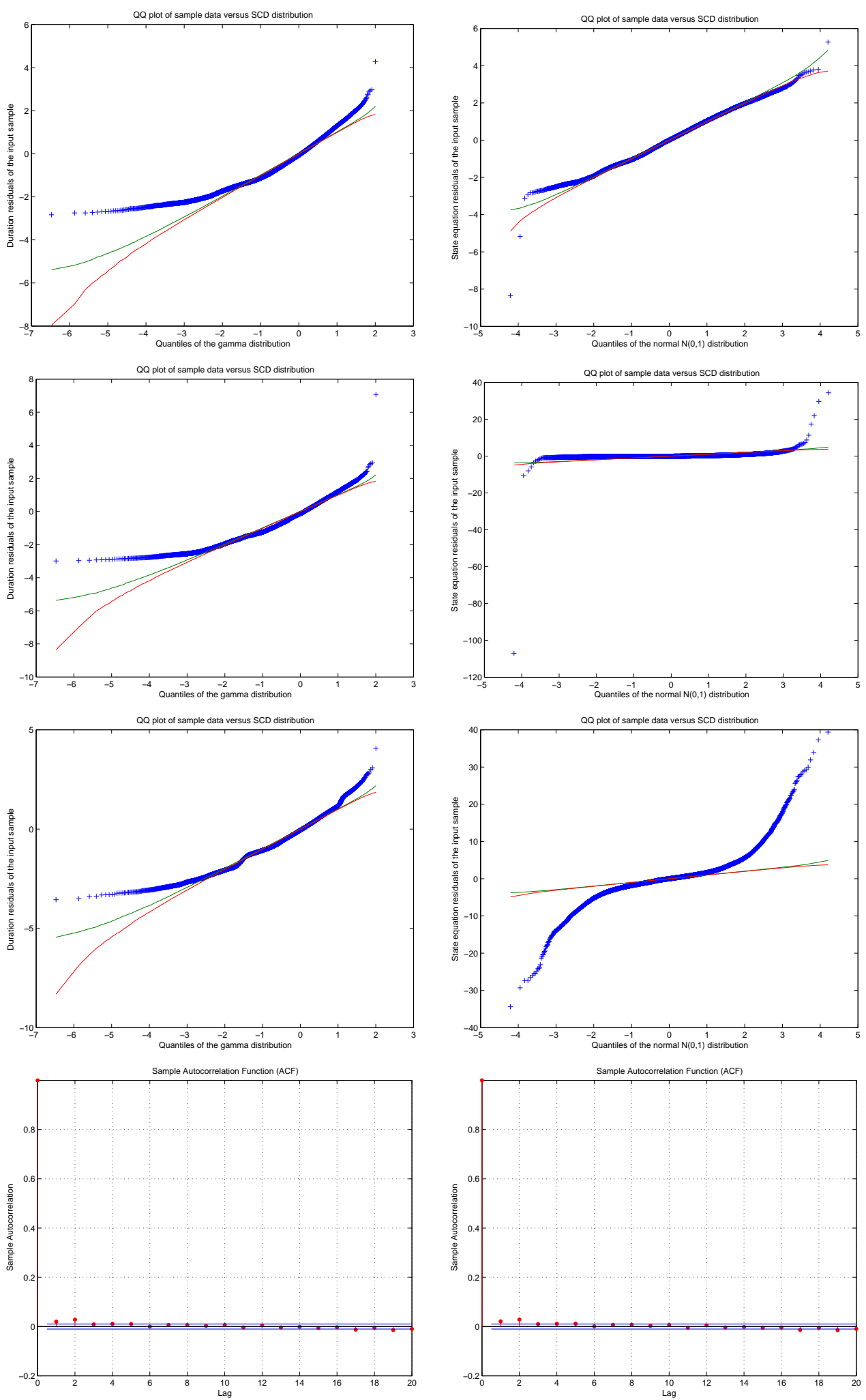

Figura H.4: De cima para baixo temos os gráficos de quantis dos resíduos obtidos com os filtros calculados em $\theta_{C E C F}$ com distribuição gama para a duração de negócio da GE: (1) filtro de Kalman; (2) filtro Bayesiano; (3) filtro Gram-Charlier; (4) função de autocorrelação dos resíduos do filtro de Kalman.

À esquerda vemos os resíduos da equação de observação e à direita os resíduos da equação de estado. As linhas representam os limites do intervalo de $95 \%$ de confiança, obtidos através de simulação. 

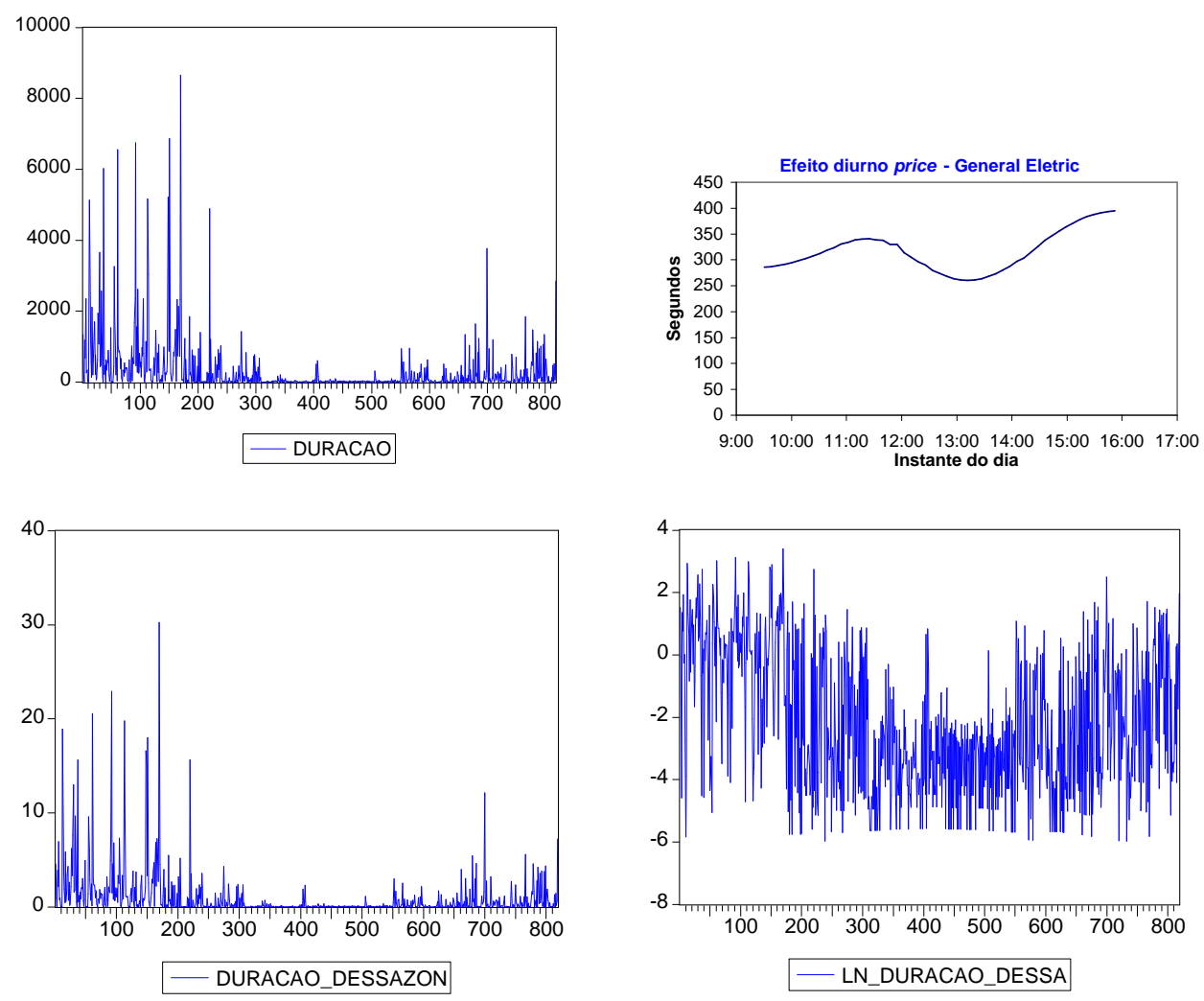

Figura H.5: Dados da GE:

Cima: duração de preço (price duration) e respectiva componente diurna. Baixo: duração de preço dessazonalizada e respectivo logaritmo.

\begin{tabular}{|c|c|c|c|c|c|c|}
\hline \multirow[t]{2}{*}{ Parâmetro } & \multicolumn{3}{|c|}{ Weibull } & \multicolumn{3}{|c|}{ Gamma } \\
\hline & QML & CECF & DECF & QML & CECF & DECF \\
\hline$\delta$ & -0.009 & -0.015 & -0.015 & -0.010 & -0.017 & -0.017 \\
\hline$\phi$ & 0.989 & 0.989 & 0.989 & 0.989 & 0.989 & 0.989 \\
\hline$\sigma_{\eta}$ & 0.194 & 0.247 & 0.271 & 0.194 & 0.265 & 0.293 \\
\hline$\lambda$ & 0.700 & 0.613 & 0.596 & 0.630 & 0.534 & 0.501 \\
\hline Tempo (s) & 1.0 & 10.7 & 22.7 & 0.3 & 10.3 & 13.5 \\
\hline
\end{tabular}

Tabela H.2: Estimação dos parâmetros do modelo SCD para a duração de preço (price duration) da GE, considerando distribuições Weibull e gama para os erros.

\section{H.1.2 Duração de preço}



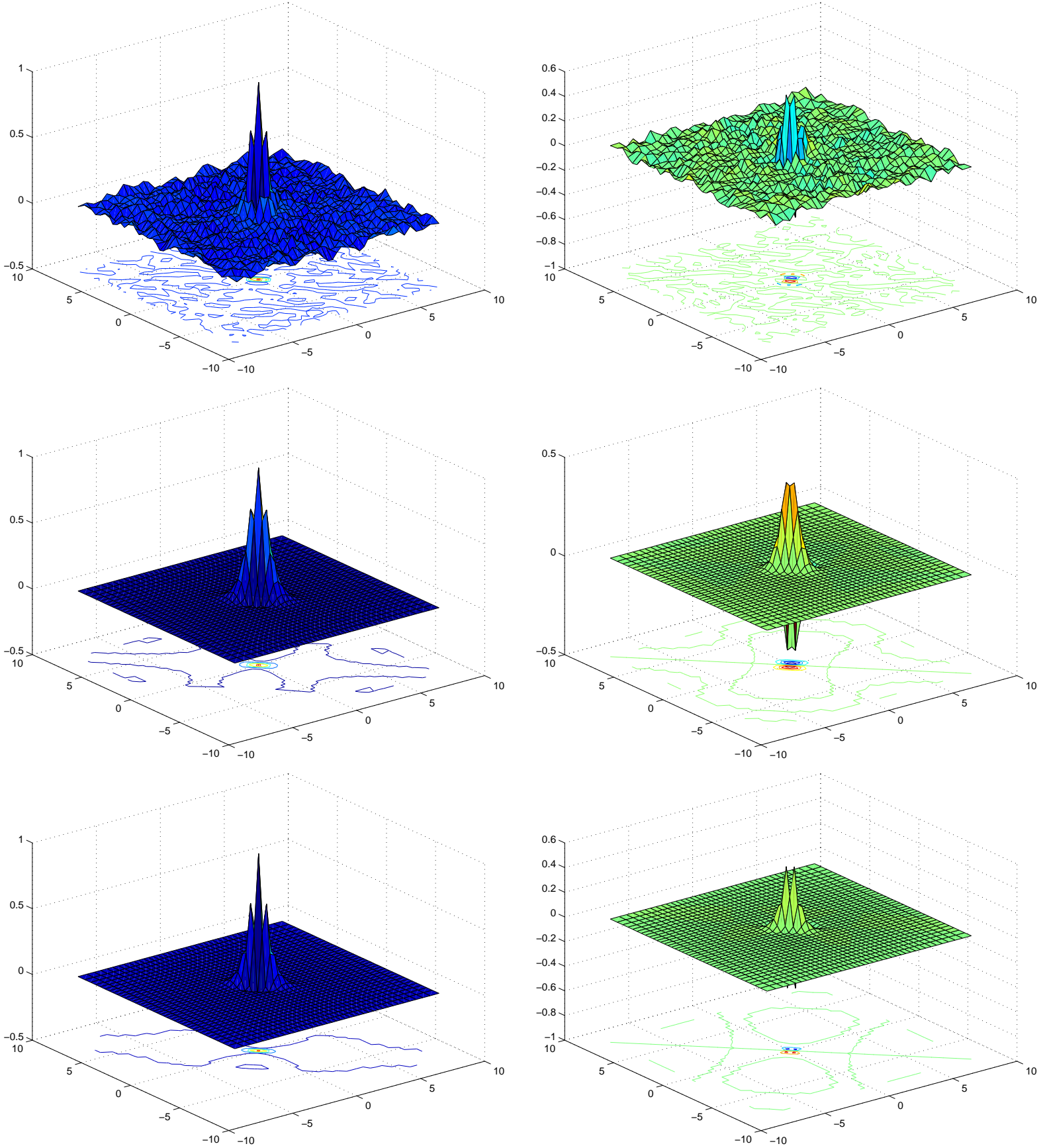

Figura H.6: Partes real (esquerda) e imaginária (direita) da função característica empírica (cima) e das funções características com distribuição gama para a duração de preço (price duration) da GE, calculadas em $\theta_{Q M L}$ (meio) e $\theta_{C E C F}$ (baixo), para a malha de 39 nós da quadratura de Gauss-Hermite. 

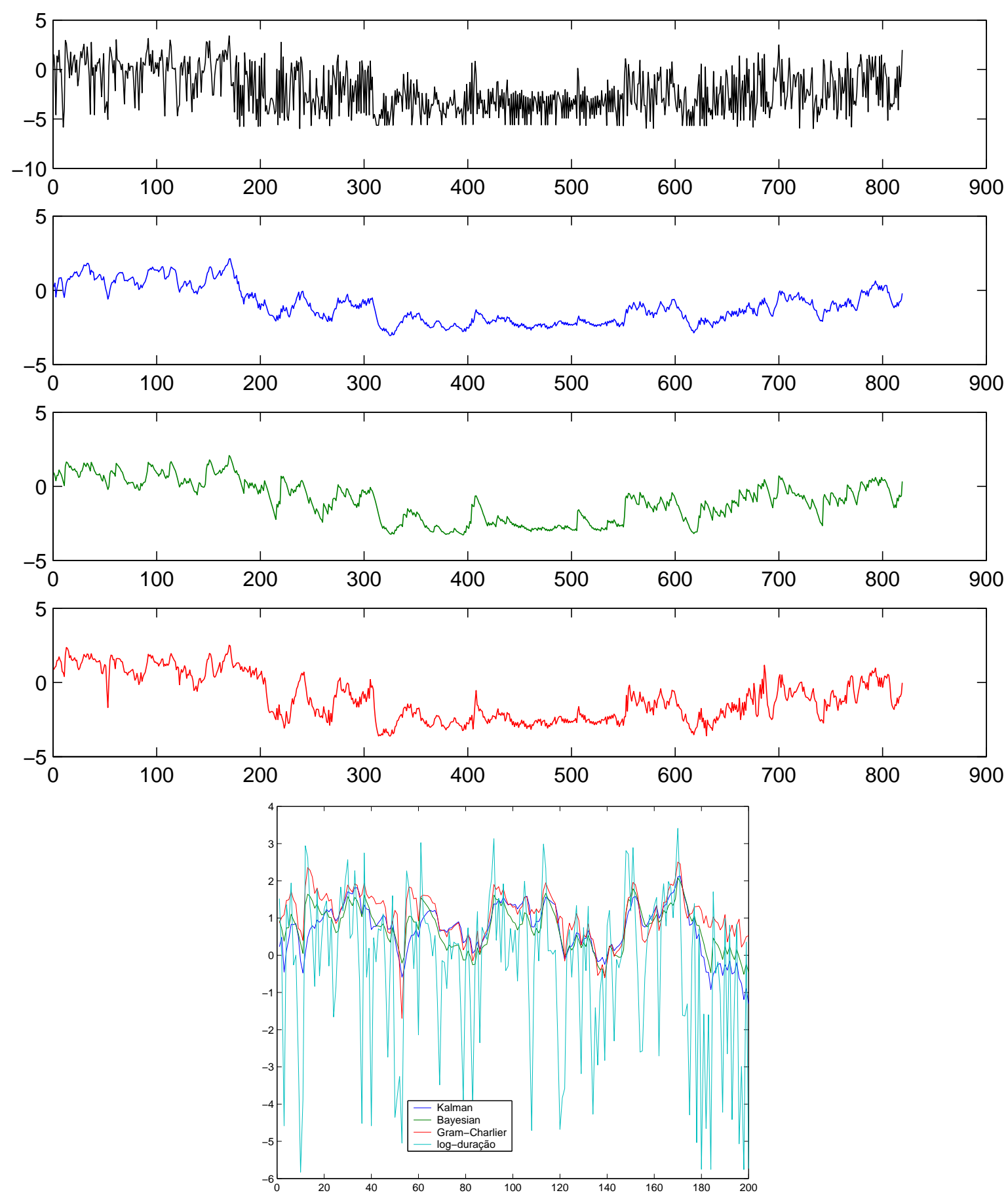

Figura H.7: Estimativa da variável latente para a duração de preço (price duration) da GE. Cima: de cima para baixo temos os seguinte filtros calculados em $\theta_{C E C F}$ : (1) Log-durações; (2) filtro de Kalman; (3) filtro Bayesiano; (4) filtro Gram-Charlier.

Baixo: primeiras 200 observações dos filtros. 

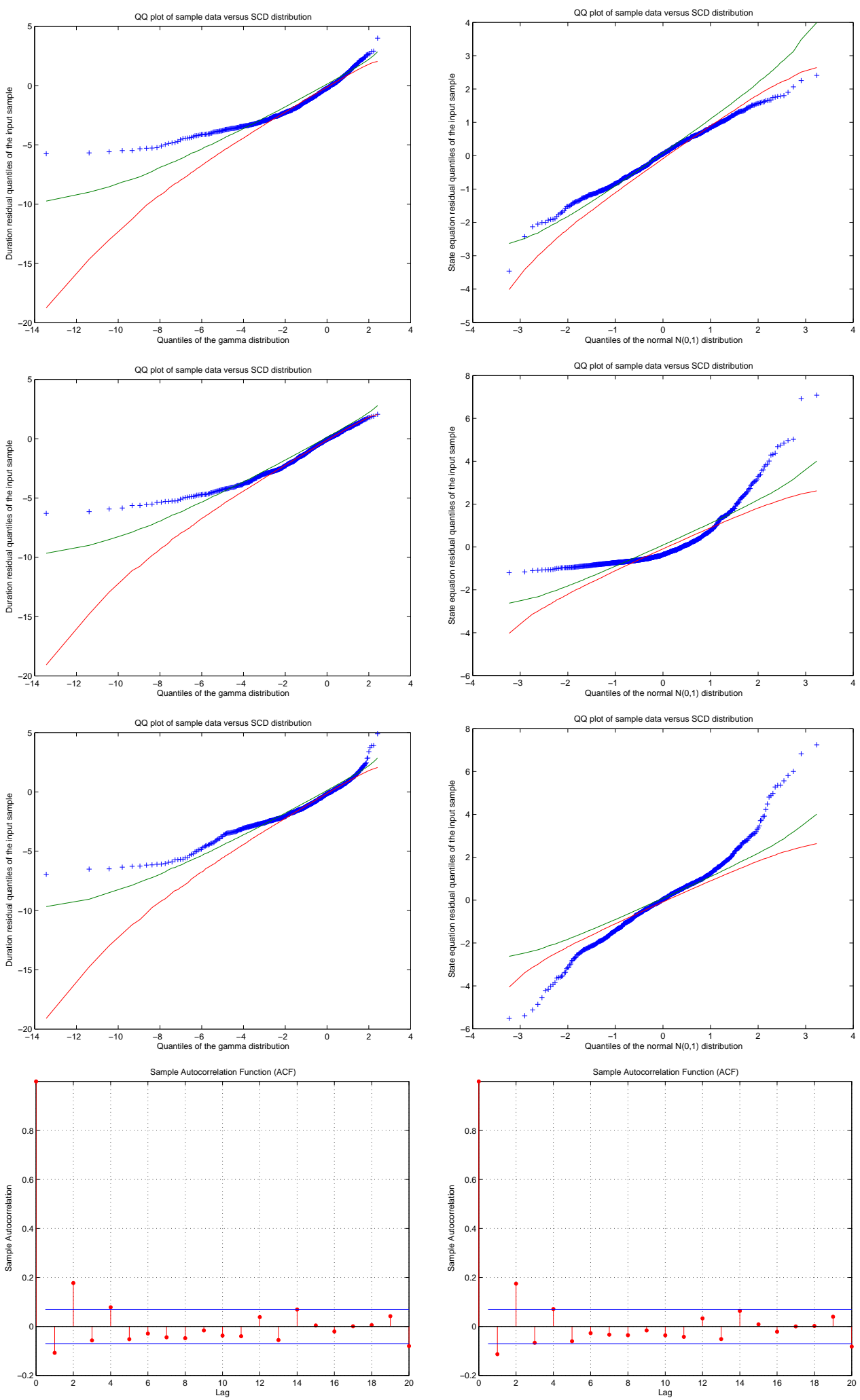

Figura H.8: De cima para baixo temos os gráficos de quantis dos resíduos obtidos com os filtros calculados em $\theta_{C E C F}$ com distribuição gama para a duração de preço da GE: (1) filtro de Kalman; (2) filtro Bayesiano; (3) filtro Gram-Charlier; (4) função de autocorrelação dos resíduos do filtro de Kalman.

À esquerda vemos os resíduos da equação de observação e à direita os resíduos da equação de estado. As linhas representam os limites do intervalo de $95 \%$ de confiança, obtidos através de simulação. 

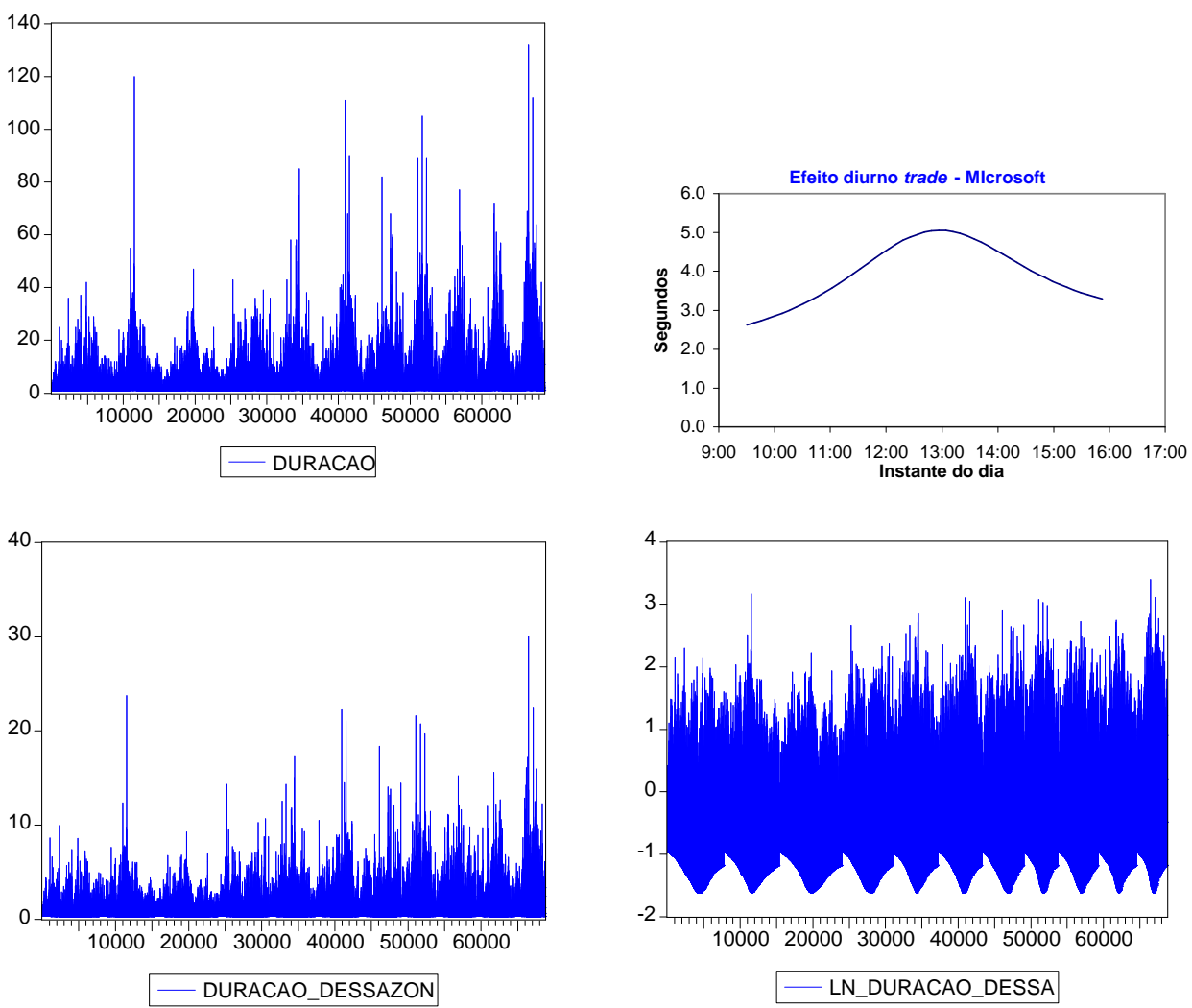

Figura H.9: Dados da Microsoft:

Cima: duração de negócio (trade duration) e respectiva componente diurna.

Baixo: duração de negócio dessazonalizada e respectivo logaritmo.

\begin{tabular}{|c|c|c|c|c|c|c|}
\hline \multirow[t]{2}{*}{ Parâmetro } & \multicolumn{3}{|c|}{ Weibull } & \multicolumn{3}{|c|}{ Gamma } \\
\hline & QML & CECF & DECF & QML & CECF & DECF \\
\hline$\delta$ & -0.004 & -0.008 & -0.012 & -0.004 & -0.007 & -0.011 \\
\hline$\phi$ & 0.977 & 0.977 & 0.977 & 0.977 & 0.978 & 0.977 \\
\hline$\sigma_{\eta}$ & 0.072 & 0.096 & 0.113 & 0.071 & 0.092 & 0.106 \\
\hline$\lambda$ & 1.678 & 1.738 & 1.683 & 2.165 & 2.349 & 2.377 \\
\hline Tempo (s) & 334.1 & 14.1 & 19.6 & 332.6 & 12.2 & 12.2 \\
\hline
\end{tabular}

Tabela H.3: Estimação dos parâmetros do modelo SCD para a duração de negócio (trade duration) da Microsoft, considerando distribuições Weibull e gama para os erros.

\section{H.2 Resultados para a Microsoft}

A figura H.9 ilustra os dados dessazonalizados de Microsoft, que tem comportamento análogo ao da GE.

\section{H.2.1 Duração de negócio}



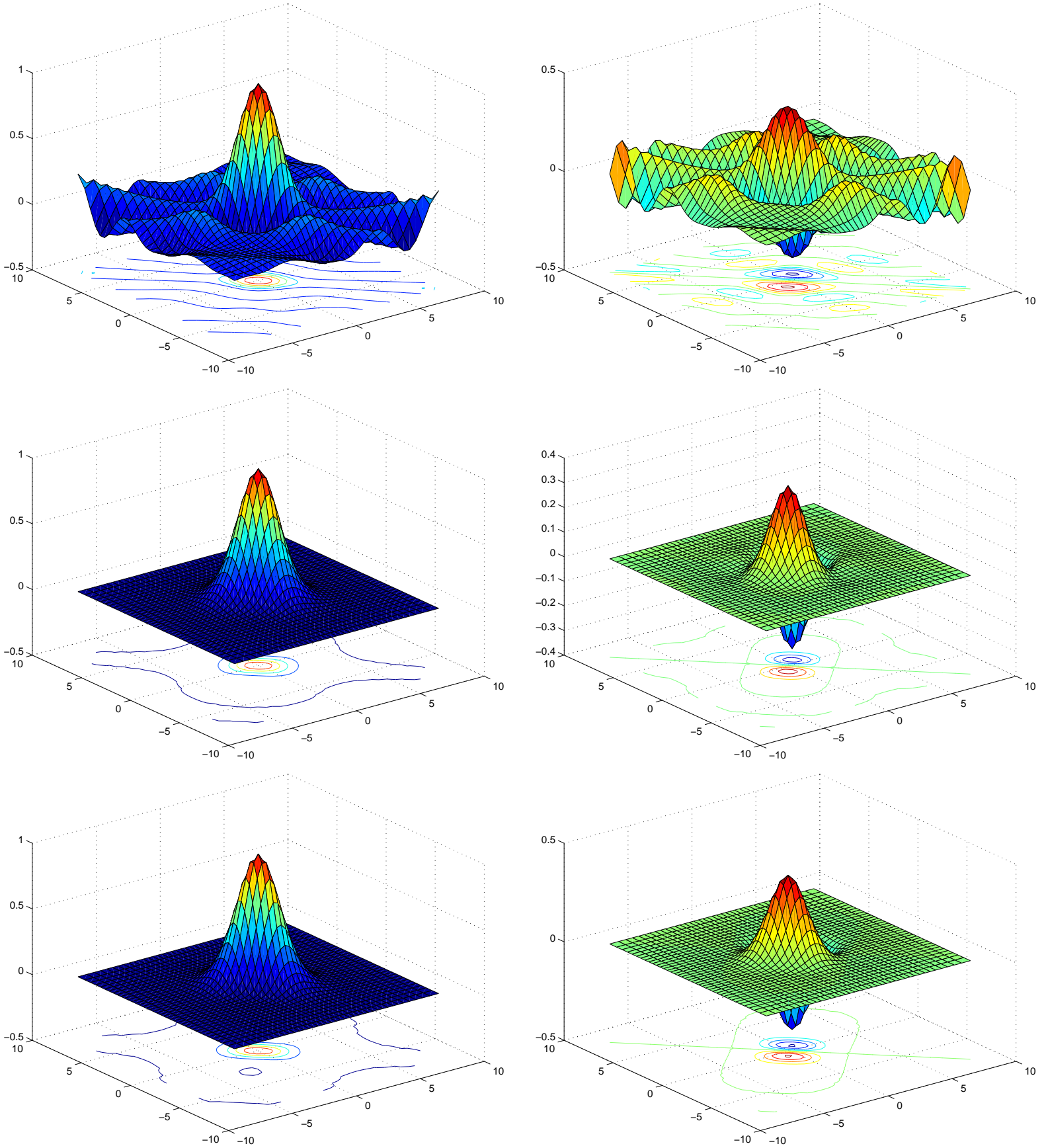

Figura H.10: Partes real (esquerda) e imaginária (direita) da função característica empírica (cima) e das funções características com distribuição gama para a duração de negócio (trade duration) da Microsoft, calculadas em $\theta_{Q M L}$ (meio) e $\theta_{C E C F}$ (baixo), para a malha de 39 nós da quadratura de Gauss-Hermite. 

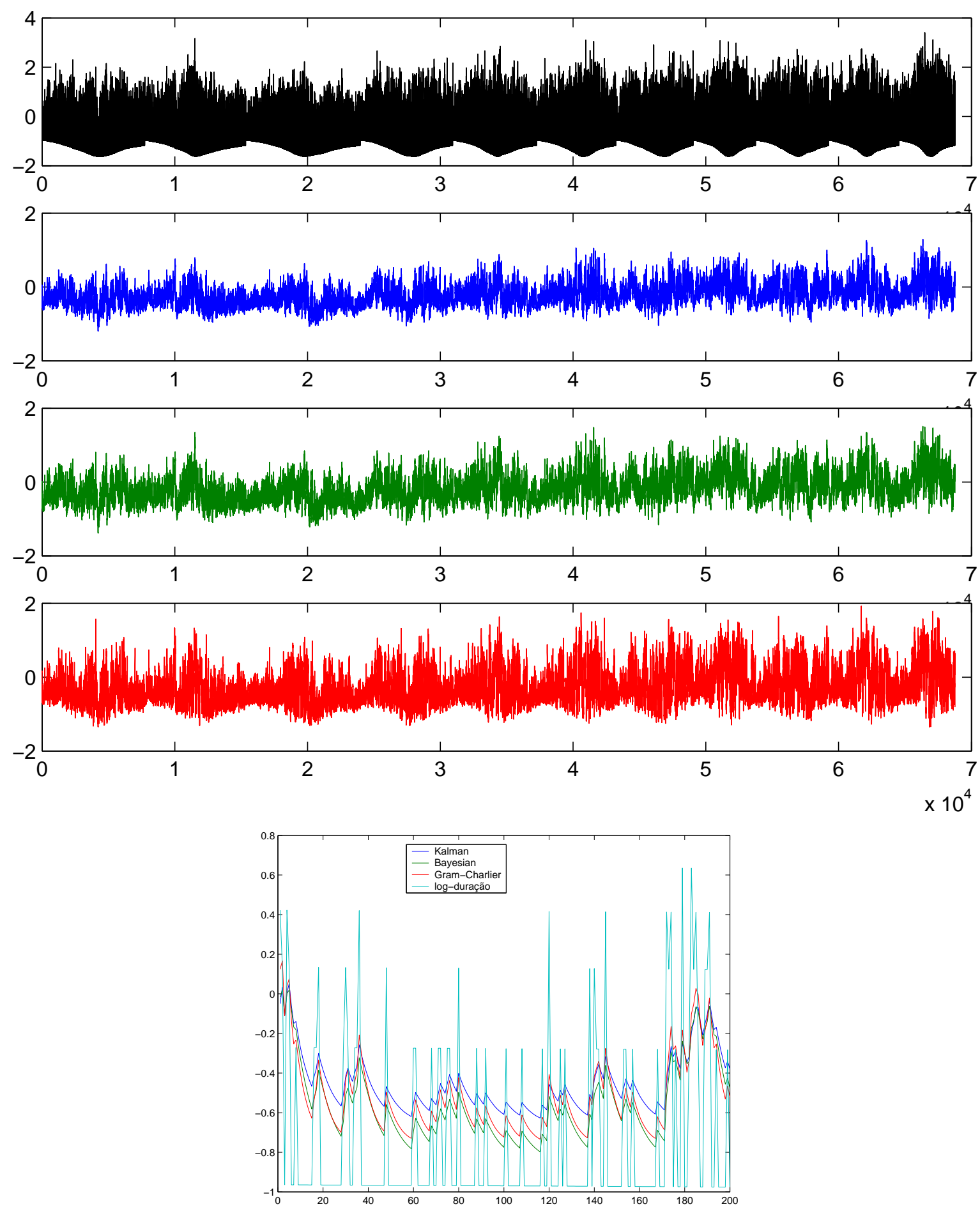

Figura H.11: Estimativa da variável latente para a duração de negócio (trade duration) da Microsoft.

Cima: de cima para baixo temos os seguinte filtros calculados em $\theta_{C E C F}$ : (1) Log-durações; (2) filtro de Kalman; (3) filtro Bayesiano; (4) filtro Gram-Charlier.

Baixo: primeiras 200 observações dos filtros. 

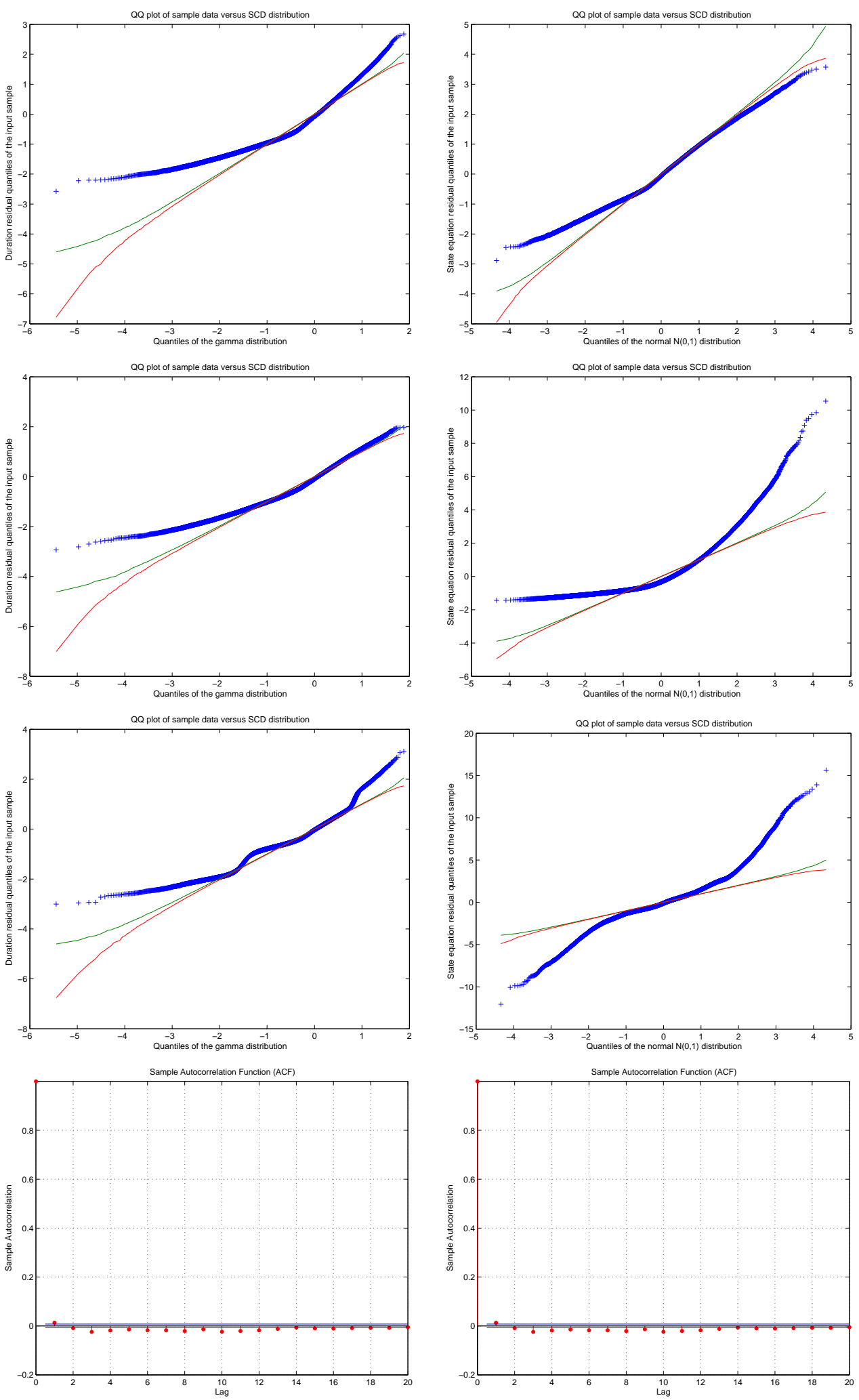

Figura H.12: De cima para baixo temos os gráficos de quantis dos resíduos obtidos com os filtros calculados em $\theta_{C E C F}$ com distribuição gama para a duração de negócio da Microsoft: (1) filtro de Kalman; (2) filtro Bayesiano; (3) filtro Gram-Charlier; (4) função de autocorrelação dos resíduos do filtro de Kalman.

À esquerda vemos os resíduos da equação de observação e à direita os resíduos da equação de estado. As linhas representam os limites do intervalo de $95 \%$ de confiança, obtidos através de simulação. 

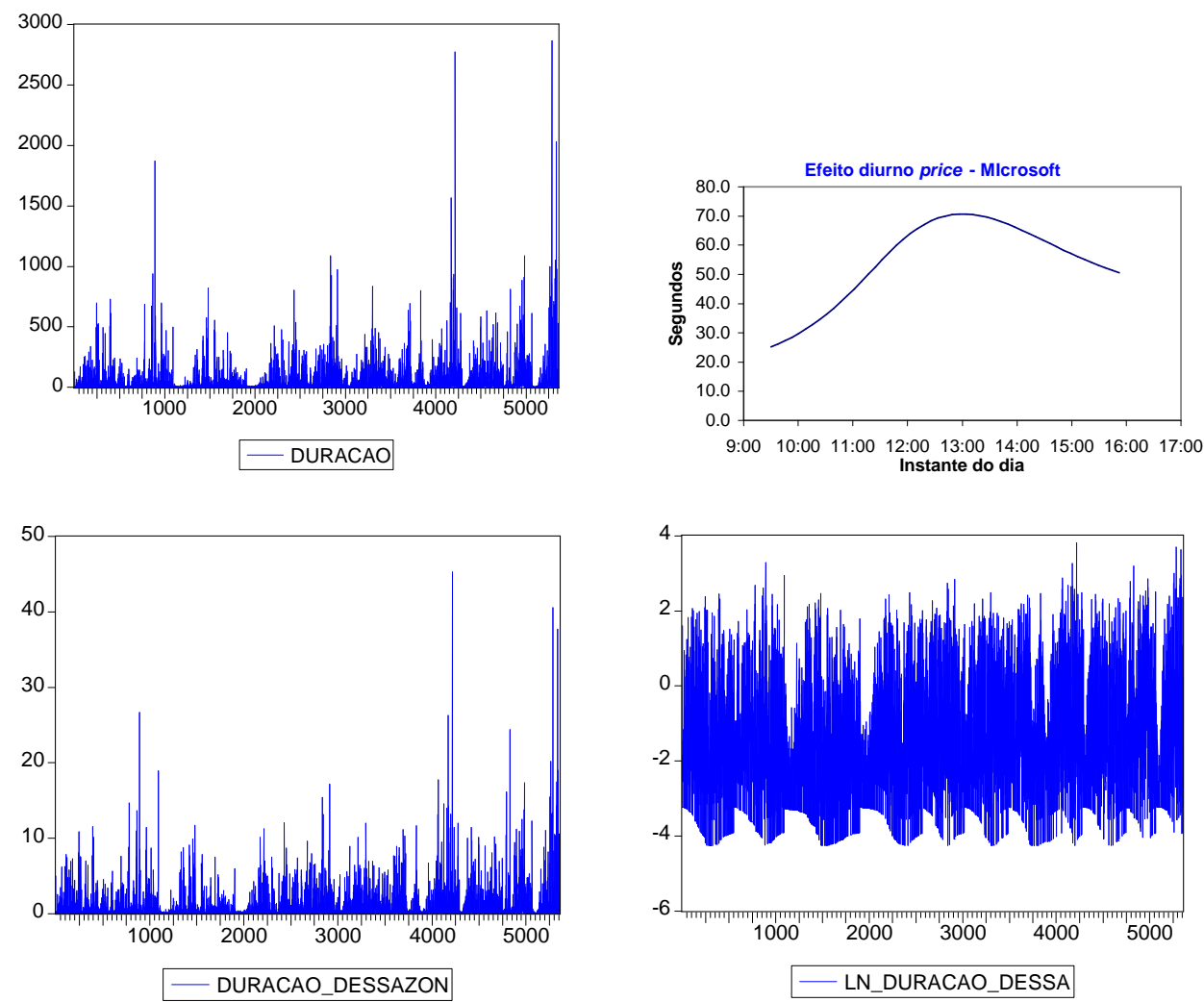

Figura H.13: Dados da Microsoft:

Cima: duração de preço (price duration) e respectiva componente diurna.

Baixo: duração de preço dessazonalizada e respectivo logaritmo.

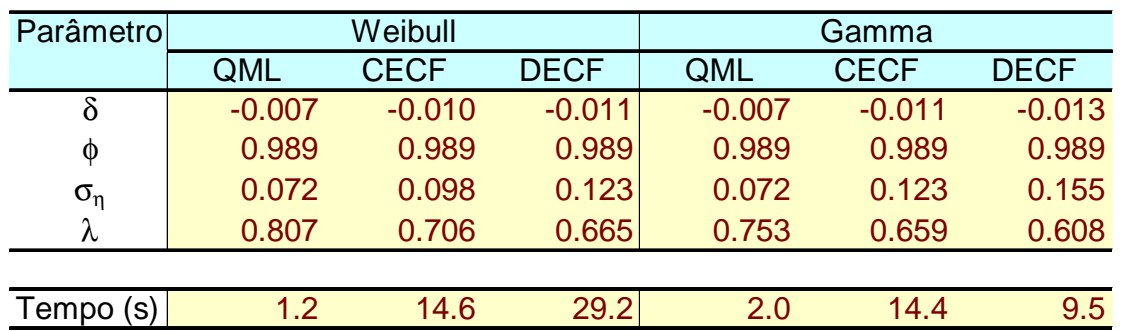

Tabela H.4: Estimação dos parâmetros do modelo SCD para a duração de preço (price duration) da Microsoft, considerando distribuições Weibull e gama para os erros.

\section{H.2.2 Duração de preço}



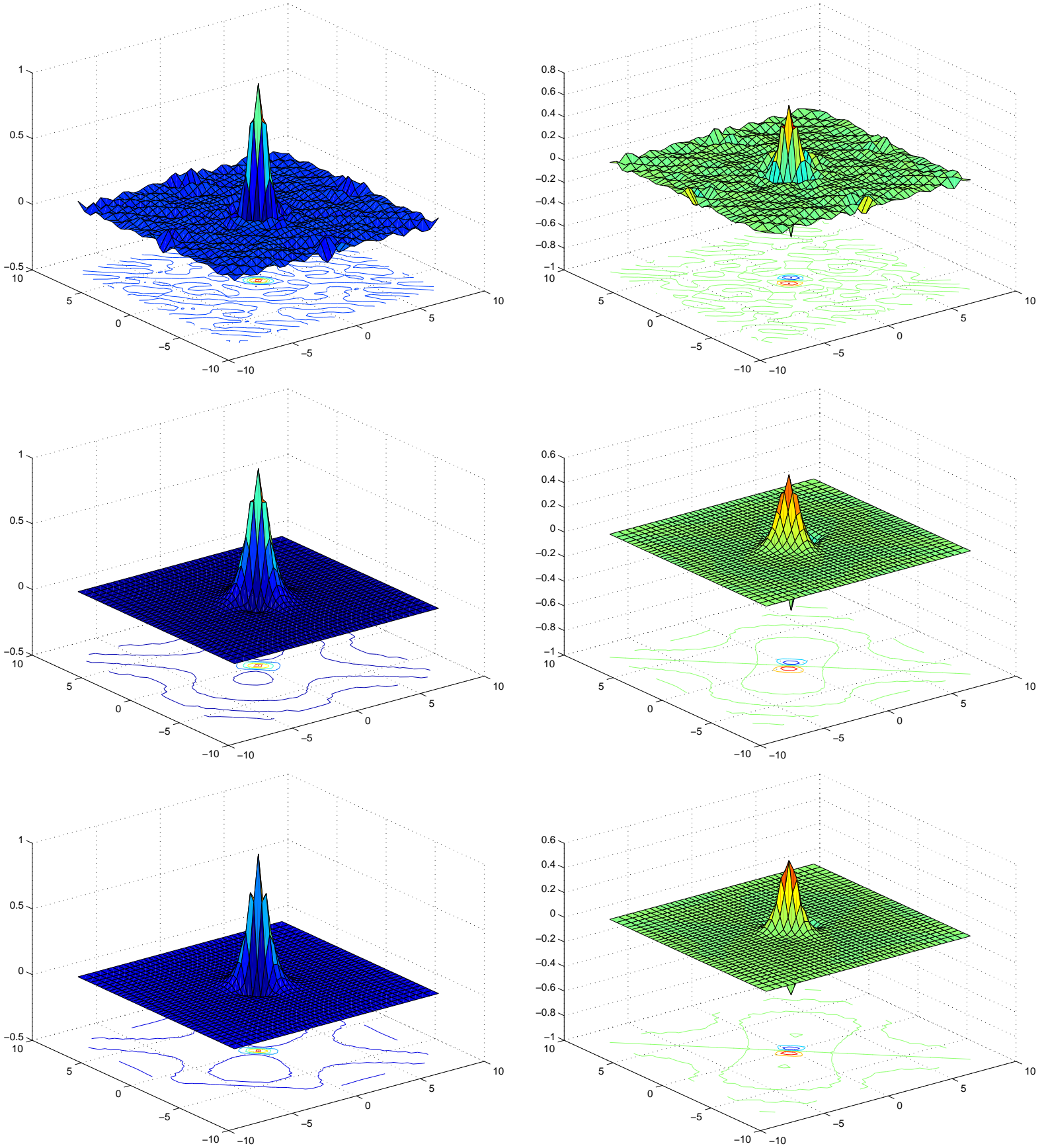

Figura H.14: Partes real (esquerda) e imaginária (direita) da função característica empírica (cima) e das funções características com distribuição gama para a duração de preço (price duration) da Microsoft, calculadas em $\theta_{Q M L}$ (meio) e $\theta_{C E C F}$ (baixo), para a malha de 39 nós da quadratura de Gauss-Hermite. 

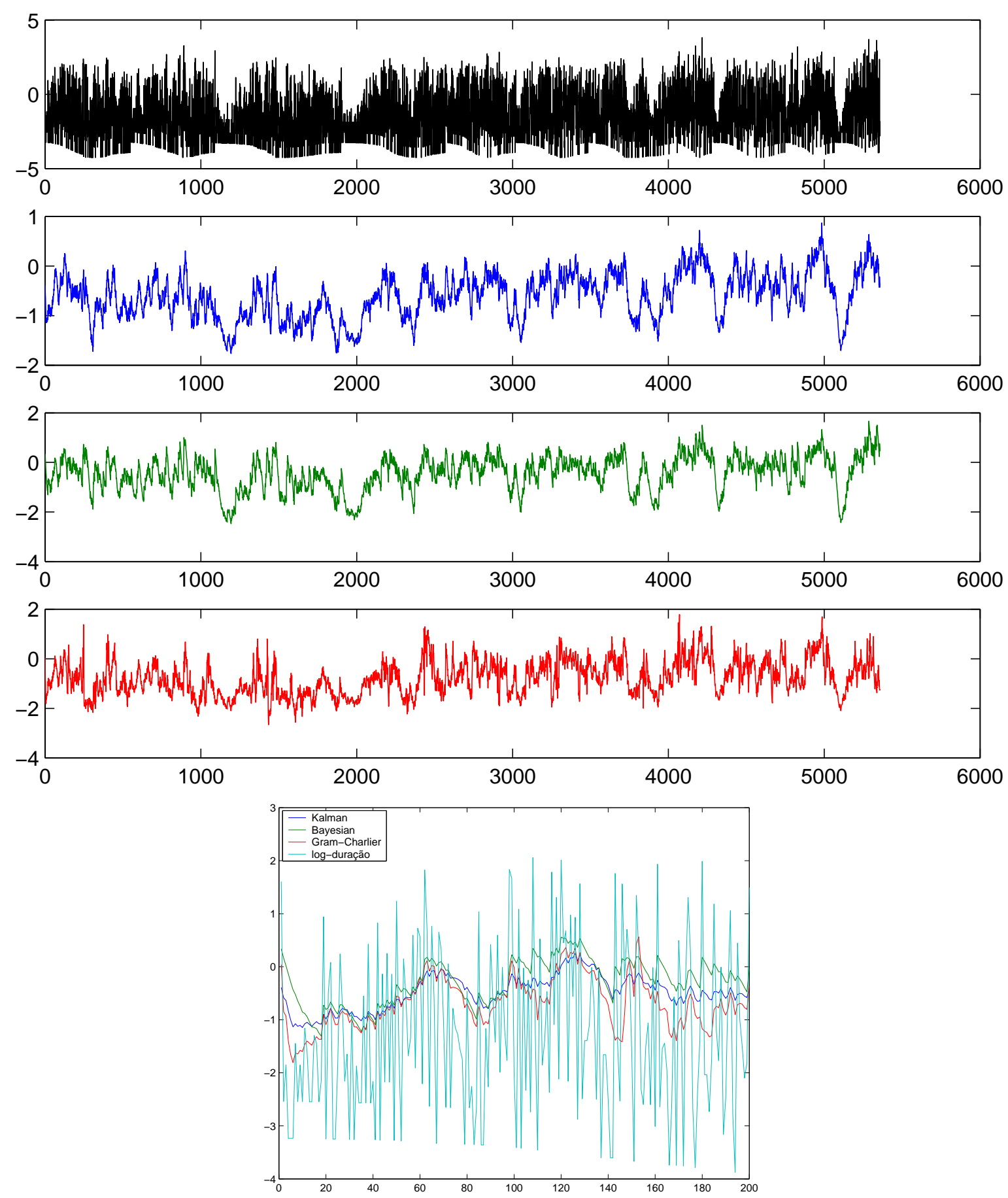

Figura H.15: Estimativa da variável latente para a duração de preço (price duration) da Microsoft.

Cima: de cima para baixo temos os seguinte filtros calculados em $\theta_{C E C F}$ : (1) Log-durações; (2) filtro de Kalman; (3) filtro Bayesiano; (4) filtro Gram-Charlier. Baixo: primeiras 200 observações dos filtros. 

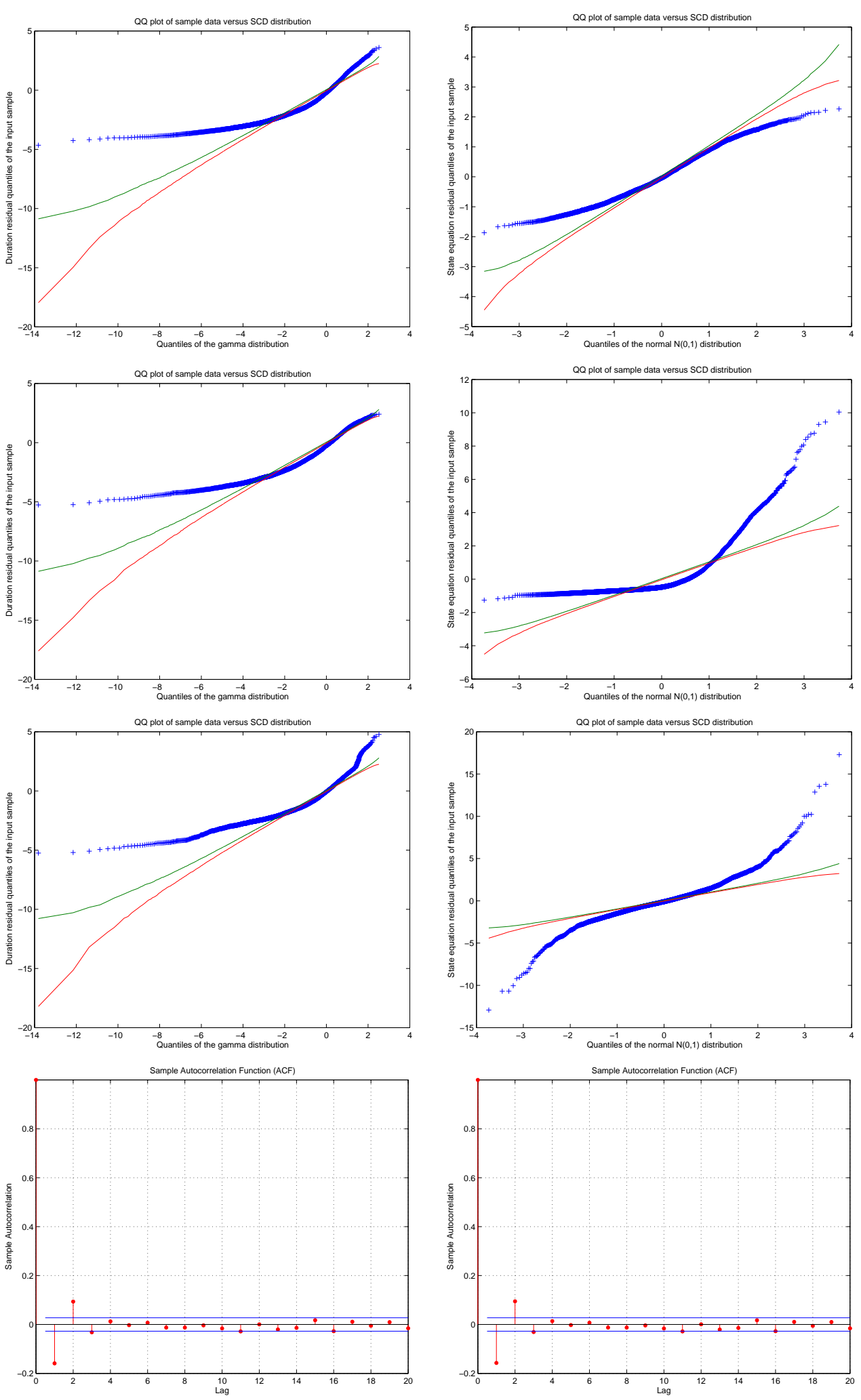

Figura H.16: De cima para baixo temos os gráficos de quantis dos resíduos obtidos com os filtros calculados em $\theta_{C E C F}$ com distribuição gama para a duração de preço da Microsoft: (1) filtro de Kalman; (2) filtro Bayesiano; (3) filtro Gram-Charlier; (4) função de autocorrelação dos resíduos do filtro de Kalman.

À esquerda vemos os resíduos da equação de observação e à direita os resíduos da equação de estado. As linhas representam os limites do intervalo de $95 \%$ de confiança, obtidos através de simulação. 

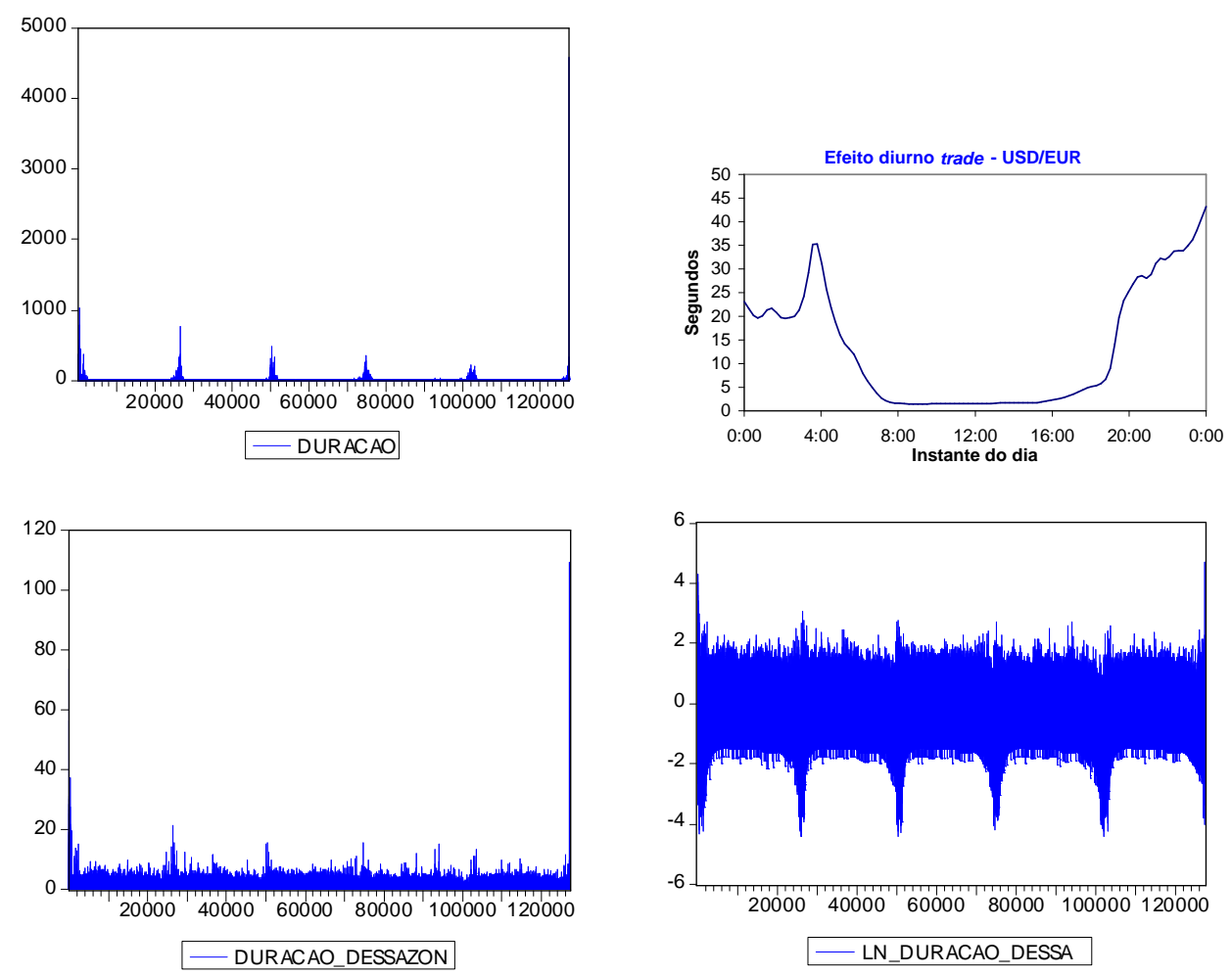

Figura H.17: Dados de USD/EUR:

Cima: duração de negócio (trade duration) e respectiva componente diurna.

Baixo: duração de negócio dessazonalizada e respectivo logaritmo.

\section{H.3 Resultados para USD/EUR}

A figura $[$ H.17 ilustra os dados dessazonalizados de USD/EUR. Note que o perfil do efeito diurno é bastante diferente das ações da NYSE, com menores durações (maior atividade do mercado) entre 8:00 e 16:30, de maneira praticamente uniforme neste intervalo e aumentando a duração fora do intervalo. Além disso, mesmo utilizando uma largura de banda pequena para capturar maiores detalhes, não conseguimos eliminar completamente o efeito diurno nas durações de negociação. Por isso, propomos um outro método de dessazonalização em uma seção a seguir.

\section{H.3.1 Duração de negócio}




\begin{tabular}{|c|c|c|c|c|c|c|}
\hline \multirow[t]{2}{*}{ Parâmetro } & \multicolumn{3}{|c|}{ Weibull } & \multicolumn{3}{|c|}{ Gamma } \\
\hline & QML & CECF & DECF & QML & CECF & DECF \\
\hline$\delta$ & 0.000 & 0.000 & -0.001 & 0.000 & 0.000 & 0.000 \\
\hline$\phi$ & 0.998 & 0.998 & 0.998 & 0.998 & 0.998 & 0.998 \\
\hline$\sigma_{\eta}$ & 0.010 & 0.016 & 0.019 & 0.010 & 0.010 & 0.017 \\
\hline$\lambda$ & 1.799 & 1.888 & 1.923 & 2.428 & 2.541 & 2.957 \\
\hline Tempo (s) & 495.5 & 16.3 & 16.6 & 495.9 & 8.2 & $\overline{40.4}$ \\
\hline
\end{tabular}

Tabela H.5: Estimação dos parâmetros do modelo SCD para a duração de negócio (trade duration) de USD/EUR, considerando distribuições Weibull e gama para os erros. 

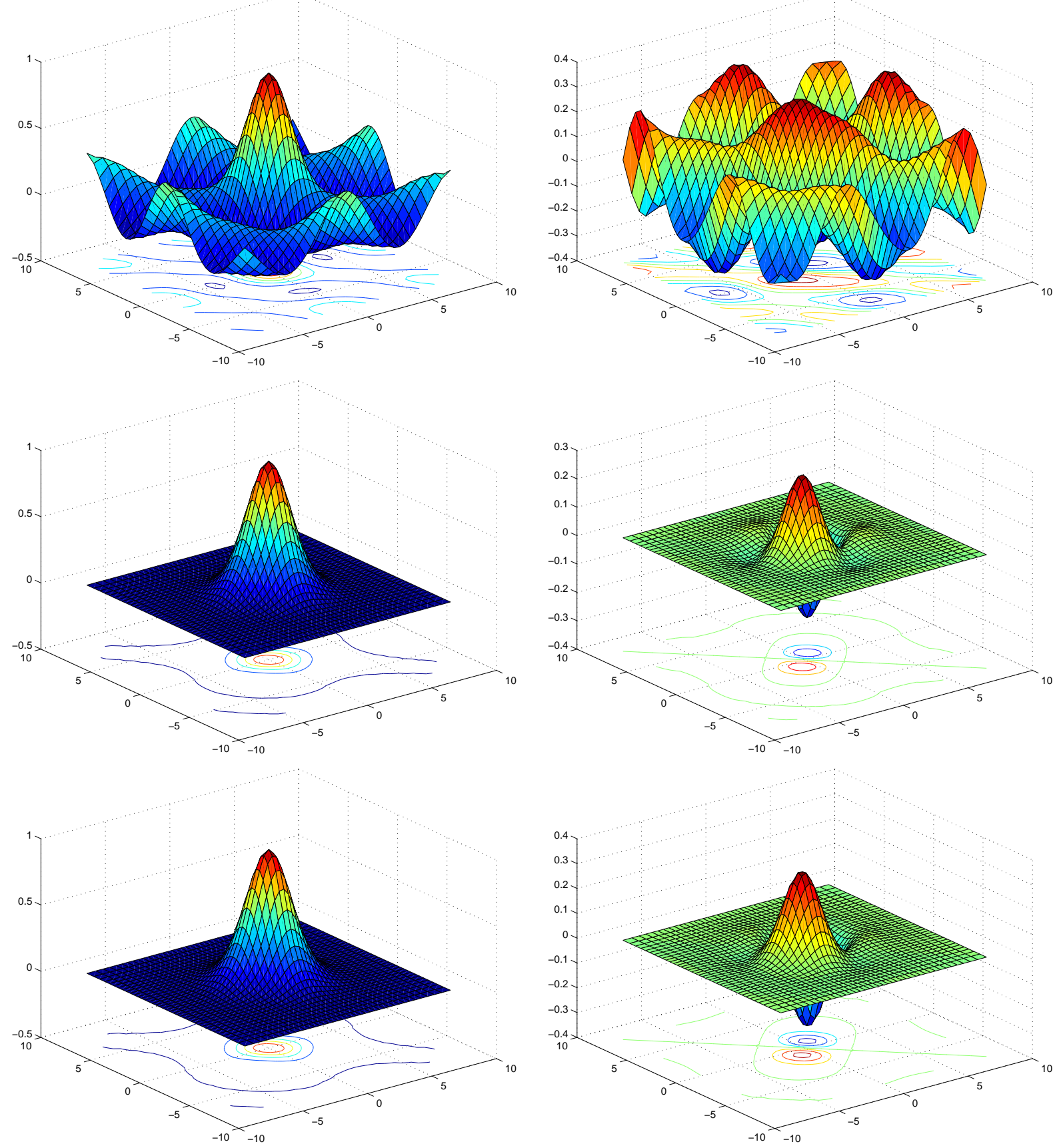

Figura H.18: Partes real (esquerda) e imaginária (direita) da função característica empírica (cima) e das funções características com distribuição gama para a duração de negócio (trade duration) de USD/EUR, calculadas em $\theta_{Q M L}$ (meio) e $\theta_{C E C F}$ (baixo), para a malha de 39 nós da quadratura de Gauss-Hermite. 

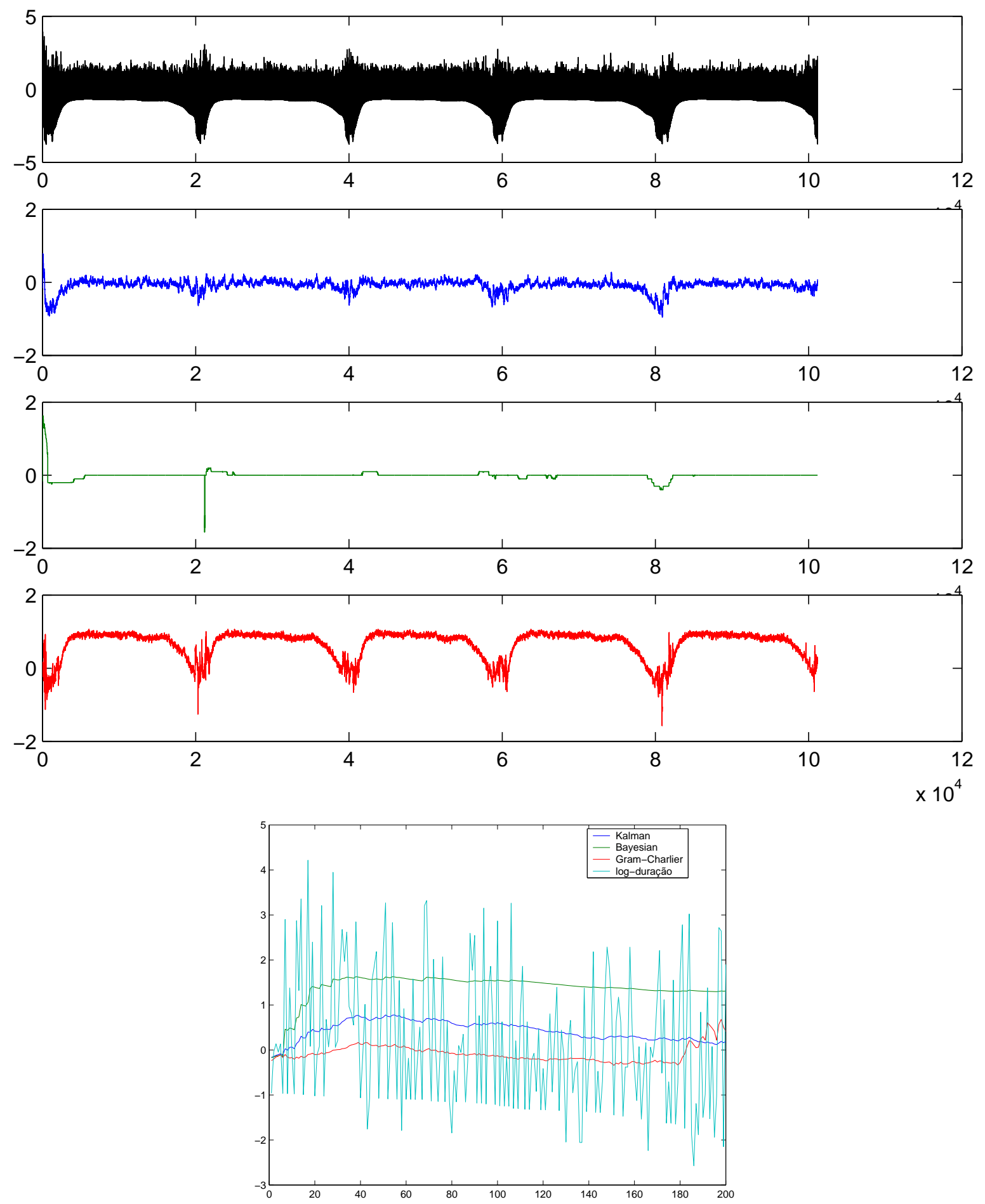

Figura H.19: Estimativa da variável latente para a duração de negócio (trade duration) de USD/EUR.

Cima: de cima para baixo temos os seguinte filtros calculados em $\theta_{C E C F}$ : (1) Log-durações; (2) filtro de Kalman; (3) filtro Bayesiano; (4) filtro Gram-Charlier.

Baixo: primeiras 200 observações dos filtros. 

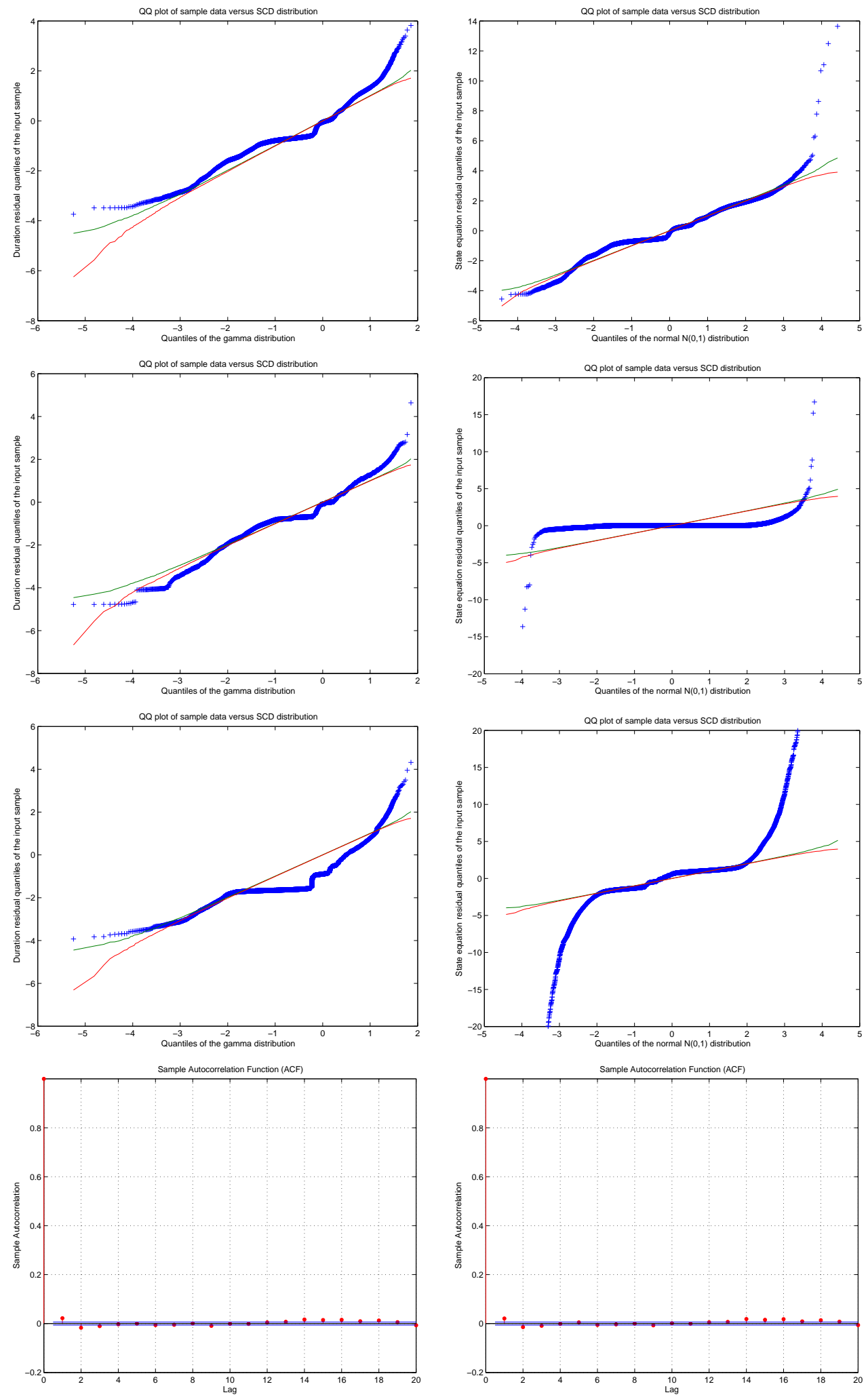

Figura H.20: De cima para baixo temos os gráficos de quantis dos resíduos obtidos com os filtros calculados em $\theta_{C E C F}$ com distribuição gama para a duração de negócio de USD/EUR: (1) filtro de Kalman; (2) filtro Bayesiano; (3) filtro Gram-Charlier; (4) função de autocorrelação dos resíduos do filtro de Kalman.

À esquerda vemos os resíduos da equação de observação e à direita os resíduos da equação de estado. As linhas representam os limites do intervalo de $95 \%$ de confiança, obtidos através de simulação. 

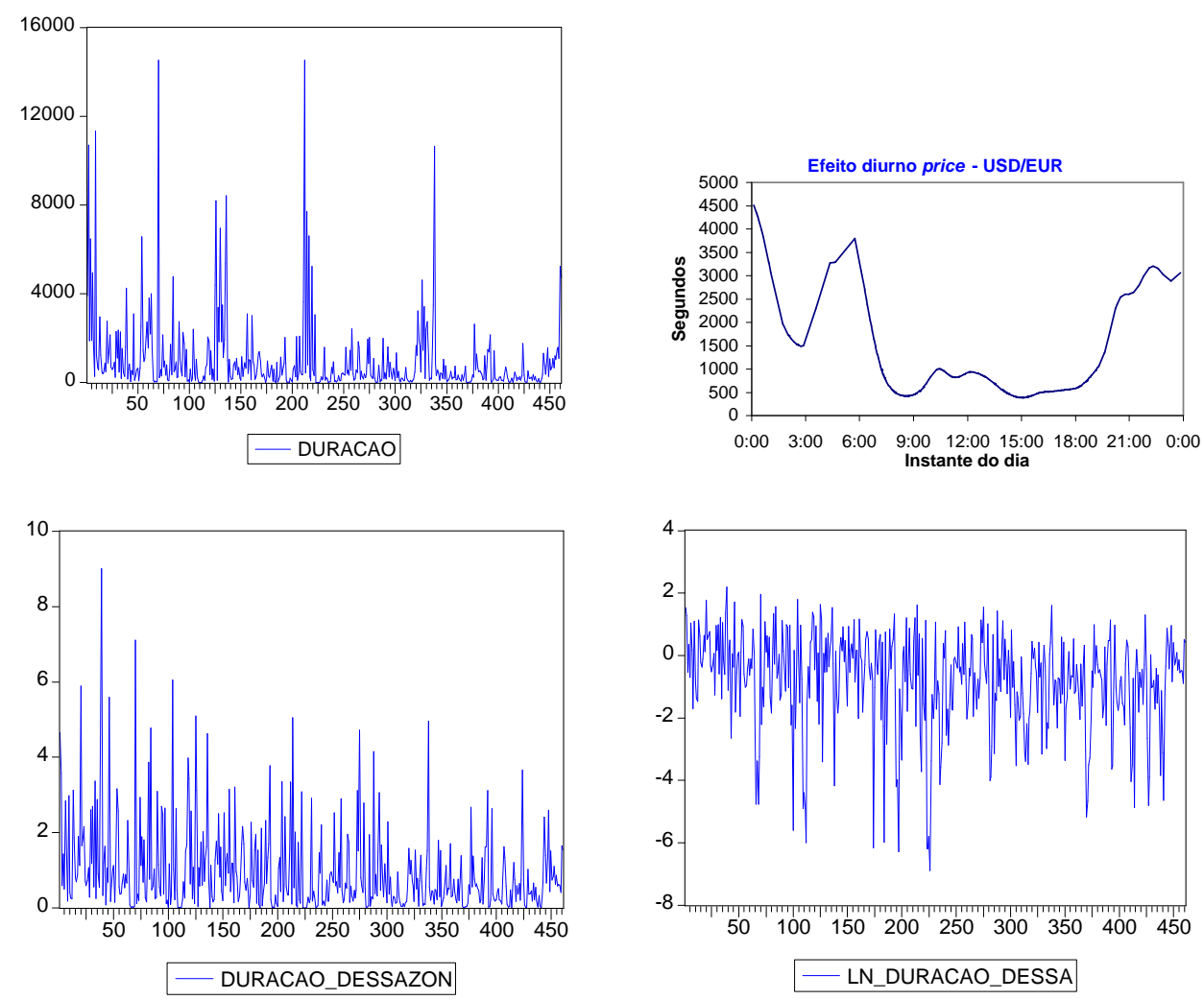

Figura H.21: Dados de USD/EUR:

Cima: duração de preço (price duration) e respectiva componente diurna. Baixo: duração de preço dessazonalizada e respectivo logaritmo.

\begin{tabular}{|c|c|c|c|c|c|c|}
\hline \multirow[t]{2}{*}{ Parâmetro } & \multicolumn{3}{|c|}{ Weibull } & \multicolumn{3}{|c|}{ Gamma } \\
\hline & QML & CECF & DECF & QML & CECF & DECF \\
\hline $\bar{\delta}$ & -0.181 & -0.003 & -0.081 & -0.175 & -0.004 & -0.052 \\
\hline$\phi$ & 0.600 & 0.855 & 0.108 & 0.600 & 0.849 & 0.120 \\
\hline$\sigma_{\eta}$ & 0.997 & 0.228 & 0.739 & 0.997 & 0.233 & 0.685 \\
\hline$\lambda$ & 1.291 & 0.956 & 1.188 & 1.441 & 0.932 & 1.225 \\
\hline Tempo (s) & 0.1 & 47.3 & 34.3 & 0.2 & 40.7 & 21.5 \\
\hline
\end{tabular}

Tabela H.6: Estimação dos parâmetros do modelo SCD para a duração de preço (price duration) de USD/EUR, considerando distribuições Weibull e gama para os erros.

\section{H.3.2 Duração de preço}



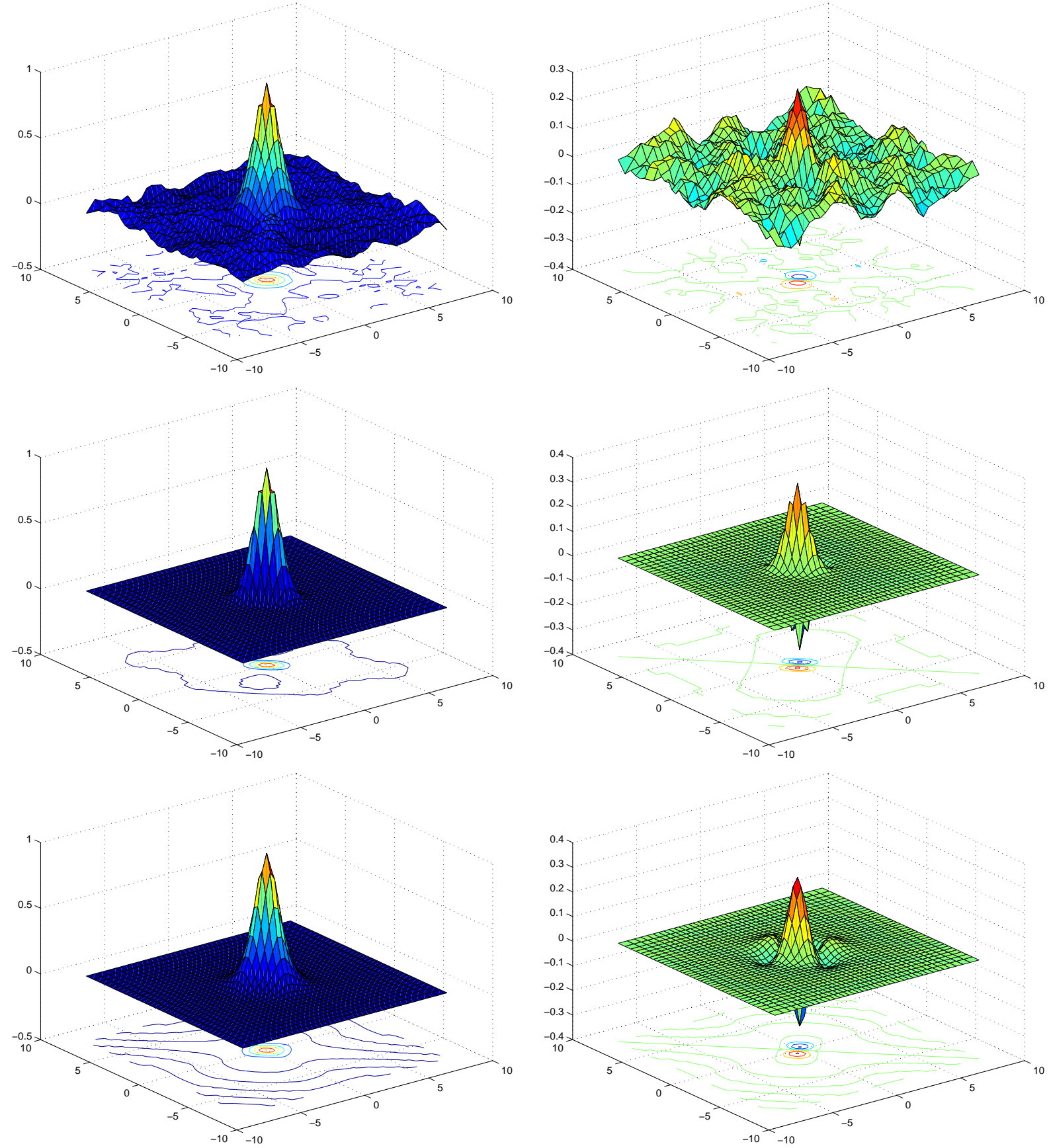

Figura H.22: Partes real (esquerda) e imaginária (direita) da função característica empírica (cima) e das funções características com distribuição gama para a duração de preço (price duration) de USD/EUR, calculadas em $\theta_{Q M L}$ (meio) e $\theta_{C E C F}$ (baixo), para a malha de 39 nós da quadratura de Gauss-Hermite. 

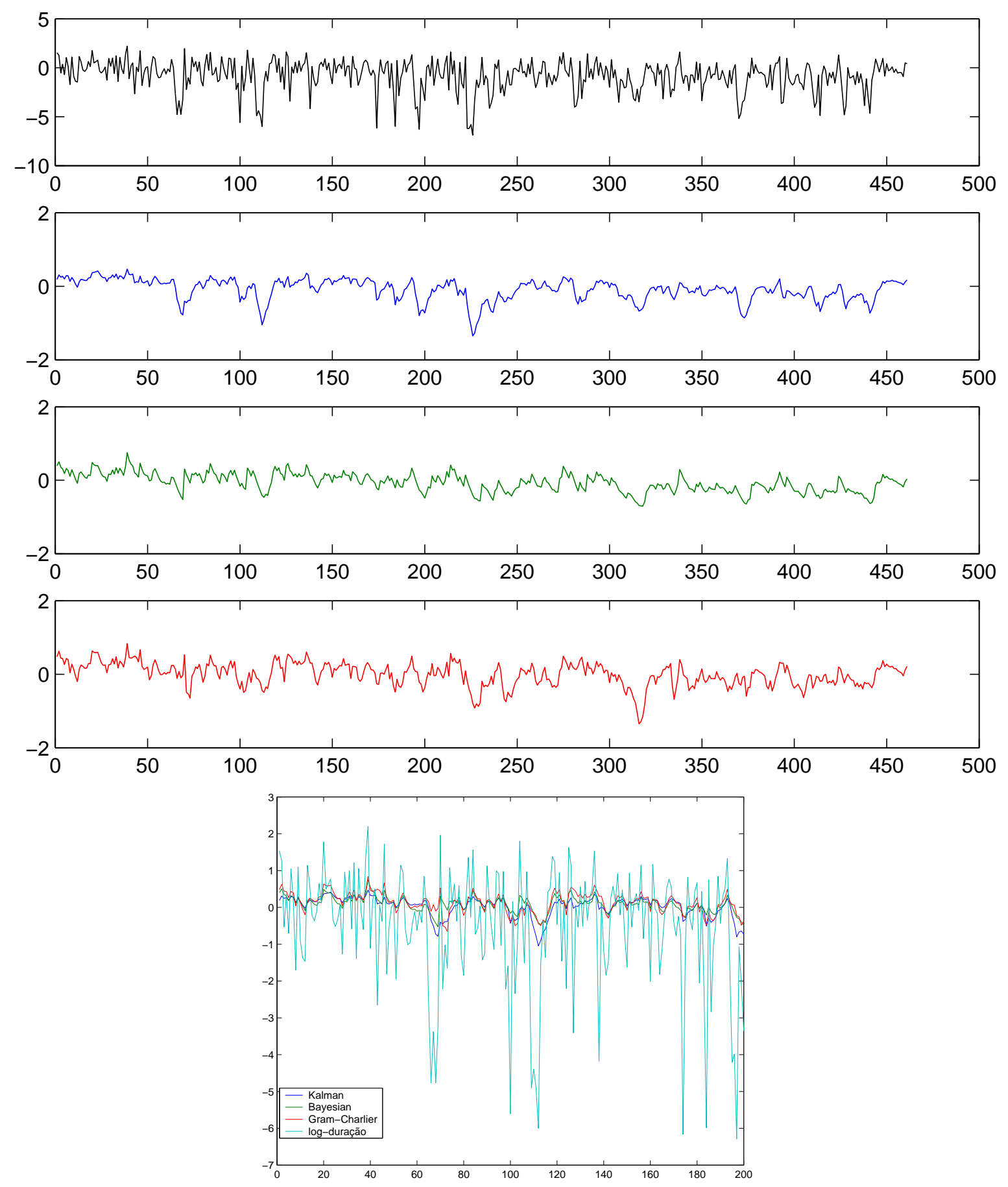

Figura H.23: Estimativa da variável latente para a duração de preço (price duration) de USD/EUR.

Cima: de cima para baixo temos os seguinte filtros calculados em $\theta_{C E C F}$ : (1) Log-durações; (2) filtro de Kalman; (3) filtro Bayesiano; (4) filtro Gram-Charlier.

Baixo: primeiras 200 observações dos filtros. 

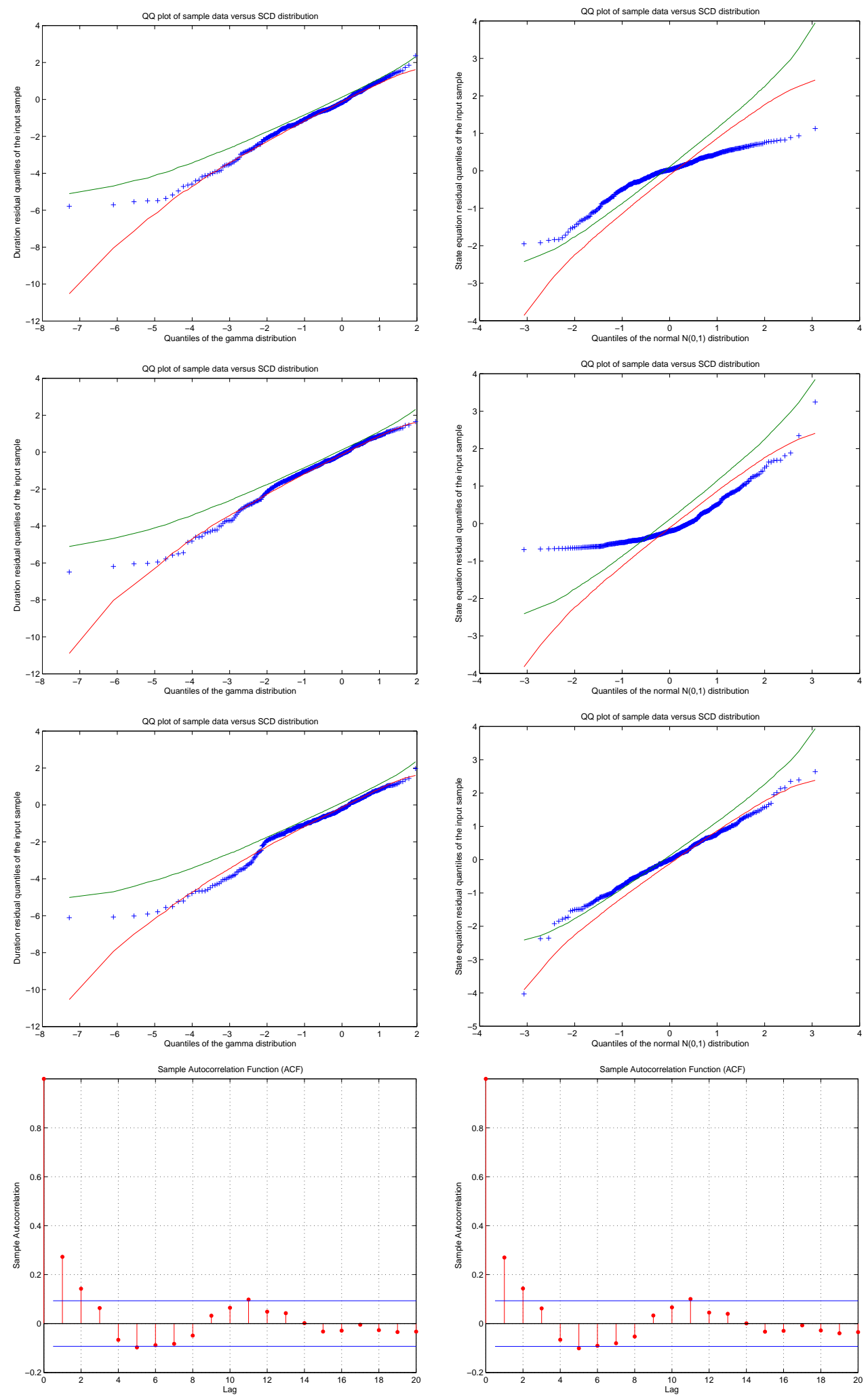

Figura H.24: De cima para baixo temos os gráficos de quantis dos resíduos obtidos com os filtros calculados em $\theta_{C E C F}$ com distribuição gama para a duração de preço de USD/EUR: (1) filtro de Kalman; (2) filtro Bayesiano; (3) filtro Gram-Charlier; (4) função de autocorrelação dos resíduos do filtro de Kalman.

À esquerda vemos os resíduos da equação de observação e à direita os resíduos da equação de estado. As linhas representam os limites do intervalo de $95 \%$ de confiança, obtidos através de simulação. 


\section{H.3.3 Ln duração de negócio}

Podemos observar pela figura H.17, que não conseguimos eliminar a sazonalidade dos dados com a transformação utilizada no capítulo 8, Assim vamos examinar o efeito de uma outra transformação nas durações de preço de USD/EUR. Para isso vamos utilizar a seguinte transformação:

$$
Y_{j}=\ln D_{j}
$$

onde $D_{i}$ é a duração. Podemos efetuar a dessazonalização na log-furação $Y_{j}$ por

$$
y_{j}=\frac{Y_{j}}{\phi\left(\tau_{j}\right)}
$$

onde a variável aleatória $y_{i}$ é modelada através do modelo SCD. Assim, podemos escrever

$$
D_{j}=e^{\phi\left(\tau_{j}\right) y_{j}} .
$$

Se chamarmos de a duração dessazonalizada de $D_{j}^{*}$, dada por

$$
D_{j}^{*}=e^{y_{j}}
$$

temos que

$$
\begin{aligned}
D_{j}^{*} & =e^{y_{j}} \\
& =e^{\frac{Y_{j}}{\phi\left(\tau_{j}\right)}} \\
& =e^{\frac{\ln D_{j}}{\phi\left(\tau_{j}\right)}} \\
& =e^{\ln D_{j}^{1 / \phi\left(\tau_{j}\right)}} \\
& =D_{j}^{1 / \phi\left(\tau_{j}\right)}
\end{aligned}
$$

Ou seja, neste caso, a dessazonalização é dada por

$$
D_{j}^{*}=D_{j}^{1 / \phi\left(\tau_{j}\right)}
$$

Para obter a função $\phi\left(\tau_{j}\right)$ utilizamos o estimador não paramétrico de Nadaraya-Watson, com largura de banda de 12960 (sugestão do EViews) com kernel Gaussiano. 

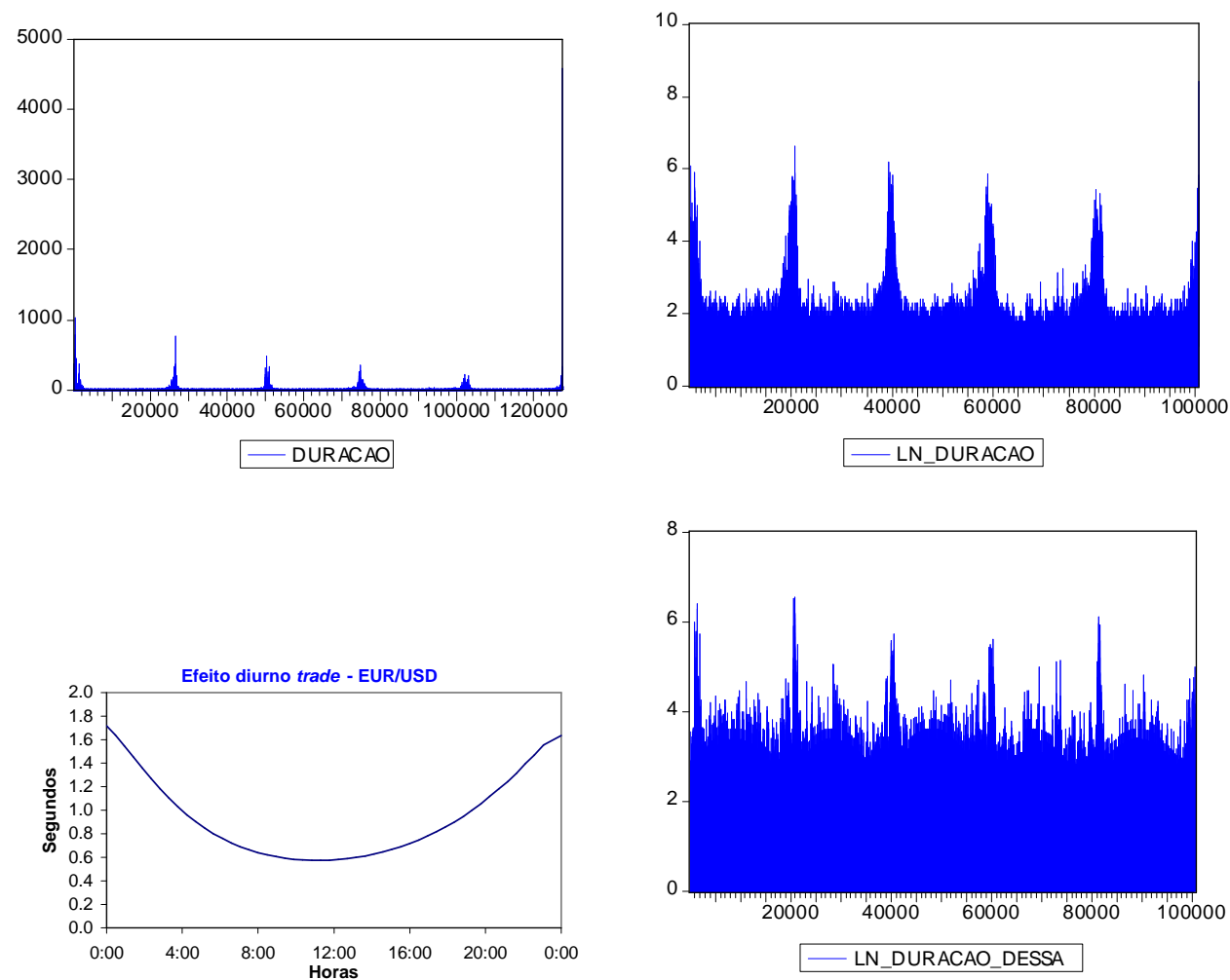

Figura H.25: Dados de USD/EUR:

Cima: duração de negócio (trade duration) e respectivo logaritmo.

Baixo: componente diurno do logaritmo da duração de negócio e logaritmo da duração de negócio dessazonalizado.

\begin{tabular}{|c|c|c|c|c|c|c|}
\hline \multirow{2}{*}{ Parâmetro } & \multicolumn{3}{|c|}{ Weibull } & \multicolumn{3}{|c|}{ Gamma } \\
\hline & QML & CECF & DECF & QML & CECF & DECF \\
\hline$\delta$ & 0.001 & 0.001 & 0.001 & 0.001 & 0.001 & 0.001 \\
\hline$\phi$ & 0.999 & 0.999 & 0.999 & 0.999 & 0.999 & 0.999 \\
\hline$\sigma \eta$ & 0.016 & 0.024 & 0.029 & 0.016 & 0.023 & 0.028 \\
\hline$\lambda$ & 1.271 & 1.327 & 1.308 & 1.407 & 1.572 & 1.629 \\
\hline Tempo (s) & 488.8 & 15.8 & 113.4 & 96.2 & 14.3 & 68.2 \\
\hline
\end{tabular}

Tabela H.7: Estimação dos parâmetros do modelo SCD para a duração de negócio (trade duration) de USD/EUR, considerando distribuições Weibull e gama para os erros. 

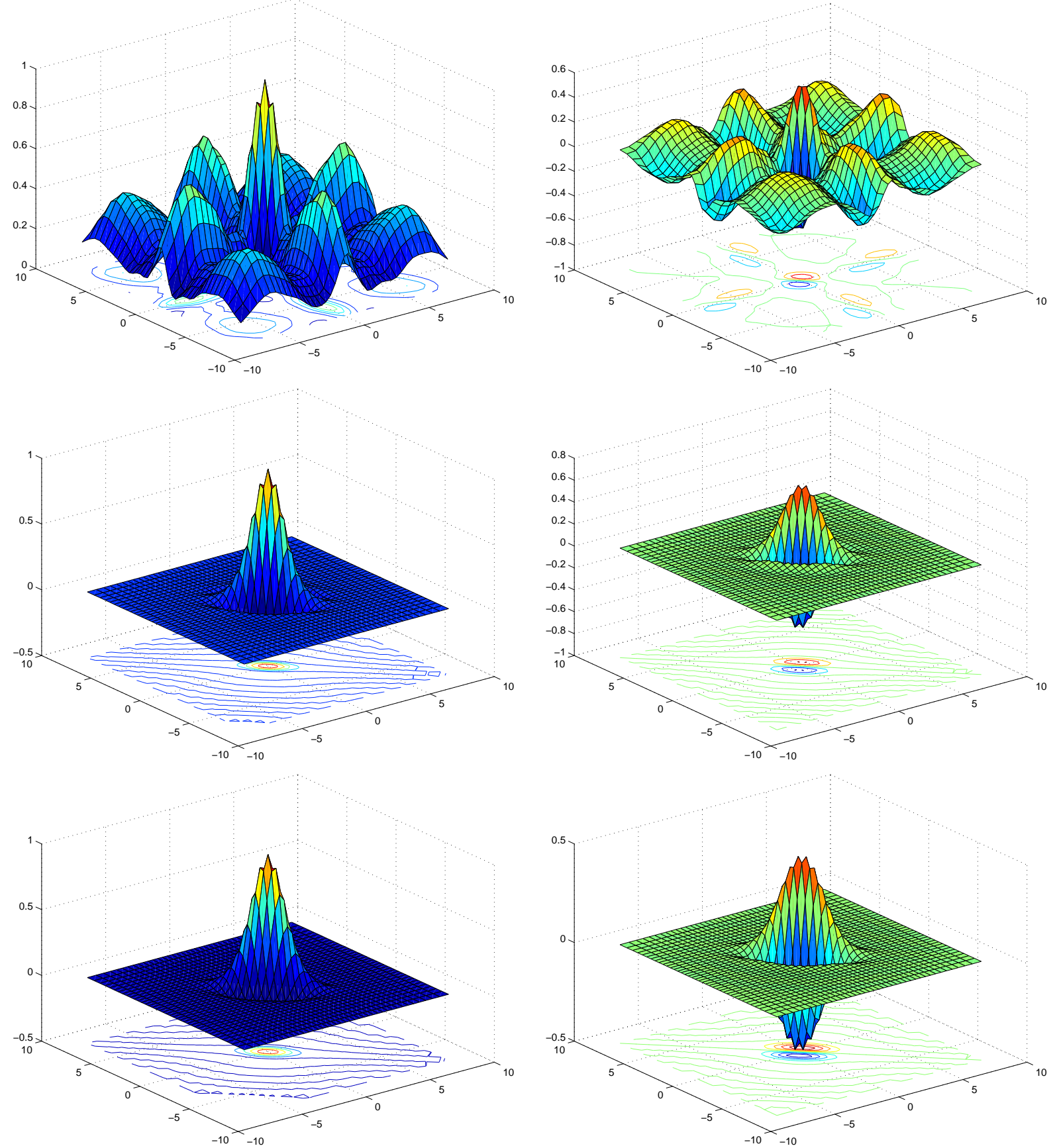

Figura H.26: Partes real (esquerda) e imaginária (direita) da função característica empírica (cima) e das funções características com distribuição gama para a duração de negócio (trade duration) de USD/EUR, calculadas em $\theta_{Q M L}$ (meio) e $\theta_{C E C F}$ (baixo), para a malha de 39 nós da quadratura de Gauss-Hermite. 

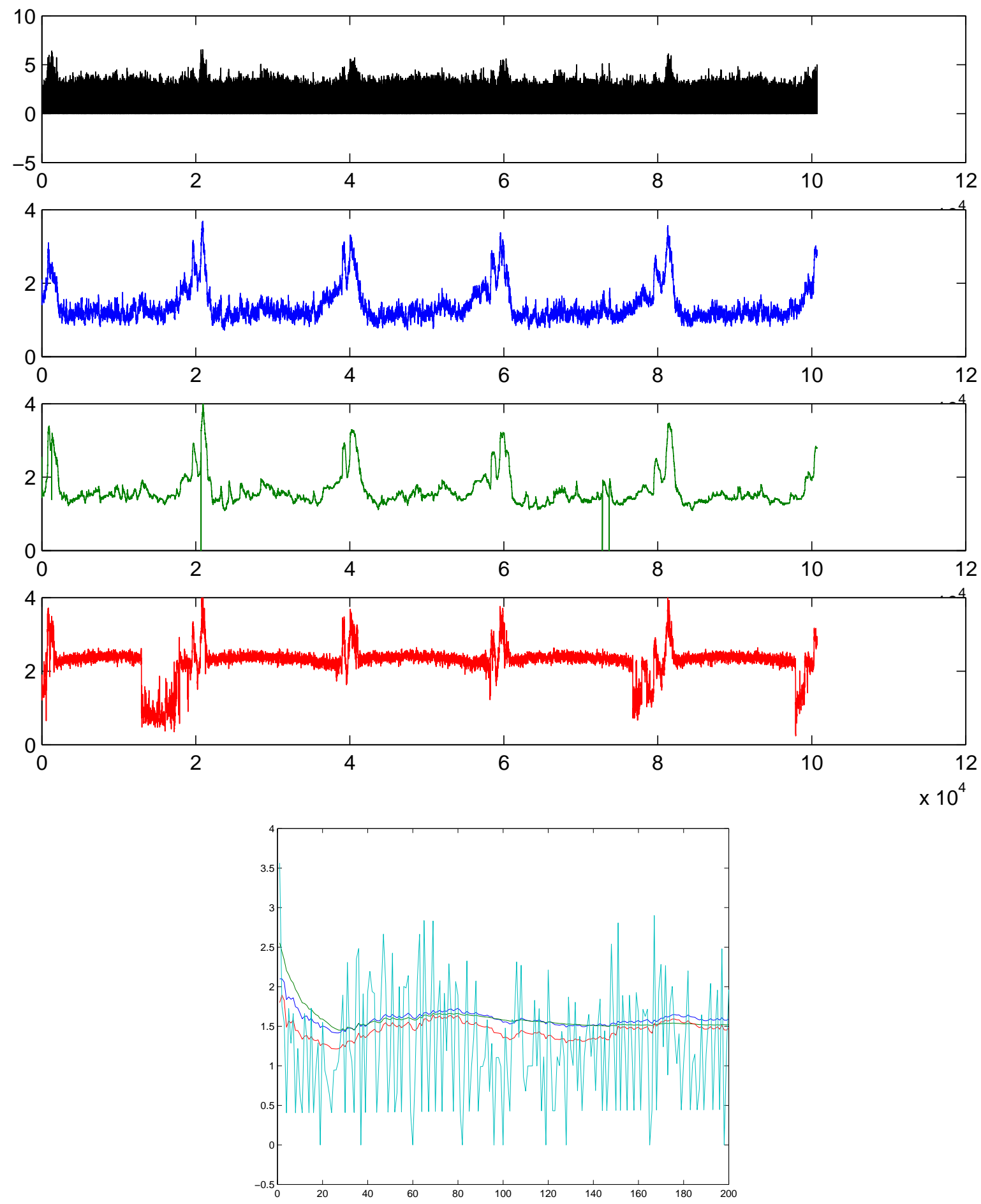

Figura H.27: Estimativa da variável latente para a duração de negócio (trade duration) de USD/EUR.

Cima: de cima para baixo temos os seguinte filtros calculados em $\theta_{C E C F}$ : (1) Log-durações; (2) filtro de Kalman; (3) filtro Bayesiano; (4) filtro Gram-Charlier.

Baixo: primeiras 200 observações dos filtros. 

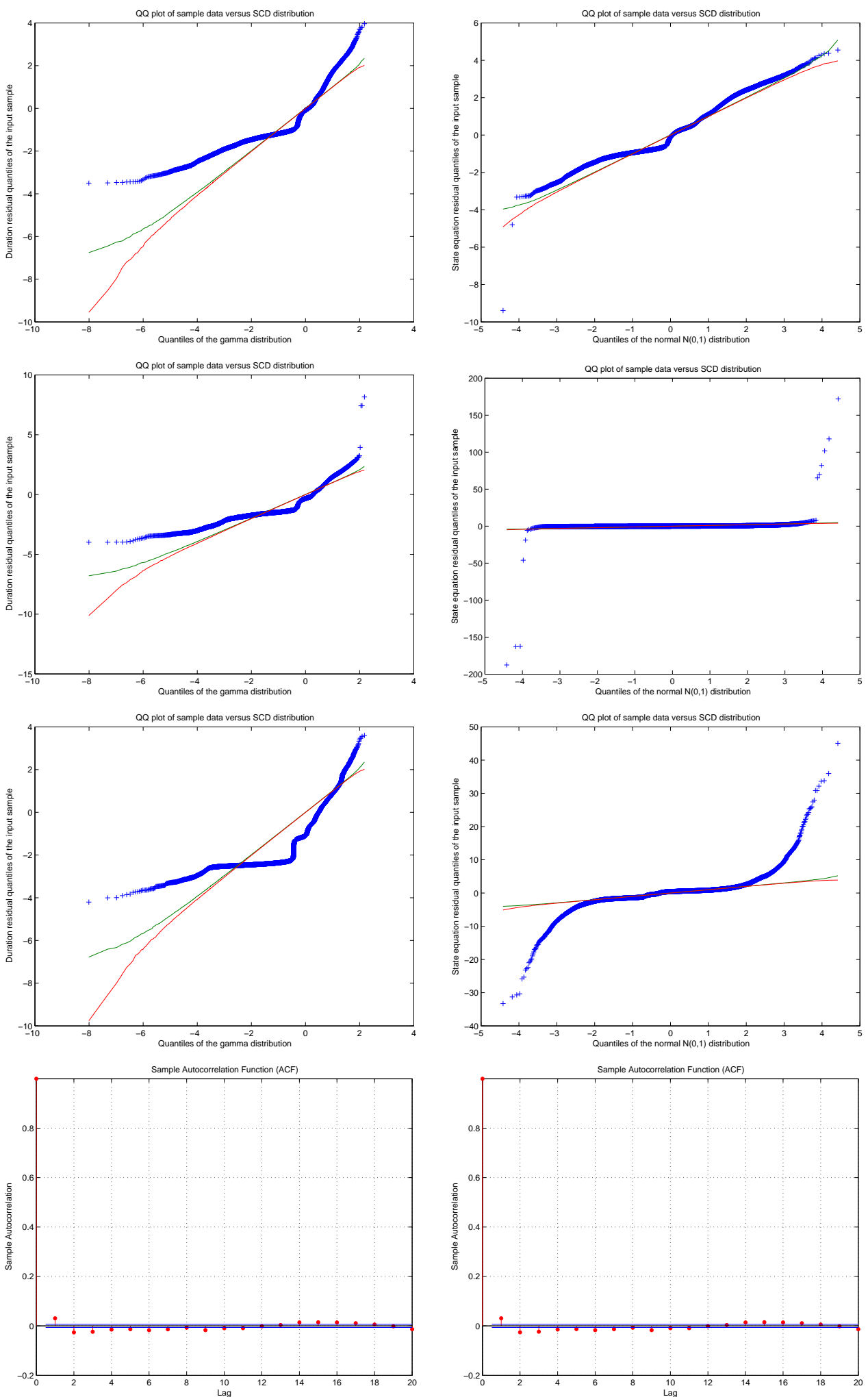

Figura H.28: De cima para baixo temos os gráficos de quantis dos resíduos obtidos com os filtros calculados em $\theta_{C E C F}$ com distribuição gama para a duração de negócio de USD/EUR: (1) filtro de Kalman; (2) filtro Bayesiano; (3) filtro Gram-Charlier; (4) função de autocorrelação dos resíduos do filtro de Kalman.

À esquerda vemos os resíduos da equação de observação e à direita os resíduos da equação de estado. As linhas representam os limites do intervalo de $95 \%$ de confiança, obtidos através de simulação. 



\section{Referências Bibliográficas}

Abramowitz, M. e Stegun, I. A. (1972). Handbook of Mathematical Functions with Formulas, Graphs and Mathematical Tables. Dover.

Amemiya, T. (1985). Advanced Econometrics. Harvard University Press.

Andrews, D. W. K. (1991). Heteroskedasticity and autocorrelation consistent covariance matrix estimation. Econometrica, 59(3):817-858.

Arad, R. W. (1980). Parameter estimation for symmetric stable distribution. International Economic Review, 21(1):209-220.

Balitskaya, E. O. e Zolotuhina, L. A. (1988). On the representation of a density by an Edgeworth series. Biometrika, 75(1):185-187.

Barton, D. E. e Dennis, K. E. (1952). The conditions under which Gram-Charlier and Edgeworth curves are positive and unimodal. Biometrika, 39(3/4):425-427.

Bauwens, L. e Veredas, D. (2004). The stochastic conditional duration model : A latent variable model for the analysis of financial durations. Journal of Econometrics, 119:381412.

Billingsley, P. (1995). Probability and Measure. John Wiley and Sons, third edição.

Bilodeau, M. e de Micheaux, P. L. (2005). A multivariate empirical characteristic function test of independence with normal margins. Journal of Multivariate Analysis, 95:345-369.

Bodie, Z.; Kane, A. e Marcus, A. J. (2002). Investments. McGraw-Hill/Irwin, fifth edição.

Briers, M.; Doucet, A. e Maskell, S. (2004). Smoothing algorithms for state-space models. Technical Report CUED/F-INFENG/TR.498, Signal Processing Group - Department of Engineering - University of Cambridge.

Brockwell, P. J. e Brown, B. M. (1981). High-efficiency estimation for the positive stable laws. Journal of the American Statistical Association, 76(375):626-631.

Brockwell, P. J. e Davis, R. A. (1991). Time Series: Theory and Methods. Springer, second edition edição. 
Brockwell, P. J. e Liu, J. (1992). Estimating the noise parameters from observations of a linear processes with stable innovations. Journal of Statistical Planning and Inference, $33: 175-186$.

Cabaña, A. e Quiroz, A. J. (2005). Using the empirical characteristic function in testing for the Weibull and the type I extreme value distributions. Test, 14(2):111-999.

Carrasco, M. e Florens, J.-P. (2000a). Estimation of a mixture via the empirical characteristic function. Working paper, http://www. econometricsociety.org/meetings/wc00/pdf/0514.pdf.

Carrasco, M. e Florens, J.-P. (2000b). Generalization of GMM to a continuum of moment conditions. Econometric Theory, 16:797-834.

Carrasco, M. e Florens, J.-P. (2002). Efficient GMM estimation using the empirical characteristic function. GREMAQ-University of Toulouse Working Paper, http://courses.ats.rochester.edu/carrasco/facweb/CarrascoPapers/chara8.pdf.

Chacko, G. e Viceira, L. M. (2003). Spectral GMM estimation of continuous-time processes. Journal of Econometrics, 116:259-292.

Challa, S.; Bar-Shalom, Y. e Krishnamurthy, V. (2000). Nonlinear filtering via generalized edgeworth series and gauss hermite quadrature. IEEE Transactions on Signal Processing, 48(6):1816-1820.

Chen, A. e Bickel, P. (2003). Efficient Independent Component Analysis (i). TECHNICAL REPORTS nº34, Statistics Department, University of California, Berkeley.

Csörgo, S. (1981). Limit behavior of the empirical characteristic function. The Annals of Statistics, 9(1):130-144.

Csörgo, S. e Heathcote, C. R. (1987). Testing for symmetry. Biometrika, 74(1):177-184.

Davidson, J. (1994). Stochastic Limit Theory. Oxford University Press.

der Vaart, A. W. V. (1998). Asymptotic Statistics. Cambridge University Press.

Draper, N. R. e Tierney, D. (1972). Regions of positive and unimodal series expansion of the Edgeworth and Gram-Charlier approximations. Biometrika, 59(2):463-465.

Duffie, D.; Pan, J. e Singleton, K. (2000). Transform analysis and asset pricing for affine jump-diffusions. Econometrica, 68(6):1343-1376.

Durbin, J. e Koopman, S. J. (2000). Time series analysis of non-gaussian observations based on state space models from both classical and bayesian perspectives. Journal of the Royal Statistical Society, 62:3-56. 
Engle, R. F. e Russell, J. R. (1998). Autoregressive conditional duration: A new model for irregularly spaced transaction data. Econometrica, 66(5):1127-1162.

Epps, T. W. (1987). Testing that a stationary time series is gaussian. The Annals of Statistics, 15(4):1683-1698.

Epps, T. W. e Pulley, L. B. (1983). A test for normality based on the empirical characteristic function. Biometrika, 70(3):723-726.

Fearnhead, P. (1998). Sequential Monte Carlo Methods in Filter Theory. PhD thesis, Merton College, University of Oxford.

Feuerverger, A. (1990). An efficiency result for the empirical characteristic function in stationary time-series models. The Canadian Journal of Statistics, 18(2):155-161.

Feuerverger, A. e McDunnough, P. (1981a). On some Fourier methods for inference. Journal of the American Statistical Association, 76(374):379-387. Theory and Methods Section.

Feuerverger, A. e McDunnough, P. (1981b). On the efficiency of empirical characteristic function procedures. Journal of the Royal Statistics Society - Series B, 43(1):20-27.

Feuerverger, A. e McDunnough, P. (1984). On statistical transform methods and their efficiency. The Canadian Journal of Statistics, 12(4):303-317.

Feuerverger, A. e Mureika, R. A. (1977). The empirical characteristic function and its applications. The Annals of Statistics, 5(1):88-97.

Frangakis, C. F. (2005). Lecture notes on advanced statistical theory I-II. Course 140.771-2, Dep. of Biostatistics, Johns Hopkins School of Public Health.

Gupta, R. D. e Kundu, D. (2000). Generalized exponential distributions. Australian and New Zealand Journal of Statistics, 41(16):173-188.

Hansen, L. P. (1982). Large sample properties of generalized method of moments estimators. Econometrica, 50(4):1029-1054.

Hayashi, F. (2000). Econometrics. Princeton University Press.

Heathcote, C. R. (1977). The integrated squared error estimation of parameters. Biometrika, $64(2): 255-264$.

Hesse, C. H. (1990). Rates of convergence for the empirical distribution and the empirical characteristic function of a broad class of linear processes. Journal of Multivariate Analysis, $35: 186-202$.

Hong, Y. (1999). Hypothesis testing in time series via the empirical characteristic function: A generalized spectral density approach. Journal of the American Statistical Association, 94(448):1201-1220. 
Hong, Y. (2000). Generalized spectral tests for serial dependence. Journal of the Royal Statistical Society - Series B, 62(3):557-574.

Hu, Y. e Ridder, G. (2006). Estimation of nonlinear models with mismeasured regressors using marginal information. Working paper, http://www.eco.utexas.edu/faculty/Hu/EIV-marg-final.pdf.

Huber, P. J. (1981). Robust Statistics. John Wiley and Sons.

Ibragimov, I. A. e Linnik, Y. V. (1971). Independent and Stationary Sequences of Random Variables. Wolters-Noordhoff Publishing Groningen.

Jäckel, P. (2005). A note on multivariate Gauss-Hermite quadrature.

Jennrich, R. I. (1969). Asymptotic properties of non-linear least squares estimators. The Annals of Mathematical Statistics, 40:633-643.

Jiang, G. J. e Knight, J. L. (2001). Efficient estimation of Markov models where the density is unknown. Working paper, http://www.vanderbilt.edu/econ/sempapers/Knight.pdf.

Jiang, G. J. e Knight, J. L. (2002). Estimation of continuous time processes via the empirical characteristic function. Journal of Business and Economic Statistics, 20:198-212.

Jondeau, E. e Rockinger, M. (1999). Estimating Gram-Charlier expansions with positivity constraints. Technical report, Banque de France, Notes d'Études et de Recherche, NER 56.

Kellermeier, J. (1980). The empirical characteristic function and large sample hypothesis testing. Journal of Multivariate Analysis, 10:78-87.

Kitagawa, G. (1987). Non-gaussian state-space modelling of nonstationary time series. Journal of the American Statistical Association, 82(400):1032-1063.

Klar, B. e Meintanis, S. G. (2005). Tests for normal mixtures based on the empirical characteristic function. Computational Statistics and Data Analysis, 49:227-242.

Kleiber, C. e Kotz, S. (2003). Statistical Size Distributions in Economics and Actuarial Science. Wiley Interscience.

Knight, J. L. e Satchell, S. E. (1997). The cumulant generating function estimation method. Econometric Theory, 13:170-184.

Knight, J. L.; Satchell, S. E. e Yu, J. (2002). Estimation of the stochastic volatility model by the empirical characteristic function. Australian New Zealand Journal of Statistics, $44(3): 319-335$.

Knight, J. L. e Yu, J. (2002). Empirical characteristic function in time series estimation. Econometric Theory, 18:691-721. 
Kogon, S. M. e Williams, D. B. (1998). Characteristic Function Based Estimation of Stable Distribution Parameters, pp. 311-335. Birkhäuser.

Koutrouvelis, I. A. (1982). Estimation of location and scale in Cauchy distributions using the empirical characteristic function. Biometrika, 69(1):205-213.

Kreyszig, E. (1978). Introductory Functional Analysis with Applications. John Wiley and Sons.

Lacey, M. T. (1989). Laws of the iterated logarithm for the empirical characteristic function. The Annals of Probability, 17(1):292-300.

Leitch, R. A. e Paulson, A. S. (1975.). Estimation of stable law parameters: Stock price behavior application. Journal of the American Statistical Association, 70(351):690-697.

Li, T. e Hsiao, C. (2004). Robust estimation of generalized linear models with measurement errors. Journal of Econometrics, 118:51-65.

Lima, E. L. (2002). Curso de Análise, volume 1. IMPA - Instituto de Matemática Pura e Aplicada, $10^{\mathrm{a}}$ edição.

Lukacs, E. (1970). Characteristic Functions. Griffin, London, 2nd. edição.

Lukacs, E. (1983). Developments in Characteristic Functions. Griffin, London.

Lütkepohl, H. (2005). New Introduction to Multiple Time Series Analysis. Springer.

Madhavan, A. (2000). Market microstructure: A survey. Journal of Financial Markets, $3: 205-258$.

Marcus, M. (1981). Weak convergence of the empirical characteristic function. The Annals of Probability, 9(2):194-201.

Meintanis, S. e Ushakov, N. G. (2004). Binned goodness-of-fit tests based on the empirical characteristic function. Statistics and Probability Letters, 69:305-314.

Meintanis, S. G. (2005). Consistent tests for symmetric stability with finite mean based on the empirical characteristic function. Journal of Statistical Planning and Inference, 128:373-380.

Montfort, K. V. e Mooijaart, A. (1989). Estimation of regression coefficients with the help of characteristic functions. Journal of Econometrics, 41:267-278.

Murata, N. (2001). Properties of the empirical characteristic function and its application to testing for independence. In Proceedings of 3rd International Conference on ICA and Blind Source Separation, pp. 19-24, San Diego, CA.

Murota, K. e Takeuchi, K. (1981). The studentized empirical characteristic function and its application to test for the shape of distribution. Biometrika, 68(1):55-65. 
Nakamura, M.; Pérez, F. J. e Quiroz, A. J. (1996). Estimation of multivariate Box-Cox transformation to elliptical symmetry via the empirical characteristic function. Annals of the Institute of Statistical Mathematics, 48(4):687-709.

Newey, W. K. e West, K. D. (1994). Automatic lag selection in covariance matrix estimation. Review of Economic Studies, 61:631-653.

Pacurar, M. (2006). Autoregressive conditional duration (ACD) models in finance: A survey of the theoretical and empirical literature. Working paper, http://neumann.hec.ca/gestiondesrisques/06-10.pdf.

Parzen, E. (1962). On estimation of a probability density function and mode. The Annals of Mathematical Statistics, 33:1065-1076.

Paulson, A. S. e Delehanty, T. A. (1985). Modified weighted squared error estimation procedures with special emphasis on the stable laws. Communications in Partial Differential Equations, 14(4):927-972.

Paulson, A. S.; Holcomb, E. W. e Leitch, R. A. (1975). The estimation of the parameters of stable laws. Biometrika, 62(1):163-170.

Press, S. J. (1972). Estimation in univariate and multivariate stable distributions. Journal of the American Statistical Association, 67(340):842-846.

Press, W. H.; Flannery, B. P.; Teukolsky, S. A. e Vetterling, W. T. (1997). Numerical Recipes in C. Cambridge University Press.

Robert, C. P. e Casella, G. (2004). Monte Carlo Statistical Methods. Springer, 2nd. edição.

Rockinger, M. e Semenova, M. (2005). Estimation of jump-diffusion processes via empirical characteristic function. Technical Report 150, FAME - International Center for Financial Asset Management and Engineering, Université de Genève. http://www.fame.ch/library/EN/RP150.pdf.

Rubin, H. (1956). Uniform convergence of random functions with applications to statistics. Annals of Mathematical Statistics, 27:200-203.

Schmidt, P. (1982). An improved version of the Quandt-Ramsey MGF estimator for mixtures of normal distributions and switching regressions. Econometrica, 50(2):501-516.

Serfling, R. J. (1980). Approximation Theorems of Mathematical Statistics. John Wiley and Sons.

Shiryaev, A. N. (1991). Probability. Springer, 2nd. edição.

Shumway, R. H. e Stoffer, D. S. (2000). Time Series Analysis and its Applications. Springer. 
Silva, A. C.; Prange, R. E. e Yakovenko, V. M. (2004). Exponential distribution of financial returns at mesoscopic time lags: A new stilized fact. Physica A, pp. 227-235.

Singleton, K. J. (2001). Estimation of affine asset pricing models using the empirical characteristic function. Journal of Econometrics, 102:111-141.

Singleton, K. J. (2006). Empirical Dynamic Asset Pricing: Model Specification and Econometric Assessment. Princeton University Press.

Smith, P. J. (1995). A recursive formulation of the old problem of obtaining moments from cumulants and vice-versa. The American Statistician, 49(2):217-218.

Sorenson, H. W. (1988). Recursive Estimation for Nonlinear Dynamic Systems, chapter 6, pp. 127-164. CRC.

Sorenson, H. W. e Stubberud, A. R. (1968). Non-linear filtering by approximation of the a posteriori density. International Journal of Control, 8(1):33-51.

Srinivasan, K. (1970). State estimation by orthogonal expansion of probability distributions. IEEE Transactions on Automation Control, AC-15(1):3-10.

Strickland, C. M.; Forbes, C. S. e Martin, G. M. (2005). Bayesian analysis of the stochastic conditional duration model. Working paper, http://www-personal.buseco.monash.edu.au/ gmartin/stricklandetal_SCD_2005.pdf.

Strickland, C. M.; Forbes, C. S. e Martin, G. M. (2006). Bayesian analysis of the stochastic conditional duration model. Computational Statistics and Data Analysis, 50:2247-2267.

Stuart, A. e Ord, J. K. (1994). Kendall's Advanced Theory of Statistics, volume 1 - Distribution Theory. Arnold, sixth edição.

Stute, W. (1983). Empirical processes indexed by smooth functions. Stochastic Processes and their applications, 14:55-66.

Thorton, J. C. e Paulson, A. S. (1977). Asymptotic distribution of characteristic functionbased estimators for the stable laws. Sankhya: The Indian Journal of Statistics, 39:341-354.

Tsay, R. S. (2002). Analysis of Financial Time Series. John Wiley and Sons.

Wells, M. T. (1992). The weak approximation of the empirical characteristic function process when parameters are estimated. Stochastic Processes and their applications, 40:83-102.

White, H. (2000). Asymptotic Theory for Econometricians. Academic Press, revised edição.

Yan, B. e Zivot, E. (2003). Analysis of high-frequency financial data with s-plus. Department of Economics, University of Washington.

Yu, J. (2004). Empirical characteristic function estimation and its application. Econometric Reviews, 23(2):93-123. 
Yukich, J. E. (1985). Weak convergence of the empirical characteristic function. Transactions of the American Mathematical Society, 95(3):470-473. 